\title{
The Allocation of Attentional Resources across the Visual Field: Impact of Eccentricity and Perceptual Load
}

\author{
D I S S E R T A T I O N
}

zur Erlangung des mathematisch-naturwissenschaftlichen Doktorgrades

"Doctor rerum naturalium"

der Georg-August-Universität Göttingen

vorgelegt von

Mag. Carmen Morawetz

aus

Kirchdorf an der Krems 
Referent: Prof. Dr. Stefan Treue

Korreferent: Dr. Peter Dechent

Weitere Referentin: Prof. Dr. Nicole von Steinbüchel

Tag der mündlichen Prüfung: 7. Juli 2008 


\section{Acknowledgements}

„Es liegt ein tiefes und gründliches Glück darin, dass die Wissenschaft Dinge ermittelt, die Stand halten und immer wieder den Grund zu neuen Ermittlungen abgeben: es könnte ja anders sein!“

Friedrich Nietzsche

An dieser Stelle möchte ich gerne all jenen Menschen danken, die mich während meines Doktorats begleitet und unterstützt haben.

$\mathrm{Zu}$ allererst bedanke ich mich ganz herzlich bei meiner Familie, die alle Höhen und Tiefen der letzten Jahre mit mir geteilt hat, mir stets mit Rat zur Seite stand und mir Kraft gegeben hat.

Ein großes Dankeschön geht auch an meine Betreuer, Dr. Peter Dechent, Prof. Dr. Stefan Treue und Prof. Dr. Nicole von Steinbüchel für ihre dauerhafte Unterstützung, ihr Interesse an der vorliegenden Arbeit, sowie die gute Betreuung und Anleitung.

Weiterer Dank gebührt meinen Arbeitskollegen in der MR-Forschungsgruppe, ohne die diese Arbeit nicht möglich gewesen wäre: Jürgen Baudewig, Petra Holz und Ilona Pfahlert.

Abschließend möchte ich mich noch beim Europäischen Graduiertenkolleg "From Neuroplasticity to Molecules to Systems (GRK 632)" für die finanzielle Unterstützung und Förderung bedanken. 


\section{Contents}

List of abbreviations___ 7

I Introduction

II Functional magnetic resonance imaging __ 11

2.1 Imaging System 12

2.2 Physical principles _ 12

2.3 Physiological principles

2.4 Data analyses___ 20

2.4.1 Analysis of anatomical data___ 22

2.4.2 Analysis of functional data __ 24

2.5 General MR parameters __ 28

III Selective visual attention __ 29

3.1 Organization of visual cortex

3.2 Space-based theories of attention

3.2.1 Single spotlight of attention___ 39

3.2.2 Zoom lens model __ 44

3.2.3 Multiple spotlights of attention __ 47

IV Split of attentional resources in human visual cortex___ 53

4.1 Introduction

4.2 Methods

4.2.1 Subjects 55

4.2.2 Attention Tasks _ 56

4.2.3 Retinotopic Mapping __ 59

4.2.4 Magnetic Resonance Imaging and Data Analysis___ 62 
4.3 Results 63

4.3.1 Behavioral Results 63

4.3.2 Neuroimaging Results 64

4.3.2.1 Experiment 1 64

4.2.3.2 Experiment 2 65

4.2.3.3 Data Reproducibility 68

4.4 Discussion 68

$V$ Interaction between emotion and attention 72

5.1 Anatomical and functional correlates of emotion recognition in faces 77

5.1.2 Visual cortices 79

5.1.2 Amygdala 84

5.1.3 Other brain regions 90

5.1.3.1 Prefrontal cortex 90

5.1.3.2 Anterior cingulate cortex 93

5.1.3.3 Somatosensory-related cortices 95

5.1.3.4 Insular cortex and basal ganglia 96

5.2 Processing of emotional stimuli 98

5.2.1 Automatic processing 98

5.2.2 Controlled processing 103

5.2.3 Neuromodulatory factors 107

5.2.3.1 Interaction between attentional load and location of affective stimuli 108

5.2.3.2 Spatial frequency of affective stimuli 109

5.2.3.3 Stimulus salience 111

VI Face processing in the human amygdala depending on attentional load and spatial location 
6.2.3 Experimental procedure

6.2.4 Image processing and analysis

6.3 Results 121

6.3.1 Behavioural Results 121

6.3.2 Neuroimaging Results 122

6.3.2.1 Main task effects 122

6.3.2.2 Attend-faces versus ignore-faces conditions 126

6.3.2.3 Amygdala region of interest analysis 128

6.3.2.4 Fusiform gyrus region of interest analysis 129

6.4 Discussion 131

6.4.1 Amygdala 131

6.4.2 Fusiform gyrus 134

6.4.3 Whole-brain analysis 135

6.5 Conclusion 136

VII Conclusion \& Outlook 137

VIII References 141 


\section{List of abbreviations}

2D Two-dimensional

3D Three-dimensional

AC Anterior commissure

ACC Anterior cingulate cortex

ACS Attentional Control Scale

BA Brodmann Area

BOLD Blood oxygenation level dependent

CBF Cerebral blood flow

CBV Cerebral blood volume

dACC Dorsal anterior cingulated cortex

DLPFC Dorsolateral prefrontal cortex

EPI Echo planar imaging

ERP Event-related potentials

FDR False discovery rate

FEF Frontal eye fields

FFA Fusiform face area

FFX Fixed-effects analysis

fMRI Functional magnetic resonance imaging

FOV Field of view

FT Fourier transform

FWHM Full-width-half-maximum

GLM General linear model

HRF Haemodynamic response function

IT Inferotemporal cortex

LGN Lateral geniculate nucleus

M Magnocellular

MEG Magnetoencephalography

MRI Magnetic resonance imaging

NMR Nuclear magnetic resonance

OFC Orbitofrontal cortex

P Parvocellular 
PANAS Positive and Negative Affective Schedule

PC Posterior commissure

PET Positron-emission tomography

PFC Prefrontal cortex

PPA Parahippocampal place area

PPC Posterior parietal cortex

RF Radiofrequency

RFX Random-effects analysis

ROI Region of interest

ROI Region-of-interest

RSVP Rapid serial visual presentation

RT Reaction time

SAM Self Assessment Manikin

SNR Signal-to-noise ratio

SPM Statistical parametric mapping

SSVP Steady-state visual evoked potential

STS Superior temporal sulcus

TOS Transverse occipital sulcus

VMPFC Ventromedial prefrontal cortex 


\section{Introduction}

"Feelings are much like waves, we can't stop them from coming but we can choose which one to surf."

Jonatan Mårtensson

Emotions colour our everyday life in many ways, give meaning or purpose to actions and experiences. For emotions not only colour human behavior but to a large extent guide it as well. In order to understand such guidance, it is necessary to consider the interaction between attention and emotion. Attention as an information-selection process gives priority to behaviorally relevant stimuli. One of the principal indicators of the importance of these stimuli is the emotional content. The issue whether incoming emotional stimuli are processed preattentively or whether they preferentially engage mechanisms of selective attention was investigated in the course of this thesis in several experiments using functional magnetic resonance imaging (fMRI).

As both studies presented in this thesis are based upon fMRI, in chapter 2 the physical and physiological principles of fMRI are introduced, followed by a brief description of the data analyses.

Chapter 3 illustrates the concept of selective visual attention focusing in the first part on the organization of the visual cortex and in the second part on metaphors describing the allocation of attention in the visual field. Of particular interest are spotlight-theories claiming that visual attention acts like a spotlight illuminating everything covered by its beam (single spotlight theory, zoom lens model, multiple spotlight theory). Recent findings give rise to the assumption that the spotlight of attention can be split. Therefore, the neuronal basis underlying the split of selective attention among spatially separated locations in the visual field was investigated in the first study.

In chapter 4 the first fMRI study entitled 'Split of Attentional Resources in Human Visual Cortex' is presented. The aim of the study was the verification of the mutliple spotlight theory and its extension with regard to distant parts of the visual field. The representation 
of multiple retinotopically specific regions of activation in striate and extrastriate visual cortex indicated a split of attentional resources.

In the second study, I aimed to examine the allocation of attentional resources during attentionally demanding tasks involving emotional stimuli. The cognitive control of emotion plays a crucial role in human adaptive behaviour depending on interactions between prefrontal and cingulate control systems and cortical and subcortical emotiongenerative systems. In the first part of chapter 5 the most relevant regions implicated in the processing of emotions, especially in the processing of emotional facial expressions, are described. A special emphasis is placed on the role of the amygdala as the key structure within the emotion processing system. In the second part, the processing of emotional facial expressions itself is outlined. On the basis of neuroimaging results two different views emerged supporting either the automatic or controlled view of processing emotionladen stimuli. Because of different patterns of imaging results the issue remained unresolved. Several neuromodulatory factors such as spatial location of the emotional stimuli, spatial frequency and attentional load are discussed in order to disentangle the discrepancies.

Capter 6 provides the findings of the second fMRI study 'Face processing in the human amygdala depending on attentional load and spatial location in the visual field'. In order to characterize the relationship between the direction of attention and the processing of emotional faces, I investigated the underlying mechanisms of face processing in dependence of different attentional load conditions and spatial position of the emotional stimuli in the visual field. Attention was either directed to images displaying facial expressions or to non-emotional stimuli. The results support the view of a controlled processing of emotional stimuli which is dependent upon the availability of the attentional resources.

Finally, a general conclusion summarizes the obtained results and the outlook deals with open unresolved questions thereby introducing topics for future studies. 


\section{Functional magnetic resonance imaging}

„BOLD-contrast imaging (..). quickly became a mainstay of cognitive neuroscience. It is an accessible option for measuring brain activity with relatively high spatial resolution (...). From the detailed characterization of the function of human visual brain areas to the discovery of areas that are potentially involved in higher cognitive functions, such as face recognition, empathy and self-awareness, the possibilities revealed by fMRI seem endless.”

I-han Chou, Senior Editor, Nature

Nuclear magnetic resonance (NMR) has a long history dating back to the 1940s, when Felix Bloch (1946) and Edward Purcell and colleagues (1946) simultaneously detected a resonance phenomenon in samples placed in a magnetic field. Imaging using NMR was first demonstrated in the 1970s by Paul Lauterbur (1973), and has since seen huge application in diagnostic radiology. Whilst NMR is a nuclear effect, in the sense that it is the constituents of the atomic nucleus that resonate, NMR does not involve radioactivity. Indeed, the term 'nuclear' has been dropped in medical circles to avoid patient concern, yielding the now familiar appellation 'magnetic resonance imaging' (MRI). Initially MRI provided superb anatomical information, and inherent in the data was important metabolic and physiological information. An opening for MRI in the area of functional brain imaging emerged when it was discovered that during changes in neuronal activity there are local changes in the amount of oxygen in the tissue (Fox \& Raichle, 1986; Fox et al., 1988). By combining this observation with a much earlier finding by Pauling and Coryell (1936) that changing the amount of oxygen carried by haemoglobin changes the degree to which haemoglobin disturbs a magnetic field, Ogawa and colleagues (1990) were able to demonstrate that in vivo changes in blood oxygenation could be detected with MRI. The MRI signal arising from this unique combination of brain physiology (Fox \& Raichle, 1986) and NMR physics (Pauling \& Coryell, 1936; Thulborn et al., 1982) became known as the blood oxygenation level dependent (BOLD) signal (Ogawa et al., 1990). Several 
demonstrations of BOLD signal changes in normal humans during functional brain activation followed (Ogawa et al., 1992; Kwong et al., 1992; Bandettini et al., 1992; Frahm et al., 1992), which gave birth to the rapidly developing field of functional MRI (fMRI).

Using MRI it is possible to depict anatomical as well as functional aspects of the human body in a three-dimensional (3D) way. The following sections give a brief overview of the physical and physiological basic principles underlying fMRI.

\section{$\underline{2.1}$ Imaging System}

The MRI system consists of three main components: magnet, computer, and operating system. The static magnetic field is the core component within the imaging system. Solenoidal magnets capable of achieving high field strengths and having a bore large enough to accommodate human subjects are invariably constructed with superconducting technology in order to achieve sufficient currents. The strength of the magnetic field most commonly used in neuroscience is 3 Tesla, which is 60.000 times stronger than the magnetic field of the earth. The subject aperture is usually approximately $60 \mathrm{~cm}$ in diameter. The head of the subject is placed in a head coil (radiofrequency (RF) coils), which is of great importance to generate and receive electromagntic fields at the resonant frequency of the atomic nuclei within the static magnetic field. The scanner is controlled by a computer control system installed in a separate room. For the presentation of experimental tasks a separate computer is used, which projects the stimuli onto LCD googles in the scanner room. This computer is further connected to response devices in order to collect the behavioral data related to the tasks.

\section{$\underline{2.2 \text { Physical principles }}$}

The basic physical principles are valid for both anatomical as well as functional MRI. The operational principles of MRI can be subdivided into two main components: the excitation and recording of electromagnetic signals and the construction of 3D images.

MRI takes advantage of hydrogen nuclei in the human body, which behave like tiny magnets. Hydrogen makes up $80 \%$ of all atoms found in the human body as part of water $\left(\mathrm{H}_{2} \mathrm{O}\right)$ and lipids and because of its abundance, hydrogen is the most commonly imaged nucleus in MRI.

As the hydrogen nucleus consists of a single proton, thermal energy causes the proton to spin about itself. This spin motion has two effects. First, because the proton has an odd- 
numbered atomic mass (i.e., 1), its spin results in a property called angular momentum, which has both magnitude and direction. Second, because the proton carries a positive charge, its spin generates an electrical current. This loop current induces a torque when it is placed within a magnetic field, which is called the magnetic moment (Figure 1). Quantum mechanics dictates that a spin can have different energies depending on the orientation of its magnetic moment with respect to the applied magnetic field: when the magnetic moment is aligned with the field, its energy will be lower than when it opposes the field. For the simple spin system hydrogen-1, the magnetic moment can therefore have two orientations, either against/anti-parallel to the magnetic field (high energy state) or along/parallel to it (low energy state). All of the signals generated in MRI are based on small differences between these two energy states.

If both magnetic and angular momentum are present in a nucleus, it possesses the NMR property. Only nuclei with an odd atomic mass number and an odd charge number have a spin and can be studied using magnetic resonance. Indeed, hydrogen-1 has one of the strongest of all nuclear moments, and therefore is an excellent candidate for biomedical imaging.

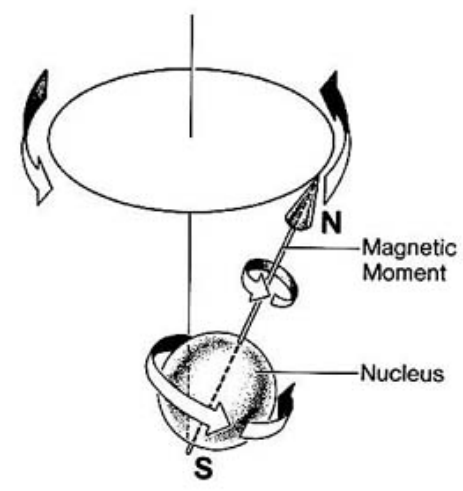

\section{Figure 1}

A fundamental physical property of protons and neutrons is that they possess an angular momentum, which is often referred to as a 'spin'. Atomic nuclei create magnetic fields like those of microscopic current loops. The strength of a nucleus' intrinsic magnetism is called its magnetic moment. When atomic nuclei are placed in a magnetic field, they resemble tiny bar magnets with north and south poles. The direction of its magnetic moment precesses about the direction of the field with a frequency proportional to the strength of the external field. Magnetic resonance occurs when the strength of the external magnetic field is manipulated until the precession frequency of the particle matches that of an applied RF. This causes the particle's magnetic moment to change orientation relative to the external magnetic field (from www.beyonddiscovery.org). 
In the absence of any strong magnetic field, the nuclear magnetic moments of the hydrogen protons are oriented in random directions (Figure 2a). The sum of all magnetic moments from spins of different orientations is called net magnetization (M), which is infinitesimally small under normal conditions. When the net magnetization is at equilibrium no signal is detected in a conducting coil placed around the sample (since there is no time-dependent change in the net magnetization). To increase the magnetization of the protons, a strong magnetic field must be applied. In a magnetic field $\left(\mathrm{B}_{0}\right)$ protons change their orientation and tend to align along the direction of the magnetic field, defined as $\mathrm{z}$ direction. Slightly more spins will align parallel to the field than anti-parallel to the field (Figure 2b), resulting in a net magnetization from the ensemble.
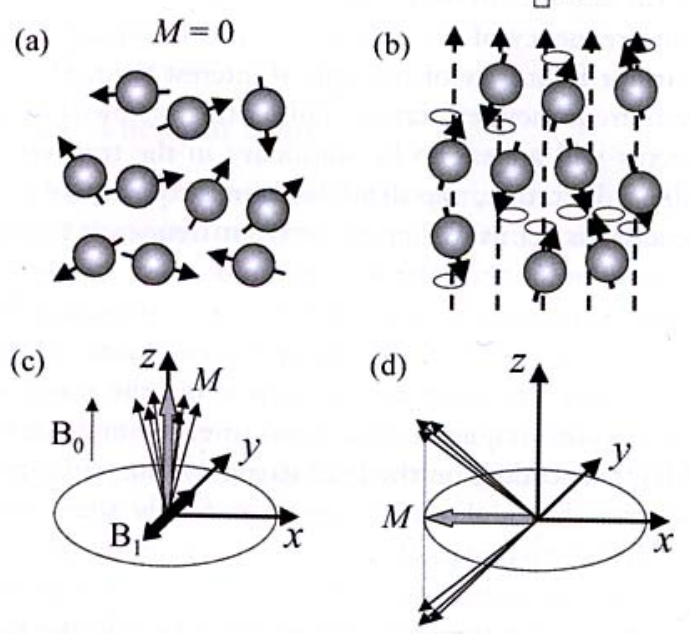

\section{Figure 2}

Behaviour of a sample when placed in a strong magnetic field $\left(\mathrm{B}_{0}\right)$. (a) The nuclear magnetic moments are initially randomly oriented. (b) Gradually the moments align either with the field or against it. The slight preferential alignment along the direction of the field acts like a single magnetization vector $M$. (c) An oscillating $B_{1}$ magnetic field can change the orientation of some of the nuclear moments until there is a net magnetization vector in the xy plane (d) (from Jezzard \& Clare, 2001).

The difference between the parallel and anti-parallel states leads to an important equation relating the magnetic field strength to the expected resonance frequency of the spins. If the sample is excited by an additional RF pulse magnetic field energy transitions can be induced between the two energy states resulting in a perturbation of the net magnetization. This relationship is given by the Lamor equation as follows:

$$
\omega=\gamma^{*} \mathrm{~B}_{0 .}
$$

The spins precess with a characteristic frequency (Lamor frequency or resonance frequency $\omega$ ), thereby initiating a gyroscopic motion, in which additional to the spinning 
motion, the axis of spin itself rotates around a vertical axis. The Lamor frequency depends linearly on the strength of the external field $\left(\mathrm{B}_{0}\right.$, magnetic field strength in Tesla; the higher the magnetic field, the higher the frequency) and the type of atomic nucleus excited (with gyromagnetic ratio $\gamma$ in $\mathrm{MHz} / \mathrm{Tesla}$, constant for a certain type of nuclei). This equation relates the frequency of the resulting signal to the static magnetic field strength. For example, hydrogen-1 will resonate at approximately $128 \mathrm{MHz}$ in a 3.0 Tesla magnet.

The applied alternating magnetic field that is transverse to the main static $\left(\mathrm{B}_{0}\right)$ magnetic field (x,y plane), which is necessary to perturb the spins and oscillates with the Lamor frequencies of the spins, is known as the $\mathrm{B}_{1}$ field (Figure $2 \mathrm{c}$ ). The $\mathrm{B}_{1}$ field perturbs the net magnetization of the sample and creates 'coherence' along a direction in the $\mathrm{x}$ and $\mathrm{y}$ plane (Figure $2 \mathrm{~d})$. The RF field $\left(\mathrm{B}_{1}\right)$ is then switched off and a RF signal is recorded in the receiver coil, when the nuclei relax and return to their initial equilibrium state. The initial amplitude of the detected RF signal is proportional to the number of protons in the sample (proton density). The greater the proton density, the greater the magnetization and the signal detected by the receiver coil.

Within hundreds of seconds after the RF excitation pulse the spin system returns to the equilibrium state. During this time two independent relaxation processes occur, T1 and T2 relaxation. The realignment with the magnetic field is termed longitudinal relaxation and the time in milliseconds required for a certain percentage $(63 \%)$ of the tissue nuclei to realign is called $T 1$. As $T 1$ describes the speed of energy exchange between the spins and their environment (lattice) this is also called spin-lattice relaxation. Spin-spin relaxation refers to the information exchange (phase orientation) between the spins leading to local dephasing following the RF-pulse. This transverse relaxation time is termed T2. The dephasing of the spins is influenced by local inhomogeneities of the static magnetic field as a property of the organ studied and also depending on spatial resolution in MRI (lower resolution causing further signal loss). $T 1$ and $T 2$ relaxation times differ between biological tissues, are influenced by temperature and are independent from one another. The time constant that describes the decay of the transverse component of net magnetization due to both accumulated phase differences and local magnetic field inhomogeneities is called T2*. T2* is always shorter than T2. BOLD-contrast fMRI relies on T2* contrast.

The sequence of RF pulses, that induce electromagnetic echos in order to reconstruct images, is referred to as pulse sequences. The most commonly used MR imaging sequence for fMRI is echo planar imaging (EPI). This pulse sequence produces complete images in as little as $50 \mathrm{~ms}$ and from one selective RF excitation pulse. 
In order to construct 3D images from the brain, it is necessary to identify the excat location of the recorded electromagnetic echos. Therefore, three main priciples are combined: selective excitation of a single slice of the brain (slice excitation), frequency and phase coding. Each of these principles allows the localisation of the origin of the signal within one of the three spatial dimensions $(\mathrm{x}, \mathrm{y}, \mathrm{z})$.

The first step in an imaging sequence is slice selection ( $\mathrm{z}$ gradient). The goal of slice selection is to excite only a particular thin slab of the sample so that signal within that slab can be spatially encoded. By introducing a static gradient along the slice slection axis (e.g., $G_{z}$ ), the Lamor frequencies of all spins in the slice can be tuned to match the frequency of the excitation pulse. The slice chosen by the selection process is defined by its location, orientation, and thickness.

Once spins are excited within the desired slice, they can be spatially encoded so that MR signal from different parts of the image can be resolved. A unique frequency is assigned to all voxels within the slice, in a process known as frequency and phase encoding, to facilitate reconstruction of the signal using the Fourier transform (FT). Therfore, a gradient magnetic field that differs across two dimensions (e.g., $G_{x}, G_{y}$ ) is applied to the sample. These gradients influence the individual spin phases for different voxels. First the phaseencoding gradient ( $\mathrm{y}$ gradient) is energized momentarily, allowing the columns to be identified within the slice. Finally, the frequency-encoding or read gradient (x gradient) is applied during signal acquisition, which identifies the rows in each column. Together, the $\mathrm{x}$ and $y$ gradients allow precise determination of where the contribution to the MR signal from each voxel originated within the transverse imaging section.

The acquired signals are recorded in $\mathrm{k}$-space (with $\mathrm{k}$ denoting wave number, by convention). The $\mathrm{k}$-space representation of the $\mathrm{MR}$ signal is decoded into the magnetization at each spatial location by using the FT, a well established mathematical tool for constructing a spatially informative image. An inverse FT can convert k-space into an image, a process known as image reconstruction. Conversely, a forward FT can convert image-space data into k-space data.

\section{$\underline{2.3 \text { Physiological principles }}$}

The emergence of fMRI methodology is fundamentally based on the fortuitous presence of an endogenous contrast agent, paramagnetic deoxyhaemoglobin, circulating in the brain and the tight coupling between neuronal activation and haemodynamic/metabolic responses. The fMRI method takes advantage of the fact that oxyhaemoglobin (the 
oxygenated form of haemoglobin in the blood) has different magnetic properties than deoxyhaemoglobin (haemoglobin that has donated its oxygen). Thus, fMRI makes use of the different magnetic properties of oxygenated and deoxygenated haemoglobin to construct images based upon BOLD contrast (Ogawa et al., 1990; Ogawa et al., 1992; Kwong et al., 1992).

BOLD contrast is a consequence of a series of indirect effects (Figure 3). It results from changes in the magnetic properties of water molecules, which in turn reflect the influence of paramagnetic deoxyhaemoglobin, which is a physiological correlate of oxygen consumption, which itself is a correlate of a change in neuronal activity evoked by sensory, motor, and/or cognitive processes.

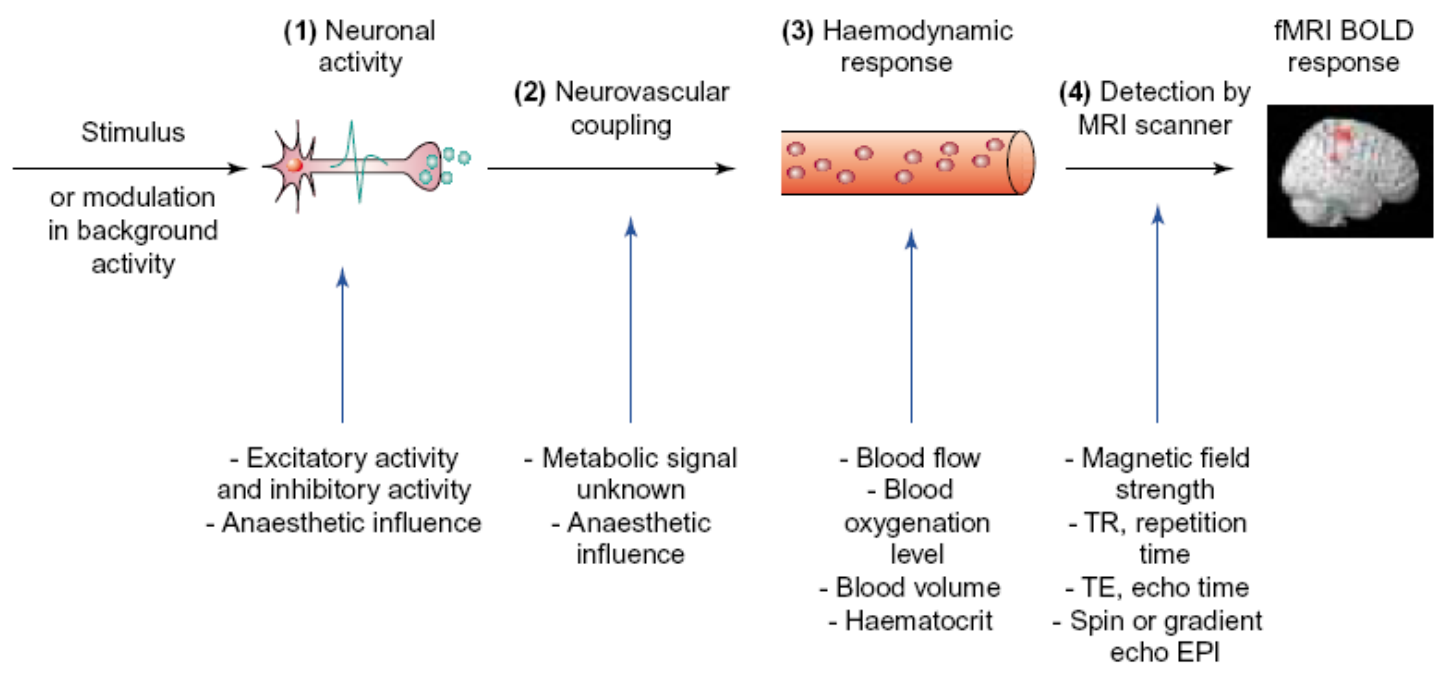

Figure 3

The BOLD signal has several constituents: (1) the neuronal response to a stimulus or background modulation; (2) the complex relationship between neuronal activity and triggering a haemodynamic response (termed neurovascular coupling); (3) the haemodynamic response itself; and (4) the way in which this response is detected by an MRI scanner (Arthurs \& Boniface, 2002).

The activation of neurons and establishment of ion potentials in the cells of the brain all require a supply of energy. This is supplied in the form of adenosine tri-phosphate (ATP) generated in the mitochondria within cells. Under normal conditions the formation of ATP via glucose consumption requires oxygen, and this oxygen is supplied by blood perfusing the tissue. Since oxygen is not very soluble in blood, it is transported bound to the large iron-containing molecule, haemoglobin (Figure 4). Because of the magnetic properties of the iron atoms in haemoglobin, the relaxation time of the blood is reduced.

When oxygen is bound to haemoglobin, the molecule alters from being paramagnetic (having a significant magnetic effect on its environment) to being diamagnetic (having 
little effect). Such oxygen dependence makes haemoglobin a sensitive marker to the level of blood oxygenation and consequently to neuronal activity. If the oxygenation level of the blood decreases (which means that the level of deoxyhaemoglobin increases) it causes the T2* of blood, and to a lesser extent the T2 of blood, to decrease also, resulting in slightly lower signal in a $\mathrm{T} 2 *$-weighted image. Conversely, if the blood oxygenation level rises, then the $\mathrm{T} 2 *$ value increases, resulting in higher signal in a $\mathrm{T} 2 *$-weighted image. The signal seen in MR images upon neuronal activation is a positive signal change, representing a decrease in the concentration of deoxyhaemoglobin.

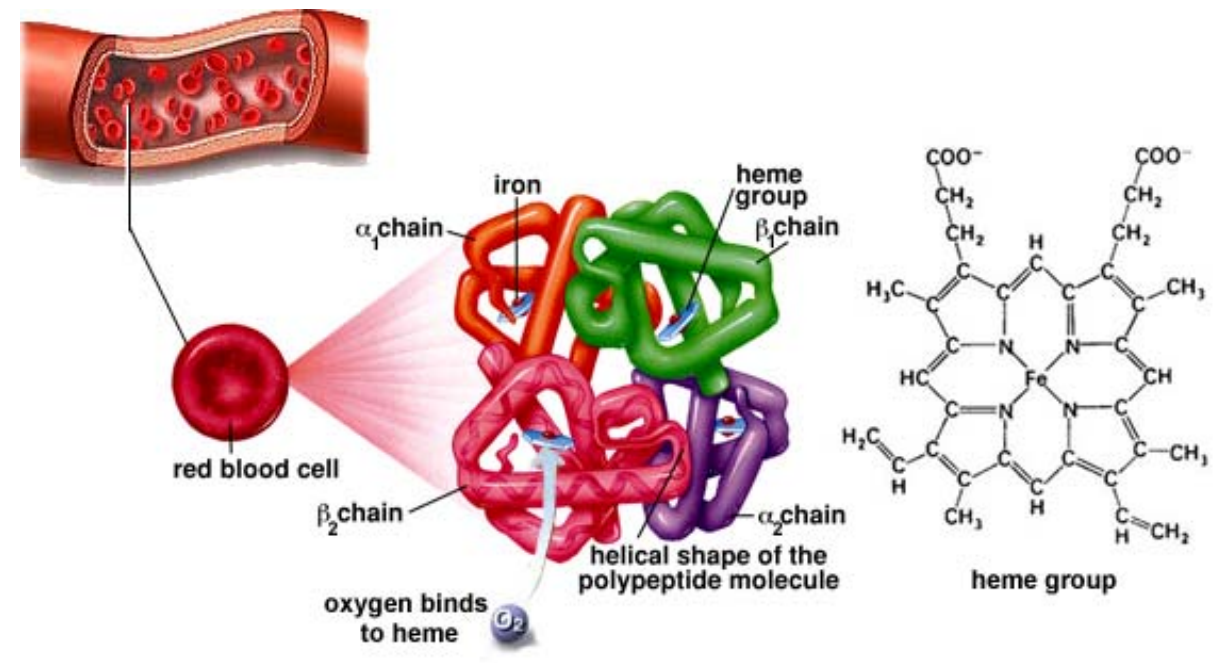

\section{Figure 4}

Haemoglobin (Hgb) is the most important component of red blood cells. It is coupled to four porphyrins or heme moities, which bind oxygen. The globin portion of Hgb consists of four polypeptide chains ( $\alpha$ chains and $\beta$ chains) arranged in pairs forming a tetramer. Each globin chain is covalently attached to a heme moiety. The bonds between $\alpha$ and $\beta$ chains are weaker than between similar globin chains, forming a natural cleavage plane, the $\alpha_{1} \beta_{2}$ interface, important for oxygen binding and release. The binding of oxygen rotates the globin chains, moving the $\beta$ chains together and sliding the $\alpha_{1} \beta_{2}$ interfaces apart thus increasing the oxygen affinity of Hgb (adapted from http://www.mhhe.com/biosci/ap/dynamichuman2/content/cardio/visuals.mhtml).

The typical fMRI BOLD response is schematically divided into three epochs (Figure 5). First, immediately after electrical activity commences there may be a brief period of approximately $0.5-1 \mathrm{~s}$ during which the MRI signal decreases slightly below baseline $(\sim 0.5 \%)$. This is a very subtle effect and is often not seen at conventional magnetic field strengths. This first epoch has become known as the 'initial dip' and is still controversly discussed. Subsequently, the BOLD response increases, yielding a robust 'positive BOLD response' which peaks $5-8 \mathrm{~s}$ after the stimulus commences. It is this positive BOLD response that is used in most fMRI experiments. During the early positive BOLD response, 
another common feature observed in fMRI experiments is a short 'overshoot' period. Finally, upon cessation of the stimulus, there is a return of the BOLD response to baseline, often accompanied by a 'post-stimulus undershoot', during which the response passes through baseline and remains negative for several tens of seconds. Eventually, the response returns to baseline. In a typical fMRI experiment, the magnitude of the BOLD signal is quite small, in the order of $0.1 \%$ to $5 \%$ of signal change.

Although it is known that the fMRI signal is triggered by the metabolic demands of increased neuronal activity, the details of this process are only partially understood. The relationship between the fMRI data and the neuronal activity depends on several factors (Heeger \& Ress, 2002).

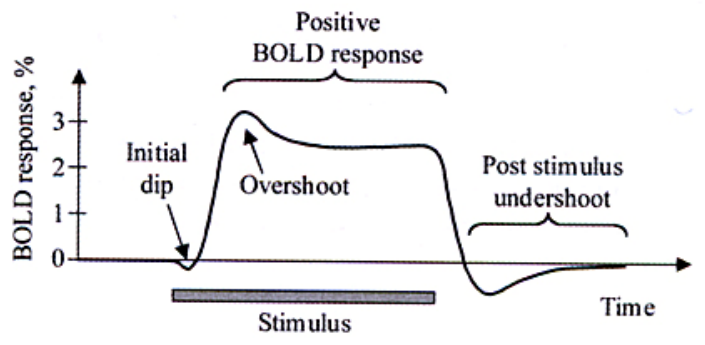

\section{Figure 5}

Schematic representation of the common features of the fMRI BOLD response to a period of neuronal stimulation. During the first epoch a small negative 'initial dip' may be observed. Subsequently, a more robust 'positive BOLD response' is obtained. Following cessation of the stimulus a return to baseline accompanied by a 'post-stimulus undershoot' is often seen (from Hoge \& Pike, 2001).

Most importantly, the relationship between fMRI and neuronal responses depends on the fMRI acquisition technique. Most fMRI experiments measure a BOLD response, which provides a mixed signal that depends on blood flow (CBF), blood volume (CBV) and blood oxygenation and is referred to as the haemodynamic response to activation. Various processes have been proposed to cause the haemodynamic response (Magistretti \& Pellerin, 1999; Villringer \& Dirnagl, 1995; Bonvento et al., 2002). One concept is based on the idea that the response might be triggered by lactate (released by the astrocytes), implying a tight link between the fMRI signal and synaptic activity. Another model proposes, that the haemodynamic response is triggered by a diffusible by-product of neuronal spiking, for example by nitirc oxide. Another possibility is that the haemodynamic response might be triggered within the blood vessels themselves, in 
response to transient decreases in oxygenation (the initial dip). All these three processes might also operate together and with other mechanisms.

Furthermore other critical factors influencing the relationship between measured fMRI signal change and neuronal response are the stimulation protocols, as well as the fMRI data analysis method used. Predominantly, a model with prior knowledge about the delay and dispersion in haemodynamic coupling between neuronal response and obtained fMRI signal change is used assuming the haemodynamic response being shaped by two gamma distributions, fitting the initial rise and the subsequent undershoot, respectively. Most fMRI studies use statistical parametric mapping (SPM) for the analysis of functional data and the obtained haemodynamic response is given by convolving the evoked neuronal response with the haemodynamic response function (HRF). It relies on an impulse response function (response to a single impulse, measured at a series of times after the input), characterizes the input-output behaviour of the system and restricts the sort of inputs that can excite a response.

\subsection{Data analyses}

In a typical fMRI session a functional volume is acquired every few seconds (MR volumes are often also referred to as 'images' or 'scans'). During one volume a certain number of slices is acquired depending on the experimental design and the region of the brain relevant to the research hypothesis. Over the course of the experiment, 100 volumes or more are typically recorded. Because the images are taken using an $\mathrm{T} 2 *$-weighted MR sequence which is sensitive to changes in BOLD response, parts of the images taken during stimulation may show increased intensity, compared with those taken whilst at rest. The parts of these images which show increased intensity should correspond to the brain areas which are activated by the stimulation. The goal of fMRI analysis is to detect, in a robst, sensitive, and valid way, those parts of the brain which show increased intensity at the points in time when stimulation was applied.

A single slice or image is made up of individual cuboid elements called voxels (Figure 6). An fMRI data set from a single session can either be thought of as $t$ volumes, one taken every few seconds, or as $v$ voxels, each with an associated time series of $t$ time points (Figure 7).

After an fMRI experiment has been designed and conducted, the resulting data must be passed through various analysis steps before activation maps at individual or multi-subject level can be interpreted. The analysis of fMRI data appears to be a critical, time-consuming 
and sometimes controversial part of fMRI experiments as a number of different software tools are available, each having strengths and weaknesses (AFNI, Brain Voyager, SPM, Freesurfer, FSL, etc.). For the analysis of the fMRI data presented in this thesis Brain Voyager QX has been used.

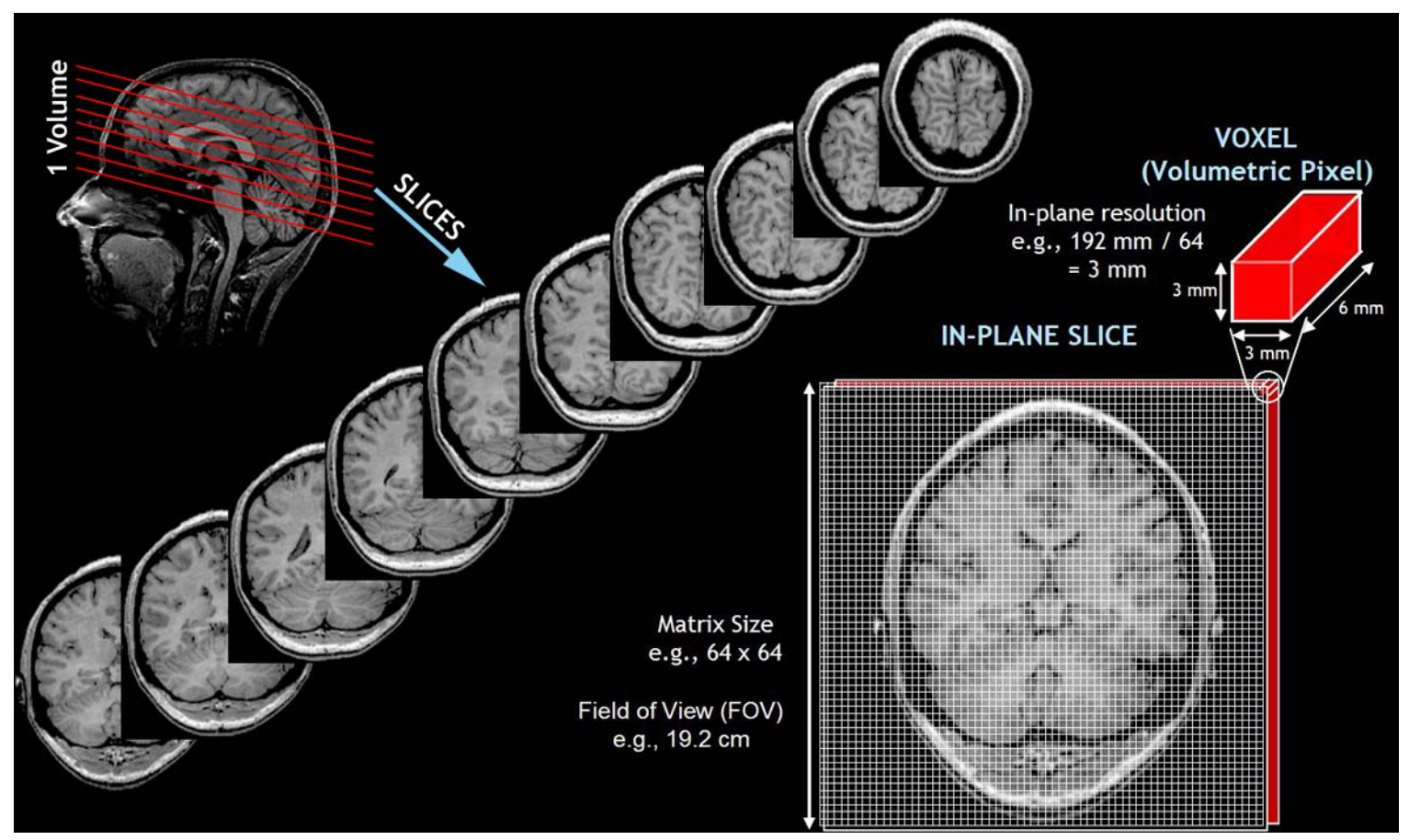

Figure 6

MR signals are collected simultaneously from a large volume of the brain, which is called volume imaging. Each volume or measurement consists of the number of slices, which are separarted by each other by the interslice space or gap. Each slice consits of voxels, which are the element of three-dimensional space corresponding to a pixel for a given slice thickness and which constitute the matrix size. The FOV and matrix size determine the pixel-size and therefore reflect area of anatomical region displayed.

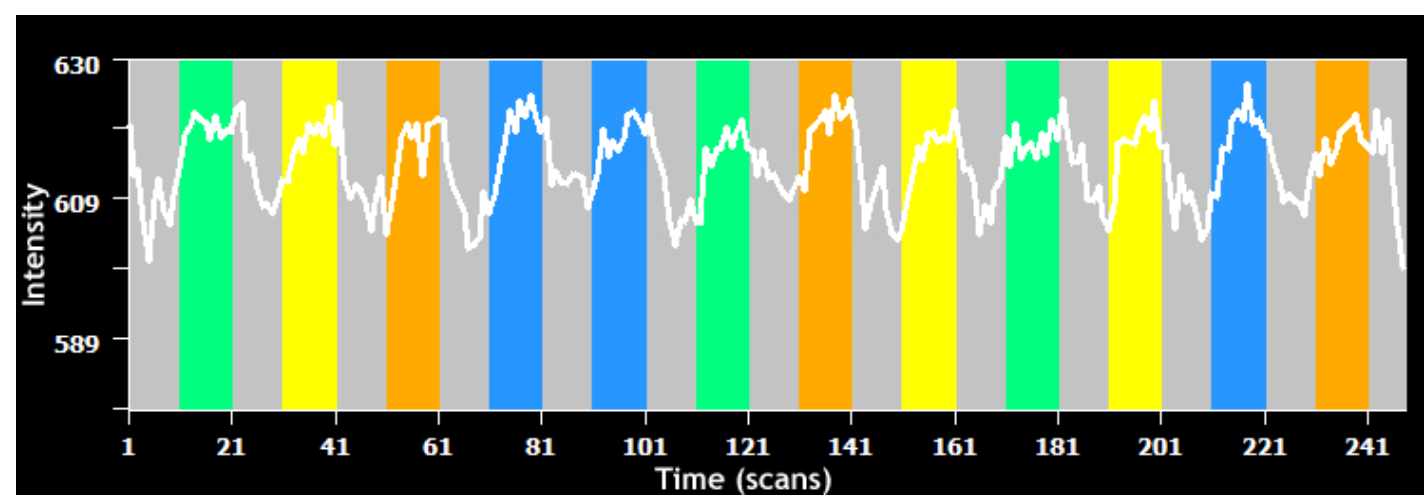

\section{Figure 7}

An example time series at a strongly activated cluster of voxels from an attention experiment. Periods of stimulation (green, yellow, orange, blue) are alternated with periods of rest (grey). A complete stimulation-rest cycle lasts 20 scans. 
This chapter gives a brief overview of the various analysis steps, covering the analysis of the anatomical and functional data thereby describing pre- and postprocessing steps.

\subsubsection{Analysis of anatomical data}

Initially, the high-resolution 3D T1-weighted dataset (anatomical dataset), usually obtained at the beginning of the scan session, is reconstructed to visualize the functional data in Talairach space and to create head and cortex reconstructions. In a first step the cerebrum is translated and rotated into the AC-PC plane (AC: anterior commissure; PC: posterior commissure). Therefore the dataset is rotated axially, coronally and transversally to obtain a symmetric reconstruction in all three planes (Figure 8). In a second step the cerebrum is transformed into the space of Talairach and Tournoux (1988), which is a commonly used 'standard' space for reporting locations of activated brain regions and for averaging data across subjects. After the dataset has been transformed into the AC-PC plane, eight other reference points are specified to identify the borders of the cerebrum in order to fit the size of the brain into standard space (Talairach space).

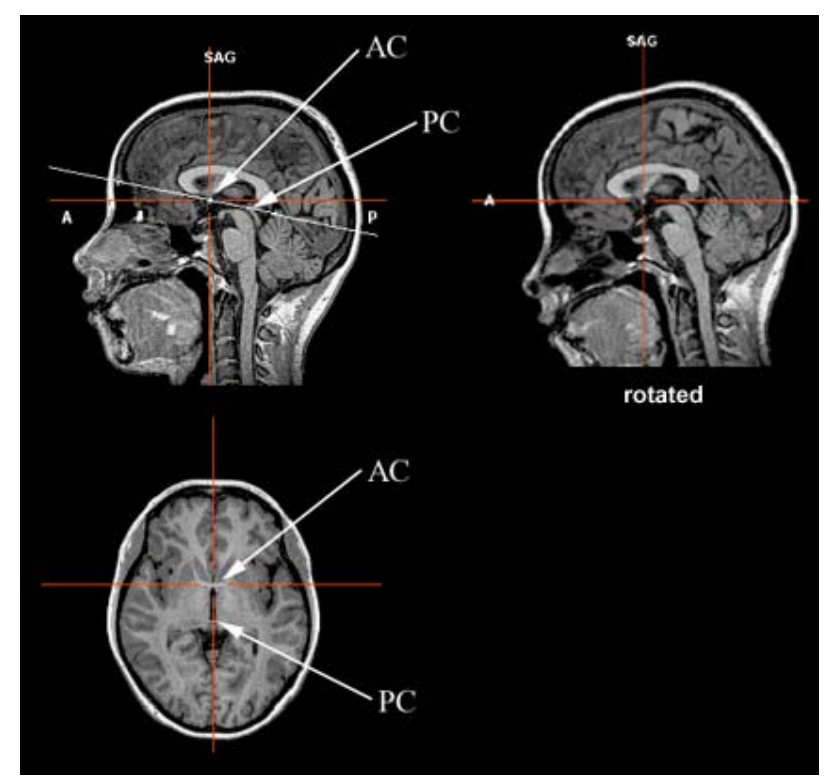

\section{Figure 8}

Rotation of the anatomical dataset along the AC-PC plane.

Besides standard surface views, several other types of rendered images are available. To illustrate activity that lies within deep cortical sulci, fMRI data are displayed on inflated brains or flat maps (Figure 9). 
As the cortex is basically a single folded sheet about $5 \mathrm{~mm}$ in depth, the inflated brain expands the cortical surface like a balloon while maintaining its basic shape. A flat map is obtained by cutting the inflated surface at different points (in the present study, the surface was cut along the calcarine sulcus) and laying out the cortical sheet in two dimensions. Since no changes in depth are visible in these techniques, the original gyral and sulcal patterns are marked using different colours or brightness levels. However, the transformation of a 3D object like the brain into a two-dimensional (2D) map is by necessity associated with local distortions, which represent a significant problem in the generation of flat maps of the brain.
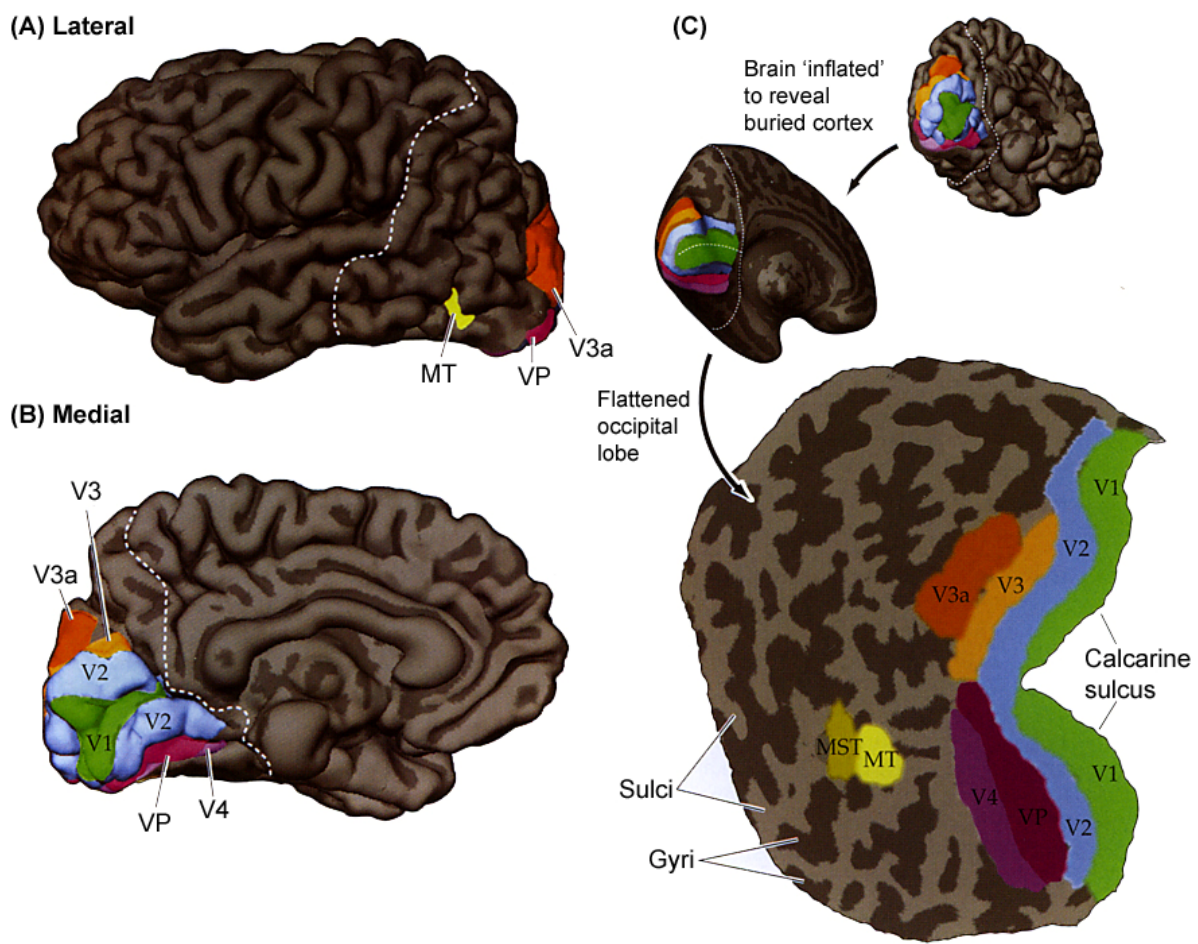

\section{Figure 9}

Flat map views of the brain surface illustrating the retinotopic organization of the visual cortex. Areas of activation are first displayed on lateral (a) and medial (b) views of the visual cortex, then the cortical surface is inflated, cut and flattened (c) to show the spatial distribution of active regions (from Huettel et al., 2001). 


\subsubsection{Analysis of functional data}

Before the main part of the analysis a number of preliminary steps, so called preprocessing, are performed to prepare the data for statistical analysis. The following section describes the most important preprocessing steps as performed by using BrainVoyager QX.

Once the data has been acquired, the preprocessing starts by reconstructing the raw ' $\mathrm{k}$ space' data into brain images (fMRI images; functional data). The next step applied is motion correction which is a very important step during preprocessing. The basic problem introduced by head motion is that fMRI analyses assume that each voxel represents a unique part of the brain; if the subject's head moves, then each voxel's time course is derived from more than one brain location which represents a serious confound in fMRI studies. During motion correction each volume is transformed using rotation (rotating the entire image volume through the $\mathrm{x}-, \mathrm{y}-$, and $\mathrm{z}$-planes) and translation (i.e., moving the entire image volume along $\mathrm{x}-, \mathrm{y}-$, and $\mathrm{z}$-axes) so that the image of the brain within each volume is aligned with the one in every other volume.

Afterwards filters are used to remove or retain different frequency components that are present in a composite signal. Low-pass spatial filters are employed to reduce the highfrequency spatial components and "blur" the images (spatial smoothing). The most common blurring technique is the introduction of a Gaussian filter. A Gaussian filter has the shape of a normal distribution. When a Gaussian filter is applied, it effectively spreads the intensity at each voxel in the image over nearby voxels. Therefore the narrowness or wideness of the filter refers to the distance of its effect. Spatial filter width for fMRI data is generally expressed in millimeters at half of the maximum value (full-width-halfmaximum, FWHM).

The use of temporal filters can substantially improve the quality of fMRI data by improving functional signal-to-noise ratio (SNR) (temporal filtering). In order to keep information about changes in the data that occur at the task frequency and to minimize changes in the data that occur at other frequencies, it is necessary to reduce the contribution of noise isolated in particular frequency ranges. Therefore temporal filters that selectively attenuate certain frequency ranges are used. A low-pass filter leaves low frequencies intact while attenuating high frequencies, and a high-pass filter stops only low frequencies. High-pass filtering of the data can also remove slow driftlike trends, which is 
achieved by filtering each voxel's time series by linear or non-linear tools (Linear Trend Removal).

The purpose of the preprocessing is to remove various kinds of artefacts in the data, and to condition the data, in order to maximize the sensitivity of later statistical analysis, and also, in some situations, to increase the statistical validity. After the preprocessing the fMRI images are aligned to the $3 \mathrm{D}$ anatomical dataset, so that activations can be viewed in the context of a high quality brain image. Finally, when the registration with the anatomical dataset is completed, the functional data are transformed into the standard coordinate system of Talairach and Tournoux (1988).

After the preprocessing steps, statistical analysis is carried out to determine which voxels are activated by the stimulation. In fMRI studies each voxel's time series is commonly analyzed independently (univariate analysis) using the general linear model approach (GLM). General linear modelling sets up a model and fits it to the data. If the model is derived from the timing of the stimulation that was applied to the subject in the MRI scanner, then a good fit between the model and the data means that the data was probably caused by the stimulation. The formula for a linear model is:

$$
\mathrm{y}=\mathrm{a}_{0}+\mathrm{a}_{1} \mathrm{x}_{1}+\mathrm{a}_{2} \mathrm{x}_{2}+\ldots+\mathrm{a}_{\mathrm{n}} \mathrm{x}_{\mathrm{n}}+\mathrm{e}
$$

The basic idea behind a linear model is that the observed data $(y)$ is equal to a weighted combination of several model factors $\left(x_{i}\right)$ plus an additive error term $(e)$. The parameter weights $\left(a_{i}\right)$ indicate how much each factor contributes to the overall data. The term $a_{0}$ reflects the total contribution of all factors that are held constant throughout the experiment. For fMRI data, this would include the raw signal recorded in particular voxels in the absence of BOLD activation, as well as any activity that is constant throughout the experiment.

When the linear model is applied to fMRI data, the experimental data are represented as a 2D matrix consisting of $n$ time points by $v$ voxels (Figure 10). In the GLM the values for the parameter weights and error term are calculated independently for all voxels. The voxels are arranged along one dimension for ease of calculation. The design matrix, which specifies the linear model to be evaluated, consists of $M$ model factors, each $n$ time points in lengths. The parameter matrix contains $M$ rows and $V$ columns, such that each cell indicates the amplitude of one of the model factors for a given voxel. Finally, the error term is an $n$-by- $V$ matrix. 
In order to get the best possible fit of the model to the data, the 'stimulus function' (which is often a sharp on/off waveform) is convolved with the HRF. This process mimics the effect that the brain's neurophysiology has on the input function (the stimulation). The haemodynamic response is a delayed and blurred version of the input time-series, so a mathematical operation is applied to the stimulus function to take the square wave input and create a delayed version, which will better fit the data.

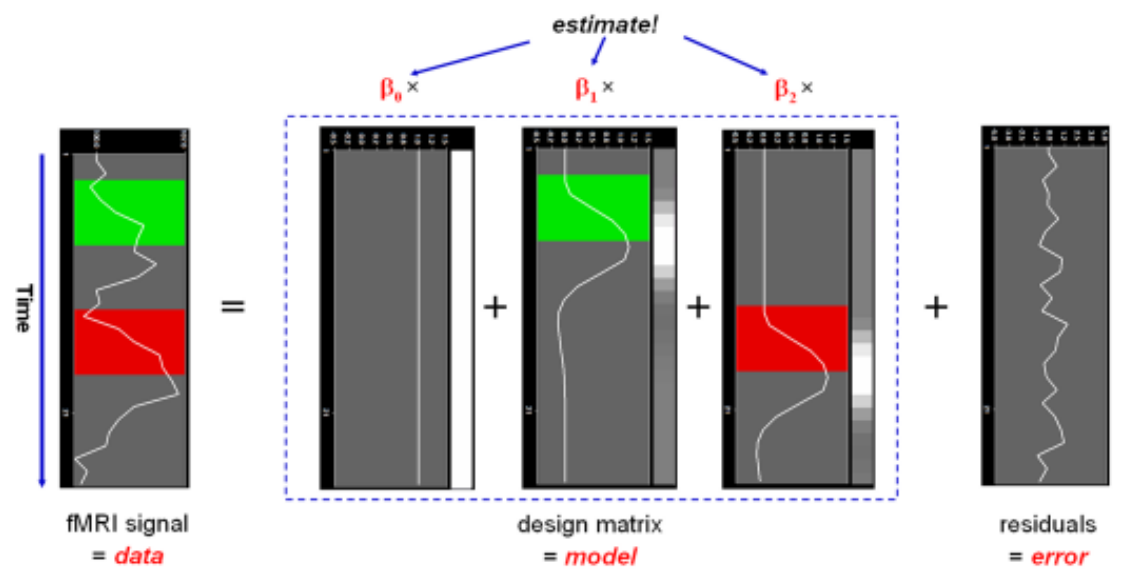

\section{Figure 10}

Basic principles of the general linear model in fMRI. The general linear model attempts to find the set of experimental parameters $(\mathrm{x})$ for a design matrix $(\beta)$ that best accounts for the original data (from www.brainvoyager.com/bvqx/doc/UsersGuide/WebHelp/BrainVoyagerQXUsersGuide.htm).

Since fMRI data consist of many time points, the residual error for a given voxel must be combined across all time points into a single value, which is done by the cost function of least squares error (sum of all squared residuals). To test the significance of a model factor for a given voxel, the amplitude of its associated parameter is divided by the residual error. Under the null-hypothesis, this quantity should follow the $F$ distribution and therefore its statistical significance can be evaluated as a function of the available degrees of freedom. With the known degrees of freedom, an $F$ test can be converted in an error probability value $p$. A high $F$ value (low $p$ value) indicates that one or more experimental conditions substantially modulate the data time course. However, the $F$ statistic does not allow to assess which individual conditions differ significantly from each other. Therefore, comparisons between conditions can be formulated as contrasts. A contrast is simply a linear mixture of parameter estimates. The $T$ statistic allows to test the null hypothesis that some contrast (e.g. a subtraction) of the estimates is zero. The $T$ statistic is obtained by division of the contrast (specified by the contrast weights) of the parameter estimates by its 
standard error. After the $F$ or $T$ statistic is applied, a map containing only voxels that survived the chosen threshold is computed indicating the voxels with significant activation. Finally, these maps are projected onto the 3D anatomical Talairach transformed dataset displaying the significant responses as coloured blobs of activation (Figure 11).

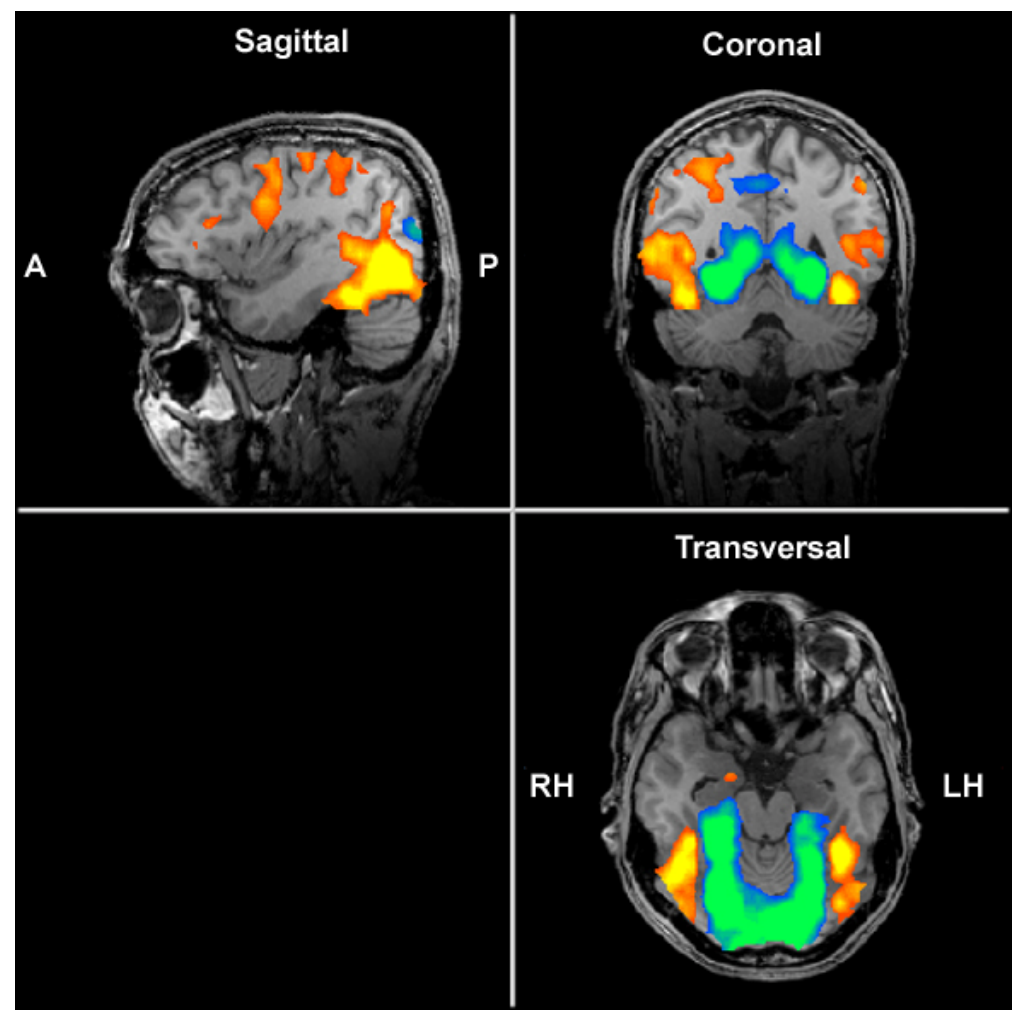

\section{Figure 11}

Activation map of an experiment to localize face-responsive regions (houses and faces were presented in a blocked design). The yellow-red blobs represent increased activity in response to faces whereas the green-blue blobs indicate enhanced responses to houses. A: anterior. P: posterior. RH: right hemisphere. LH: left hemisphere.

An important issue in fMRI data analysis is the specification of an appropriate threshold for statistical maps. There are a several ways of carrying out thresholding and to correct for multiple comparisons (Bonferroni correction, Gaussian Random Field theory, cluster-size thresholding, False Discovery Rate, etc.). In this thesis I used the False Discovery Rate (FDR) (Genovese et al., 2002) for thresholding as it is the default method used in Brain Voyager QX.

There are two common statistical approaches for fMRI studies, fixed-effects analysis (FFX) and random-effects analysis (RFX). FFX analysis assumes that the experimental effect is fixed, or constant across subjects, with differences between subjects caused by random noise. 
While in a FFX analysis the obtained group results can not be generalized to the population level since the data of all subjects is concatenated and analyzed as if it stems from a single subject, the RFX analysis allows inference to the population from which the sample of subjects have been drawn. The RFX analysis explicitly models the inter-subject variability in treating the effect of the experimental manipulation as variable across subjects, so that it could have a different effect upon different subjects. The error variance in a FFX analysis is, thus, estimated by the variability across individual measurement time points while in a RFX analysis, the error variance is estimated by the variability of subject-specific effects across subjects.

While voxelwise analyses determine significant effects in many different voxels, often encompassing the entire brain, some hypotheses require a more targeted analysis approach, especially when a particular brain region is related to them. Therefore, a region-of-interest (ROI) analysis is performed which evaluates statistical tests on a predetermined collection of voxels. The ROI is considered to be a homogenous and indivisible unit, at least for the purposes of the ROI analysis, and can be either created on an anatomical or a functional basis.

\section{$\underline{2.5 \text { General MR parameters }}$}

In this thesis, MRI was performed at 3 Tesla (Magnetom Trio, Siemens, Erlangen, Germany) in the MR-Research in Neuorlogy and Psychiatry at the University Clinic of the Georg August University of Goettingen.

Initially, a high-resolution 3D T1-weighted dataset was acquired (176 sagittal sections, $\left.1 \times 1 \times 1 \mathrm{~mm}^{3}\right)$. For fMRI a T2*-weighted, gradient-echo echo planar imaging technique recording 22 sections of $4 \mathrm{~mm}$ thickness oriented roughly parallel to the calcarine sulcus at an in-plane resolution of $2 \times 2 \mathrm{~mm}^{2}$ was used (repetition time $=2000 \mathrm{~ms}$; echo time $=36 \mathrm{~ms}$; field-of-view $=192 \times 256 \mathrm{~mm}^{2}$ ).

Preprocessing of fMRI data included 3D-motion-correction, temporal high pass filtering (3 cycles/run), linear trend removal, and transformation into the space of Talairach and Tournoux (1988). 


\section{Selective visual attention}

„Everyone knows what attention is. It is the taking possession of the mind, in clear and vivid form, of one out of several possible objects or trains of thought. Focalisation, concentration of consciousness are of its essence. It implies withdrawal from some things in order to deal effectively with others."

William James

The concept of attention as crucial to human behaviour and performance extends back to the start of experimental psychology. The quote above by William James (1950) more than 100 years ago captures the very essence of attention without recourse to scientific jargon. Although precise definitions of attention are somewhat lacking, it may be thought of in the simplest terms as the appropriate allocation of processing resources to e.g., relevant objects, locations or even moments in time.

Attention is thought to comprise several sub-processes that perform different but interrelated functions: (a) attentional orientation (the simple direction of attention to a particular stimulus); (b) selective (or focused) attention (giving attentional priority to one stimulus in favour of another); (c) divided attention (dividing attention between two or more different stimuli); and (d) sustained attention (attending to one stimulus over an increasing period of time) (Posner \& Petersen, 1990; Kahneman, 1973; Posner \& Boies 1971).

In ordinary language the term attention is associated with several meanings, the most common of which are mental effort and selective processing (Posner \& Boies, 1971; Johnston \& Heinz, 1978). The view of attention as mental effort derives from the basic assumption that the processing capacity of the visual system is limited in some central mechanism (Kahneman, 1973; Shiffrin \& Schneider, 1977). This mechanism is associated with consciousness and controlled processing and it delimits the extent to simultaneously process different sources of information (divided attention). As mentioned earlier the processing of different sources of information at the same time and the ability to filter out 
unwanted or irrelevant information, is referred to as selective attention. In nature these sources are internal (memory and knowledge) as well as external (environmental objects and events).

The selective function of attention is assumed to be manifest in three main forms: simple selection, preparation, and maintenance (LaBerge, 1995). Simple selection is typically brief in duration (searching displays or reading words) and helps to select a target item from distracters in a cluttered visual scene (Treisman \& Gelade, 1980; Yantis, 1993). During the preparatory state, attention to a specific object, attribute or spatial location is sustained over a preparatory period of time (e.g. between a cue and a target) in order to respond more effectively to an expected stimulus event (Posner, 1980; Eriksen \& Yeh, 1985; Downing, 1988). Maintenance refers to cases in which attention to a selected entity is sustained over a period of time and helps to maintain particular cognitive activities for their own sake (LaBerge, 1995).

New developments in neuroscience, including functional brain imaging with positronemission tomography (PET), functional magnetic resonance imaging (fMRI), and neurobiological measures, including single-cell recording in animals, scalp recording of event-related potentials (ERPs) and magnetoencephalography (MEG) in humans have made crucial contributions in investigating the foundations of human selective attention. The most influential psychological models of attention that date back in the 1950s provided a parallel-then-serial feedforward architecture of selective attention where selectivity arises at one particular point (Broadbent, 1958; Deutsch \& Deutsch, 1963; Treisman, 1960, 1969, 1988). Recent research overturns many fundamental assumptions behind traditional information-processing models of selective attention. From a more neuronal perspective than the original information-processing models, the 'biased competition' model proposed by Desimone and Duncan (1995; Duncan, 1996) posits that attention coordinates selective information processing in the visual system. Because of the limited processing capacity of the visual system, multiple objects present at the same time will compete for neuronal processing resources. This constant competition can be influenced by stimulus-driven ('bottom-up') mechanisms, such as stimulus salience, or by 'top-down' cognitive mechanisms, as selective attention. Neurobiological measures revealed that initial perceptual processing (extracting basic attributes such as location, orientation, colour, or motion) is not unaffected by attention (Eason et al., 1969; Van Voorhis \& Hillyard, 1977; Hopfinger et al., 2000; Luck et al., 2000; Heinze et al., 1994; Mangun, 1995; Bushnell et al., 1981; Moran \& Desimone, 1985; Maunsell, 1995; Kastner 
\& Ungerleider, 2001; Shulman, 1990; Freeman et al., 2001). Thus, instead of promoting the idea that attentional modulation arises at just one particular stage, it may therefore arise in a wide-spread manner, affecting multiple levels of sensory processing to different degrees. It has been demonstrated that even primary visual cortex can be modulated by spatial attention in humans (Brefczynski \& DeYoe, 1999; Gandhi at al., 1999; Martinez et al., 1999; Somers et al., 1999; Kanwisher \& Wojciulik, 2000; Kastner \& Ungerleider, 2001). Moreover, the work on preparatory effects, which means that attention is directed covertly to a particular location where the occurance of visual stimuli is expected, clearly demonstrates top-down and not feedforward influences (Kastner et al., 1999).

The neuronal basis of attention has been viewed in terms of large-scale neuronal networks corresponding to areas active during imaging tasks that require attention. It has been proposed that the sources of attention form a specific system of anatomical areas, which can be further broken down into three networks related to different functions and aspects of attention (Posner \& Petersen, 1990; Fan et al., 2002, 2005). These networks carry out the functions of alerting, orienting and executive attention (Posner \& Rothbart, 2007).

Alerting is defined as achieving and maintaining a state of high sensitivity to incoming stimuli. The alerting system has been associated with thalamic as well as frontal and parietal regions of the cortex. Orienting is the selection of information from sensory input. The orienting system has been associated with areas of the parietal lobe, including the superior parietal lobe and temporal parietal junction, and frontal lobes. The orienting system can be further distinguished as being used for overt (when the body, head, or eyes are moved) or covert (when no overt movement is made) shifts of attention. Furthermore, such shifts of attention appear to involve three processing subcomponents: in order to shift attention one must first disengage it from its current location, move it, and then engage attention at a newly specified location. The executive attention system involves mechanisms for monitoring and resolving conflict among thoughts, feelings and responses. It has also been suggested that the executive attention network is involved in selfregulation of positive and negative affect as well as a wide variety of cognitive tasks underlying intelligence (Duncan et al., 2000). The executive network is based on the anterior cingulate and prefrontal areas. These specific attentional networks do not generally operate independently. Interactions in between the different networks have been observed (Callejas et al., 2004, 2005; Fan et al., 2002). The executive network is inhibited by the alerting network, whereas the orienting network raises the efficiency of the executive network and alertness increases orienting. 
As mentioned above the mechanisms that orient attention not only shift one's body, head, and eyes so that a specific stimulus can be fixed, but also can shift the locus of attention covertly. This attentional selection mechanism has been characterized by a broad array of metaphors, of which the most relevant ones for this thesis will be presented in the last section of this chapter. In order to understand the neuronal mechanisms underlying visual selective attention, a model of the functional architecture of the visual system is required, in which the processes of attentional selection are implemented. Therefore, first the organization of the visual cortex is described, and then the evidence regarding different models of spatial attention is reviewed.

\section{$\underline{3.1 \text { Organization of visual cortex }}$}

There is converging evidence from single-cell physiology studies in primates and functional imaging studies in humans that selective attention modulates neuronal activity in the visual system (Luck et al, 1997; Kastner \& Ungerleider, 2001; Kanwisher \& Wojciulik, 2000). In the past decade the use of functional neuroimaging, particularly fMRI, has dramatically increased our knowledge of the functional organization of the human visual cortex. In order to account for the multiplicity of visual areas, two main principles have been suggested: hierarchical processing and functional specialization.

Hierarchical processing is based on the idea that visual perception is achieved via a gradual stage wise process. Information is first represented in a localized and simple form and, through a sequence of processes, is transformed into more abstract, holistic, and even multimodal representations (DeYoe \& Van Essen, 1988). The second principle, functional specialization, suggests that there exist specialized neuronal pathways that process information about different aspects of the visual scene. Two main functionally specialized processing streams have been proposed (Mishkin et al., 1983; Goodale \& Milner, 1992). Both pathways originate in the primary visual cortex (V1) and both are composed of multiple areas beyond V1. The occipito-parietal pathway or dorsal stream, also referred to as the "where" or "action" stream, has been related to spatial localization (or visually guided action). The occipito-temporal pathway or ventral stream, also known as the "what" stream, has been associated with object and form recognition.

The primary visual cortex receives most of the efferent fibers from the lateral geniculate nucleus (LGN) and is located almost entirely medially on either side of the calcarine sulcus in the occipital lobe. It is referred to as area V1 or Brodmanns area (BA) 17 and also called the striate cortex because a thick stripe of afferent inputs, the stripe of Gennari, runs 
through it. The primary visual cortex is surrounded by two other purely visual areas, area 18 (the prestriate cortex), and area 19 (medial temporal cortex). Area 18 has been subdivided into areas V2 through V4, and area 19 is area V5/MT. Localization of visual inputs is achieved through projection to the dorsal and ventral processing streams.

The primary visual cortex contains an ordered map of the visual field. Each hemifield is represented on the contralateral hemisphere. On each side, the upper quadrants are represented below the calcarine sulcus, while the lower quadrants are represented above the calcarine sulcus. In each hemisphere, a disproportionately large anatomical area of the visual cortex is devoted to the representation of the fovea, which is the retinal area of greatest acuity (Figure 12).
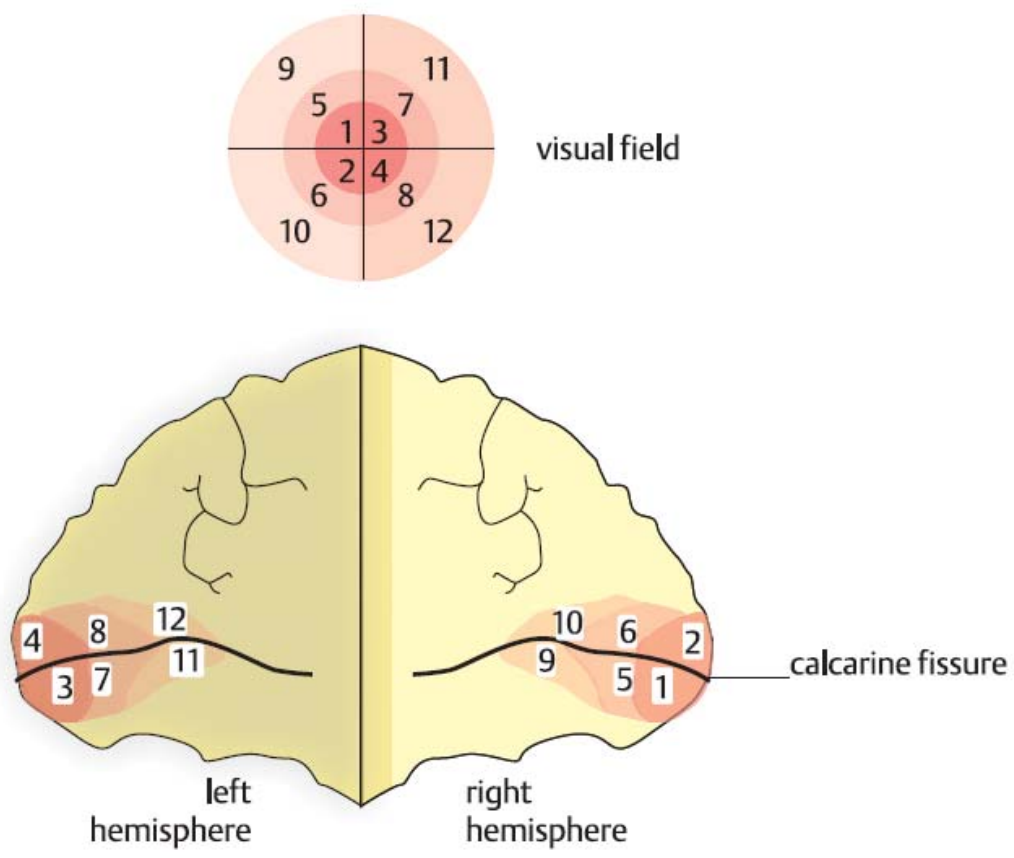

Figure 12

Map of the visual field in primary visual cortex (from Greenstein \& Greenstein, 2000).

Visual field topography is used to identify and map visual areas (DeYoe et al., 1996; Sereno et al., 1995, 2001; Wandell, 1999). Mapping from the retina to the primary visual cortex is topographic in that nearby regions on the retina project to nearby cortical regions. This general organizational feature of the central visual system is called retinotopy. Mapping between the retina and the cortex can be described best as a log-polar transformation, in which standard axes in the retina are transformed into polar axes in the cortex: eccentricity (distance from fovea) and polar angle (angle from horizontal axis). In order to determine the topographical cortical representations, subjects are usually 
stimulated at selected locations while fixating on a central fixation cross. Mapping the angle component of the retinotopic map reveals multiple horizontal and vertical meridian representations arranged in approximately parallel bands along the cortical surface (Figure 13). These vertical and horizontal meridian representations alternate and define the borders between mirror-symmetric retinotopic areas.

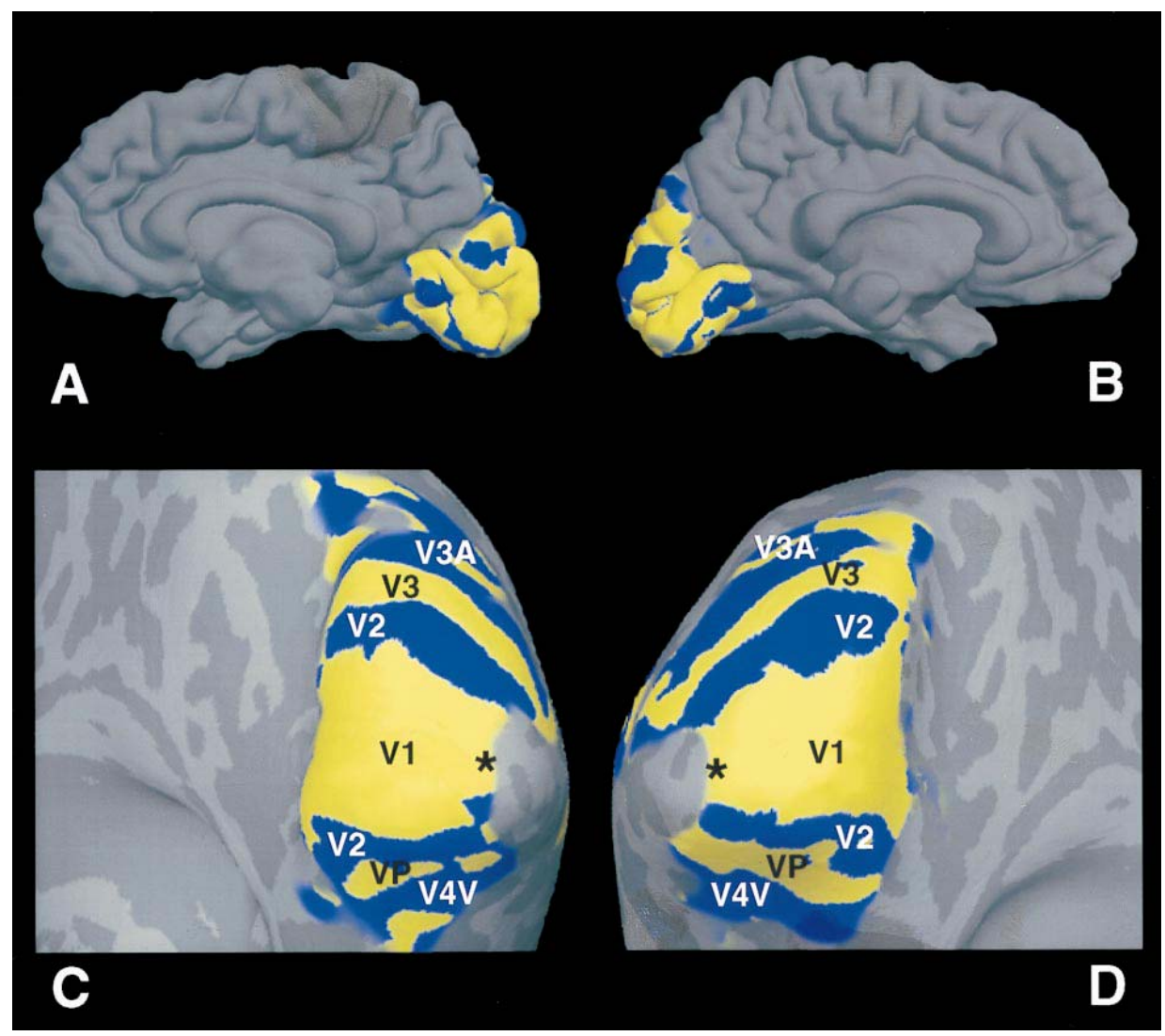

\section{Figure 13}

Topography of primary visual cortex and surrounding areas. ( $A$ and $B$ ) Field sign analysis of retinotopic cortical visual areas from right and left hemispheres (respectively) in a single subject. Both hemispheres are views of the medial bank, in its normal, folded configuration. Thus in $A$, anterior is to the left, and posterior to the right. In $B$, this is reversed. The field sign maps are based on two scans measuring polar angle (rotating thin ray stimulus), and two scans measuring eccentricity (expanding thin ring stimulus). $(C$ and $D$ ) Same data, in a cortically "inflated" format, now viewed from a more posterior-inferior vantage point. Again the left panel shows the right hemisphere, and the right panel shows the left hemisphere from the same subject. Human retinotopic areas revealed by the field sign analysis have been labelled (V1, V2, V3, VP, V3A, $\mathrm{V} 4 \mathrm{v}$ ). Cortical areas with a visual field sign (polarity) similar to that in the actual visual field are coded blue, and those areas showing a mirror-reversed field polarity are coded yellow. Also labelled is the foveal representation in V1 (black asterisks). Gyri and sulci in the folded state (e.g., $A$ and $B$ ) are coded in lighter and darker shades of gray (respectively) in the inflated format ( $C$ and $D)$. In this subject, area V1 is somewhat larger than normal, extending well past the lips of the calcarine fissure. However, as in most subjects, the V1 representation of the extrafoveal horizontal meridian lies near the fundus of the calcarine fissure (from Tootell et al., 1998). 
Perpendicular to these bands lie iso-eccentricity bands, which constitute an eccentricity gradient (Figure 14). As mentioned earlier, the representation of the fovea is greatly expanded compared to the representation of the periphery.

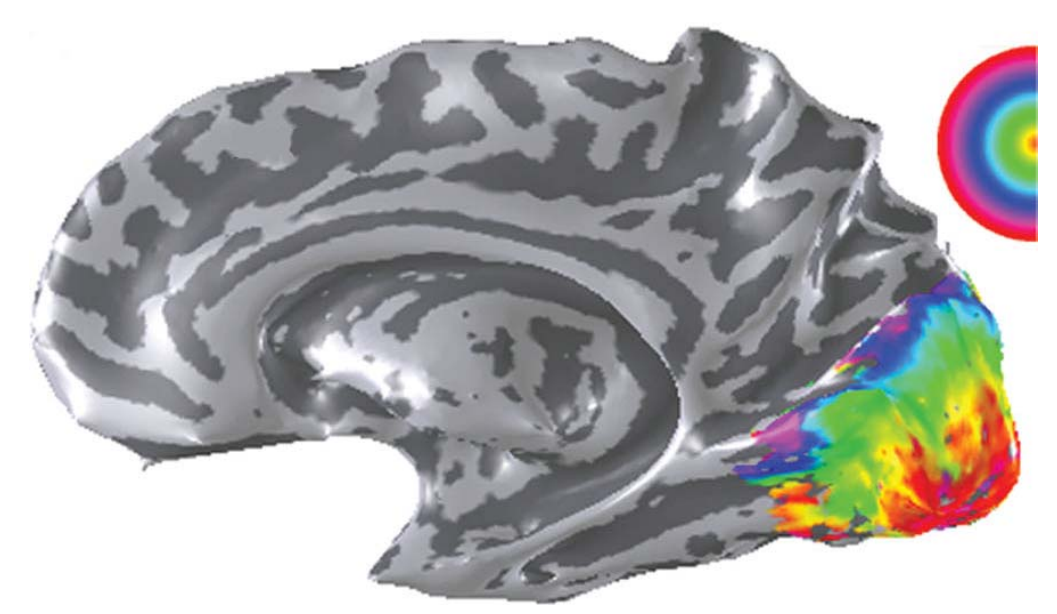

Figure 14

The eccentricity map was measured by using expanding rings comprising contrast-reversing dartboard patterns. The eccentricity map was projected on the right hemisphere of an inflated brain. The colour overlay indicates the eccentricity that produces the most powerful response at each cortical location (see the coloured legends on the right) (from Wandell et al., 2005).

A large-scale summary of known visual areas illustrated on the unfolded surface of one hemisphere is shown in Figure 15. The cortex is unfolded by cutting along the calcarine sulcus, thereby splitting the horizontal meridian of area V1. The flat map reveals an intriguing pattern about the relationship between hierarchical and specialized processing: The two organizing principles, hierarchical processing and functional specialization, are neatly translated into two orthogonal axes laid upon the unfolded human visual cortex (Grill-Spector \& Malach, 2004).

The hierarchical progression is arranged along the back-to-front axis, whereas the functional specialization axis is situated orthogonally to the hierarchy axis along the dorsoventral direction. The hierarchy axis starts from lower area V1 posteriorly and ascends in retinotopic cortex going through areas V2, V3 and V3a dorsally and V2, V3/VP, and V4 ventrally, finally reaching into the object-related areas. The hierarchical structure of the visual system is characterized by two properties: increasing receptive-field size and increasing receptive-field complexity. An increase in receptive-field size is associated with an increase of spatial integration of information by the neurons and a corresponding loss of retinotopic organization. Early visual areas such as LGN and V1 respond primarily to simple visual features such as oriented edges within very small receptive-fields, whereas 
anatomically later regions, including inferotemporal cortex (IT), posterior parietal cortex (PPC), and frontal eye fields (FEF), respond to more complex and abstract stimulus properties within receptive-fields that encompass large expanses of the visual field.

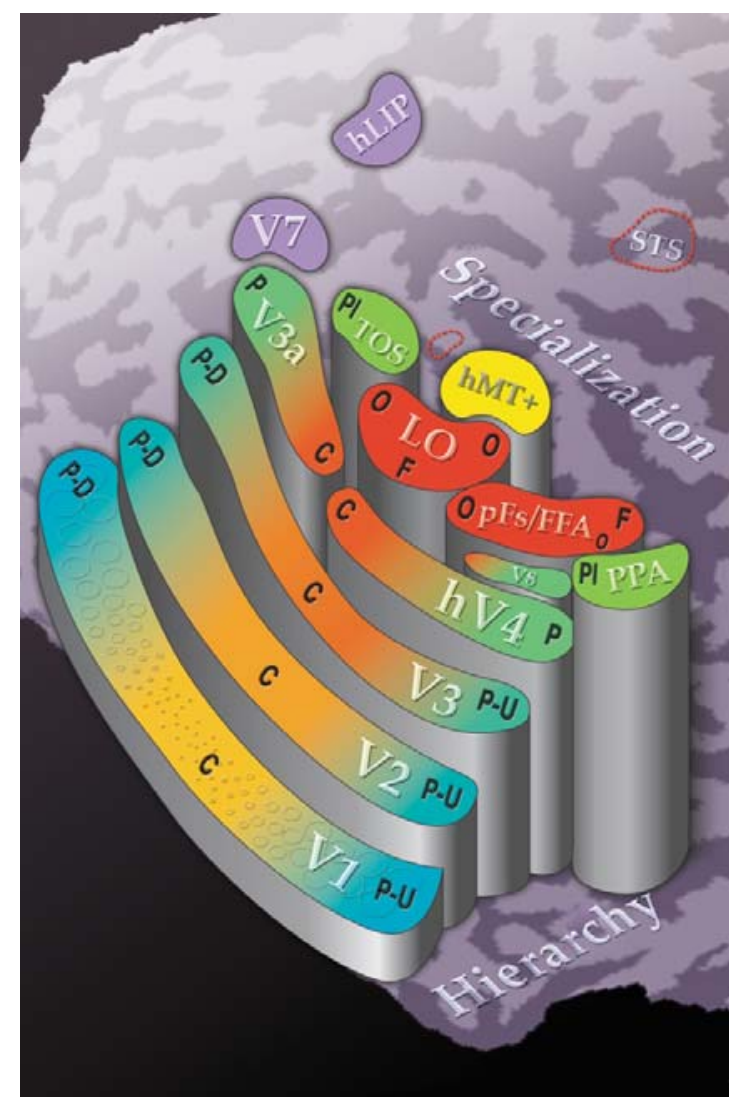

\section{Figure 15}

Schematic atlas of human visual cortex. The schematic layout of human visual areas is presented on an unfolded right hemisphere, illustrating the orthogonal axes of hierarchy and specialization (labelled along the axis direction). The visual areas are arranged in a staircase fashion to illustrate the hierarchical sequence of increased abstraction leading from primary visual cortex to high-order visual areas. The orthogonal, specialization axis is illustrated through the colour scale. The specialization is manifested in early cortex as a transition from central $(C)$ to peripheral $(P)$ visualfield representations, associated with high and low magnification factors, respectively. In higherlevel cortex, the specialization is manifested as a transition from regions that respond preferentially to objects/faces $(O, F)$, and are related to central-biased, high-magnification representations, to regions that respond more strongly to places, buildings, and scenes $(B)$ and are related to peripheral-biased, lower-magnification representations. B, buildings/scenes; C, central; P-U, P-D, peripheral representation of upper and lower visual fields, respectively; Pl-, place; F-, faces; O-, objects (from Grill-Spector \& Malach, 2004).

Along the specialization axis the visual areas can be examined under the global framework of eccentricity. In early visual cortex (V1-V3) eccentricity begins with regions specializing in peripheral vision (peripheral lower-field representation), travels through regions specializing in central vision, at the centre of the visual system, and continues ventrally 
into peripheral representations (peripheral upper-field representation). More anterior visual areas (V3a, V4) transverse the periphery-centre-periphery specialization sequence, but loose the separate upper versus lower visual field representations. Finally in high-level, object-selective cortex, a sequence of object-category specializations can be found, which roughly parallels the periphery-centre-periphery sequence. Regions that respond to places (transverse occipital sulcus (TOS) or parahippocampal place area (PPA)) are associated with peripheral representations, whereas regions that respond more strongly to faces (fusiform face area, FFA) are associated with central representations (Hasson et al., 2002; 2003; Levy et al., 2001; Malach et al., 2002). The centre/periphery map provides a global organization principle of the entire visual cortex, cutting across the borders between cortical areas.

Studies in macaque monkey have shown that the visual cortex contains a mosaic of several dozen visual areas that collectively occupy a large fraction of cerebral cortex (approximately $50 \%$ in the macaque and $25 \%$ in humans) (Felleman \& Van Essen, 1991; Van Essen, 2005). These areas are richly interconnected by hundreds of reciprocal corticocortical pathways that underlie an anatomically based hierarchy containing multiple processing streams (Van Essen, 1995). Furthermore, there exists a complex pattern of reciprocal connections with the pulvinar, a nucleus buried within the thalamus (Van Essen, 2005). Information flow through these corticocortical and corticothalamic circuits is regulated very dynamically by top-down and bottom-up processes (including directed visual attention).

\section{Figure 16}

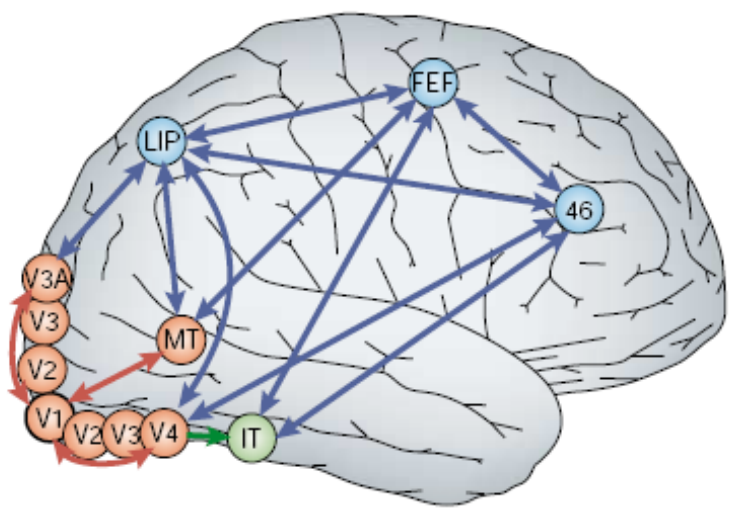

Connections between a subset of cortical visual areas (schematic diagram). Primary visual cortex (V1) has direct reciprocal connections with posterior extrastriate areas V2, V3, V3A, V4 and MT (red). (Not all backprojections to V1 are shown; extensive reciprocal connections between extrastriate areas are omitted.) Some of these posterior extrastriate areas have reciprocal connections with area LIP (lateral intraparietal), the frontal eye fields (FEF) and area 46 (blue), which are involved in visual attention and motor planning. According to some hierarchical models, 
only extrastriate areas such as MT, V4 and inferotemporal cortex, which project directly to frontalparietal areas that are involved in attention or motor planning, can contribute directly to visual awareness. By contrast, interactive models propose that recurrent loops between $\mathrm{V} 1$ and posterior extrastriate areas (such as MT and V4) are essential for maintaining a visual representation in awareness (from Tong, 2003).

Within this widespread network of visual areas, V1 plays a crucial role as the primary distributor of almost all visual information that reaches the other cortical areas. The LGN is the thalamic station in the retinocortical projection stream and has traditionally been viewed as the gateway to the visual cortex. About $90 \%$ of retinal fibres project through LGN to V1. The remaining $10 \%$ project to various subcortical structures, and include a pathway from the retina through the superior colliculus to the pulvinar, which has reciprocal connections with several extrastriate areas. From V1, information is transferred to various extrastriate visual areas for further analysis, including areas V2, V3, V3A, V4 and V5/MT (Figure 16) (e.g., Salin \& Bullier, 1995). These extrastriate areas receive almost all of their inputs, either directly or indirectly, from V1. Areas such as V4 and V5/MT project directly to visual areas in the parietal and frontal lobes that are implicated in attention, working memory and motor planning. Most connections between visual areas consist of both feedforward and feedback connections, pointing towards a high degree of interactive processing (Tong, 2003).

\subsection{Space-based theories of attention}

Selective attention is a broad term that refers to a variety of different behavioural phenomena. Research in the $60 \mathrm{~s}$ and $70 \mathrm{~s}$ of the $20^{\text {th }}$ century has mainly led to three different concepts describing selective visual attention either as space-based (spatial; directing attention to a specific location in the visual field), object-based (directing attention to an object) or feature-based (directing attention to particular visual features, such as colour, orientation or direction of motion) attention (for review see Johnston \& Dark, 1986; Driver, 2001). As this thesis concentrates on spatial attention, the other concepts of selective attention are not further discussed.

Space-based theories assume that the spatial location plays a unique role in the selection of information. Attentional mechanisms are needed to select relevant, and to filter out irrelevant information from cluttered visual scenes. Therefore, attention can be directed to specific parts of the visual field, where information processing is facilitated in contrast to unattended locations (Posner, 1980; Driver \& Baylis, 1989; Slotnick et al., 2003). Spatial 
attention has been shown to improve the accuracy and speed of subjects' responses to target stimuli that occur at the attended location (Posner, 1980). Attention also increases the perceptual sensitivity for the discrimination of target stimuli (Lu \& Dosher, 1998), increases contrast sensitivity (Cameron et al., 2002), reduces the interference caused by distracters (Shiu \& Pashler, 1995) and improves acuity (Yeshurun \& Carrasco, 1998).

Such transient spatial attention effects have been described in metaphors such as a 'spotlight' (Eriksen \& Hoffman, 1973; Posner et al., 1980; Treisman \& Gormican, 1988), a 'searchlight' (Crick, 1984), or a 'window' (Connor et al., 1997) of attention. Recently, the study of visual spatial attention has foremost been dominated by 'spotlight theories', claiming that selective attention can be compared to a spotlight illuminating anything located within the region covered by its beam (James, 1950). Several theories have been proposed using the idea of a spotlight as a metaphor to account for the distribution of attentional resources in visual space: 'single spotlight', 'zoom lens', and 'multiple spotlights' model.

\subsubsection{Single spotlight of attention}

Visual attention as a single spotlight is directed to a unitary contiguous region of visual space, where stimuli will be processed preferentially, and must be redirected whenever detailed information from a different part of the visual field is to be processed (Posner et al., 1980; Duncan, 1984; LaBerge, 1983). Originally the beam of the attentional spotlight was thought to be a fixed size of approximately $1^{\circ}$ of visual angle (Eriksen \& Eriksen, 1974), but further work by Eriksen and colleagues (Eriksen \& Yeh, 1985) showed that the spotlight diameter could vary in size and shape (Posner et al., 1980). The size of the attentional spotlight can be adjusted to the spatial arrangement of the attended stimuli and to current task demands (LaBerge, 1983; Eriksen \& St. James, 1986; Pan \& Eriksen, 1993). Furthermore, it has been suggested that there might be a cost associated with spreading the spotlight over a larger area (Castiello \& Umiltà, 1990). Stimuli falling inside the beam of attention are detected faster and more accurately than stimuli outside the attentional beam (Posner et al., 1980; Posner \& Petersen, 1990; Luck \& Hillyard, 1995; Van der Heijden et al., 1987).

Although the unitary nature of the beam is traditionally considered to be the fundamental property of the moving-spotlight model, the manner in which the beam moves through the visual field has yet to be resolved. Initially, the beam was thought to move in an analogue 
manner across the visual field, such that locations on the way to the cue would temporally become attended to as the beam passed over (Shulman et al., 1979). Adopting the analogue movement of the spotlight, Tsal (1983) estimated a constant attentional velocity of $1 \% \mathrm{~ms}$ and speculated that little information is collected as the beam of attention is moving. Later it was proposed that attentional movement is time invariant, and that the attentional mechanism adjusts its velocity in proportion to the distance to be travelled, resulting in a nearly constant travel time for a wide range of different distances (Remington \& Pierce, 1984; Kwak et al., 1991). There is also evidence that the beam of attention essentially "turns off" during movement, creating the appearance of a discrete switching of attention from location to location (Briand \& Klein, 1987). Discrete properties of a shift of visual spatial attention have been proposed as well (Chastain, 1992a; 1992b; Sperling \& Weichselgartner, 1995).

Since the spotlight of attention as well as overt eye movements can both be summoned by a peripheral event (e.g. exogenous cue) and both can be directed voluntarily to a location in visual space (e.g. endogenous cue) a close functional (Posner, 1980) and physiological (Wurtz \& Mohler, 1976) relationship between these systems has been proposed. In everyday life, a shift of attention is usually followed by an overt eye movement indicating that eye fixation and attentional locus are usually highly correlated. Following the inferences of the spotlight metaphor, the locus of directed attention in visual space is thought of as having greater illumination than the areas to which attention is not directed, or areas from which attention has been removed. However, the point of fixation does not necessesarily represent the location from which information is processed, indicating an independence between the line of sight and visual selection. In the laboratory, spatial attention effects can be studied in a sustained manner by having subjects fixate a specified point but direct and maintain their attention on a point specified elsewhere. This 'covert attention' paradigm has revealed a great deal about visual spatial attention in experiments including psychophysics, neuroimaging, and cortical electrophysiology. It has been demonstrated that when a saccade is made toward a particular location, attention moves to that location before the onset of the saccade (Posner \& Cohen, 1984). Thus, it has been shown that movements of attention can occur without making eye movements (Eriksen \& Hoffman, 1972; Posner et al., 1980). As Broadbent (1982) stated that eye movements may reinforce selectivity, yet it can occur without them.

One important feature of the single spotlight model is that focal attention cannot be assigned to non-contiguous locations in the visual field without processing information in 
all intermediate positions. That means that the metaphorical beam cannot be split. Using a modification of the basic cueing paradigm in which two different potential target locations were cued, Posner and colleagues (1980) investigated whether attention could be allocated to different locations of the visual field. Each trial had a primary cue that was valid on $65 \%$ of the trials and a secondary cue that was valid on $25 \%$. Response time facilitation for the targets was compared revealing a benefit for the secondary cue's locations only when it was adjacent to the primary cue. This was regarded as evidence for a single attentional focus. However, the design of this study may not have met some important preconditions for splitting attention into non-contiguous regions, such as a high incentive to process both cues. The secondary prime was valid only in a quarter of the trials. Thus, there was only a marginal motivation to attend to the secondary cue. Further studies, in which evidence supported unitary attention also employed cues with a validity of less than 50\% (Eriksen \& Yeh, 1985; McCormick \& Klein, 1990). In line with the single spotlight theory Heinze and colleagues (1994) demonstrated an attentional enhancement of ERP component P1 (positive potential occuring $100 \mathrm{~ms}$ post-stimulus) for probes appearing at two locations occupied by targets to be compared, but also for probes at the location in between the two targets. However, it was pointed out that the P1 component is an indirect measure and may not reflect all aspects of spatial attention.

The physiological correlate of the spotlight of attention has been investigated by measuring the neuronal and haemodynamic response in foveal and/or peripheral visual field representations in striate and extrastriate visual cortex. Both neuroimaging and singleneuron studies provided evidence that selective visual attention can modulate responses in visual cortex (Corbetta et al., 1991, 1993; Motter, 1994; Treue \& Maunsell, 1996; Beauchamp et al., 1997; O’Craven et al., 1997; Kastner et al., 1998; McAdams \& Maunsell, 1999; Somers, 1999; Tootell et al., 1998; Watanabe et al., 1998; Gandhi et al., 1999).

Using fMRI Tootell and colleagues (1998) investigated the neuronal correlate of the spotlight of attention by mapping spatial attention in direct comparison to the cortical retinotopy. A covert attention paradigm was used to determine the effects of spatially selective attention to four different locations and spatial attention compared to passive viewing. The results showed that attention to a specific location in the visual field produced higher activity in the sensory representation of those same locations. Furthermore, the hierarchical model of spatial attention processing, which predicts higher attentional activity in extrastriate cortical areas compared to striate cortex because of the 
size of the receptive fields, could be confirmed. Selective attention was absent in V1 which could be related to the small size of the stimuli. In line with these results, numerous studies found that attention modulates neuronal activity in extrastriate visual areas such as V2, V4 and V5/MT (Moran \& Desimone, 1985; Corbetta et al., 1990; Corbetta et al., 1991; Beauchamp et al., 1997; Spitzer et al., 1988; Luck et al., 1997; Treue \& Maunsell, 1996; Connor et al., 1997; Motter, 1993) whereas little or no attentional modulation was observed in V1 (Moran \& Desimone, 1985; Luck et al., 1997; Luck \& Ford, 1998; O’Craven et al., 1997; Heinze et al., 1994). However, in the last decade many studies provided evidence for robust effects of attention in primary visual cortex as well. The engagement of striate and extrastriate visual areas in spatial selective attention has been demonstrated during attentionally demanding visual discriminations tasks (Somers et al., 1999; Martinez et al., 1999; Gandhi et al., 1999) as well as during simple visual tasks (Watanabe et al., 1998). For example, Brefczynski and De Yoe (1999) used a task in which the subject's gaze remained fixated in the centre of the visual field while attention was directed to a cued location within an array of segments. The subjects' task was to detect specific colour/orientation conjunctions within the cued segment while ignoring other uncued segments. Results showed a precise match of the topography between the cortical representations of the attended target presented in isolation and purely attention-driven activity in primary visual cortex, in dorsomedial and ventral occipital visual areas (Figure $17)$. 


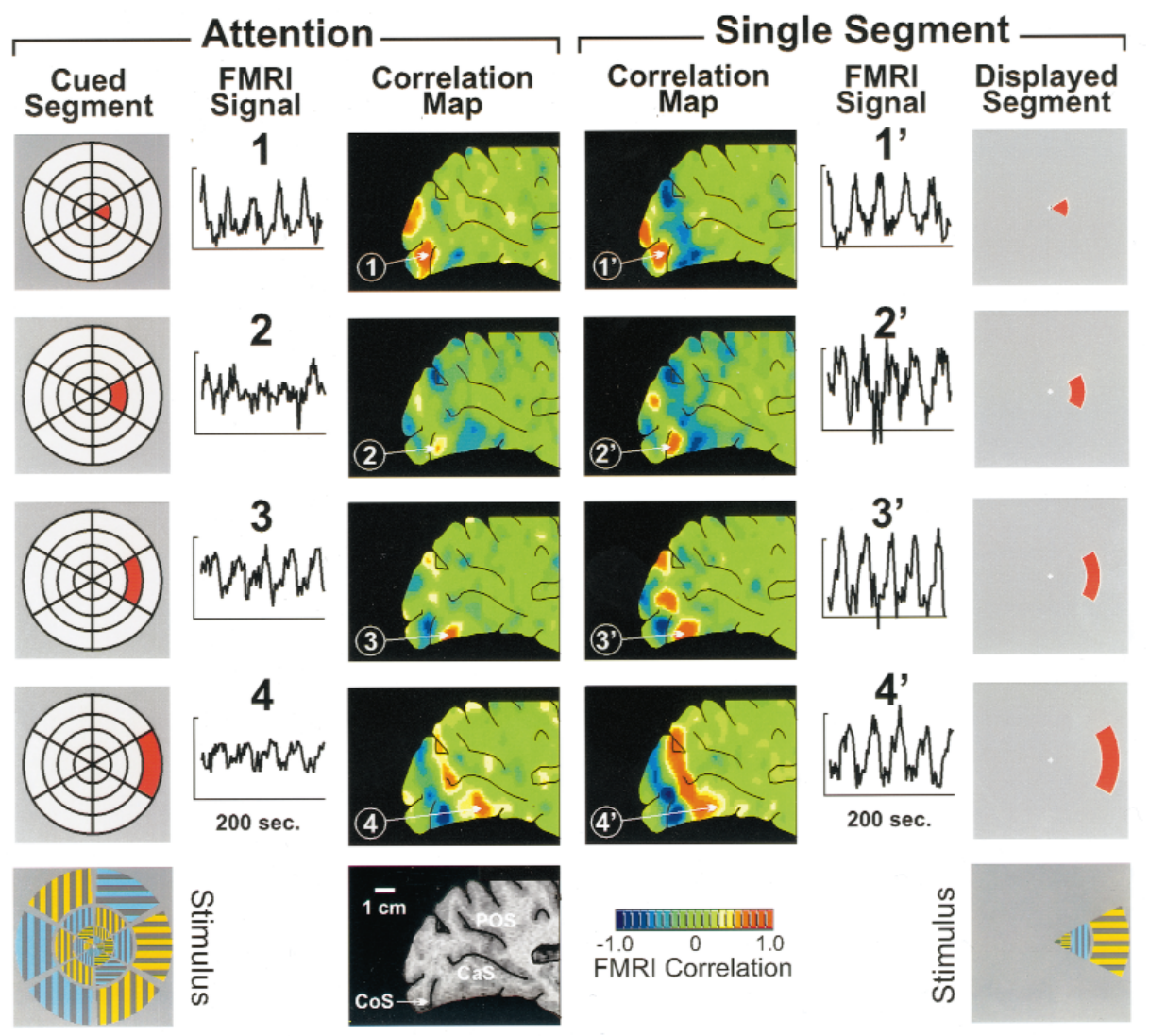

\section{Figure 17}

Retinotopic attentional modulation compared to activation evoked by cued targets alone. Cued segment (left column) shows schematic sequence of target segments cued for attentional scrutiny. The stimulus (bottom left) consisted of blue and orange line segments arranged into sectors radiating out from the central fixation point. FMRI signal (left) shows modulation of individual voxels at sites indicated on adjacent correlation maps. Temporal phase shift of the signal at each site identifies the corresponding locus of attention. Correlation maps show sites where timing of modulation was positively correlated (red) or anti-correlated (blue) with the timing of attentional shifts. Displayed segment (right column) shows schematic sequence of single segments presented during otherwise identical control experiment. Composite of single segments shown in stimulus bottom right. FMRI signal and correlation map on right show results of control experiment. Structural MRI (bottom) is a parasagittal section (13.6 mm left of midline) through occipital lobe in same plane as correlation maps. Sulcal landmarks; CaS, calcarine sulcus; CoS, collateral sulcus; POS; parieto-occipital sulcus (from Brefczynski \& De Yoe, 1999).

In summary, the studies discussed above clearly show that the extent of the physiological effects of attention observed in different cortical areas depend not only on the stimulus, but also on the nature of the behavioural task and the strength of the psychophysical effects. However, it has been demonstrated that when the focus of attention shifts, the cortical enhancement shifts in precise correspondence, indicating that the observed physiological correlate of spatial attention is accurately described using a retinotopic metric. Furthermore, it has been shown that attentional modulation occurs at a variety of cortical sites, even at the primary visual cortex. 


\subsubsection{Zoom lens model}

The zoom lens model (Eriksen \& St. James, 1986; Eriksen \& Yeh, 1985) is a variant of the spotlight metaphor of attention and suggests a focus of variable width, similar to a zoom lens which improves the adaptability of the system. The most important difference between these two metaphors is that the zoom lens model makes explicit predictions about an inverse relationship between size of the attentional focus and processing efficiency bringing the concept of limited resources into structural models of attention. As visual attention is a limited-resource system, increasing the size of the attended region reduces the attentional benefit at any particular location within the attended region because all available resources are spread over a great area. When reliable spatial information is provided, the system switches to its focused mode, concentrating all resources on a circumscribed area, leaving little or no resources for simultaneous processing of other locations. When it is not necessary to focus attention on a specific location attention is distributed uniformly over the visual field. Thus, attention can either be allocated to a small region of visual space with high resolving power, or alternatively directed to a large region at a reduced resolution. The change of locus of attention would seem to be discrete, with the time required for shifts from one location to another being independent of the distance between the locations (Eriksen \& Webb, 1989; Murphy \& Eriksen, 1987). From this resource-based conception of attention, attentional allocation consists in the gradual but rapid buildup of resources at the attended location. To sum up, the zoom lens model has three main features (Eriksen \& Murphy, 1987; Eriksen \& St. James, 1986; Eriksen \& Yeh, 1985): (1) the size of the attentional focus is variable, (2) processing efficiency is inversely related to the size of the attended area, and (3) the rate of accumulation of resources in a specific position is independent of the distance between that position and the position where they were previously concentrated.

Indeed the predictions of the zoom lens model could be confirmed by behavioural studies. Egeth (1977) demonstrated that the size of the attentional focus can vary and found a concomitant reciprocal variation in processing speed. Beck and Ambler (1973) provided similar results in showing that discrimination accuracy was higher when attention was focused than when it was distributed. LaBerge (1983) investigated the spatial extent of focused attention and found that the size of the attentional focus can vary according to task demands. In accordance with the zoom lens model, Eriksen and St. James (1986) showed that reaction times to targets decreased as the size of the attended area increased indicating 
an inverse relationship between size of attentional focus and processing efficiency. Additionally, the results suggested that the edge of attentional focus was not sharply demarked, but showed a gradual dropoff. In later work, Castiello and Umiltà (1990) readdressed the issue concerning the features of the attentional focus and could verify previous findings concerning the adjustment of the the attentional focus associated with different task demands, the decrease in processing efficiency in relation to the increase of the attended area and the gradual dropoff in processing efficiency around the attentional focus.

Müller and colleagues (2003a) sought physiological evidence for the zoom lens model by using fMRI. The aim of the study was to detect whether the extent of activated visual cortex increases with the size of the attentional focus and the level of activation is related to the discrimination performance for the target stimuli. Subjects had to identify circles of a certain colour at different locations which were precued and determined the size of the attended region. The behavioural observation that reaction times increased and accuracy dropped with increasing size of the attended region was reflected in the level of activation in striate and extrastriate visual areas. As the size of the attended region increased, the extent of activated retinotopic visual cortex increased as well while the level of neuronal activity decreased in a given subregion representing a certain location in the visual field. Moreover, additional activation in subregions was observed when attention had to cover a large region (Figure 18). The authors proposed that based on the idea that neuronal activity in visual areas reflects their processing capabilities, the inverse correlation of neuronal activity with processing speed and accuracy can be taken as physiological evidence of the zoom lens model. In line with these results, a recent fMRI study by McMains and Somers (2005) provided similar findings. An increase of the size of the attentional focus is associated with a decrease of processing efficiency and activity in primary and secondary visual areas. However, an uniform spread of attention across the attended regions could not be found as proposed by the zoom lens model, rather a landscape of peaks and valleys of attentional modulation across the cortical representation of the visual field was observed. 


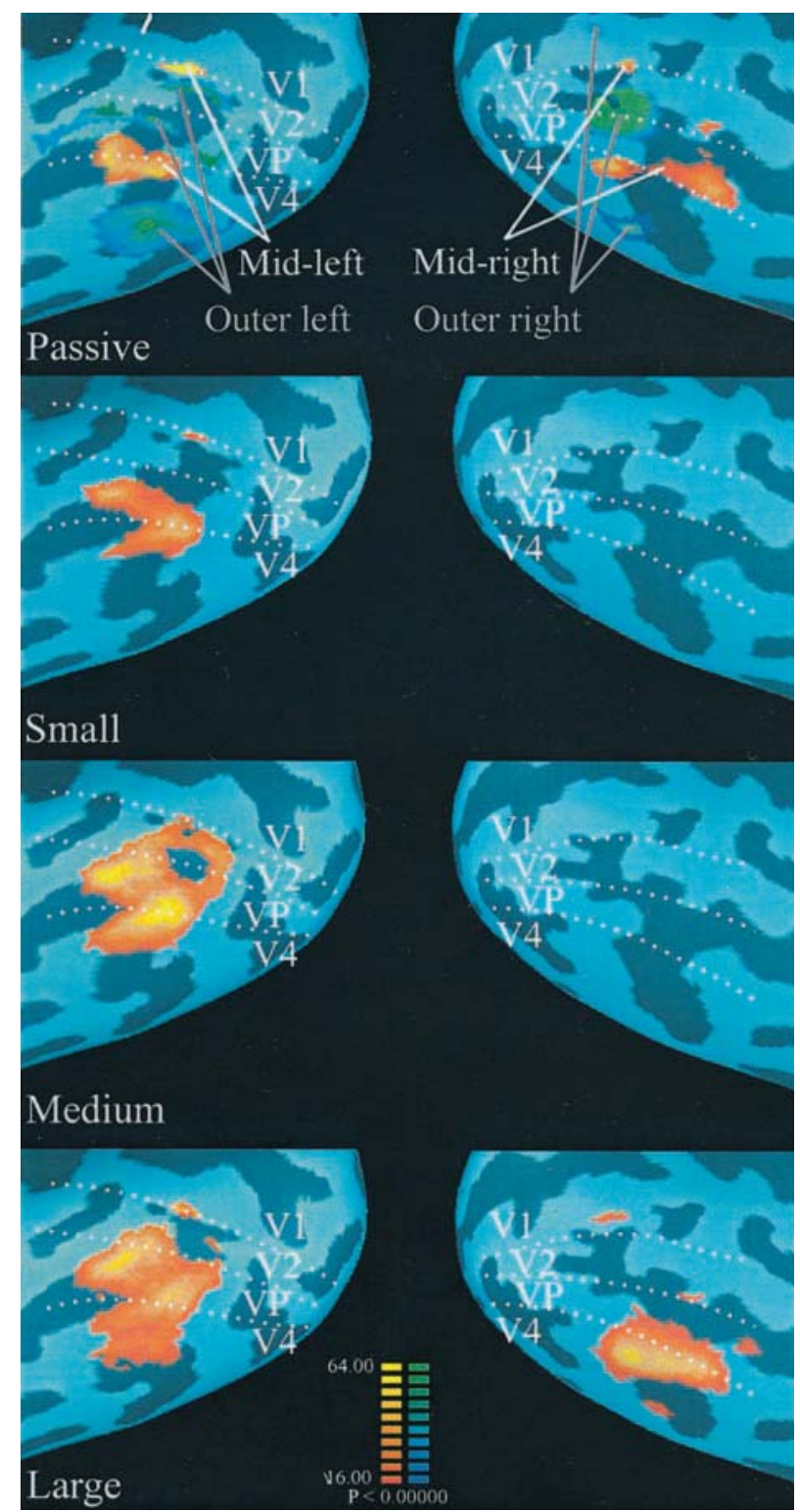

\section{Figure 18}

First row: Activation maps elicited by passive viewing of stimuli at the four single locations during ROI mapping. Next rows: Activity after cue onset in the attention task, when either only the middle left location (small, second row), both left locations (medium, third row), or all locations (large, fourth row) were cued. Data are from one subject. Left hemisphere on the right, viewed from medial and below. Note that because the middle positions were close to the vertical meridian, the voxel cluster seem to cross the borders of visual areas V1/V2 and VP/V4 (from Müller et al., 2003a). 


\subsubsection{Multiple spotlights of attention}

Like the gradient model of attention the multiple spotlights theory allows the simultaneous selection of multiple and spatially distinct regions of space, while information in intermediate regions is excluded: The 'beam' of the attentional spotlight can be divided (Shaw \& Shaw, 1977; Awh \& Pashler, 2000). Furthermore, it has been argued that the ability to divide the attentional spotlight in the presence of two distinct behaviourally relevant locations benefits resource conservation compared to the zoom lens model, where a single spotlight would include task-irrelevant regions between the two relevant locations (McMains \& Somers, 2005). Because of this processing inefficiency, the distance between two attended regions might influence the selection of the attentional mechanism.

In support of this theory, several studies found that attention can be divided across noncontiguous locations (Kramer \& Hahn, 1995; Awh \& Pashler, 2000; Hahn \& Kramer, 1998; Castiello \& Umiltà, 1992; Bichot et al., 1999; Eimer, 2000; Schmidt et al., 1998) and that visual attentional selection can be precise enough to exclude irrelevant stimuli if there is a minimal spatial separation between the attended and the competing stimuli (Miller, 1991; Yantis \& Johnston, 1990). Early studies (Shaw \& Shaw, 1977; Shaw; 1978) presented accuracy and reaction time data, respectively, suggesting the possibility that subjects simultaneously allocate different amounts of attention to different locations in the visual field in order to optimize performance.

Kramer and Hahn (1995) sought to resolve the discrepancy between studies supporting a single attentional spotlight and those providing evidence for a split of attention and proposed an explanation concerning this issue. Some studies may have failed to obtain a split of attention because the stimuli in these studies were presented as sudden onsets, which in some circumstances will automatically capture attention (Theeuwes, 1995; Yantis, 1995). They suggested that the attentional capture of sudden-onset distracter stimuli may make it difficult or even impossible for subjects to maintain the attentional focus on previously cued locations. To test this hypothesis, subjects performed a task in which they had to perform a same/different matching task on two target letters while ignoring intervening distracter letters. The positions of the target letters were precued with two boxes which were located in different hemifields on an imaginary circle. Letters were either presented as sudden onset or nononset stimuli (when premasks were changed into the targets and distracters). As predicted, distracter letters interfered with performance in the sudden-onset condition, but not in the nononset condition, leading Kramer and Hahn to 
the conclusion that subjects were capable to attend simultaneously to the two cued nonadjacent locations. Further behavioural experiments by Hahn and Kramer (1998) confirmed the previous results in showing that subjects can split their attentional focus. Moreover, it has been demonstrated that the flexibility of spatial attention in form of the division of attention among non-contiguous locations is independent of the boundaries of the hemifields: Subjects were able to concurrently attend to different locations within as well as between hemifields. In line with these results, Schmidt and colleagues (1998) used illusory line motion as an index of attention to show that attention can be deployed to multiple locations at the same time. Bichot and colleagues (1999) used several variations on a monitoring task to support the notion of separate attention windows that open and close independently. Furthermore, Awh and Pashler (2000) provided evidence for multiple foci of attention in the visual field and for a bimodal distribution of processing quality, by demonstrating that accuracy was highest at two non-contiguous locations and markedly lower in between. In a recent study (Scharlau, 2004) the spatial distribution of attention was assessed by perceptual latency priming: the latency benefit of an attended visual stimulus, as compared with an unattended stimulus. The experiments are in accordance with split attentional foci in reporting attentional priming effects at two non-contiguous locations with little or no priming at the intervening location.

Electrophysiology was used to characterize the temporal features of divided attention (Eimer, 2000). Subjects were instructed to focus attention on ring-shaped regions of visual space to detect infrequently presented targets at a given eccentricity. Attention did not affect sensory-evoked ERP components like P1 and N1 amplitudes (positive or negative potential occurring $100 \mathrm{~ms}$ post-stimulus), suggesting that behavioural effects observed in such complex spatial selection tasks are likely to result from post-perceptual attentional processes rather than attentional modulations of sensory stages. Beyond $200 \mathrm{~ms}$ poststimulus, differences in ERPs elicited by stimuli at attended and unattended eccentricities indicated some flexibility in distribution that is inconsistent with a zoom lens of attention. Müller and colleagues (2003b) used an electrophysiological measure of attentional allocation (the steady-state visual evoked potential, SSVEP) to show that the spotlight may be divided between spatially separated locations over more extended time periods. Therefore, stimuli were presented concurrently at attended and interposed unattended locations during the recording of SSVEPs, which are the electrophysiological response of the visual cortex to the rapidly repeating (flickering) stimuli. The maximum amplitudes of the recorded SSVEPs at each frequency were observed at occipito-temporal scalp sites 
contralateral to the visual field of stimulation. In intermediate ignored positions the SSVEP was reduced in relation to when the same position was attended. These SSVEP recordings provide evidence that the spotlight of spatial attention can be divided to facilitate processing of stimuli in spatially non-contiguous locations over periods of several seconds, which extends the findings of split attentional foci for brief exposures by Hahn and Kramer (1998) and Awh and Pashler (2000). The possibility that subjects were rapidly switching attention between the separated locations to achieve accurate performance instead of dividing attention between locations could be ruled out through the use of brief $(181 \mathrm{~ms})$ target durations. There is a general agreement that the minimum time needed to identify a target at one location and switch attention to identify a target at a second location is in the range of 200-500 ms. The findings of Müller et al. (2003b) provided evidence that the spotlight might be divided over sustained periods of time, thereby facilitating inputs from separated locations at the level of extrastriate occipital pathways. The authors concluded that this would appear to be a highly efficient early selection mechanism for distributing attention optimally to dispersed stimuli in the visual surroundings. In a recent follow-up study by Malinowski and colleagues (2007) previous findings could be extended by demonstrating that a split of attention is even possible within one hemifield. However, task performance was lower when attention was divided in the same hemifield compared to a split of attention across hemifields.

The question of whether the attentional spotlight can be split has been addressed in only a few functional imaging studies so far. Only one group using fMRI has documented attentional modulation in striate and extrastriate visual areas for two separately attended locations with a lack of enhanced activity at the irrelevant intermediate regions (McMains $\&$ Somers, 2004; 2005). Subjects viewed five simultaneously displayed streams of letters and digits in rapid serial visual presentation (RSVP) and fixated on a RSVP stream that was placed in the middle of the screen and was used as an important distracter location. The remaining four RSVP streams were placed equidistant from the fovea, one in each of the four visual quadrants. The experiment consisted of two tasks: In the Attend1 task subjects were trained to monitor only one RSVP stream for the appearance of a digit whereas in the Attend2 task participants had to detect digits among two letter streams and report if the digits matched or mismatched. The retinotopic locations of the five RSVP streams on the visual cortex were defined as regions of interest (ROIs) in separate ("localizer") fMRI scans. Results are consistent with the multiple spotlight hypothesis and demonstrate increased activation for Attend 2 versus Attend1 in the defined ROIs 
corresponding to the attended RSVP streams while sparing the fovea (Figure 19). In a control study McMains and Somers showed that the foveal region could be selected as well and modulate attention, when it is relevant for the task. This supports the view that the sparing of the foveal region in the other experiment occurred because subjects split their attentional spotlights to ignore the foveal region and to attend to the two peripheral quadrants. Attentional modulation was observed in all cortical areas contained within the ROIs (V1, V2, V3/VP, V3A, V4v, V8). The specific definition of ROIs in the primary visual cortex (V1 and V2) for the four RSVP streams revealed that multiple spotlights of attention can occur in early retinotopic visual cortex, including area V1.

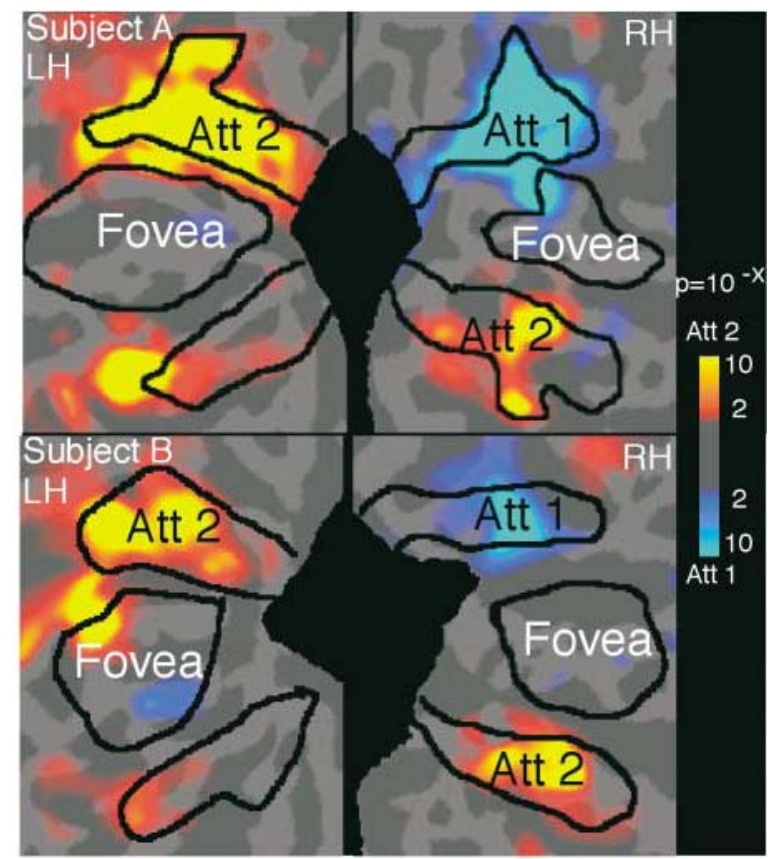

\section{Figure 19}

Contrast between the Attend2 (yellow/red) versus the Attend1 (blue) task of two subjects. Black outlines represent regions of interest. Subjects show an area of activation corresponding to the Attend1 stimulus in the right hemisphere above the calcarine sulcus. For Attend2, there are two main areas of activation corresponding to the Attend2 locations in the left and right hemisphere with sparing of intermediate regions (fovea) indicating a split of the attentional spotlight (from McMains \& Somers, 2004).

Another question which was investigated in an additional experiment by McMains and Somers (2004) addressed the issue of splitting the spotlight within a hemisphere and visual quadrant. The attention-tasks remained the same as in the prior experiment, but the RSVP streams were presented in only one hemifield. The fMRI results again revealed attentional enhancement of early visual areas, including V1 and V2, for the two attended locations and significantly less attentional enhancement in the intervening region. These findings are 
inconsistent with the zoom lens hypothesis, which claims that attention should be strongest at the intermediate location, and demonstrate that multiple spotlights of spatial attention can occur within a quadrant of the visual field. But it is important to note that at least some attentional activation also occurred at the distracter location which may indicate that the resolution of multifocal spatial attention is limited and it may be easier to split attention across the hemispheres than to split within a hemisphere. Recently, it has been argued that the ability to divide the attentional spotlight in the presence of two distinct behaviourally relevant locations benefits resource conservation compared to the zoom lens model, where a single spotlight would include task-irrelevant regions between the two relevant locations (McMains \& Somers, 2005). According to the zoom lens model, processing efficiency decreases as the size of the attended region increases. Therefore, the distance between two attended regions might influence the selection of the attentional mechanism to ensure maximized processing efficiency. To test this hypothesis different experimental runs were performed where attention had to either be allocated to a single peripheral location, to two or three adjacent locations or to two non-contiguous locations in the visual field. It could be demonstrated that subjects used both forms of attentional selection, the zoom lens and the multiple spotlight selection mechanism, within the same experimental paradigm. The comparison between these two attentional mechanisms revealed a decrease in behavioural performance and in activity in striate and extrastriate visual areas as the number of attended locations increased. This effect was independent of the attentional mechanism used. However, a processing efficiency advantage for diving attention versus zooming over the same spatial extent was not only observed in behavioural performance, but was also reflected in greater attentional modulation in the visual cortex.

Furthermore, an analysis of functional and effective connectivity was performed to investigate the neuronal basis of attentional integration of information between two attended but spatially separated stimuli (Haynes et al., 2005). The correlation between measured time courses of different brain areas has been referred to as functional connectivity (Friston et al., 1993b), whereas effective connectivity is defined as the influence one neuronal system exerts over another (Friston et al., 1993a). Using fMRI, attentional integration in terms of effective connectivity between spatially separated locations in the visual field could be shown in early visual cortex, also affecting behavioural performance (Haynes et al., 2005). The deployment of attention among two spatially distinct locations in the visual field led to an integrated information processing and enhanced coupling between the distinct neuronal populations that represented the 
attended visual stimuli. Decreased effective connectivity was observed for unattended locations reflecting functional decoupling of the associated neuronal populations. A correlation between performance and effective connectivity indicated that stronger coupling was associated with enhanced behavioural performance. It was concluded that these changes in coupling between distinct areas of the visual cortex could be a better predictor of performance than the activity within a single cortical locus, suggesting a functional role for attentional integration in the generation of behaviour.

Taken together, several lines of evidence support the view that the deployment of spatial attention is highly flexible in that it can be allocated to distinct locations in the visual field. However, there is a limited number of neuroimaging results addressing the issue of dividing attention among spatially separated regions supporting the multiple spotlight theory. 


\section{Split of attentional resources in human visual cortex}

This study has been published in Visual Neuroscience: Morawetz C, Holz P, Baudewig J, Treue S, Dechent P (2007). Split of attentional resources in human visual cortex. Visual Neuroscience 24: 817-826.

\subsection{Introduction}

Natural visual scenes contain a variety of different and complex objects. As a result of the limited processing capacity of the visual system, they cannot all be processed fully. Therefore, attentional mechanisms perform a selection in favour of behaviourally relevant stimuli for enhanced processing. Attention can be directed to specific parts of the visual field, where information processing is facilitated in contrast to unattended locations (Posner et al., 1980; Driver \& Baylis, 1989; Slotnick et al., 2003). As described in the previous chapter, the study of spatial visual attention has been dominated by "spotlight theories". Using the idea of a spotlight as a metaphor the distribution of attentional resources in visual space has been described by the "single spotlight", "zoom lens", and "multiple spotlights" model (see chapter 3 dor a detailed description).

Numerous functional imaging studies observed attentional modulation in human striate and extrastriate visual cortex (Brefczynski \& DeYoe, 1999; Tootell et al., 1998; Martinez et al., 1999; Somers et al., 1999; Gandhi et al., 1999), even in the absence of any visual stimulus (Kastner et al., 1999). As most of these areas are retinotopically organized, several studies looked for and found a neuronal correlate of the single spotlight (Brefczynski \& DeYoe, 1999; Heinze et al., 1994) and the zoom lens of visual attention (Mueller et al., 2003b). The question of whether the attentional spotlight can be split has been addressed in only a few functional imaging studies so far. Using an electrophysiological measure of attentional allocation (SSVEP) Mueller et al. (2003a) showed that the spotlight of attention could be divided between spatially separated locations for several seconds. So far, only one group has documented attentional modulation in striate and extrastriate visual areas for two 
separately attended locations with a lack of enhanced activity at the irrelevant intermediate regions using fMRI (McMains \& Somers, 2004; 2005).

It has been argued that the ability to divide the attentional spotlight in the presence of two distinct behaviourally relevant locations benefits resource conservation compared to the zoom lens model, where a single spotlight would include task-irrelevant regions between the two relevant locations (McMains \& Somers, 2005). Because of this processing inefficiency, the distance between two attended regions might influence the selection of the attentional mechanism.

Here, I readdress the issue of dividing attention among spatially separated regions close to the centre of the visual field (experiment 1). I also investigate the split of attention across more peripheral target regions (experiment 2) and test the reproducibility of the observed activations (experiment 3). In an experimental design adapted from McMains and Somers (2004) subjects had to deploy their attention either to a single peripheral location or two non-contiguous regions performing a match-mismatch judgement. A split of attention across two distant locations would be associated with increased activity in striate and extrastriate representations of the attended regions, whereas in the intermediate regions, namely the fovea, the attentional modulation would be lower or even absent (Figure 20a). In contrast, a peak activity in the fovea would favor the single spotlight metaphor (Figure 20b). 

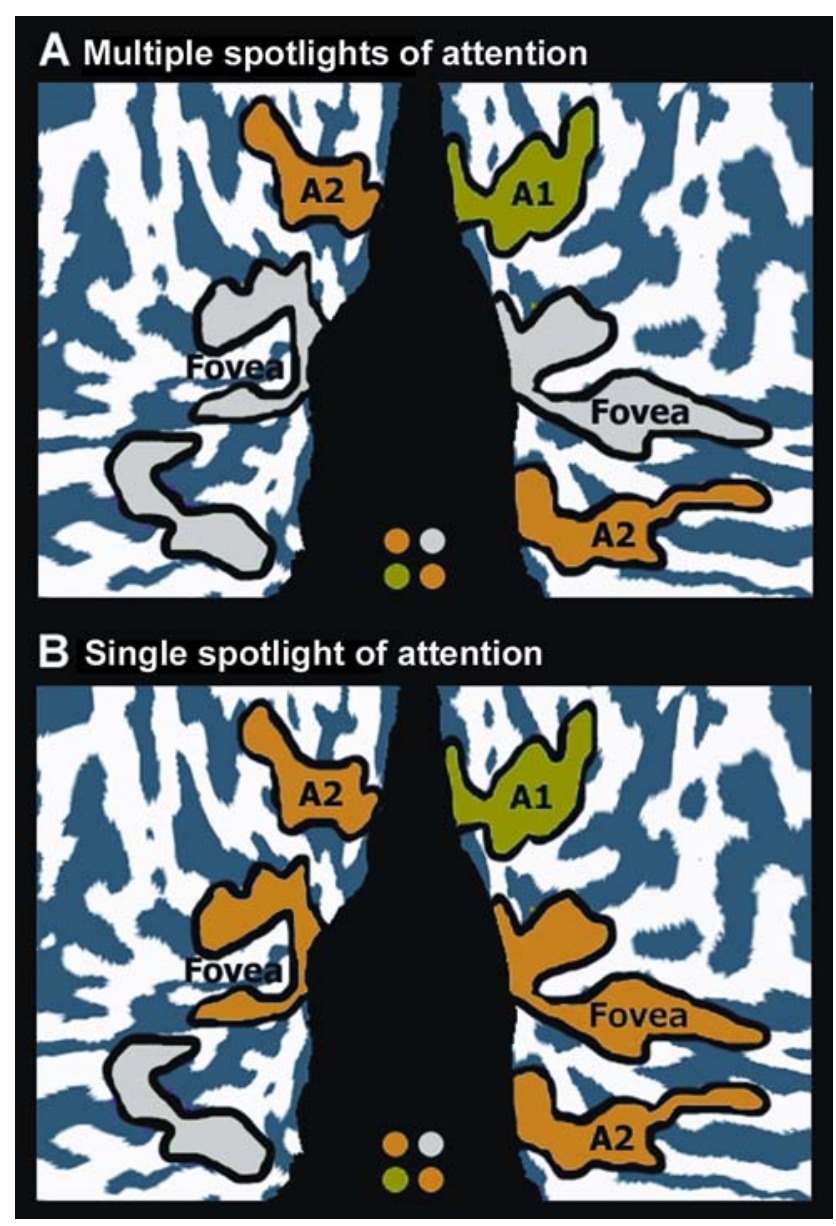

Figure 20

Idealized expected activation patterns displayed on a flatmap-representation of the occipital cortex after cutting along the calcarine sulcus. Coloured dot-schemes show the locations of attended streams in the visual field for Attend1 (green; A1) and Attend2 (orange; A2). Functionally defined ROIs) are indicated in black outlines reflecting the cortical location of the corresponding streams. (A) Multiple spotlights of attention. Enhanced activity is expected in the defined ROIs according to the attended streams presented in the visual field periphery (orange and green). Intermediate regions (fovea) are spared from activation (gray) during the Attend2 task. (B) Single spotlight of attention. Enhanced activity is expected in the defined ROIs according to the attended streams presented in the visual field periphery (orange and green). Intermediate regions (fovea) are activated (orange) during the Attend2 task.

\subsection{Methods}

\subsubsection{Subjects}

The subjects were right-handed, healthy adults, aged 23-41 years (mean $26.9 \pm 5$ years) with normal or corrected to normal vision. All subjects gave written informed consent to participate in the study, which was approved by the local ethics committee. 
In experiment 1 six (same as in experiment 2; 3 females), in experiment 2 twelve (6 females) and in experiment 3 five subjects (from experiment 1 pool; 3 females) participated. None of the subjects was excluded from the study as they all performed above chance level based on the calculated adjusted percent correct answers.

\subsubsection{Attention Tasks}

Visual stimuli consisted of letters and digits displayed in RSVP on LCD-goggles (Resonance Technology, Northridge, USA) using the stimulation software Presentation (Version 9.00, Neurobehavioral Systems, USA). Five RSVP streams were presented simultaneously, one in the centre of the visual field and one in each visual field quadrant. Each stimulus was displayed for $164 \mathrm{~ms}$.

In experiment 1 and 3 the RSVP streams were placed $3.6^{\circ}$ diagonally from central fixation. The letter height subtended a visual angle of $0.6^{\circ}$ for the central stimuli and $1.1^{\circ}$ for the peripheral stimuli. In experiment 2 stimuli were presented at a distance of $8.5^{\circ}$ diagonally from central fixation. The letter height of the stimuli was $0.8^{\circ}$ and $2.3^{\circ}$ in the central and peripheral streams, respectively (Table 1).

Table 1. Details of the three different attention experiments

\begin{tabular}{lccc}
\hline \hline & Experiment 1 & Experiment 2 & Experiment 3 \\
\hline Number of subjects & 6 & 12 & 5 \\
Stimulus duration & $164 \mathrm{~ms}$ & $164 \mathrm{~ms}$ & $164 \mathrm{~ms}$ \\
Peripheral stimulus location* & $3.6^{\circ}$ & $8.5^{\circ}$ & $3.6^{\circ}$ \\
Letter height: Central/peripheral & $0.6^{\circ} / 1.1^{\circ}$ & $0.8^{\circ} / 2.3^{\circ}$ & $0.6^{\circ} / 1.1^{\circ}$ \\
Target location: Attend1 & Lower left & Lower left & All positions \\
Target location: Attend2 & Upper left+ & Upper left + & Upper left + \\
& lower right & lower right & $\begin{array}{l}\text { lower right } \\
\text { Lower left }+\end{array}$ \\
& & & upper right \\
\hline \hline
\end{tabular}

*Distance from visual field center. 
As in the study of McMains and Somers (2004) all experiments consisted of two attention tasks (Attend1 and Attend2, Figure 21a \& 21b). In the Attend1 task subjects had to maintain fixation on the central RSVP stream, where only digits were presented, and monitor one peripheral stream of digits and letters for a predefined target which appeared every 1800-2000ms. The unattended RSVP streams served as distractors. Different digits were defined as targets, which were constant within a run but varied between runs. During experiments 1 and 2 subjects were instructed to monitor only the lower left RSVP stream for targets during the Attend1 task, whereas in experiment 3 the target position varied upon the four different peripheral locations in between trials and runs. In the Attend 2 task subjects had to monitor two peripheral streams in opposing visual field quadrants for the simultaneous appearance of digits, perform match-mismatch judgements and indicate via button press on a custom-made response box whether the digits were the same (match) or different (mismatch). In both experiments target match trials occurred with 50\% probability. Task difficulty varied in between the experiments depending on the distance of the RSVP streams from central fixation. The adjusted frequency of correct answers (\% correct - \% incorrect) was calculated for each participant in order to exclude subjects for whom the adjusted frequency of correct answers was not different from zero (chance level). The behavioral data were analysed using a paired t-test.

Two additional tasks were performed: Passive viewing of the five RSVP streams without a match-mismatch judgement and maintaining Fixation on a central fixation cross displayed on a gray background (Figure 21c). All experiments were implemented in a block design where each of the four conditions (Attend1, Attend2, Passive, Fixation) lasted 40s (including $6 \mathrm{~s}$ of visually displayed written instructions) resulting in a stimulation block of 160 s, which was repeated three times resulting in $8 \mathrm{~min} 20$ s per experimental run. Runs were repeated four to six times. Tasks and blocks were presented pseudo-randomized. Before scanning subjects were trained on the attentional tasks in separate sessions. 

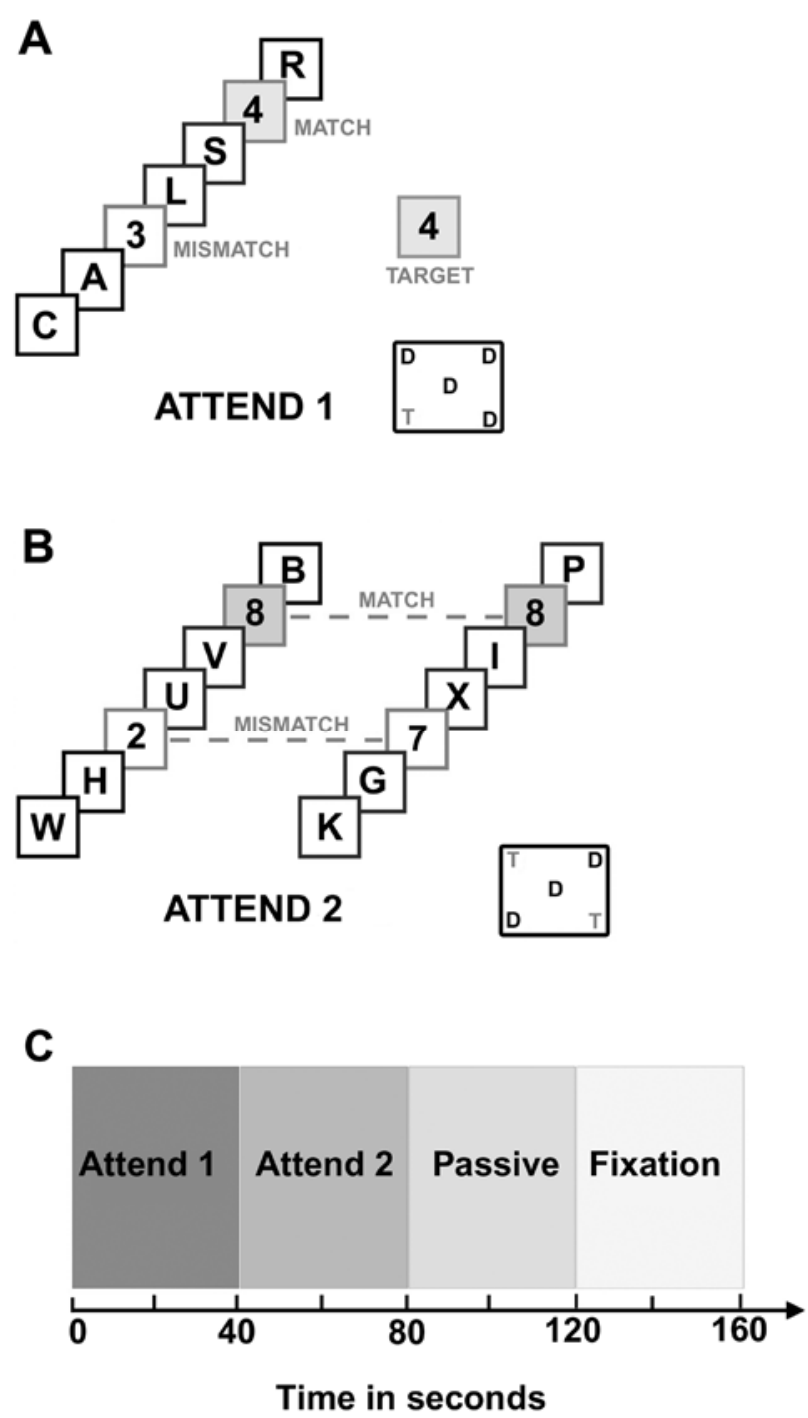

\section{Figure 21}

Attention tasks and experimental design. Five rapid serial visual presentation (RSVP) streams were presented simultaneously. Subjects had to maintain fixation on the central RSVP stream, where only digits were displayed (distractor stream; D). (A) Attend1 task. Subjects had to monitor the lower left stream (target location; $\mathrm{T}$ ) for the appearance of digits and indicate via button press if the digits matched or mismatched the target. Distractor streams had to be ignored. (B) Attend2 task. The upper left and lower right RSVP stream were defined as target locations (T). Simultaneously appearing digits were considered as targets. Participants indicated via button press whether the digits were the same (match) or different (mismatch). (C) The stimulation paradigm employed for functional imaging. One block consisted of four different conditions, each lasting 40s, repeated for three times. Runs were repeated four to six times. 


\subsubsection{Retinotopic Mapping}

Several retinotopically organized visual areas (V1, V2, V3/VP, V3A/V4) in the normal human brain abut one another at the vertical and horizontal meridian representations. Bounderies between these areas can be identified from positions of horizontal and vertical meridians measured using various checkerboard stimuli. In a first step we tested three different stimulation paradigms (wedges, halfchecker board, meridian) in order to produce the most informative retinotopic maps possible according to the aims of the present study in a small group of subjects $(n=4)$. Retinotopic mapping was performed using black-whitereversing checkerboard stimuli flickering at $5 \mathrm{~Hz}$. Each stimulus was presented for $10 \mathrm{~s}$, a central fixation cross was displayed during all trials and one run lasted $6 \mathrm{~min}$.

In a first experiment a clockwise rotating wedge (Figure 22a), a clockwise rotating halfcheckerboard (Figure 41b) and a bow-tie shaped horizontal or vertical visual stimulus (Figure 22b) was used to map angular position in the visual field. Brain regions processing a particular position in the visual field were activated when the reversing checkerboard passed trough that position. After analyis, we decided to use the wedges (bow-tie shaped stimuli) exclusively stimulating the horizontal and vertical meridians of the visual field to determine the borders of the visual areas.

In the present study, three retinotopic mapping experiments were conducted using an annular ring stimulus (eccentricity map), the meridian stimulation (meridian map) and peripheral circular stimuli (target ROIs). The checkerboard probes of the ring stimulus were scaled by the human cortical magnification factor to activate an approximately equivalent area of cortex at all stimulated eccentricities (Van Essen et al., 1984) (Figure 23a). To functionally identify cortical visual areas V1, V2, V3/VP, V3A/V4 the horizontal and vertical meridians of the visual field were selectively stimulated (Figure 23b). The specific peripheral ROIs for the attention tasks (location of RSVP streams) were identified using corresponding circular stimuli (Figure 23c). 

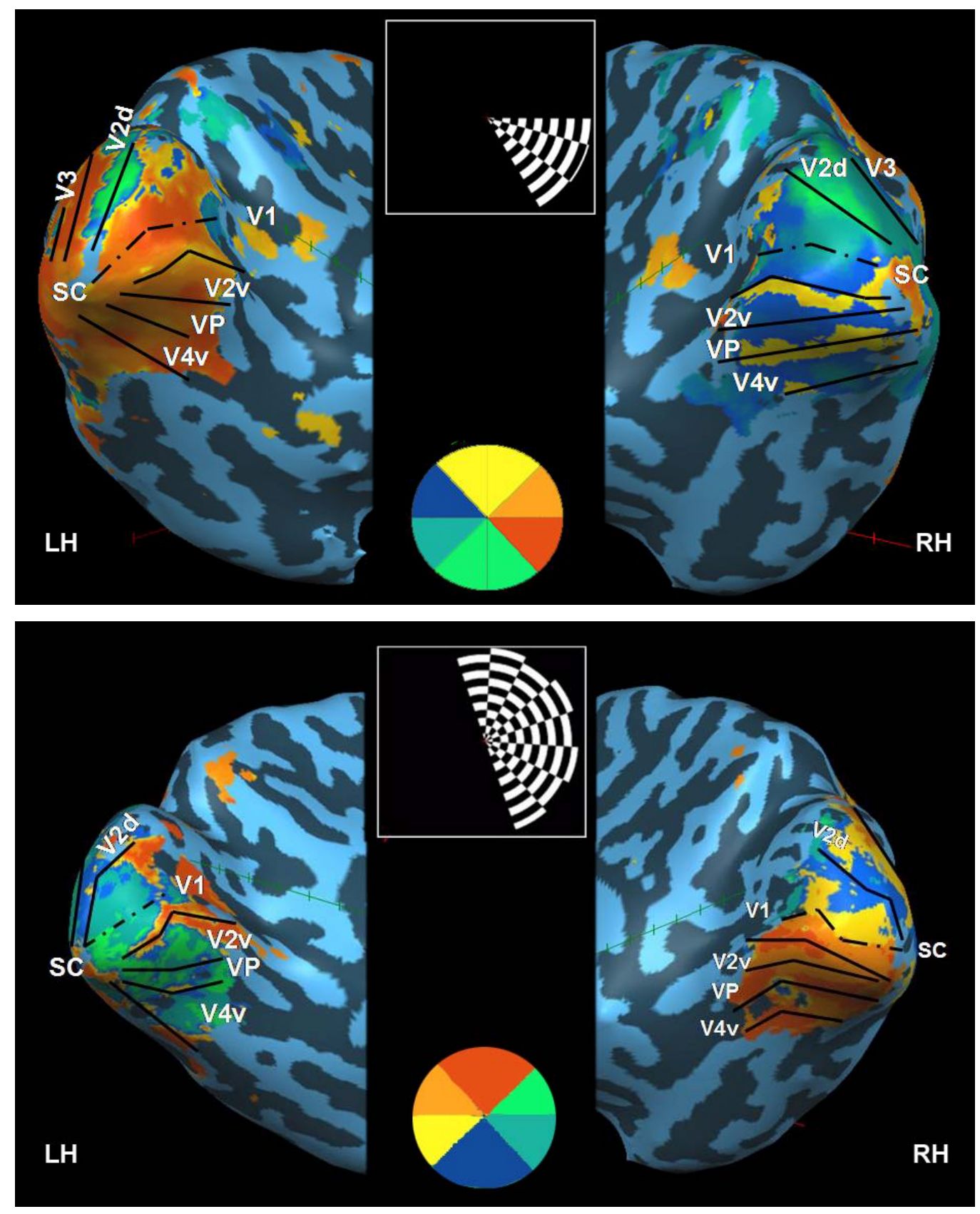

\section{Figure 22}

Inflated reconstruction of segmented cortex of the left and right hemisphere of one subject, showing meridional activation by (a) rotating wedges (inset) and (b) a rotating halfcheckerboard (inset) defining the retinotopic areas (black outlines) on the occipital lobe. Surfaces are viewed from a medio-posterior viewpoint. 


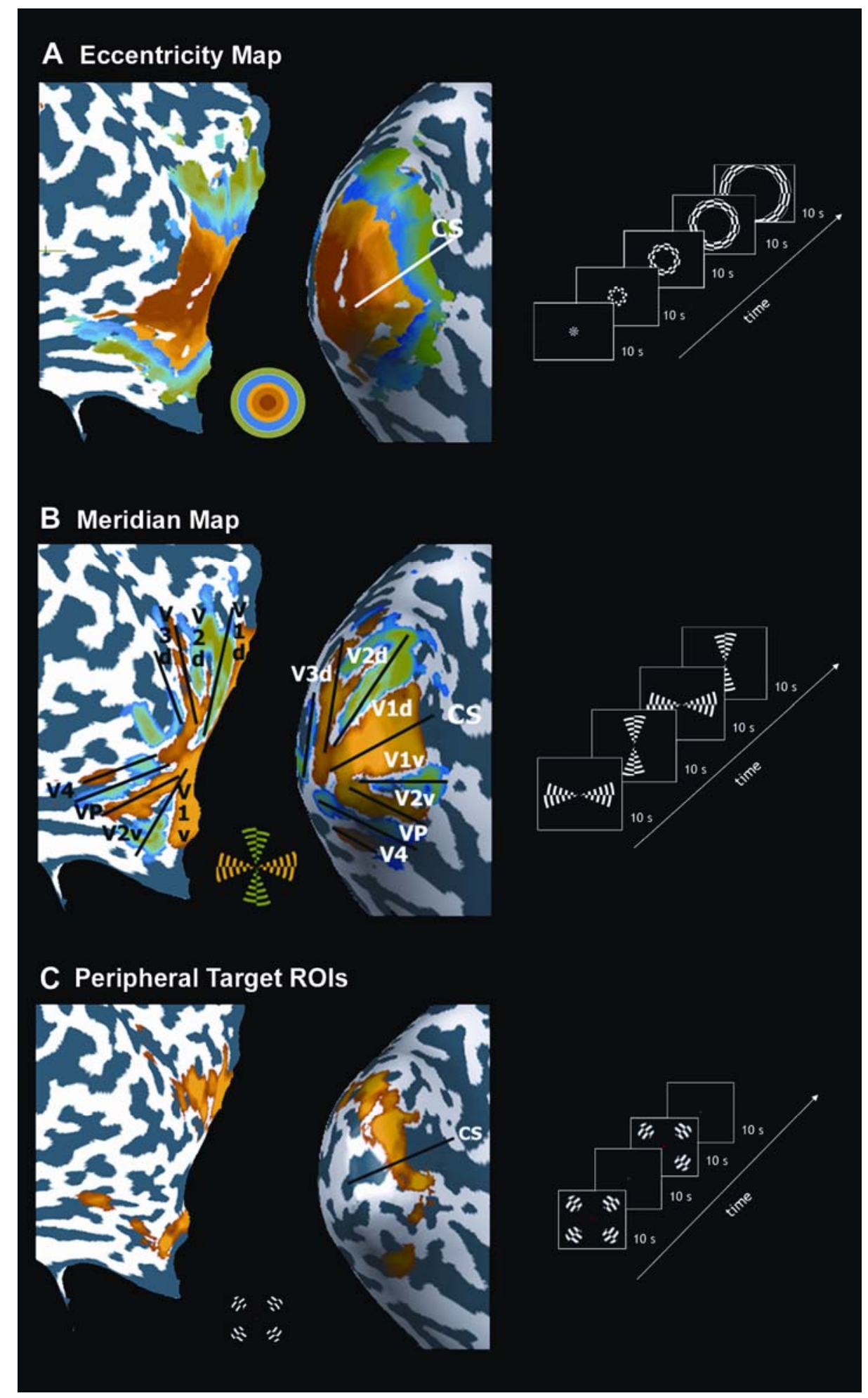

\section{Figure 23}

Retinoptopic mapping (Subject 5) in the left hemisphere displayed on the inflated cortex (right) and a flatmap-representation (left) after cutting along the calcarine sulcus (CS). (A) Eccentricity map. An annular ring stimulus with increasing eccentricity (red to green) results in a traveling wave of activation from the occipital pole to more anterior aspects of the occipital lobe. (B) Meridian map. Selective stimulation of the horizontal (orange) and vertical (green) meridians of the visual field allows for identification of cortical visual areas and respective borders. (C) Peripheral target regions of interest. Selective stimulation of the peripheral ROIs of the visual field with checkerboards allows for the indentification of their representation in the visual cortex (yellowred). 


\subsubsection{Magnetic Resonance Imaging and Data Analysis}

During scanning 250 functional volumes for each run of the attention tasks and 180 functional volumes for the retinotopic mapping experiments were recorded. In a first scanning session the high-resolution 3D T1-weighted as well as the retinotopic mapping datasets were obtained. In a second scanning session subjects performed the different attention tasks.

Using the anatomical data, both hemispheres were individually segmented at the graywhite-matter boundary. After surface reconstruction of this boundary, each hemisphere was inflated, cut along the calcarine sulcus and flattened. The functional scans of the attention tasks were spatially smoothed (Gaussian smoothing kernel, $8 \mathrm{~mm}$ full width half maximum). Retinotopic mapping experiments were analyzed according to standard procedures (Sereno et al., 1995; DeYoe et al., 1996; Tootell et al., 1996). On the basis of correlation analysis of the ROI-mapping data, ROI masks were calculated, projected onto the flat maps and used for the analysis of the attention experiments. The ROI analysis in experiment 1 and 2 was based on the general linear model approach contrasting the attention tasks (Attend1, Attend2) versus the Passive viewing condition. The contrast between the Attend 2 versus Attend 1 task of experiment 1 and 3 and a correlation analysis of the t-values of each ROI for each subject of both experiments was used to test data reproducibility. The obtained activation maps were based on a false discovery rate of $\mathrm{q}(\mathrm{FDR})<0.001$.

\subsubsection{Eye Tracking}

Eye position was monitored during scanning in experiment $1(\mathrm{n}=5)$ and $2(\mathrm{n}=7)$ using a custom-built MR-compatible video camera that provided input to viewpoint eyetracker software (Arrington Research Inc., Scottsdale, Arizona, USA) to ensure that participants maintained fixation on the central stream. After the detection and elimination of blinks in the raw data, an eye position time-course was computed for each condition of each experimental run (Figure 24). 
Fixation

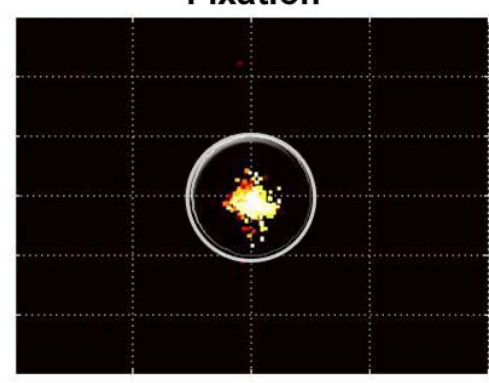

Attend1

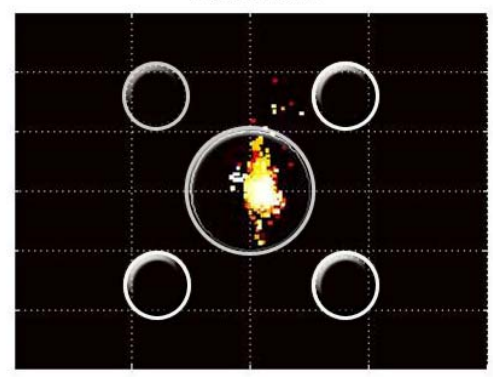

0

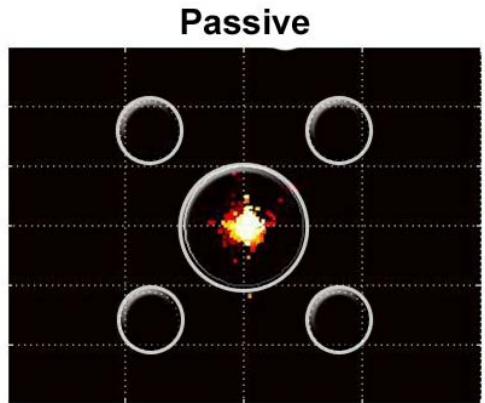

Attend2

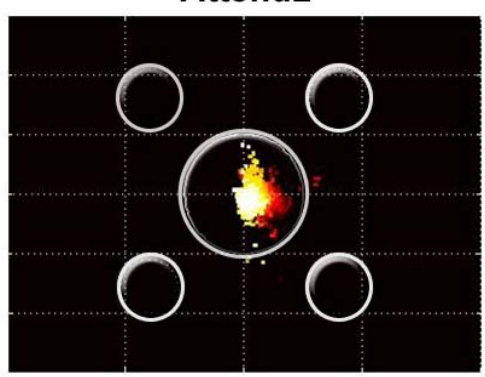

34

Time in sec

\section{Figure 24}

Eye position time-course of one subject for each condition during one experimental run of experiment 2. Coloured dots represent the gaze position in the visual field over time from red (beginning of trial) to yellow (end of trial). White circles indicate the position of the RSVP streams in the visual field.

\subsection{Results}

\subsubsection{Behavioral Results}

Task performance is shown in Figure 25. In experiment 1 subjects performed significantly better on the Attend1 trials $(98 \%)$ than on the Attend 2 trials $(90 \% ; p<0.05)$. In experiment 2 subjects correctly detected an average of $89 \%$ and $86 \%$ of the targets during the Attend 1 and the Attend 2 task, respectively. Mean task performance in experiment 1 was better than in experiment 2 because the RSVP streams were less eccentric. Comparing the same group of subjects in these experiments showed that participants performed significantly lower on both attentional tasks in experiment 2 (Attend1: 86\%; Attend2: 82\%; $<<0.05$ ), when the RSVP streams were presented at more peripheral locations. In experiment 3 the mean task performance for the Attend 1 task was $89 \%$ and for the Attend 2 task $84 \%$ correct. 


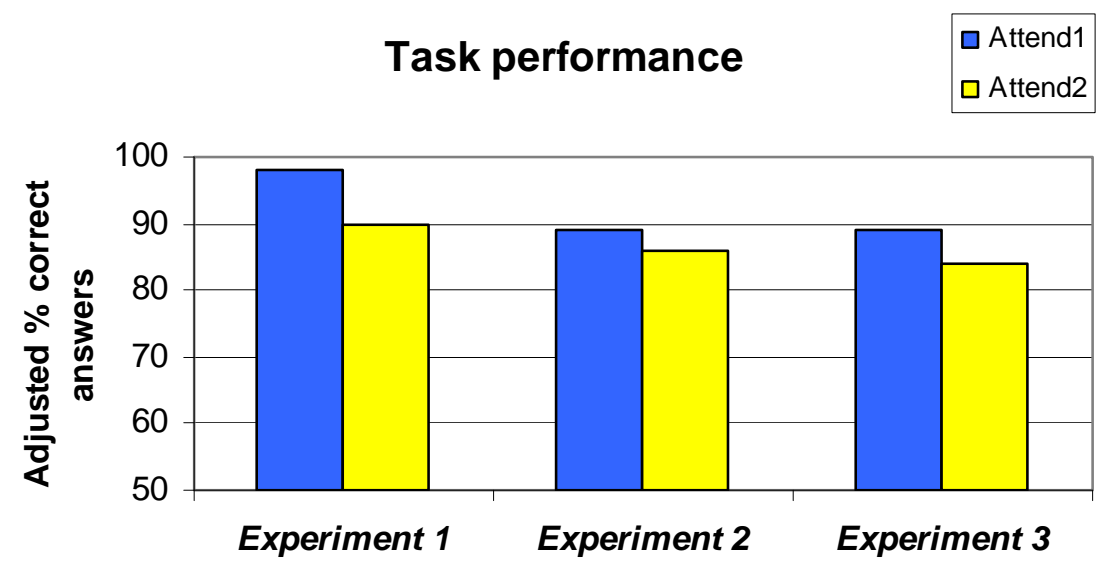

\section{Figure 25}

Adjusted percent correct answers of Attend 1 and Attend 2 for all three experiments.

\subsubsection{Neuroimaging Results}

Contrasting each of the two attentional tasks versus Passive viewing revealed an attentional modulation in striate and extrastriate visual areas (V1, V2, V3/VP, V3A/V4v). Increased activity was observed in the ROIs according to the attended RSVP streams in all experiments. Additionally, intermediate (foveal) and task-irrelevant regions were activated in some subjects. The results of the ROI analyses for each individual are given in Table 3 (experiment 1) and Table 4 (experiment 2).

\subsubsection{Experiment 1}

Increased activation for the Attend1 task (versus Passive viewing) was found in striate and extrastriate visual areas at the retinotopic representation corresponding to the attended location in the lower left quadrant of the visual field. The Attend2 task in contrast to Passive viewing was associated with enhanced activation in the task-relevant regions in the upper left and lower right, with a variable amount of additional activation in the fovea.

The group ROI analysis of experiment 1 (Table 2) revealed increased activity for the Attend 2 task in contrast to Passive viewing in the upper left and lower right ROI compared to the foveal region. Greater activation for the Attend1 task was observed in the upper right ROI compared to the foveal region and the other ROIs. On a single subject basis in all participants the ROI analysis was consistent with the predictions of the multiple spotlights hypothesis. 
Table 2. Region of Interest Analysis of Experiment 1

\begin{tabular}{|c|c|c|c|c|c|c|}
\hline \multirow[b]{2}{*}{ Subject } & \multirow[b]{2}{*}{ Condition } & \multicolumn{5}{|c|}{ Region of Interest (t-value) } \\
\hline & & Upper left & Lower left & Fovea & Upper right & Lower right \\
\hline \multirow[t]{2}{*}{1} & A2 $>$ Passive & 5.3 & 0.2 & 0.1 & -4.4 & 0.4 \\
\hline & A1 $>$ Passive & 4.5 & 3.2 & 2.9 & 4.1 & 2.8 \\
\hline \multirow[t]{2}{*}{2} & A2 $>$ Passive & 8.0 & 6.5 & 6.8 & 5.5 & 7.5 \\
\hline & A1 $>$ Passive & 3.8 & 3.4 & 5.0 & 6.6 & 4.7 \\
\hline \multirow[t]{2}{*}{3} & A2 $>$ Passive & 4.8 & -0.5 & -2.2 & -3.0 & 1.4 \\
\hline & A1 $>$ Passive & 3.6 & -0.2 & -0.5 & 0.5 & 2.8 \\
\hline \multirow[t]{2}{*}{4} & A2 $>$ Passive & 2.8 & 5.7 & 1.5 & 2.3 & 5.5 \\
\hline & A1 $>$ Passive & -0.2 & 3.8 & 2.1 & 5.3 & 2.9 \\
\hline \multirow[t]{2}{*}{5} & A2 $>$ Passive & 3.8 & 3.4 & 4.1 & 0.8 & 4.4 \\
\hline & A1 $>$ Passive & 2.4 & 4.3 & 9.4 & 11.8 & 6.8 \\
\hline \multirow[t]{2}{*}{6} & A2 $>$ Passive & -3.0 & -2.0 & -3.7 & -4.3 & 1.1 \\
\hline & A1 $>$ Passive & -9.6 & -6.4 & -8.1 & -3.4 & -3.5 \\
\hline \multirow[t]{2}{*}{ Group } & A2 $>$ Passive & $3.6 \pm 3.7 *$ & $2.2 \pm 3.5$ & $1.1 \pm 3.9$ & $-0.5 \pm 4.0$ & $3.4 \pm 2.9^{*}$ \\
\hline & A1>Passive & $0.8 \pm 5.3$ & $1.3 \pm 4.1$ & $1.8 \pm 5.9$ & $4.2 \pm 5.2^{*}$ & $2.7 \pm 3.5$ \\
\hline \multicolumn{7}{|c|}{$\begin{array}{l}\text { Asterisk represent post hoc analyses comparing the upper left and lower right position versus the } \\
\text { foveal region in the Attend2 (A2) }>\text { Passive contrast and the upper right position versus the lower left } \\
\text { and foveal region in the Attend1 (A1) }>\text { Passive contrast using a one-tailed paired t-test }(* \text { p }<0.05) \text {. } \\
\text { Group results are presented as Mean } \pm \text { Standard Deviation. The gray shading indicates task-relevant } \\
\text { ROIs. The dashed frames indicate the ROIs critical regarding the assignment to the single spotlight or } \\
\text { multiple spotlights model. }\end{array}$} \\
\hline
\end{tabular}

\subsubsection{Experiment 2}

Similar to experiment 1, the Attend1 task resulted in increased activity according to the attended RSVP stream. Contrasting the Attend2 task versus the Passive viewing condition yielded significant increases in activation in the ROIs corresponding to the peripheral RSVP streams with a variable amount of additional activation in the fovea. Representative single subject activation maps are shown in Figure 26.

The group ROI analysis of experiment 2 (Table 4) revealed increased activity in the two target locations of the Attend2 task in comparison to the foveal region. For Attend1 enhanced activity compared to the fovea and the other regions was obtained in the upper right ROI. On a single subject basis in ten of twelve participants (subjects 1-10) the ROI 
analysis was consistent with the predictions of the multiple spotlights hypothesis. One participant (subject 6) showed an overall decreased activity in all ROIs, but since the decrease was largest in the ROI in the centre of the visual field, the effect was in line with the multiple spotlights hypothesis. In two participants (subjects 11 and 12) activity in the foveal region was higher than in the peripheral regions relevant for the Attend 2 task, in agreement with a single spotlight of attention.

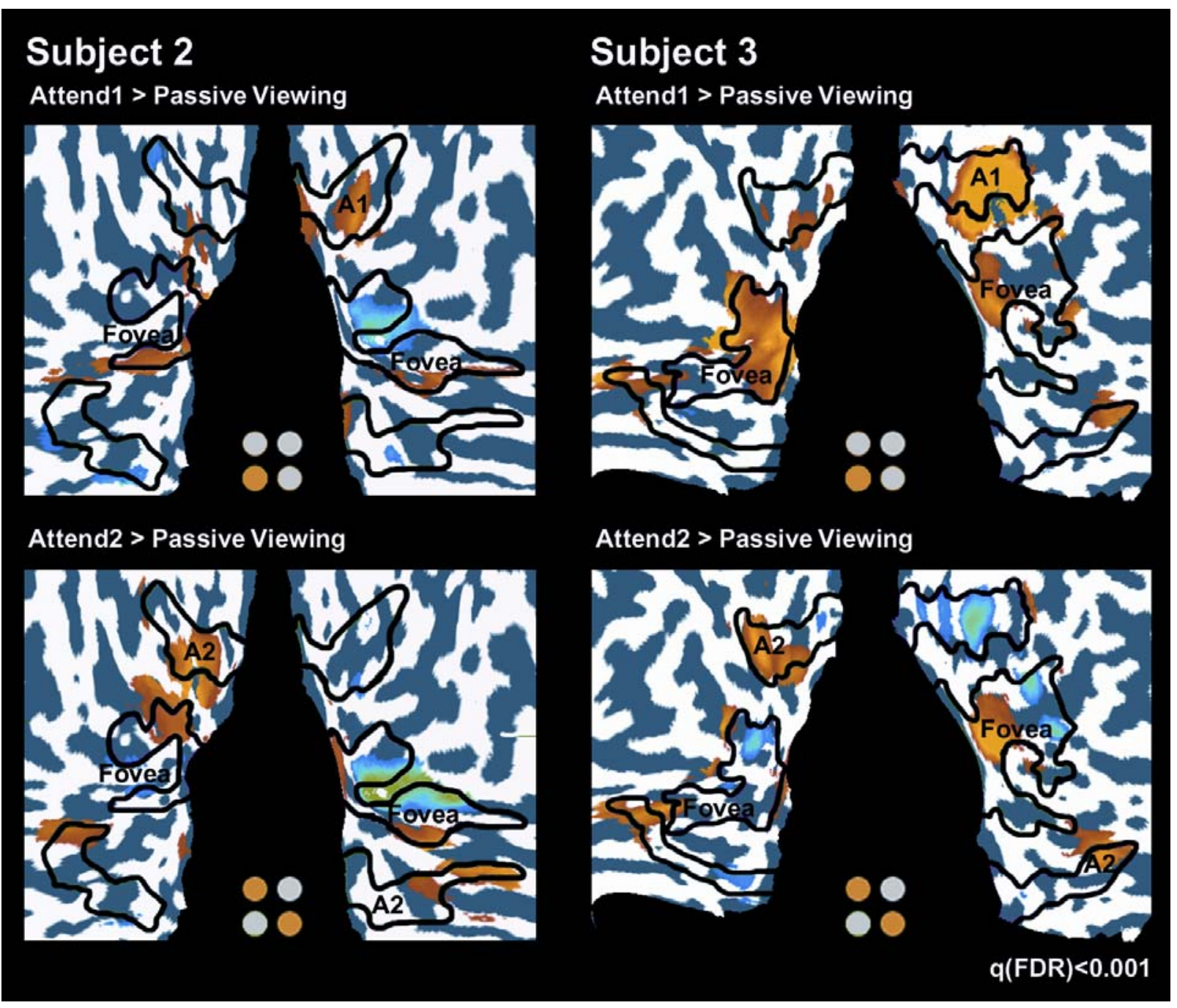

\section{Figure 26}

Results of experiment 2. Individual activation maps of two subjects contrasting Attend1 and Attend2 (yellow-red), respectively, versus Passive viewing (green-blue). Coloured dot-schemes show the locations of attended RSVP streams in the visual field quadrants for Attend1 and Attend2 (orange). Functionally defined ROIs are indicated in black outlines reflecting the representation of corresponding RSVP streams. For Attend1 (A1) enhanced activity was obtained above the calcarine sulcus in the right hemisphere corresponding to the attended location. Increased activation for the Attend2 (A2) task was observed in the corresponding ROIs according to the attended RSVP streams with a variable amount of additional activation in the fovea. 
Table 3. Region of Interest Analysis of Experiment 2

Region of Interest (t-value)

\begin{tabular}{|c|c|c|c|c|c|c|}
\hline Subject & Condition & Upper left & Lower left & Fovea & Upper right & Lower right \\
\hline \multirow[t]{2}{*}{1} & A2 $>$ Passive & 6.2 & 2.0 & -2.7 & -1.0 & 0.6 \\
\hline & A1 $>$ Passive & 2.3 & 2.3 & -3.0 & 3.9 & 1.0 \\
\hline \multirow[t]{2}{*}{2} & A2 $>$ Passive & 3.3 & 0.5 & 2.4 & -0.7 & 1.6 \\
\hline & A1 $>$ Passive & -0.3 & -2.1 & $\overline{3.0}$ & 3.1 & -0.9 \\
\hline \multirow[t]{2}{*}{3} & A2 $>$ Passive & 3.6 & 0.2 & 0.8 & -3.9 & 1.3 \\
\hline & A1 $>$ Passive & 2.2 & 0.8 & 3.7 & 4.2 & 2.3 \\
\hline \multirow[t]{2}{*}{4} & A2 $>$ Passive & 9.9 & 9.5 & 4.3 & 3.6 & 9.0 \\
\hline & Al $>$ Passive & 7.0 & 8.4 & 4.3 & 11.3 & 10.9 \\
\hline \multirow[t]{2}{*}{5} & A2 $>$ Passive & 1.3 & 4.5 & -6.0 & 3.3 & 4.3 \\
\hline & A1 $>$ Passive & -1.2 & 2.3 & 5.5 & 10.3 & 6.8 \\
\hline \multirow[t]{2}{*}{6} & A2 $>$ Passive & -3.7 & -2.0 & -5.6 & -9.0 & -2.9 \\
\hline & Al $>$ Passive & -8.4 & -5.3 & -4.4 & -1.6 & -4.2 \\
\hline \multirow[t]{2}{*}{7} & A2 $>$ Passive & 1.8 & -1.2 & -1.0 & -1.4 & 0.3 \\
\hline & A1 $>$ Passive & -2.0 & -1.3 & 0.2 & 0.9 & -0.2 \\
\hline \multirow[t]{2}{*}{8} & A2 $>$ Passive & 5.5 & 5.0 & 4.4 & 1.2 & 5.3 \\
\hline & Al $>$ Passive & 2.0 & 2.1 & $\overline{4.4}$ & 6.7 & 5.7 \\
\hline \multirow[t]{2}{*}{9} & A2 $>$ Passive & 2.2 & 0.7 & 0.8 & -1.1 & 2.0 \\
\hline & A1 $>$ Passive & -0.8 & -0.3 & 0.6 & 1.0 & 0.5 \\
\hline \multirow[t]{2}{*}{10} & A2 $>$ Passive & 0.6 & 3.7 & -0.7 & -2.5 & 0.7 \\
\hline & A1 $>$ Passive & -1.3 & 4.6 & -2.1 & 1.0 & 3.3 \\
\hline \multirow[t]{2}{*}{11} & A2 $>$ Passive & 1.4 & 1.4 & 1.5 & -0.5 & 1.4 \\
\hline & A1 $>$ Passive & 1.1 & 1.8 & 2.3 & 2.2 & 2.9 \\
\hline \multirow[t]{2}{*}{12} & A2 $>$ Passive & 5.6 & 2.8 & 6.6 & 0.5 & 1.2 \\
\hline & A1 $>$ Passive & 5.0 & 6.4 & $\overline{7.4}$ & 5.1 & 0.7 \\
\hline \multirow[t]{2}{*}{ Group } & A2 $>$ Passive & $3.1 \pm 3.4^{* *}$ & $2.3 \pm 3.1$ & $0.4 \pm 3.9$ & $-1.0 \pm 3.3$ & $2.1 \pm 3.0^{\dagger}$ \\
\hline & Al $>$ Passive & $0.5 \pm 3.9$ & $1.6 \pm 3.7$ & $1.8 \pm 3.6$ & $4.0 \pm 3.9^{* *}$ & $2.4 \pm 4.0$ \\
\hline
\end{tabular}

Asterisk represent post hoc analyses comparing the upper left and lower right position versus the foveal region in the Attend2 (A2)>Passive contrast and the upper right position versus all other locations in the Attend1 (A1) $>$ Passive contrast using a one-tailed paired t-test $(\mathrm{p}=0.07 ; * * \mathrm{p}<0.01)$. Group results are presented as Mean \pm Standard Deviation. The gray shading indicates task-relevant ROIs. The dashed frames indicate the ROIs critical regarding the assignment to the single spotlight or multiple spotlights model. 


\subsubsection{Data Reproducibility}

To determine if the pattern of activation following attentional allocation to specific spatial locations is robust, fMRI results of the same subjects were compared across different scanning sessions (experiments 1 and 3). Activation maps of experiment 1 closely matched the pattern produced in experiment 3 during the same task condition contrasting the Attend 2 versus the Attend 1 task. These results were comfirmed by a correlational analysis of the t-values of each ROI for each subject of experiment 1 and 3 revealing a significant positive correlation $\left(\mathrm{r}^{2}=0.81\right)$ between the two datasets indicating a high reproducibility across different runs (Figure 27).

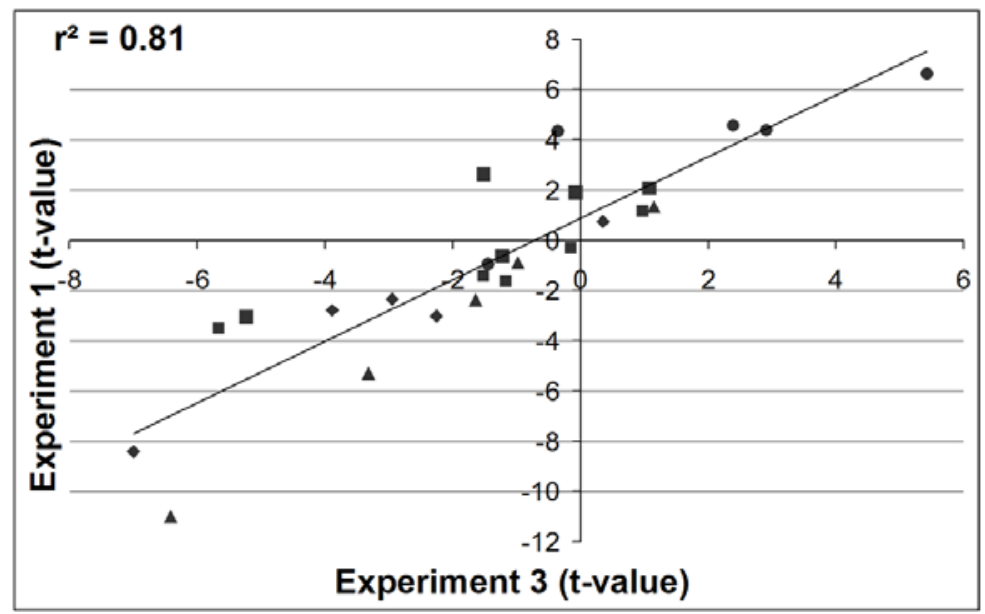

\section{Figure 27}

Data reproducibility. Correlation analysis of t-values of each ROI for each subject of experiment 1 and 3 contrasting Attend2 versus Attend1, indicating a high reproducibility. Different symbols represent the five different subjects.

\section{$\underline{4.4 \text { Discussion }}$}

We investigated whether the simultaneous monitoring of two spatial positions in opposing quadrants of the visual field led to the deployment of two distinct spotlights of attention or whether human subjects covered the two locations with a single appropriately shaped focus of attention. Our results support the presence of multiple spotlights of attention (McMains \& Somers, 2004) in demonstrating that the attentional focus can be divided when two target locations are separated by $7^{\circ}$ of visual angle. Furthermore, our findings reveal that a split of attention can be achieved for a stimulus separation as large as $17^{\circ}$. 
Our findings show that mean task performance for both attentional tasks was higher in experiment 1 than in experiment 2, where the stimuli were presented more peripherally, which is in line with previous studies reporting that multiple targets are easier and faster to identify when they are close together (Hoffman \& Nelson, 1981; Hoffman et al., 1983; Eriksen \& St. James, 1986; Mueller et al., 2003b). However, this result is not attributable to an eccentricity effect, because the letter size of the stimuli was adjusted according to their eccentricity. The observed lower task performance in experiment 3 compared to experiment 1 most likely reflects the fact that the target positions varied in between trials and runs, thereby inducing an increase of task difficulty.

In agreement with previous studies (Tootell et al., 1998; Watanabe et al., 1998; Brefczynski \& DeYoe, 1999; Gandhi et al., 1999; Somers et al., 1999; Kastner et al., 1998; Chawla et al., 1999) we found an attentional modulation in striate (V1) and extrastriate (V2, V3/VP, V4v, V3A) visual areas contrasting the Attend2 task versus Passive viewing. Based on group ROI analyses of experiment 1 and 2 increased activation was observed in the regions corresponding to the attended RSVP streams, namely the cortical representation of the upper left and lower right region of the visual field, in comparison to intermediate regions. Therefore, our results are consistent with the multiple spotlights theory and previously reported findings (McMains \& Somers, 2004) in demonstrating attentional modulation in the task-relevant regions for the Attend2 task versus Passive viewing with lower or even absent attentional effects in intermediate regions. The observed activation in the foveal region during the Attend2 task can be accounted for by two adjacent spotlights of attention. Psychophysical experiments have shown that the ability to shrink the spotlight of attention is limited (Intriligator \& Cavanagh, 2001). Similarly, physiological experiments have shown that the neuronal correlate of spatial attention is a broad distribution of neuronal responsivity that is biased towards the attended location, more than a sharply delimited area of modulation (Womelsdorf et al., 2006). Such a broad profile of two adjacent spotlights of attention would create substantial overlap, resulting in an attentional modulation in the intermediate regions. In our experiments the foveal activation would therefore strongly depend on the size of the two spotlights. Increased activity in the ROIs corresponding to the attended streams with sparing of the fovea represents two separable spotlights (peaks of activity) at a given statistical threshold (Figure 28a), whereas activity in the foveal region can be explained by an overlap of two spotlights (Figure 28b), as long as the observed activity in the foveal region is lower than in the attended task-relevant regions. These patterns of results were observed in all but two 
of our subjects. For these two subjects our ROI analysis of the single subject data showed a peak activity in the foveal region. This finding could be attributed either to a single attentional focus facilitating information processing within a unitary circumscribed region of the visual field (Eriksen \& St. James, 1986; LaBerge, 1983; Duncan, 1984; Heinze et al., 1994; McCormick et al., 1998) or to two wide spotlights (Figure 28c).
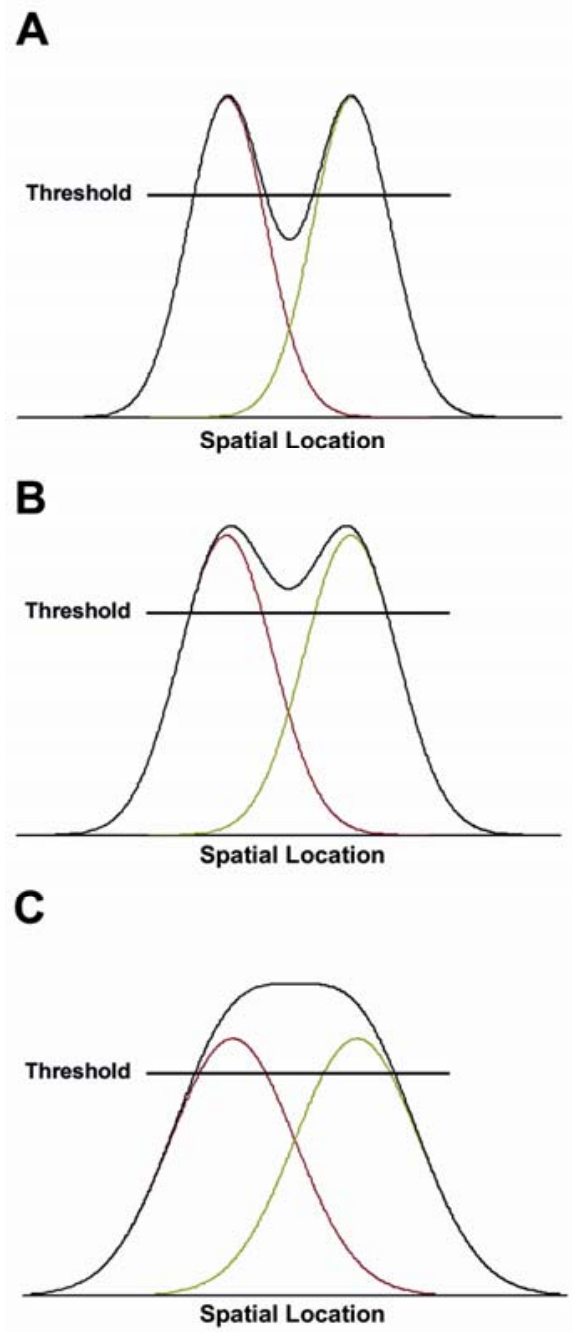

\section{Figure 28}

Separability of attentional spotlights. Neuronal response to multiple attended locations. The black line indicates a putative threshold of the fMRI analysis. (A) Two separable peaks of activity (red and green curve) with sparing of intermediate regions. (B) Two attentional spotlights at the same spatial location as in A, but with larger width, result in a net amount (black curve) of additional activity in intermediate regions. (C) With a further increase of width, overlapping spotlights of attention might result in a peak activity in intermediate regions.

It should be pointed out that it is possible to imagine that the fovea shows weaker attentional modulation than matched peripheral locations. For psychophysics this does not seem to be the case as cueing a foveal location results in the same speed-up of reaction times as the cueing of a peripheral location (Handy \& Khoe, 2005) but the same study 
reports lower EEG activation in the foveal versus the eccentric cueing conditions. While we cannot exclude a possible difference in the susceptibility to attentional modulation (and therefore the BOLD-activation) between fovea and periphery McMains and Somers (2005) documented comparable attentional modulations in the foveal and a peripheral region during a task, where attention had to be directed to these locations simultaneously.

The possibility of a serial model of attention switching across locations is ruled out by our experimental design with stimulus durations of $164 \mathrm{~ms}$ as previous experiments have demonstrated minimum switching times in the range of 200 to $500 \mathrm{~ms}$ (Reeves \& Sperling, 1986; Weichselgartner \& Sperling, 1987; Peterson \& Juola, 2000; Duncan, 1994). Furthermore, the eye tracking data revealed stable fixation to the central RSVP stream with no indication of involuntary saccades toward the attended location.

fMRI exhibits a large variability in the detected hemodynamic responses across fMRI sessions of the same subject and across subjects (Aguirre et al., 1998; McGonigle et al., 2000; Waldvogel et al., 2000). To investigate the within-subject test-retest reproducibility of the obtained activation maps, five subjects performed the same set of tasks during two fMRI sessions on separate days. The comparison of respective activation maps and a correlation analysis of ROI data yielded comparable results across different scanning sessions indicating a high reproducibilty.

In summary, the present study demonstrates the ability of humans to split the attentional spotlight to cover separate locations, creating retinotopically specific double-peaked attentional modulation profiles in striate and extrastriate visual areas. The observed attentional modulation in the intermediate foveal regions likely results from the overlap of attentional spotlights that are wider than the behaviourally relevant locations necessitate. 


\section{Interaction between emotion and attention}

„Let's not forget that the little emotions are the great captains of our lives and we obey them without realizing it."

Vincent Van Gogh

"The chilling wind of cold cognition is giving way to the warmer breeze of cognitive neuroscience that recognizes the crucial functions of emotion and to an affective neuroscience that appreciates its inextricable intertwining with cognition.”

Richard J. Davidson

Happiness, fear, euphoria, ecstasy, sadness, anger, depression, anxiety - these are just a few emotions that colour our lives and contribute to the richness of our experiences. Emotions imbue our actions with passion and character, they modulate memory, facilitate decision making, influence learning, and provide the motivation for critical action in the face of environmental incentives. Moreover, emotions are a key component for many of the fundamental dimensions of personality.

Emotions are valenced responses to external stimuli and/or internal mental representations that (1) involve changes across multiple response systems (e.g. experiential, behavioural, peripheral physiological), (2) are distinct from moods, in that they often have identifiable objects or triggers, (3) can be either unlearned responses to stimuli with intrinsic affective properties (e.g. an unconditioned response to an aversive shock) or learned responses to stimuli with acquired emotional value (e.g. a conditioned response or stimulus-reward association), (4) can involve multiple types of appraisal processes that assess the significance of stimuli to current goals, that (5) depend upon different neuronal systems (Ochsner \& Gross, 2005).

Emotional signals can be considered as aspects of both an emotional response and social communication. There are multiple schemes for categorizing emotions (Table 3). The 
perspective adopted in this chapter is influenced by a psychoevolutionary approach that reflects a rediscovery of Darwin's views on emotion (Darwin, 1965). Crucial in this regard is the affect programs concept (Ekman, 1982; Ekman \& Friesen, 1971). This concept proposes several distinct emotions, termed primary or basic emotions, that include fear, anger, happiness, sadness, disgust, and surprise. These emotions are expressed in multicomponent behavioural patterns including facial, motor, vocal, endocrine, and automatic responses. Most brain structures that participate in the recognition of basic emotions involve both perceptual processing (identifying the geometric configuration of facial features in order to discriminate among different stimuli on the basis of their appearance) and recognition of the emotional meaning of a stimulus (knowing that a certain expression signals fear). Next to the primary emotion category, emotions such as pride, shame, and guilt, among others, have been proposed to represent a seperate category of higher cognitive or secondary emotions, also called social emotions (Griffiths, 1997; Damasio, 1995). Distinguishing features of this category are greater involvement of higher cognition and the fact that they arise almost exclusively within the context of interpersonal relationships.

Table 4

\section{Classification schemes for emotion.}

\begin{tabular}{|c|c|c|c|c|c|}
\hline $\begin{array}{l}\text { Behavioral } \\
\text { states }\end{array}$ & $\begin{array}{l}\text { Motivational } \\
\text { state }\end{array}$ & $\begin{array}{l}\text { Moods, } \\
\text { background } \\
\text { emotions }\end{array}$ & $\begin{array}{l}\text { Emotion } \\
\text { systems }\end{array}$ & $\begin{array}{l}\text { Basic } \\
\text { emotions }\end{array}$ & Social emotions \\
\hline Approach & Reward & Depression & Seeking & Happiness & Pride \\
\hline \multirow[t]{8}{*}{ Withdrawal } & Punishment & Anxiety & Panic & Fear & Embarrassment \\
\hline & Thirst & Mania & Rage & Anger & Guilt \\
\hline & Hunger & Cheerfulness & Fear & Disgust & Shame \\
\hline & Pain & Contentment & & Sadness & Maternal love \\
\hline & Craving & Worry & & Surprise & Sexual love \\
\hline & & & & (Contempt) & Infatuation \\
\hline & & & & & Admiration \\
\hline & & & & & Jealousy \\
\hline
\end{tabular}

Emotions are situated in a continuum of response classifications. The more primitive classes, towards the left, pertain to emotional reactions, whereas the more complex classes, towards the right, pertain to social communication. Typically, experiments with animals are based on a scheme relying on reward and punishment, whereas research in humans has usually adopted the concept of the basic emotions, and psychiatric or social psychological studies have utilized even more complex constructs such as social emotions (from Adolphs, 2002a). 
As psychology transformed from the science of the mind (James, 1950; Wundt, 1897) into the science of behaviour (Skinner, 1953; Watson, 1919), an important topic slipped from scientific view: the subjective experience of emotion. Since then the neuronal substrates of human emotion have received considerable attention. The rapid development of brain imaging techniques, such as fMRI and PET, in the past decade has given rise to a renaissance in the study of emotion. Although affective neuroscience tries to identify the specific subcomponents that constitute emotions and their underlying neuronal basis, the prevailing wisdom remains that "emotion researchers need to figure out how to escape from the shackles of subjectivity if emotion research is to thrive" (LeDoux, 2000, p.156). Therefore, a growing number of studies have adopted the useful complementary approach of including measures of individual differences in the analysis to uncover brain areas whose activity covaries with these measures, for example, personality measures or subjective ratings of emotional responses. Regional brain activity associated with emotion processing can be influenced by a range of individual differences, including differences in personality, dispositional affect, gender, and genotype (Hamann \& Canli, 2004). Correlations between individual difference measures and regional brain activity help to overcome the subjectivity of emotional experiences in order to proceed in the quest to reveal the neuronal bases of emotion processing, generation and regulation.

The cognitive control of emotion plays a crucial role in human adaptive behaviour depending on interactions between prefrontal and cingulate control systems and cortical and subcortical emotion-generative systems. As outlined earlier, selective attention devotes cognitive resources to behavioural relevant stimuli and events in order to achieve enhanced processing of them. A primary way to determine the importance of a stimulus or event is to evaluate the emotional significance (Compton, 2003). Emotionally significant stimuli receive enhanced processing via two different operating attentional mechanisms. The first one evaluates emotional significance preattentively or 'automatically', whereas the second one gives these significant stimuli priority in the competition for selective attention. However the emotional significance of a stimulus varies within individuals, several stimuli such as snakes, spiders, and human faces, are supposed to be extremely emotionally significant to most individuals. Faces are probably the most biologically and socially significant visual stimuli in the human environment, and might therefore be expected to receive enhanced processing.

The concept that emotion and cognition are separate systems that seldom interact, has a long history in Western philosophy and science. However, the past two decades have seen 
a remarkable shift in this view as behavioural and neuroscience data have demonstrated that it is inherently problematic to emphasize a model which tries to classify a certain brain region to be purely cognitive or affective. Cognition and emotion are both part of a dynamic system, which is based on the interaction and cooperation of a distributed network of contributing brain areas (Pessoa, 2008; Damasio, 1995; Phelps, 2006; Dolan 2003; Drevets \& Raichle, 1998; Vuilleumier \& Driver, 2007), which is illustrated in detail in Figure 29 for the face perception and attention systems.

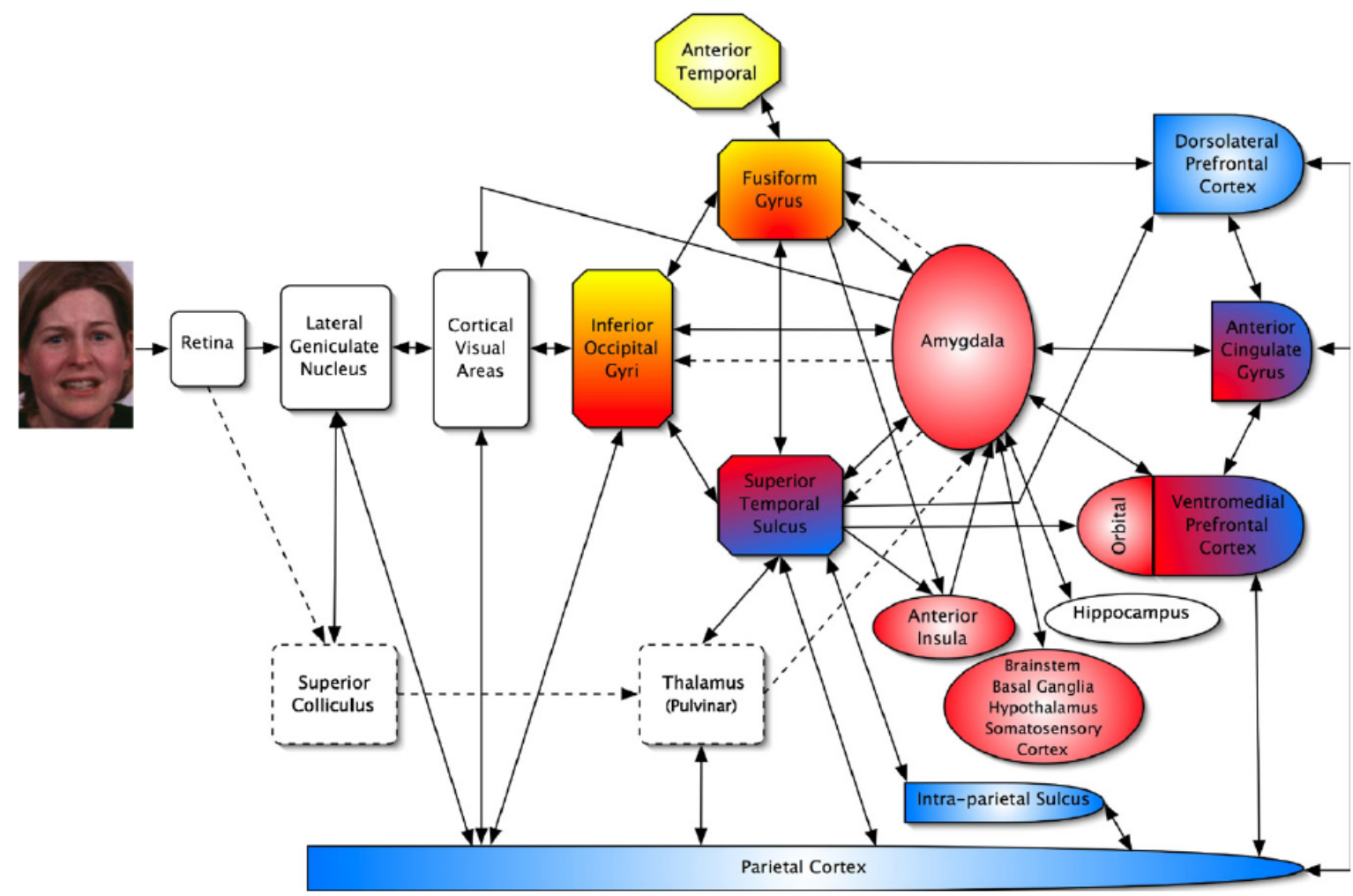

\section{Figure 29}

Face perception and attention systems. The three rectangles with bevelled edges indicate the core system for face perception (Haxby et al., 2000). Areas shaded in yellow represent regions involved in processing identity and associated semantic information, areas in red represent regions involved in emotion analysis (Adolphs, 2002b), and those in blue reflect the fronto-parietal cortical network involved in spatial attention (Hopfinger, et al. 2000). Solid lines indicate cortical pathways and dashed lines represent the subcortical route for rapid and/or coarse emotional expression processing (from Palermo \& Rhodes, 2006).

Spatial attention is controlled by both exogenous bottom-up factors, such as stimulus salience and endogenous top-down factors, such as the intentions and goals of the observer (Corbetta \& Shulman, 2002; Desimone \& Duncan, 1995; Yantis, 1998). Information about facial expressions from visual cortical areas (Figure 29, solid lines) and the pulvinar (Figure 29, dashed lines) is evaluated for emotional significance by the amygdala. The 
amygdala has extensive re-entrant projections to all regions in the visual cortex, so once activated it is able to directly regulate cortical perceptual processing and thus the kind of input it continues to receive (Amaral et al., 1992; Davis \& Whalen, 2001; LeDoux, 2000). Therefore, the amygdala also influences visual cortical processing indirectly, via reciprocal connections to regions of orbital and ventromedial prefrontal cortex (PFC) (Barbas, 2000; Bush et al., 2000; Holland \& Gallagher, 2004; Stefanacci \& Amaral, 2002). Regions in the 'affective' VMPFC are supposed to assess the emotional value of stimuli (Davidson \& Irwin, 1999; Keane et al., 2002) and might be the source of top-down guidance of selective attention to emotional stimuli (Yamasaki et al., 2002). It has been demonstrated that PFC responses are linked with amygdala responses, with enhanced activity in prefrontal regions correlated with an attenuation of amygdala activation that occurred during cognitive evaluation of facial expressions (Hariri et al., 2000). Top-down stimulus selection might also occur via a dorsal route from posterior parietal cortex to dorsolateral PFC (DLPFC) (Holland \& Gallagher, 2004; Yamasaki et al., 2002). The dorsolateral 'cognitive' region has reciprocal connections with cortical sensory and association areas, but not directly with the amygdala, and is part of a distributed attentional network involved in selecting and maintaining task-relevant representations in working memory regardless whether those are emotional or not (Figure 29, blue shading) (Banich et al., 2000; Curtis \& D'Esposito, 2003; Compton, 2003; Yamasaki et al., 2002). The information obtained from 'affective' and 'cognitive' streams is supposed to be integrated by the anterior cingulate gyrus and more lateral PFC regions (Fichtenholtz et al., 2004; Yamasaki et al., 2002; Gray et al., 2002). Finally, it has to be pointed out that various subcortical and cortical regions interact in a complex, but close manner to process visual information. During attentionally demanding cognitive tasks affective regions are deactivated, whereas during emotional information processing responses in cognitive regions are suppressed (Drevets \& Raichle, 1998). This integrative operation between emotion and cognition is necessary for adaptively guiding behaviour.

In the first part of this chapter a brief overview of the most relevant structures involved in the perception of emotion in faces is provided and in the second part evidence concerning the issue of the dependence of face encoding processes on attentional resources is reviewed. 


\subsection{Anatomical and functional correlates of emotion recognition in faces}

Face perception is one of the most highly developed visual skills of human beings. A person's face is not only the physical stimulus that is most closely associated with the representation of that person, it rather allows rapid access to information about that person that is essential for effective social interactions. A face conveys different types of information like gender, age, identity, emotion, ethnicity or other socially relevant categories. There is considerable evidence that recognition of most of these classes of attributes can be dissociated (Bruce \& Young, 1986) and is accomplished by a distributed network of brain regions (Haxby et al., 2000; Ishai et al., 2005). The FFA (Kanwisher et al., 1997) is a key node in distributed neuronal systems for face perception but, by itself the FFA cannot provide a useful account for processes such as the recognition of expression and emotional resonance. In addition to the fusiform gyrus, extrastriate visual regions like superior temporal sulcus (STS) are important for the representation of changeable aspects of the face (Haxby et al., 2000). Moreover, nonvisual neuronal systems, such as those for recognizing emotions, spatial attention, theory of mind and for mirroring actions are involved in the recognition of different facial aspects.

As this thesis focuses primarily on recognizing the emotion shown in a face, the different structures participating in that process will be presented and discussed in detail in the following section. Namely, the most important regions implicated in that process are the occipito-temporal cortices, the amygdala, the orbitofrontal cortex, the anterior cingulate cortex, the somatosensory-related cortices, the basal ganglia, and the insular cortex. Because these structures are engaged in multiple processes and at various points in time, it is difficult to assign a single function to a single structure as outlined in a model by Adolphs (2002a, 2002b) illustrated in Figure 30.

Initial perception of a face modulates activity in subcortical structures as well as in early visual cortices. At this stage the subcortical structures implicated include the superior colliculus and the pulvinar nucleus of the thalamus, which provide a very fast, automatic, and coarse processing of the stimulus and are specialized in analyzing temporally transient signals such as visual motion exhibited by dynamic facial expressions. The information from the pulvinar nucleus of the thalamus is further transferred to the amygdala, while cortical structures such as V1 and V2, and other early visual cortices, receive input from LGN. It has been proposed that the early visual processing might be relatively specialized in extracting information about highly salient stimuli, such as facial expressions of fear and anger, and might be fairly automatic and obligatory. 


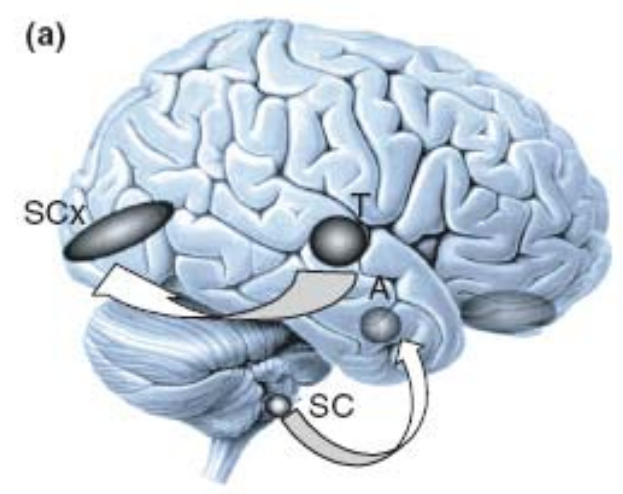

(b)

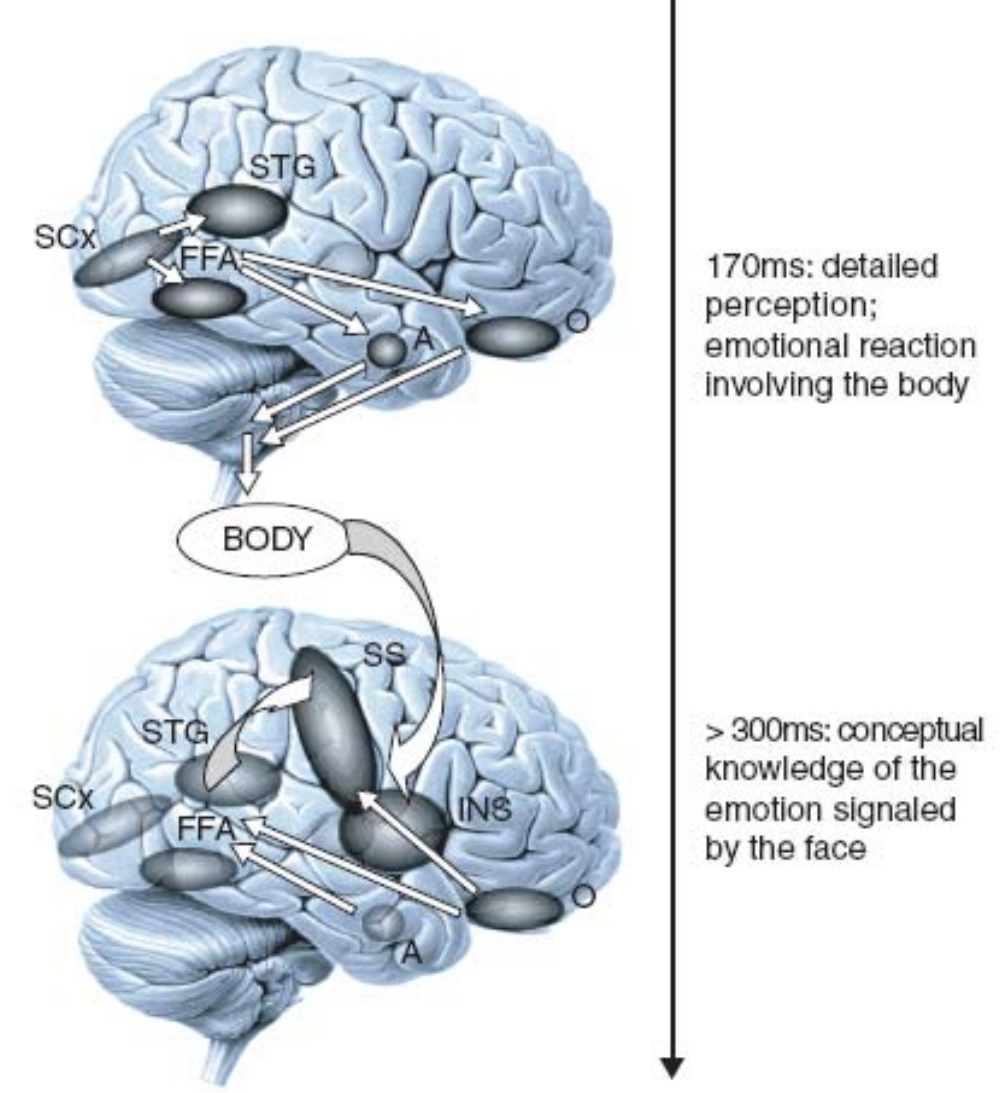

Stimulus onset $\rightarrow$ $120 \mathrm{~ms}$ : fast early perceptual processing of highly salient stimuli

ms: detailed emotional reaction $>300 \mathrm{~ms}$ : conceptual knowledge of the by the face

\section{Figure 30}

Processing of emotional facial expressions as a function of time. (a) Structures involved in emottion recognition at various time points. $\mathrm{A}=$ amygdala; $\mathrm{FFA}=$ fusiform face area; $\mathrm{INS}=$ insula; $\mathrm{O}=$ orbitofrontal cortex; $\mathrm{SC}=$ superior colliculus; $\mathrm{SCx}=$ striate cortex (V1); $\mathrm{SS}=$ somatosensory cortex; STG = superior temporal gyrus; $\mathrm{T}=$ thalamus. (b) Time course of emotion recognition, from the onset of the stimulus at the top, through perception to final recognition of the emotion at the bottom. Certain brain structures are preferentially engaged in processing structural information of the stimulus (early perception), whereas others participate more in retrieving conceptual knowledge or linking the perceptual repesentation to the modulation of other cognitive processes or the elicitation of physiological states (e.g., an emotional somatic reaction to a stimulus) (from Adolphs 2002a).

After early visual cortices have provided a coarse processing of some aspects of the visual stimulus, anterior brain regions including visual association cortices construct a detailed structural representation of the face by about $170 \mathrm{~ms}$ poststimulus onset. The fusiform and 
superior temporal cortices make explicit information useful for recognizing identity and expression, which can be associated with the emotional and social significance of the face. Amygdala and orbitofrontal cortices link the perceptual representation of the facial expression to the conceptual knowledge of the emotion signalled by the face via connections to diverse cortical regions. Importantly, it has to be pointed out that there are feedback influences at multiple temporal scales in this model. Therefore, the attempt to localize the perception and recognition of a face stimulus in space or in time suffers from the fact that the same brain structure participates in different components of processing at different points in time. Thus, an account is needed that describes both the spatial and temporal aspects of processing. In summary, it is important to emphasize that early perceptual processing of the face needs to be linked to many other brain structures to accomplish recognition.

\subsubsection{Visual cortices}

The regions in the occipital and posterior temporal visual cortices play a critical role in the perception and processing of socially and emotionally relevant visual stimuli. Haxby and colleagues (2000) proposed a model of the human neuronal system that mediates face perception thereby differentiating between a core and an extended system (Figure 31).

The core system of face perception consists of mainly three face-responsive regions of occipito-temporal visual extrastriate cortex, which participate differentially in the perception of face identity, face movement and expression. Especially cortical areas in the lateral parts of the inferior occipital gyrus, fusiform gyrus, and superior temporal gyrus are engaged during face processing (McCarthy et al., 1997; Allison et al., 2000; Haxby et al., 2000; 2002) (Figure 32). The anatomical configuration of the face-responsive regions suggests a hierarchical organization in which the inferior occipital gyrus may provide input to the lateral fusiform and superior temporal sulcal regions. The perception of expression, eye gaze direction and speech-related movements shares a common representation of changeable aspects of faces, which are coded in the core system. But not only the changeable aspects of a face, which underlie perception of information that facilitates social communication, are represented in the core system, the invariant aspects, which underlie recognition of unique identity, are part of it as well. 


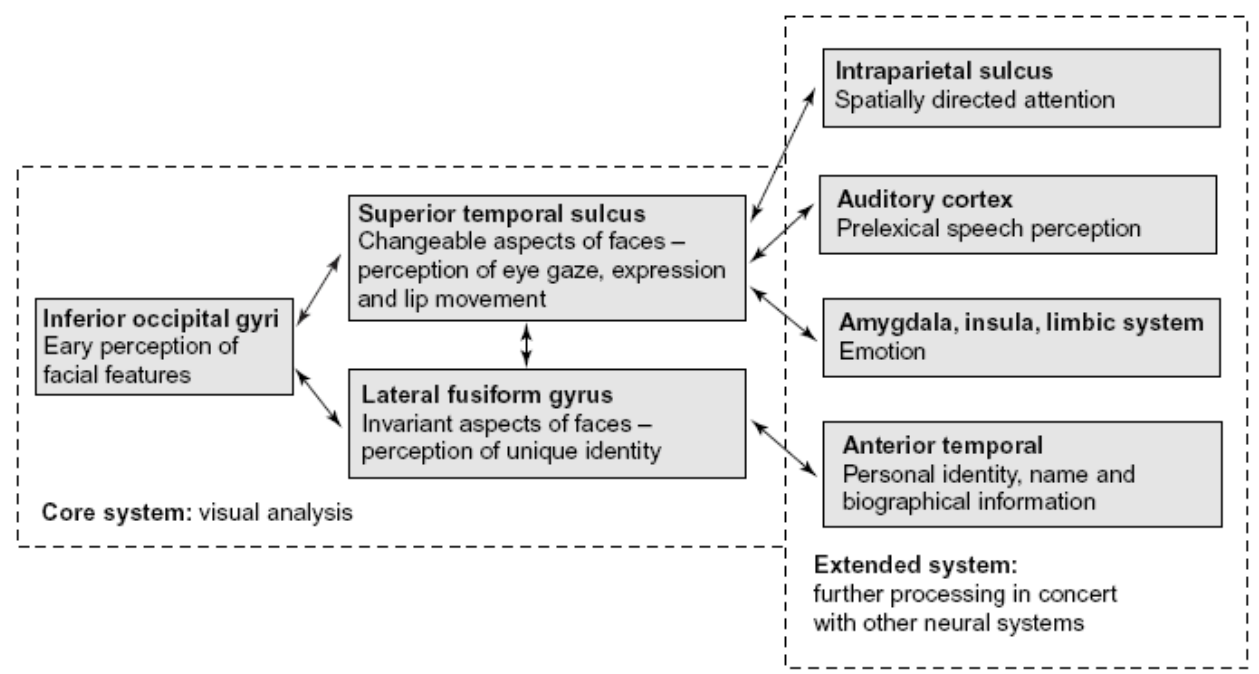

\section{Figure 31}

A model of the distributed human neuronal system for face perception. The model is divided into a core system, consisting of three regions of occipito-temporal visual extrastriate cortex, and an extended system, consisting of regions that are also part of neuronal systems for other cognitive functions. Changeable and invariant aspects of the visual facial configuration have distinct representations in the core system. Interactions between these representations in the core system and regions in the extended system mediate processing of the spatial focus of another's attention, speech-related mouth movements, facial expression and identity (from Haxby et al., 2000).

As the core system provides the visual analysis of faces, the extended system processes the meaning of information gleaned from the face. The extended system comprises additional neuronal systems like the spatial attention system (regions in the intraparietal sulcus and the FEF), which uses facial cues (primarily gaze direction and head position) to direct attention, the emotion processing system (amygdala and insula), which processes the emotional content of expression, systems for auditory verbal comprehension (superior temporal gyrus), which process the phonemic content of speech-related lip movements, as well as systems for representing biographical semantic knowledge for retrieving the name and other information associated with the face (anterior temporal lobe). 


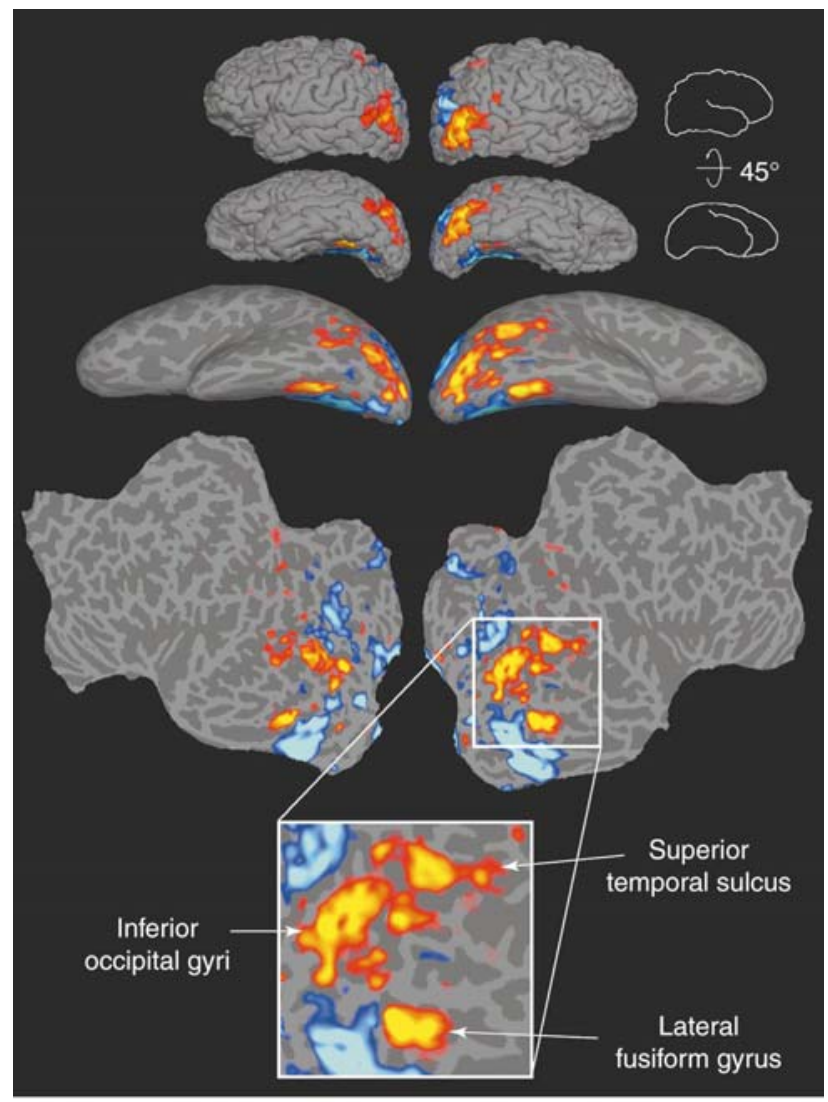

\section{Figure 32}

Cortical regions that comprise the core system for visual analysis of faces from a single subject. Regions shown in red to yellow responded more to faces than to houses. Regions shown in blue responded more to houses than to faces. The upper figures are lateral views of the folded cortical surface. The next row of images shows the cortical surfaces of each hemisphere tilted back $45^{\circ}$ to show both the lateral and ventral surfaces of the temporal lobe. In the next images, the cortical surfaces are inflated to show the cortex in the sulci, indicated by a darker shade of gray. The lower images show the entire cortical surface of each hemisphere flattened into a two-dimensional sheet (from Haxby et al., 2000).

The model proposed by Haxby et al. (2000) shares some elements with an influential cognitive model of face perception by Bruce and Young (1986), which also proposed a distinction between processes involved in the recognition of identity and the recognition of expression and speech-related movements of the mouth. However, the model by Haxby and colleagues (2000) extends that model of cognitive dissociation by underlining the proposal that many face perception functions are accomplished by the coordinated participation of multiple regions represented in the extended system.

The following section gives a brief review of the most relevant neuroimaging studies investigating the face-responsive regions of the core system of face perception, thereby emphasizing the distinction between the perception of structural aspects of the faces that are invariant across changes due to facial movements and the perception of changes due to facial movements. 
The perception of faces has consistently been found to evoke activity in a region in the lateral fusiform gyrus called the FFA (Kanwisher et al., 1997; McCarthy et al., 1997) indicating an apparent selectivity for that category. Although, the face-selectivity of the FFA has been clearly established by numerous studies demonstrating greater activity to faces than to nonsense (control) stimuli or to non-face stimuli (Halgren et al., 1999; Haxby et al., 1994, 1999; Hoffman \& Haxby, 2000; Ishai et al., 1999; Sergent et al., 1992; Puce et al., 1996), other evidence suggests that the submaximal responses in this region to other objects also carry information about the appearance of non-face objects (Chao et al., 1999b; Haxby et al., 2001; Ishai et al., 1999; Ishai, 2000). Indeed, a recent study using high-resolution fMRI showed that the FFA contains a mixture of heterogeneous neuronal populations of voxels responding highly selective to faces as well as to non-face objects (Grill-Spector et al., 2006). Nevertheless, the FFA contains a preponderance of faceselective voxels suggesting a majority of face-selective neurons. However, other studies have indicated that the FFA is not only restricted to face perception, but is also specialized for visual expertise (Gauthier et al., 1999). Studying the expertise for categories of objects other than faces (birds and cars), an increased activity was observed in the FFA in subjects with expertise at recognizing these categories relative to nonexperts (Gauthier et al., 2000), although it is important to mention that the responses evoked by expert viewing of nonface objects were not equivalent to the responses to faces. A recent study by Grill-Spector and colleagues (2004) demonstrated that for most non-face objects (including cars seen by car experts) within-category identification performance was associated with activation in other regions of the ventral occipito-temporal cortex and not in the FFA. These results are in contrast to previous findings showing little involvement of the FFA in within-category indentification of non-face objects. Not only has it been shown that neuronal activation within the fusiform face-selective region is modulated by expertise, beyond that it is also modulated by attention (O'Craven et al., 1999), visual imagery (Ishai et al., 2002; Ishai et al., 2000; O'Craven \& Kanwisher, 2000) and emotion (Vuilleumier et al., 2001). Therefore, it can be assumed that the reponse to faces in extrastriate cortex is not the result of a mere hierarchical, bottom-up process, but is modulated by top-down effects as well. In addition to face-responsive fusiform region, functional imaging studies have identified other face-selective regions located in the lateral inferior occipital gyri and the STS (Haxby et al., 1999; Kanwisher et al., 1997; Halgren et al., 1999; Hoffman \& Haxby, 2000; Puce et al., 1998; Haxby et al., 2002). The inferior occipital region is often adjacent to the lateral fusiform region ventrally and the superior temporal sulcal region dorsally, which lead to 
the assumption that it may provide input to both of these face-responsive regions in temporal cortex (Haxby et al., 1999; Hoffman \& Haxby, 2000).

Numerous functional imaging studies have provided evidence that the posterior STS is activated during the perception of biological movement, including movements of the face, such as eye and mouth movements, and movements of the whole body and hand (Puce et al., 1998; Decety \& Grezes, 1999; Bonda et al., 1996; Allison et al., 2000). Furthermore, the STS is implicated in the perception of still pictures of faces (Chao et al., 1999a; Halgren et al., 1999; Haxby et al., 1999; Hoffman \& Haxby, 2000; Kanwisher et al., 1997), which might reflect involvement in the perception of potential movement or the evaluation of changeable aspects of a face that can vary with movement. This sensitivity of the posterior STS has also been found in the monkey by demonstrating a differential tuning of neurons to the direction of eye gaze, angle of profile, and expression (Hasselmo et al., 1989; Perrett et al, 1984; 1985; 1990; 1992). As the STS has been proposed to code dynamic facial features, it has been shown in single-cell recordings in monkeys (Hasselmo et al., 1989) and humans (Ojemann et al., 1992) as well as human neuropsychological (Rapcsak et al., 1989) and neuroimaging (Gur et al., 1994; Narumoto et al., 2001) studies that this region is also implicated in expression processing. A recent study by Engell and Haxby (2007) revealed that the response to faces in the right STS is significantly modulated by both the perception of expression and by the perception of averted-gaze. However, an analysis within that region indicated that gaze-direction and expression are coded by distinct, though overlapping, neuronal populations in the right STS. Moreover, Calder et al. (2007) provided first evidence of dissociable neuronal systems for left and right gaze represented in the right anterior STS in humans.

As mentioned earlier, the core system differentiates between the functional roles played by the face-responsive regions STS and FFA. Hoffman and Haxby (2000) directly tested this dissociation by measuring how selective attention to eye gaze direction and identity differentially modulates the responses to faces in these regions. As predicted, selective attention to eye gaze induced stronger responses in the STS whereas attention to identity was associated with increased activation in the FFA. Another fMRI-study by Winston and colleagues (2004) broadly supports this model in demonstrating adaptation in the fusiform cortex when the identity of a face and in the STS when the emotional expression of a face is repeated. These results provide evidence for the double dissociation between the functional contributions of the STS and the lateral fusiform gyrus to face perception. 


\subsubsection{Amygdala}

The amygdala is essential for several domains of emotional behaviour (Zald 2003), such as fear conditioning (Blair et al., 2005), emotional memory (Adolphs et al., 2005), mood induction (Habel et al., 2005) and emotion discrimination (Gur et al., 2002). The amygdaloid complex, is a collection of morphologically, histochemically, and functionally diverse nuclei. Located largely within the rostral temporal lobe, the main portion of the amygdala is almond-shaped (amygdala is Greek for 'almond'). It comprises the basal, central, lateral, and superficial groups of nuclei. In addition to the afferent and efferent connections, there are several intrinsic connections between the amygdaloid nuclei. Based on function and connections the amygdaloid complex is commonly divided into three groups: the basolateral, corticomedial and central nuclei (Figure 33).

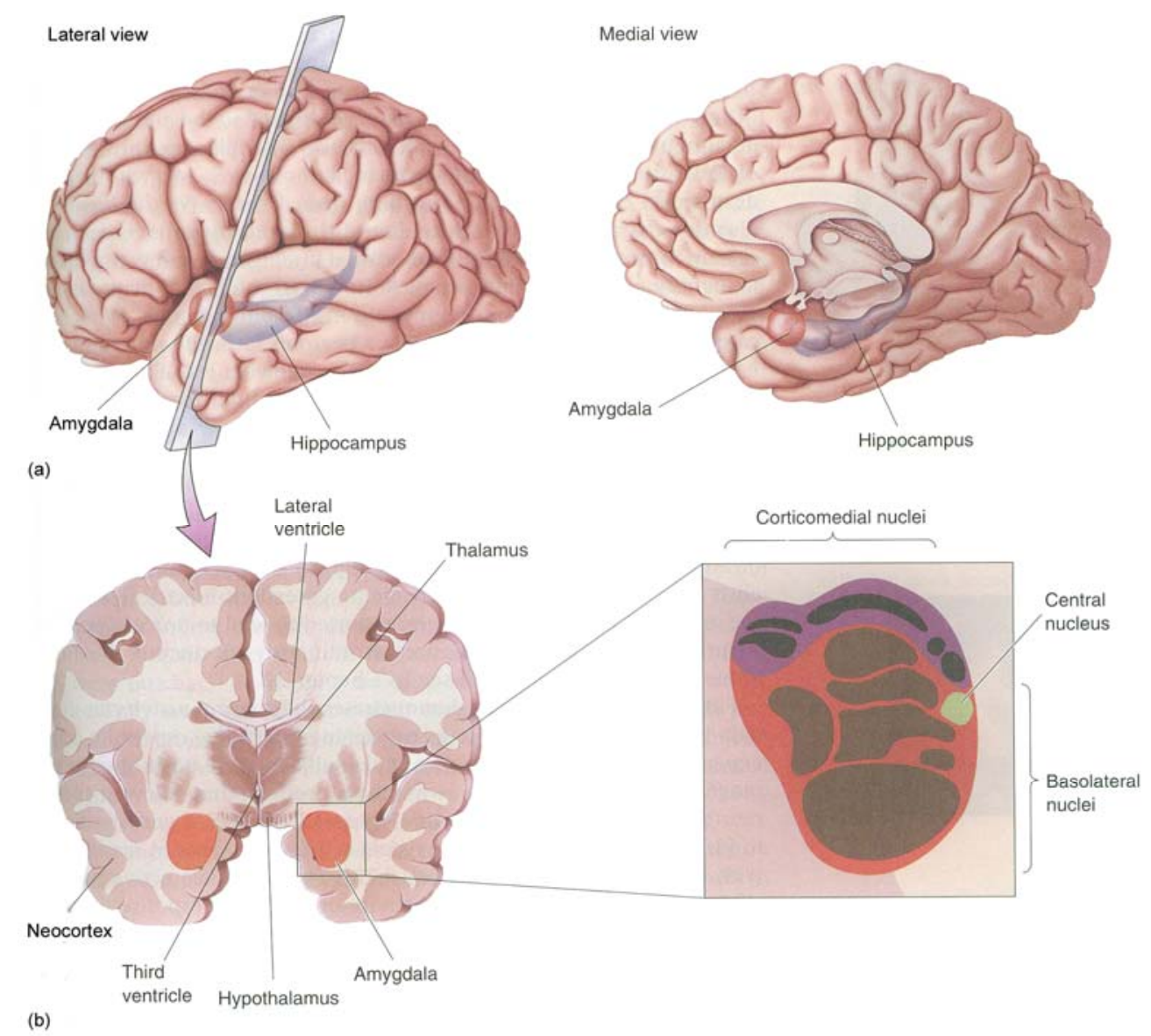

Figure 33

A cross section of the amygdala. (a) Lateral and medial views of the temporal lobe, showing the location of the amygdala in relation to the hippocampus. (b) The brain is sectioned coronally to show the amygdala. The basolateral nuclei (surrounded by red) receive visual, auditory, gustatory, and tactile afferents. The corticomedial nuclei (surrounded by purple) receive olfactory afferents (from Bear et al., 2007). 
The basolateral nuclei comprise the largest division of the amygdala. These nuclei are thought to attach emotional significance to a stimulus. The basolateral nuclei receive information about the particular characteristics of a stimulus from higher-order sensory cortical areas in the temporal and insular cortical areas and from association cortex. The major efferent connections are directed back to the cerebral cortex, either directly or indirectly. The cortical areas receiving a direct projection from the basolateral amygdala are the limbic association cortex (cingulate gyrus, temporal pole, medial orbitofrontal cortex) and the prefrontal cortex. The amygdala also projects directly to the hippocampal formation, which is thought to be important in learning the emotional significance of complex stimuli or the context in which emotionally charged stimuli are experienced. In addition to direct cortical projections, the basolateral division has extensive subcortical projections that give rise, indirectly, to connections to the cortex. The basolateral nuclear group has connections with the medial dorsal nucleus of the thalamus (via the ventral amygdalofugal pathway), the basal nucleus and the ventral striatum (Figure 34, red). The corticomedial nuclei are mainly connected with the hypothalamus, central visceral nuclei, and the olfactory bulb (Figure 34, purple). The central nuclei mediate emotional responses. In regulating the automatic nervous system, the central nuclei receive input from brain stem nuclei. In turn, the central nuclei project via the ventral amygdalofugal pathway to autonomic nuclei in the brain stem. The central nuclei also regulate the autonomic nervous system through projections to the lateral hypothalamus. Furthermore the central nuclei receive an input from the basolateral nuclei which constitutes the key path for fear conditioning and helps to shape responses to emotional stimuli (Figure 34, green). 


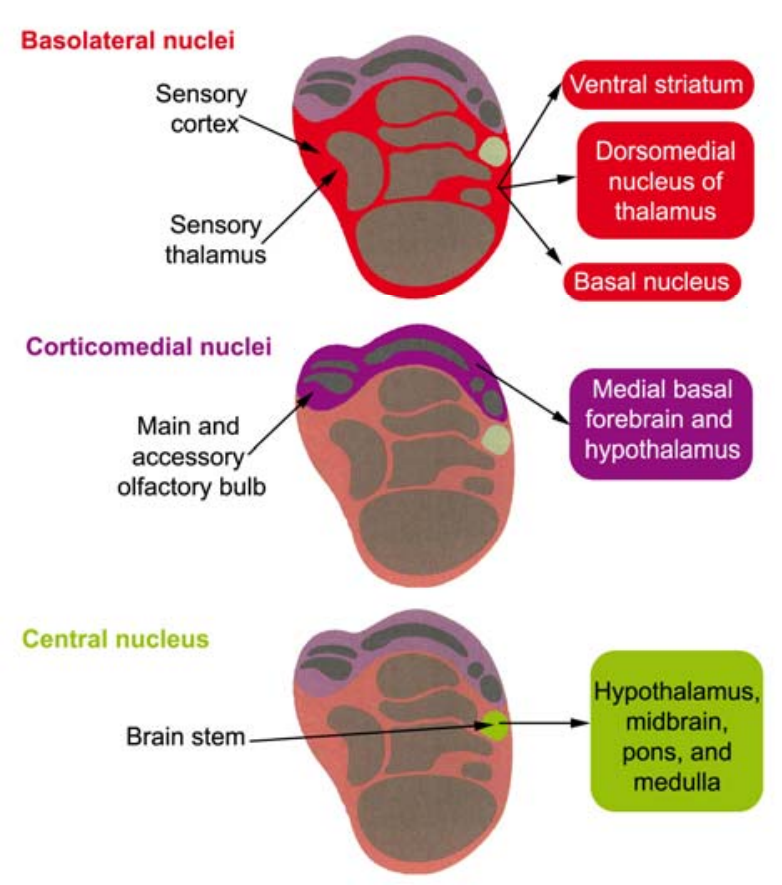

\section{Figure 34}

Diagram of the major divisions and connections of the amygdala

A large corpus of research at the animal level has established the importance of the amygdala for emotional processes (Aggleton, 1993; Cahill \& McGaugh, 1998; LeDoux, 1996). LeDoux and his colleagues have provided compelling evidence that the amygdala is necessary for the establishment of conditioned fear and promoted the idea that the amygdala participates in the recognition of emotional signals via at least two classes of input mechanisms: a subcortical route (low road) via the superior colliculus and the pulvinar nucleus of the thalamus, and a cortical route (high road) via the visual neocortex (Figure 35).

It has been suggested that the position of the amygdala and its connectivity allows it to function as a crude though rapid registry of the emotional value of incoming stimuli. LeDoux described this first evaluation of emotional stimuli that are processed via the subcortical route as quick and dirty and termed it affective computation. Furthermore, the amygdala is proposed to encode the emotional value before or even without the need for the full-blown object-level perception of the stimuli arising in higher-level associative cortices that is required for conscious awareness of the stimuli to ensue. The cognitive computation to evaluate the emotional significance of a stimulus via the cortical route takes longer, but supplies the amygdala with details on the stimulus to enable a precise classification and differentiation in order to either further or obstruct a person's goals. 


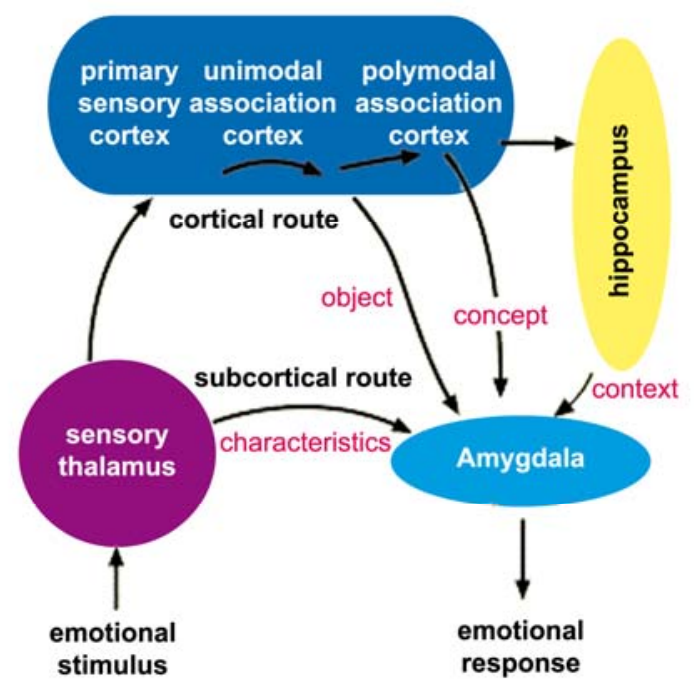

\section{Figure 35}

Schematic illustration of the two parallel input routes to the amygdala: the thalamo-amygdala pathway (subcortical or short route) and the thalamo-cortico-amygdala pathway (cortical or long route) (adapted from www.thebrain.mcgill.ca). The short route conveys a fast, rough information of the incoming stimuli. This pathway activates the central nucleus of the amygdala and generates an emotional response before any perceptual integration and a complete representation of the stimulus has even occurred. Subsequently, stimulus information following the long route is first processed by the primary sensory cortex. Then the unimodal associative cortex provides the amygdala with a representation of the object. The polymodal associative cortex conceptualizes the object and passes on the information to the amygdala. This elaborate representation of the object is then compared with contents of explicit memory by means of the hippocampus.

A number of neuroimaging studies have reported activation of the amygdala in response to facial expressions of fear compared with neutral, happy, or disgust control faces (Breiter et al., 1996a; Morris et al., 1996a; Phillips et al., 1997; Yang et al., 2002; Gorno-Tempini et al., 2001; Iidaka et al., 2001; Phillips et al., 1998a; Phillips et al., 2004; Schneider et al., 1997; Wright et al., 2002). However, not only fearful faces activate the amygdala, the presentation of angry (Nomura et al., 2004; Sato et al., 2004; Yang et al., 2002; Fitzgerald et al., 2006), disgusted (Schienle et al., 2002; Fitzgerald et al., 2006), sad (Blair et al., 1999; Yang et al., 2002; Fitzgerald et al., 2006) or even neutral faces (Kesler-West et al., 2001; Fitzgerald et al., 2006; Wright \& Liu, 2006) revealed amygdala activation as well. Increased activity in the amygdala has also emerged when faces of people who are viewed in negative ways, such as being untrustworthy, bizarre looking or from a negatively viewed ethnic group, were presented (Phelps et al., 2000; Rotshtein et al., 2001; Winston et al., 2002). Additionally, some studies provide examples in which happy faces produce an increase in amygdala activity (Wright et al., 2002; Killgore \& Yurgelun-Todd, 2001b; 
Gorno-Tempini et al., 2001; Breiter et al., 1996a; Fitzgerald et al., 2006), but on the other hand some report activity decreases (Morris et al., 1996a; Whalen et al., 1998).

A growing body of studies from a small group of human patients with discrete lesions in the amygdala highlight the importance of this region for both the perception and production of negative affect and associative aversive learning. Amygdala lesions in humans result in emotional blunting (Aggleton \& Brown, 1999) and reduced fear conditioning (Bechara et al., 1995). Adolphs and his colleagues (1994) reported that a patient with selective amygdala lesions was impaired in recognizing fear in facial expressions while recognition of other facial expressions was intact. Furthermore, the patient was able to determine the personal identity from faces. These findings suggest that damage restricted to the amygdala causes very specific recognition impairments. Thus, patients with unilateral amygdala damage were not impaired at recognizing any facial expressions (Adolphs et al., 1995). Two additional studies using a different methodology for the assessment of facial expression recognition also found a specific deficit in the recognition of facial expressions of fear in patients with amygdala damage (Calder et al., 1996; Broks et al., 1998). However, it is likely that the deficit in the perception of expressive signs of fear in patients with amygdala damage is not specific to facial expressions since deficit in the recognition of vocalic expressions of fear and anger has been demonstrated in these patients as well (Scott et al., 1997). Moreover, Anderson and Phelps (2001) showed that lesions of the amygdala disrupt its ability to modulate the efficiency of word processing and therefore the fluency of perceptual encoding. The importance of the amygdala in processing information about facial emotions has been demonstrated by a number of lesion studies (Young et al., 1995; Adolphs et al., 1999; Schmolck \& Squire, 2001; Adolphs et al., 1998b; Vuilleumier et al., 2004). An explanation for the lack of recognizing fear in faces in patients with amygdala damage is provided by Adolphs and colleagues (2005) who proposed that these patients suffer from an inability to make use of information from the eye region of faces when judging emotions during free viewing. The impairment in recognizing fear is explained by the fact that the eyes are the most important feature for identifying this emotion. However, it has to be taken into account that the patient's recognition of fearful faces improved when she was instructed explicitly to look at the eyes. The amygdala is known to participate in processing information about the eye region of faces (Kawashima et al., 1999; Morris et al., 2002) and recently, a neuroimaging study in monkeys has demonstrated that facial expressions and gaze/head orientation engage distinct sections of the amygdala (Hoffman et al., 2007). 
Such a functional specialization might account for the role of the amygdala in processing emotions related to behavioural withdrawal, fear, threat or danger (Anderson et al., 2000; Calder et al., 1996; Adolphs et al., 1999).

Next to the emotional valence there are several other known neuromodulatory factors influencing the activity in the amygdala such as gender, arousal, anxiety, task demands, handedness and emotion regulation, just to mention some of them. To describe all of them would go beyond the scope of this thesis and can be found elsewhere (Zald 2003; Wager et al., 2003).

Several neuroimaging studies suggest that gender differences influence the extent and/or laterality of amygdala responses in human subjects. Gender differences are often reported in regard to hemispheric lateralization of several structures and functions including the amygdala. These sex differences in lateralization of amygdala responses have also emerged in studies involving the viewing of emotional facial expressions (Killgore \& YurgelunTodd, 2001a; 2001b; Schneider et al., 2000; Thomas et al., 2001; Kesler-West et al., 2001; Lee et al., 2002; McClure et al., 2004). However, the findings of these studies present a contradictory picture. For example Kesler-West and colleagues (2001) reported greater activation in the right hemisphere of men during angry face perception whereas responses to sad faces were left lateralized. In women no such differences between the valence of emotions in either hemisphere were observed. On the contrary, McClure and colleagues (2004) found an increase of activation in the amygdala elicited by angry versus fearful and angry versus neutral faces to be right lateralized in women whereas another study demonstrated a left lateralization of amygdala activity induced by happy faces for men only and left lateralization for fearful faces in both sexes (Killgore \& Yurgelun-Todd, 2001b). Nevertheless, in a recent meta-analysis of emotion induction and pleasant and unpleasant visual and auditory stimulation studies, Wager and colleagues (2003) observed a similar pattern of lateralization of amygdala findings: There was a trend toward greater left rather than right lateralized amygdala activation in both genders. Although it is important to take gender differences into account when emotional paradigms are tested with fMRI, the gender effects are likely to be relatively specific to the domains in question, rather than reflecting a more global difference in the lateralization of amygdala responses to all emotional stimuli.

Studies investigating to which extent different task demands and instructions influence amygdala responses to emotionally salient stimuli revealed inconsistent results. Some studies indicate a difference between explicit and implicit coding (Gur et al., 2002; Habel 
et al., 2007), while others observed no effect of explicit versus implicit processing of emotional faces (Gorno-Tempini et al., 2001; Rotshtein et al., 2001), or even greater activity during implicit relative to explicit conditions (Critchley et al., 2000). Another related issue deals with the question whether the encoding of emotion is resourcedependent or not. In fact, only a few fMRI studies to date have examined whether the processing of emotional stimuli places demands on attentional resources. Data on the interaction between emotion and attention have presented a mixed picture and remain contentious.

The most relevant influencing aspects regarding the activation of the amygdala for this work such as target visibility, attentional load as well as the location, saliency and spatial frequency of emotional stimuli will be discussed in detail in the next chapter.

In summary, the findings from both the lesion and neuroimaging studies clearly implicate the amygdala as an important site for the control of emotion in the human brain. Activation in the amygdala is reliably produced by the presentation of biologically-relevant sensory stimuli, even in the absence of cortical processing and visual awareness, thereby participating during the initial phase of stimulus evaluation. However, the published results concerning the functionality of the amygdala are extremely heterogenous for several influencing factors e.g. valence, gender and task demands.

\subsubsection{Other brain regions}

Additional to the described brain areas above there are several other structures that are important for emotion recognition in humans: the prefrontal cortex, the anterior cingulate cortex, the right somatosensory-related cortices, the insula, and basal ganglia. All these structures are indispensable components of a neuronal system for retrieving knowledge about the emotions signalled by facial expressions.

\subsubsection{Prefrontal cortex}

An additional key structure in emotion processing, especially important for the recognition of faces, with which the amygdala is intimately connected, is the orbitofrontal cortex. The orbitofrontal cortex is part of the PFC, which consists of several important subdivisions that are critical to affective processing. The PFC is separated into the dorsolateral 
(DLPFC), ventromedial (VMPFC) and orbitofrontal (OFC) cortex. Within these regions the left and right sectors are distinguished from each other (Figure 36).
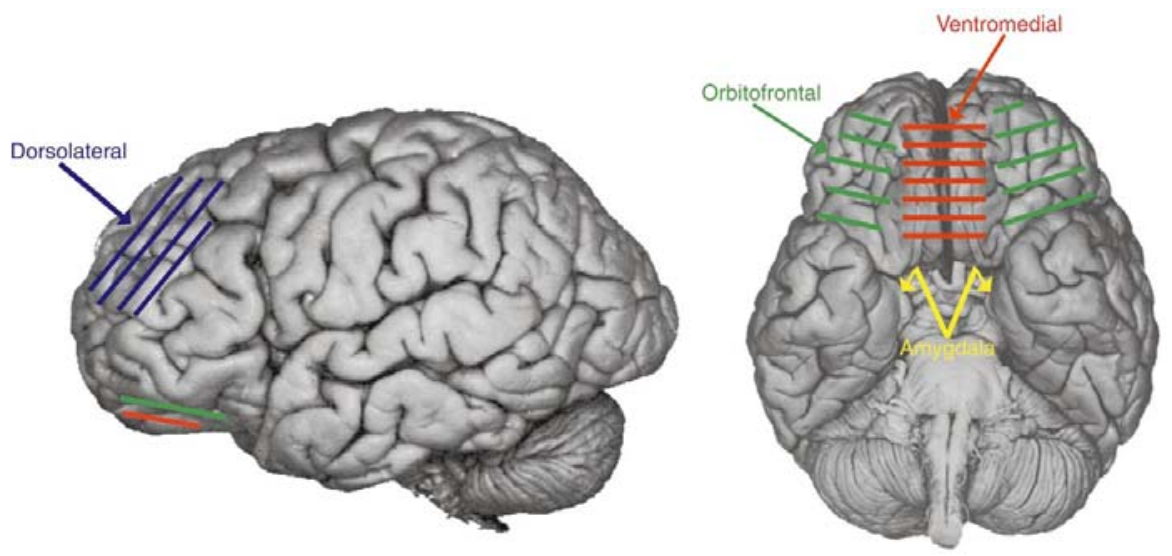

\section{Figure 36}

Sectores of human prefrontal cortex. The figure shows dorsolateral (blue), ventromedial (red) and orbitofrontal (green) cortical territories in a lateral view (left image) and in a ventral view (right image) (from Davidson \& Irwin, 1999).

The differential involvement of the PFC in certain forms of positive and negative emotion is supported by several studies (Davidson et al., 1990; Davidson, 1992; 1998; Sutton et al., 1997; Sutton \& Davidson, 1997; Lane et al., 1997; Rauch et al., 1997; Breiter et al., 1996b; Kawasaki et al., 2005) indicating a right-sided lateralization for negative emotions. Furthermore, neuroanatomical studies of the limbic forebrain have identified two parallel pathyways by which emotionally arousing stimuli processed in the amygdala potentially interface with PFC (Mega et al, 1997). The first pathway is the canonical medial circuit linking the basal amygdala with ventromedial OFC (BA 11), rostral insula, and subgenual portions of the anterior cingulate cortex (ACC) (BA 25). A second lateral pathway interconnects inferotemporal cortex and basal amygdala with ventrolateral PFC (BA 10/47) and rostral ACC (BA 24/32). The left DLPFC has been shown to contribute in certain features of positive affect, which, when disrupted, increases the probability of depressive symptomatology (Morris et al., 1996b; Robinson \& Downhill, 1995). The VMPFC is involved in the anticipation of future positive and negative consequences of actions (Bechara et al., 1994; 1996; 1998). The most relevant prefrontal region in the context of this work is the OFC as it has been demonstrated to be involved in the recognition of facial expressions (Hornak et al., 1996) and identity (Ishai et al., 2005).

Prefrontal and temporal cortices have been shown to have an intimate reciprocal connectivity necessary for normal processing of visual stimuli and the knowledge previously associated with them (Fuster et al., 1985; Tomita et al., 1999). Recent evidence 
indicates that the PFC can respond rapidly to faces and facial expressions (Kawasaki et al., 2001; Marinkovic et al., 2000). The extensive connections between temporal visual regions and PFC provide the perceptual input to OFC which therefore could play a role in recognizing facial emotion via direct modulation of activity in temporal cortex via feedback. Based on the close anatomical and functional relationship between amygdala and OFC, it has been stated that the PFC might provide contextual modulation of the amygdala's processing of social facial signals (Emery \& Amaral, 1999), and furthermore modulates a large variety of basic emotional responses mediated by the amygdala (Jackson \& Moghaddam, 2001; Kalin et al., 2001; Quirk et al., 2000).

First evidence for a participation of the OFC in processing emotions in faces was provided by a lesion study by Hornak and colleagues (1996) reporting impaired recognition of emotion from facial expressions following damage to this region. To further determine the details of the emotion categories activating the OFC functional imaging methods were used. The left inferior frontal lobe, including the orbitofrontal region, was found to be activated during the processing of all basic emotions supporting the idea that the output of different emotion systems related to fear, anger and disgust converge on frontal regions for further information processing (Sprengelmeyer et al., 1998). Other imaging studies revealed an activation in the orbitofrontal region to fearful compared to neutral faces (Vuilleumier et al., 2001; Kesler-West et al., 2001), angry but not sad faces (Blair et al., 1999) and neutral facial stimuli as well (Morris et al., 1998). Moreover, increased activation in the OFC was observed when subjects were engaged in a cognitive task requiring explicit identification of facial emotion (Nakamura et al., 1999; Narumoto et al., 2000; Blair et al., 1999), but also when subjects had not to perform any explicit task (passive viewing) (Ishai et al., 2005). Some intriguing data come from single-unit recordings from patients who had depth electrodes implanted for the purpose of monitoring seizures. Single-unit responses were obtained in right ventromedial PFC in response to presentation of facial expressions of fear and happiness indicating a first neuronal discrimination between the two emotions after 120 ms (Kawasaki et al., 2001).

Overall, there is clear evidence for the orbitofrontal region in processing emotional information from faces and in exhibiting rapid responses to emotionally salient stimuli. The OFC is therefore implicated in the mediation of autonomic changes accompanying affective states produced in response to emotional stimuli or contexts suggesting that this region plays an important role in the automatic regulation of emotional behaviour. 


\subsubsection{Anterior cingulate cortex}

The ACC is a large medial cerebral structure surrounding the anterior third of the corpus callosum. On the basis of cytoarchitecture, patterns of projections, and of function the ACC can be differentiated from the posterior cingulate cortex. So far, a variety of different observations have been interpreted to favour a specialization of ACC subdivisions for cognitive versus emotional processing. Therefore, the ACC has been divided into two major distinct sub-territories (Figure 20): the dorsal cognitive division (dACC) and the rostral-ventral affective division (rACC) (Bush et al., 2000; Devinsky et al., 1995). The cognitive division has been activated by cognitively demanding tasks that involve stimulus-response selection in the face of competing streams of information, including Colour Stroop and Stroop-like tasks, divided-attention tasks, verbal- and motor-response selection tasks and many working memory tasks (Bush et al., 2000). In contrast the affective division has been activated by affect-related tasks as well as by mood-inducing tasks (Bush et al., 2000).

The cognitive division of the ACC comprises caudal and dorsal regions, including BA 24b'-c' and 32' (Figure 37) and is extensively connected with PFC, in particular with the DLPFC as well as the primary, premotor, and supplementary motor areas (Barbas \& Pandya, 1989; Paus, 2001, Luppino et al., 2003). It has been proposed that dACC plays a critical role in attention-demanding executive control processes (Posner, 1995; Posner \& Petersen, 1999; Frith, 2001). Moreover, dACC has been demonstrated to be specifically engaged in processing conflict between competing stimuli or responses (Banich et al., 2000; Carter et al., 1998; Kerns et al., 2004; MacDonald et al., 2000). Detection of response conflict is important in monitoring performance (Botvinick et al., 2001; 2004), and neuroimaging data show dACC sensitivity to errors in performance (Kiehl et al., 2000; Menon et al., 2001; Critchley et al., 2005). Furthermore, a functional differentiation within dACC has been proposed (Milham \& Banich, 2005), with more posterior portions showing involvement in late-stage attentional processes, which are usually response-related (Milham et la., 2001; Milham et al., 2003), and anterior portions involved in response evaluation (Milham \& Banich, 2005). 


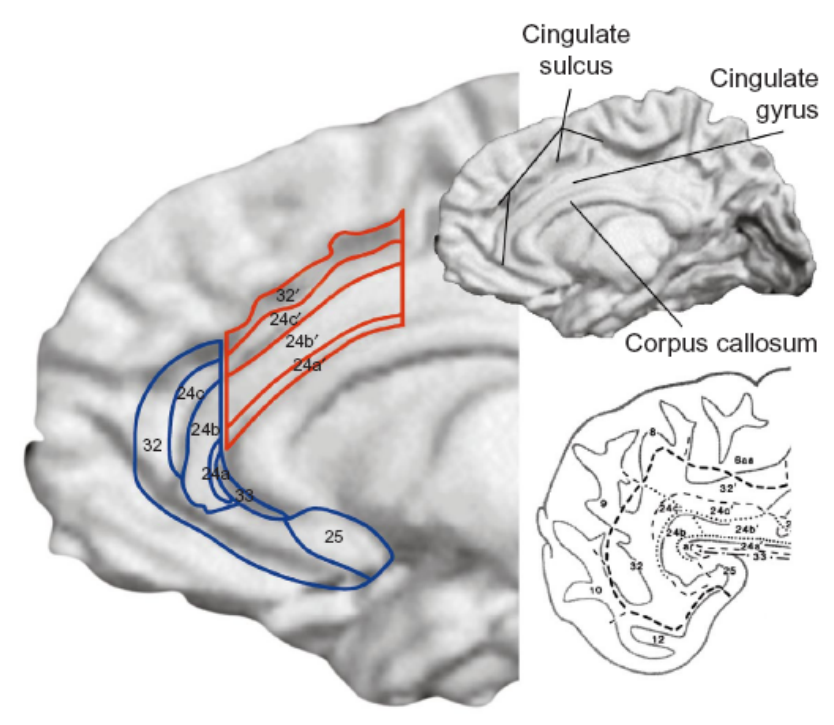

\section{Figure 37}

Anterior cingulate cortex (ACC) anatomy. The upper right part of the figure contains a reconstructed MRI of the medial surface of the right hemisphere of a single human brain (anterior towards the left, posterior towards the right). The cortical surface has been 'partially inflated' to allow simultaneous viewing of gyri and sulci. In this example, a single cingulate gyrus lies between the cingulate sulcus and the corpus callosum. A schematic representation of cytoarchitectural areas (numbered) of ACC is shown on the enlarged section (left). Cognitive division areas are outlined in red and affective division areas are outlined in blue. A schematized flat map of actual anterior cingulate cortical areas is shown in the bottom right panel. The borders of each sulcus appear as thin unbroken black lines, whereas a combination of broken and dotted lines outline cingulate areas (from Bush et al., 2000).

The affective division is a ventral region of the ACC comprising subgenual anterior cingulate gyrus (BA 25), BA 32, and pregenual anterior cingulate gyrus (rostral regions of BA 24) (Figure 37). This part of the ACC has been consistently associated with the assessment of emotional information as well as the regulation of emotional responses (Devinsky et al., 1995; Paus, 2001; Drevets \& Raichle, 1998; Whalen et al., 1998). It has extensive connections with the amygdala, periaqueductal gray, mediodorsal and anterior thalamic nuclei, nucleus accumbens, ventral striatum, anterior insula, hippocampus and OFC (Öngür \& Price, 2000; Barbas et al., 2003; Devinsky et al., 1995), and has outflow to autonomic, visceromotor and endocrine systems. rACC has been shown to be implicated in emotional processing in normal individuals and in symptom provocation in anxiety disorders (Bush et al., 2000; Devinsky et al., 1995; Drevets \& Raichle, 1998; Whalen et al., 1998; Ochsner et al., 2002). Rather than being specialized distinctly for emotion, rACC plays a critical role in tasks requiring cognitive control in the presence of emotional stimuli (e.g. faces). Cognitive control is especially important when task-irrelevant emotional stimuli interfere with task-relevant processing. Several studies provided evidence for the involvement of $\mathrm{rACC}$ in the implementation of this control and evaluation of interference 
from emotionally salient but task-irrelevant stimuli (Bishop et al., 2004; Vuilleumier et al., 2001; Whalen et al., 1998). rACC has been further implicated in monitoring of errors (Kiehl et al., 2000).

Although studies indicate the differential role of dACC and $\mathrm{AACC}$ in cognitive and emotional function (Bush et al., 2000), respectively, evidence suggests that the functional implications of these two regions are not so distinct. It has been demonstrated that increases in rACC activity are associated with decreased amygdala activity, but both dACC and rACC negatively modulate the thalamus-amygdala relationship (Das et al., 2005; Ochsner et al., 2002; Petrovic et al., 2004). On the other side, there is also evidence for dACC involvement in emotional processing. Attention-demanding tasks involving emotional content can alter the firing rate of dACC neurons (Davis et al., 2005), and emotional processing appears to foster increased dACC activity particularly in the context of concomitant cognitive demands (Phan et al., 2002). Therefore, it has been suggested that the involvement of $\mathrm{dACC}$ in cognitive as well as emotional processes might reflect its more general role in the modulation of autonomic responses related to adaptive behavioural control (Critchley et al., 2003). However, a recent study provided direct evidence for the differential engagement of ACC subdivisions in cognitive and emotional processing and for differential functional connectivity in the implementation of cognitive control and emotion regulation (Mohanty et al., 2007).

\subsubsection{Somatosensory-related cortices}

The finding that somatosensory regions are critical for the processing of emotional information has been mainly supported by lesion studies indicating that lesions in the right ventral primary and secondary somatosensory cortex were systematically and significantly associated with impaired recognition of emotion from facial expressions (Adolphs et al., 1996; Adolphs et al., 2000). According to Adolphs and colleagues (2000) these results can be explained by the idea that emotional facial expressions can also be thought of as facial actions and as such would be expected to draw on some of the same simulation mechanisms found for action perception in general. Thus, viewing facial expressions of emotion triggers an emotional response in the perceiver that mirrors the emotion shown in the stimulus (Wild \& Bartels, 2001), and representing this emotional response in somatosensory cortices in turn provides information about the emotion. This hypothesis, that knowledge of other people's emotions might be based on simulating the observed 
emotion, is in accordance with several other studies in humans (Hari et al., 1998; Strafella \& Paus, 2000; Iacoboni et al., 1999; Buccino et al., 2001; Iacoboni, 2005) and monkeys (Rizzolatti \& Luppino, 2001; Rizzolatti et al., 2002; Gallese et al., 1996; Rizzolatti et al., 1996; Rizzolatti et al., 1999). The role for somatosensory-related cortices in emotion recognition might also be related to the finding that damage to those regions can result in anosognosia, an impaired knowledge of one's own body state which is often accompanied by a flattenting of emotion (Damasio, 1995). However, it remains unclear which components of the somatosensory cortex are most important for the recognition of emotion, those regions that represent the face or those that provide more comprehensive knowledge about the state of the entire body associated with an emotion. In the study by Adolphs and colleagues (2000) a disproportionate role for the face representation within $\mathrm{S} 1$, but also additional somatic representations in S2, insula, and the anterior supramarginal gyrus has been suggested. Nevertheless, to date there are no functional imaging studies directly supporting the idea that somatosensory cortices play a role in the recognition of facial emotion in virtue of their ability to help construct a somatosensory image of the body state associated with this emotion.

\subsubsection{Insular cortex and basal ganglia}

The insular cortex is buried deeply in the lateral fissure. Its borders are defined by the frontal, parietal and temporal cortex. The rostral end of the insula is a poorly understood part of the limbic system and the caudal end of the insula is involved in somatosensory processing. The fact that a very diverse range of manipulations produce activation in the insular cortex is consistent with the idea that this structure plays a critical role in visceral representation. The insular cortex receives afferents from several major autonomic regions and sends efferents to a number of brain regions that play a critical role in regulating autonomic responses that accompany emotion including the central nucleus of the amygdala and the lateral hypothalamus. The insular cortex is topographically organized and includes a major zone dedicated to gustatory processing.

Activation of the insula has been reported in a wide range of different neuroimaging studies manipulating emotion (Lane et al., 1997; Breiter et al., 1997; Rauch et al., 1995). As the sensory representation for taste is located in the insular cortex, this fact might be responsible for the significant insular activation detected with fMRI in response to faces depicting disgust, which can be understood as signifying distasteful stimuli (Phillips et al., 
1997; Calder et al., 2001; Phillips et al., 1998a; Sprengelmeyer et al., 1998; Schienle et al., 2002). These findings have been completed by lesion studies reporting pronounced impairments both in the ability to recognize facial expressions of disgust as well as in the ability to experience the emotion himself following damage to left insula and basal ganglia (Calder et al., 2000) and in the ability to recognize and experience disgust from all types of stimuli after bilateral damage to insula in addition to extensive temporal lobe damage (Adolphs et al., 2003).

The term 'basal ganglia' refers to five subcortical nuclei situated bilaterally in the white matter of the cerebral hemispheres. These nerve cell nuclei are the caudate nucleus, putamen, subthalamic nucleus, substantia nigra, and the globus pallidus. The basal ganglia receive afferents from the cerebral cortex, the intralaminar nuclei of the thalamus, and dopaminergic pathways from the midbrain and send major efferent inputs to the cortex via the thalamus.

Especially neuroimaging studies inducing happiness have shown an involvement of the basal ganglia in the perception, recognition and processing of emotion (Breiter et al., 1997; Koch et al., 1996; Morris et al., 1996a, 1998; Phillips et al., 1998b; Lane et al., 1997, Lane et al., 1999; Davidson \& Irwin, 1999; George et al., 1996; Damasio et la., 2000). Given its rich innervations of mesolimbic dopaminergic neurons, the basal ganglia and ventral striatum are well positioned to respond to incentive reward motivation and pregoal attainment of positive affect arising from progression toward a desired goal, consistent with the notion that happiness can be conceptualized as an approach emotion (Davidson \& Irwin, 1999; Davidson et al., 1990). However, the basal ganglia are not only linked to positive emotions, but also to withdrawal emotions like disgust. Particularly, facial expressions of disgust activated the basal ganglia (Phillips et al., 1997, 1998a; Sprengelmeyer et al., 1998). Additionally, diseases that preferentially damage certain sectors of the basal ganglia offer special insight into the role of the basal ganglia in emotion recognition like obsessive-compulsive disorder, Parkinson's disease and Huntington's disease. Indeed, impaired recognition of facial expressions of disgust has been reported in patients with obsessive-compulsive disorder (Sprengelmeyer et al., 1997) and Huntington's disease patients (Suzuki et al., 2006; Sprengelmeyer et al., 1996; Gray et al., 1997; Jacobs et al., 1995a), although a recent study extended these findings by demonstrating impaired recognition of different negative emotions prior to diagnosis in Huntington's disease (Johnson et al., 2007). However, the findings regarding Pakinson's 
disease are inconsistent: Some studies have reported impaired recognition of facial emotion in this disease (Blonder et al., 1989; Jacobs et al., 1995b; Pell \& Leonard, 2005; Lachenal-Chevallet et al., 2006), whereas others failed to find such an impairment (Adolphs et al., 1998a).

\subsection{Processing of emotional stimuli}

The traditional perspective of differentiating the affective and cognitive system relates to the question whether the emotional value of stimuli can be encoded 'automatically' (preattentively) or whether it is processed in a controlled manner by referring to the dependence on attentional resources (Pashler, 1998). According to this conception, some mental processes place minimal demands on attentional resources, and such processes have been labelled 'automatic'. Automatic processes are carried out independently of available resources and are therefore relatively immune to disruption or interference from other ongoing operations. Furthermore these processes are very rapid, non-conscious and mandatory. In contrast, controlled processes are conscious, demand some measure of attentional resources and can be disrupted by competing operations or tasks.

\subsubsection{Automatic processing}

Automatic processing of emotional stimuli is supposed to be fast. In terms of face recognition psychophysiological studies using ERPs and MEG demonstrated that emotional information from faces is registered and discriminated, from as early as $80 \mathrm{~ms}$ after stimulus onset in occipital regions (Pizzagalli et al., 1999; Eger et al., 2003; Pourtois et al., 2004; Halgren et al., 2000) and 100-200 ms after stimulus onset in the amygdala (Streit et al., 2003; Liu et al., 1999; Halgren et al., 1994; Streit et al., 1999; Krolak-Salmon et al., 2004). In frontal and temporal regions it takes between 100 and $250 \mathrm{~ms}$ to process faces (Liu et al., 1999; Streit et al., 1999; Pizzagalli et al., 2002; Sato et al., 2001; Streit et al., 2003; Holmes et al., 2003; Eimer et al., 2003; Eimer \& Holmes, 2002; Streit et al., 2000). As the amygdala is the key structure in the subcortical route (dashed lines in Figure 21) of evaluating emotional stimuli in order to convey information about potential threat (LeDoux, 1996; Morris et al., 2001), it is thought to be activated prior to other regions. Although the amygdala discriminates emotional from neutral faces already after approximately $100 \mathrm{~ms}$ (Streit et al., 2003; Liu et al., 1999), other areas respond to 
emotional information from faces at similar latencies indicating that the detection and crude affective categorization of facial expression is not purely mediated by an ultra-fast retinal-collicular-pulvinar route to the amygdala.

Unconscious emotional encoding is by definition automatic and the strongest evidence for unconscious emotional evaluation comes from studies on affective priming. Affective priming studies make use of the phenomenon in which the presentation of an emotionally laden stimulus influences participants' evaluative judgements of subsequently presented neutral stimuli. In the context of the consciousness question the most relevant studies are those demonstrating that the emotional meaning of a subliminally presented stimulus can influence subsequent judgements and the neuronal activity in the amygdala.

Numerous behavioural studies provided evidence that the emotional value of stimuli can be registered without the need for conscious awareness (Murphy \& Zajonc, 1993; Murphy et al., 1995; Rotteveel et al., 2001). A number of neuroimaging studies using backward stimulus masking (Esteves \& Öhman, 1993; Rolls \& Tovee, 1994) have demonstrated amygdala acitivity when subjects are stimulated with subliminal facial expressions (Killgore et al., 2004; Liddell et al., 2005; Morris et al., 1998, 1999; Nomura et al., 2004; Öhman, 2002, 2005; Öhman et al., 2007; Whalen et al., 1998). In contrast to these findings, an fMRI study by Pessoa and colleagues (2006) reported only differential responses in the amygdala to masked fearful faces relative to masked neutral ones when the participants were objectively aware of the faces. Thus, when the subjects were unaware of the fearful faces, no activity in the amygdala was observed indicating that visual awareness and stimulus visibility strongly affect amygdala responses.

Binocular rivalry is an alternative method to present visual stimuli subliminally. If two different stimuli are separately projected on corresponding retinal locations of the two eyes, rather than appearing as a mixture, they will compete to determine the percept. Given that the two stimuli are roughly matched in salience, the percept will spontaneously shift between them. However, if one stimulus is in some sense more salient than the other, it will suppress the other, which implies that the suppressed stimulus is presented outside of the subject's awareness. Using a binocular rivalry paradigm two studies have reported reliable amygdala activation to suppressed images of fearful faces (Pasley et al., 2004; Williams et al., 2004). These data strongly suggest a subcortical origin of the visual input that allowed the amygdala to discriminate suppressed fearful from suppressed neutral faces in the absence of cortical processing. Further evidence for a rapid subcortical route to the amygdala is provided by studies with patients suffering blindsight, due to striate cortex 
damage (de Gelder et al., 1999; Morris et al., 2001), and parietal patients with visual extinction and spatial neglect (Vuilleumier et al., 2002) in which an increase in amygdala activity was obtained during discrimination of emotional facial expressions without awareness.

Although a great number of studies support the notion that some aspects of emotional meaning can be encoded very quickly and without conscious awareness, these studies do not directly address whether such encoding is resource-independent. It is conceivable that even quick, unconscious encoding of emotion could demand some measure of attentional resources. Moreover, attention is directed to the location of the stimulus and generally there is no competing task using attentional resources. Several studies to date have examined whether emotional encoding demands a certain measure of attentional resources, however, the issue is still unresolved because the results presented a mixed picture.

One of the first fMRI studies dealing with this issue directly compared the influence of emotional expression and that of selective attention on face processing, by manipulating attention and emotion orthogonally, while keeping the task demands identical across all conditions (Vuilleumier et al., 2001). Participants were instructed to match the identity of two faces (neutral and fearful) or houses, each pair being aligned either vertically or horizontally at eccentric locations. While the participants attended for example to the faces, they had to ignore the house stimuli presented simultaneously at the other two locations. Responses in the amygdala to fearful faces were not significantly reduced when the faces were unattended leading to the conclusion that fear-responses mediated by the amygdala may be obligatory and not dependent upon focused attention (Figure 38). Furthermore, it could be demonstrated that spatial attention strongly modulated activity in extrastriate visual areas, with greater responses in bilateral fusiform cortex when faces were attended and in parahippocampal cortex when the houses appeared at the relevant locations.

The same results have been reported by Anderson et al. (2003) presenting faces (fearful, disgusted, neutral) superimposed on pictures of places in the fovea. Participants had either to direct their focus of attention to the faces and perform a male/female judgement or to the houses and make an inside/outside judgement. The amygdala response to fearful faces was not significantly reduced with reduced attention, supporting the concept of automatic processing. 
(A)

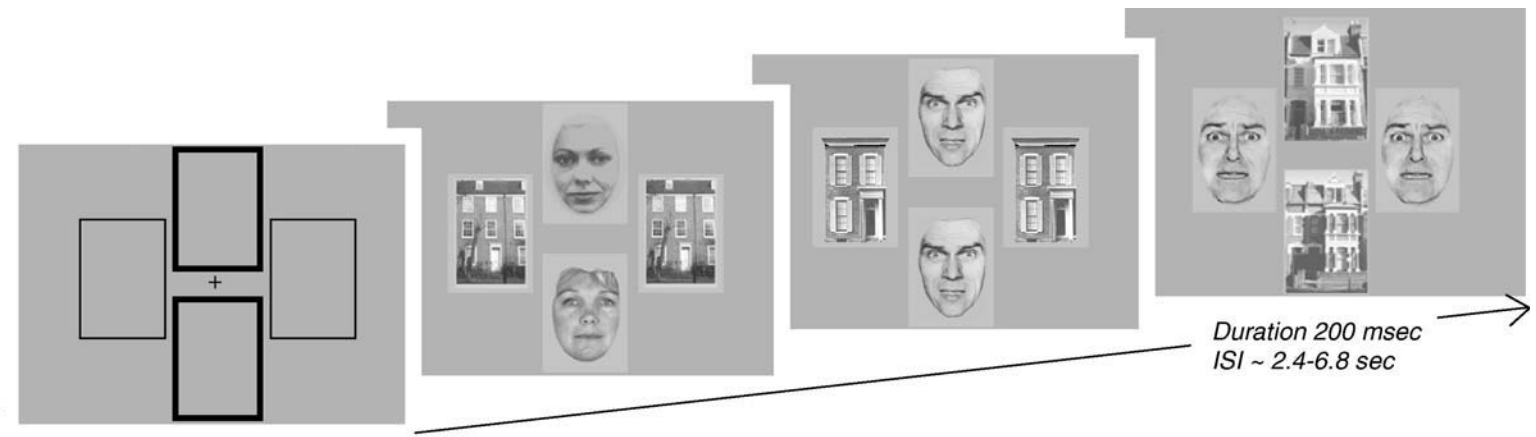

(B)
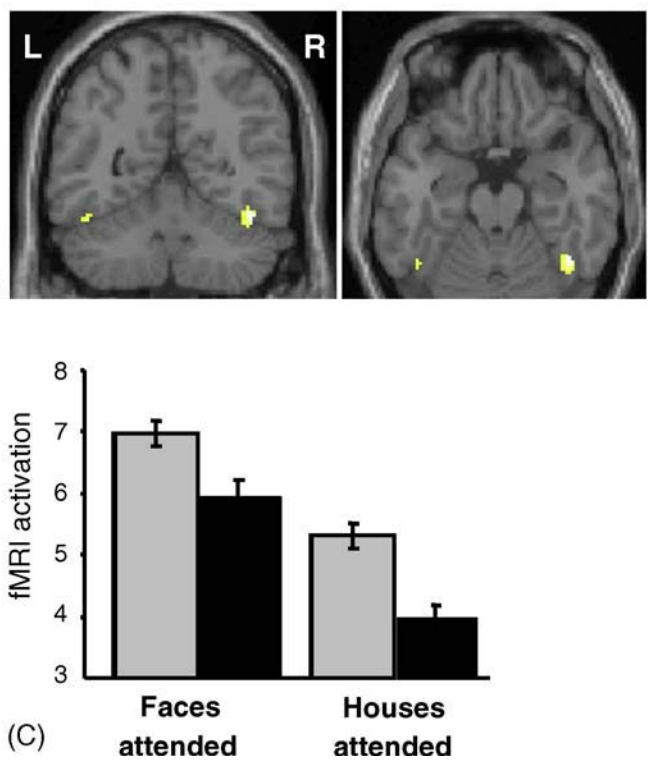

(D)
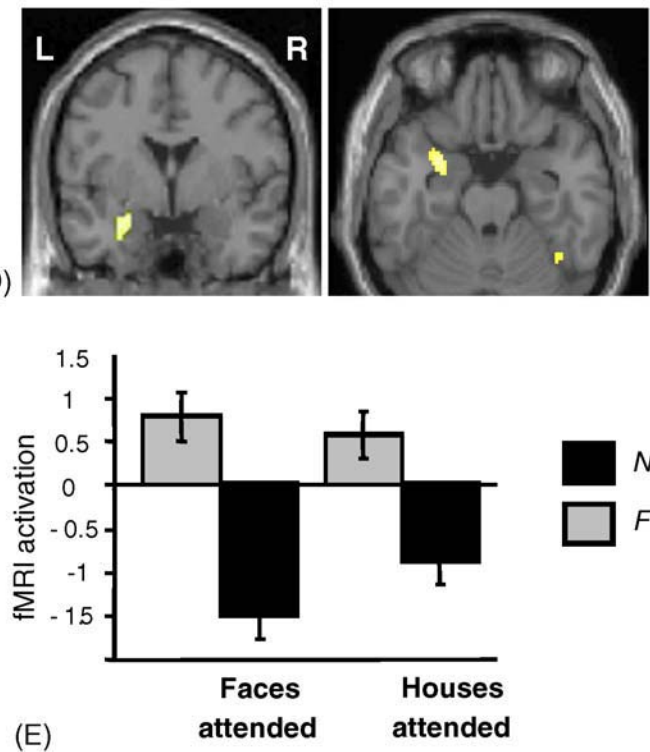

Neutral faces Fearful faces

\section{Figure 38}

Additive effects of attention and emotion on fMRI responses to faces. (A) On each trial, two houses and two faces (with either fearful or neutral expression) are briefly shown $(200 \mathrm{~ms})$, while subjects concentrate on two pre-specified locations (either horizontal or vertical) to judge whether the pictures appearing at these two locations are the same or different. (B) Face-specific areas in fusiform cortex are activated bilaterally when faces appear at the attended locations (as opposed to houses). (C) Activity in the right FFA show a marked decrease when faces appear at ignored locations, equally so for both fearful and neutral faces. However, FFA activity is increased when fearful (as opposed to neutral) faces are present in the display, and this effect is independent (i.e., additive) from the modulation by spatial attention (i.e., still present when faces appear at ignored locations). (D) Amygdala is also activated by fearful (versus neutral) faces regardless of whether faces appear inside or outside the focus of attention (E), indirectly suggesting that residual amygdala responses to ignored faces might drive the additive effects of emotion on fusiform cortex in this situation, concomitant to the modulations by selective attention (from Vuilleumier \& Pourtois, 2006).

Moreover, an fMRI study investigating the differential neuronal responses related to emotional stimuli with and without conscious perception in a patient with left neglect and extinction (lesion centred on the right posterior inferior parietal region) revealed that fearful faces activated the left amygdala and extrastriate visual cortex both when seen and extinguished (Vuilleumier et al., 2002). However, the comparison of perceived versus extinguished faces showed no difference in amygdala response to fearful faces, indicating 
no interaction between emotion and awareness in the amygdala. Therefore, these results suggest that emotional inputs to the amygdala may be at least partly independent of processing in occipital and fusiform cortex associated with visual awareness. It is proposed that early in stimulus processing the amygdala receives input about the emotional significance of a stimulus, and through projections to sensory cortical regions, modulates further attentional and perceptual processes (Anderson \& Phelps, 2001). In support of this model, an fMRI study conducted in patients with medial temporal lobe damage showed that the amygdala is mediating enhanced responses in visual cortical regions for emotional stimuli (Vuilleumier et al., 2004). Fearful and neutral faces were presented to normal control subjects, patients with damage limited to the hippocampus, and patients with damage to the hippocampus and amygdala. Enhanced activation was observed in visual cortical regions for fearful versus neutral faces in normal control subjects and in patients with damage confined to the hippocampus. However, patients with damage to the amygdala did not show any significant activation for fear versus neutral faces in the visual cortex indicating that the amygdala plays a critical role in mediating the transient changes in visual cortical processing that occur for emotional stimuli.

In summary, converging evidence from various methodologies is lending support for the idea that the emotional qualities of stimuli are processed automatically. Psychophysiological studies have consistently demonstrated that categorical perception of emotional expressions can be encoded relatively early within the processing stream, although the link between speed and automaticity is open to question. Furthermore, a number of studies suggest that attention and awareness have little impact on the amygdala's response to fearful stimuli. It has been proposed that an early, automatic amygdala response to fear and threat stimuli is an important factor in its ability to modulate attention and responses to potential danger (Davis \& Whalen, 2001). However, such findings do not necessarily imply that these emotional effects would be entirely immune to any task-related attentional influences, since increasing perceptual load (Lavie, 1995) or competition between stimuli (Kastner \& Ungerleider, 2000) might conceivably reduce the degree of amygdala responses to emotional stimuli. 


\subsubsection{Controlled processing}

In contrast to neuroimaging studies supporting the view that the determination and identification of emotional facial expressions are independent of visual awareness, stimulus visibility and attention, a growing number of studies indicates that processing emotional stimuli requires the availability of attentional resources. This position adopts the biased competition model of attention (Desimone \& Duncan, 1995) and the attentional load theory (Lavie, 1995). As mentioned in the previous chapter about attention, according to the biased competition model attention is the product of the competition for neuronal representation which is biased by both bottom-up sensory-driven mechanisms and topdown influences generated outside the visual cortex. Faces are not considered as a 'privileged' category of objects immune to the effects of attention. Instead facial expressions must compete for neuronal representation, just as neutral stimuli do, which is illustrated within the context of the biased competition model of attention in Figure 39.

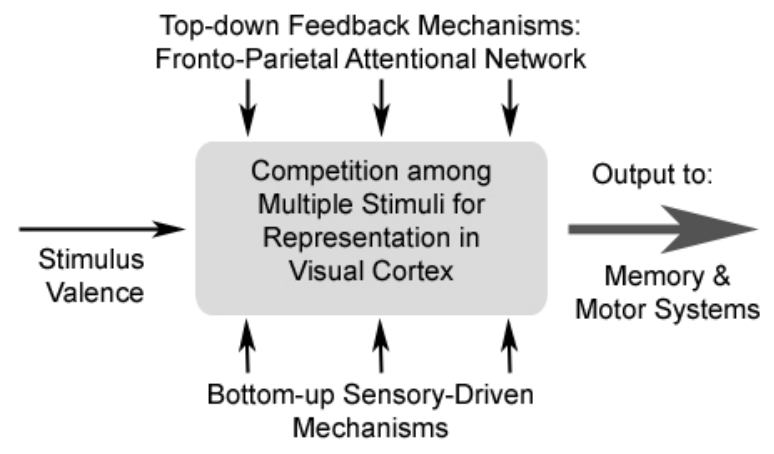

\section{Figure 39}

Biased competition model of visual attention and the processing of emotion-laden stimuli. Facial expressions must compete for neuronal representation (see arrow labeled 'stimulus valence') like neutral stimuli (from Pessoa et al., 2002).

Perceptual load determines the extent to which irrelevant distracters are processed and the degree to which representational features of a visual stimulus require augmentation by topdown influences for successful performance (Figure 40). According to this model, it has been predicted that an increase of perceptual load leads to an increase of priming of the representational features of the task-relevant stimulus and because of stimulus competition, will result in greater suppression of competing representations. In other words, high perceptual load that engages full capacity in relevant processing would leave no spare 
capacity for perception of task-irrelevant stimuli. On the other side, in situations of low perceptual load any capacity not taken up in perception of task-relevant stimuli would involuntarily 'spill over' to the perception of task-irrelevant distracters. Increased perceptual load means that either the number of different-identity items that need to be perceived is increased, or that for the same number of items perceptual identification is more demanding on attention.

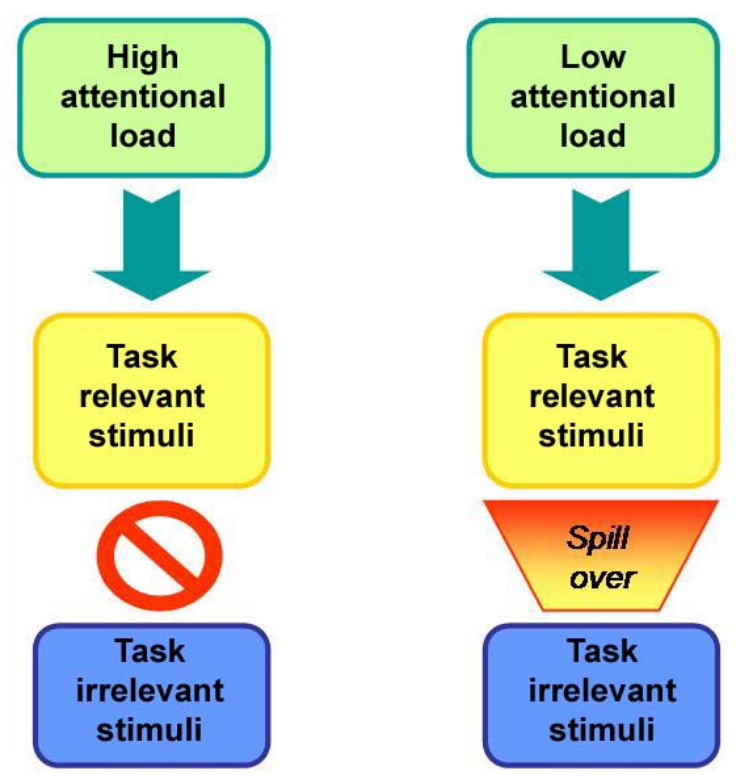

\section{Figure 40}

Attentional load model. Under conditions of high-attentional load only task-relevant stimuli can be processed because of the limited processing capacity of the visual system. In contrast, if the processing load of a target task does not exhaust available capacity, remaining resources spill over to process task-irrelevant stimuli.

Evidence for this load theory has been obtained in a number of studies using different load manipulations varying either the number of stimuli to be processed (e.g., relevant search set size) or the processing requirements for those stimuli (e.g., comparing tasks of easy detection versus hard discrimination) (Lavie, 1995; Lavie \& Cox, 1997; Lavie \& Fox, 2000; Rees et al., 1997; Lavie, 2005; Erthal et al. 2005; Yucel et al., 2007). In line with this view, Pessoa and colleagues $(2002,2005 \mathrm{a}, \mathrm{b})$ have argued that emotional facial expressions are not a 'privileged' category of stimuli immune to the effects of attention, but rather, a class of stimuli that must compete for neuronal representation. Furthermore, it has been criticized that the implemented task by Vuilleumier and colleagues (2001) (matching the identity of houses) was not sufficiently attentionally demanding and might 
have allowed attentional resources to 'spill over' and process the facial expressions (Pessoa et al., 2002).

Pessoa and colleagues (2002) used fMRI to investigate the influence of attentional load on activity in brain regions that are responsive to emotional faces (neutral, happy and fearful faces). Next to the foveally presented expressive faces, a more demanding secondary task (judging the orientation of two similarly oriented lines in the periphery) was used to take away attentional resources. The results showed that when participants had to deploy their attention to the bar orientation task, the amygdala responsiveness to the emotional faces was reduced compared to when participants attended to the faces (Figure 41). The conclusion was drawn that emotion encoding is not immune to competition from other operations and requires some degree of attention.
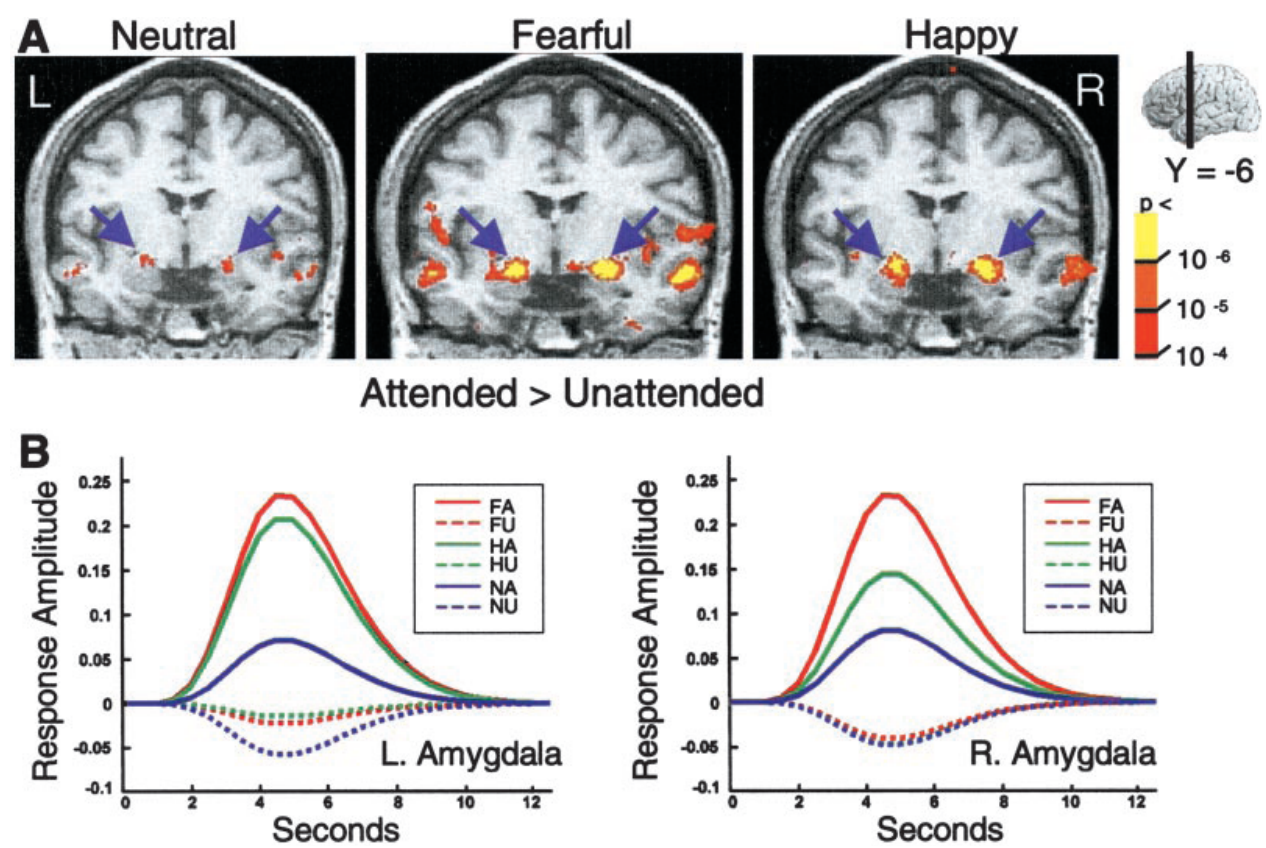

\section{Figure 41}

Attention and valence effects in the amygdala. (A) Arrows point to the amygdala. Attended faces compared with unattended faces evoked significantly greater activations for all facial expressions. The level of the coronal section is indicated on the small whole-brain inset. L, left; R, right. (B) Estimated responses for the left and right amygdala ROIs as a function of attention and valence. FA, fearful attended; FU, fearful unattended; HA, happy attended; HU, happy unattended; NA, neutral attended; NU, neutral unattended (from Pessoa et al., 2002).

To further directly test the impact of attentional load on the processing of emotional faces, Pessoa and colleagues (2005b) used the same task as in their previous study (Pessoa et al., 2002), but created three levels of attentional load (low, medium, and high) by varying the angular difference of the bars. The results revealed differential amygdala activation for 
unattended neutral and fearful faces at fixation only during the low-load condition, and an absence of emotion-related responses in the medium-load and high-load conditions. Furthermore, signal decreases (relative to fixation) in a network of brain regions including the amygdala have been reported during the high-load bar-orientation task alone. These findings demonstrated that amygdala responses are modulated by an attentional manipulation even when the task is fixed and only difficulty changes. In line with the results from the studies by the group of Pessoa, two other studies reported differential amygdala activation to faces using a letter search task of high and low perceptual attentional load superimposed on fearful or neutral faces presented foveally. In one study amygdala activation was only observed during the low-load task (Bishop et al., 2007) and in the other one the amygdala activation was eliminated for both levels of load (Mitchell et al., 2007).

However, several issues make it difficult to interpret these neuroimaging data. First it has to be noted that because of the relative low temporal resolution of the fMRI method, it would be be possible that the initial encoding of emotional information may be relatively resource-free but that top-down, controlled processes become involved as more time passes. fMRI methodology is unable to separate relatively fast versus slow encoding processes due to the slow time course of the haemodynamic response (Aguirre et al., 1998; Miezin et al., 2000). Although the reviewed studies suggested that emotion-related processing is influenced by attentional load, they did not isolate the earliest processing stages, which are most likely to occur in an automatic rather than in a controlled fashion. Unfortunately, ERP studies have not helped to clarify this issue. ERPs are particularly suited for studying the time course of emotional processes and investigating whether and when the processing of emotional stimuli is modulated by selective attention. Using the same experimental paradigm as Vuilleumier and colleagues (2001), an ERP study reported an enhanced positivity elicited by attended fearful faces relative to neutral ones, with an early frontal effect (between 100 and $120 \mathrm{~ms}$ ) followed by a more broadly distributed emotional positivity (Holmes et al. 2003). However, these emotional expression effects were completely eliminated on trials where faces were presented at unattended locations. These findings could be even extended by demonstrating no emotional expression effects for all six basic emotions when attention was directed away from the faces toward a demanding perceptual discrimination task (Eimer et al., 2003). Therefore, brain processes involved in the detection and analysis of emotional facial expression do not occur preattentively and are gated by spatial attention. Another two studies found ERP evidence 
consistent with the notion that the differential brain responses to unattended emotional stimuli depend strongly on the attentional load of the ongoing task (Doallo et al., 2006; 2007). All these ERP studies presented the emotional stimuli peripherally, while the attentional load was modulated at fixation. Though, a recent ERP experiment found that the extent to which the emotional processing is influenced by attention depends on factors such as foveal versus peripheral presentation (Holmes et al., 2006). When faces were presented foveally, the initial rapid stage of emotional expression processing was unaffected by attention which is in direct contrast to the other ERP studies.

Furthermore, in the Pessoa studies (Pessoa et al., 2002; 2005b), a task-related confound might limit the interpretation of the results. Specifically, on trials when participants had to attend to the faces, they had to make judgements about the gender of the face whereas on ignore-faces trials, they had to perform the bar-orientation task. Thus, not only the focus of attention but also the decision type varied among conditions. In contrast, the Vuilleumier study (Vuilleumier et al., 2001) employed the same task (match/mismatch judgement) for both attended-faces and ignore-faces trials, providing a cleaner comparison.

Moreover, Vuilleumier points out (Vuilleumier \& Pourtois, 2006) that in the study by Pessoa (2002) a modulation of activity in the amygdala and fusiform gyrus occurred equally for all categories of faces, regardless of their emotional valence, and no response above baseline was found in the fusiform cortex when faces were ignored even though these were presented at fixation. Since a blocked design was used and faces were presented on each trial in the attended condition, it remains unclear, whether the main changes in activity found in this study reflected stimulus-driven responses to emotional faces, or rather increased baseline activity through strategic top-down influences on the amygdala as well as on the fusiform gyrus.

\subsubsection{Neuromodulatory factors}

The processing of emotion-laden information in the brain, especially the activity in the amygdala, depends on a wide array of factors that ultimately determine the strength of evoked responses. Some of the most important factors related to this issue will be discussed in the following sections, namely the interaction between attentional load and spatial positioning of the affective stimuli, as well as the spatial frequency of the emotional stimuli and the effects of stimulus salience. 


\subsubsection{Interaction between attentional load and location of affective stimuli}

One explanation for the discrepant imaging results has been provided by Palermo and Rhodes (2007), by considering not only the attentional load, but also the spatial positioning of the to-be-ignored stimuli in the visual field. Indeed, the pattern of results to date suggests that peripherally presented unattended fearful faces may activate the amygdala under both low (Vuilleumier et al., 2001) and high (Williams et al., 2005) attentional load manipulations. On the contrary, ignored fearful faces presented at fixation may lead to greater amygdala activation under low-load (Anderson et al., 2003; Pessoa et al., 2005b) but not high-load (Pessoa et al., 2002; 2005b) conditions (Figure 42). Therefore, studies varying both attentional load and location of the emotional stimuli are needed to test the interaction hypothesis outlined above.
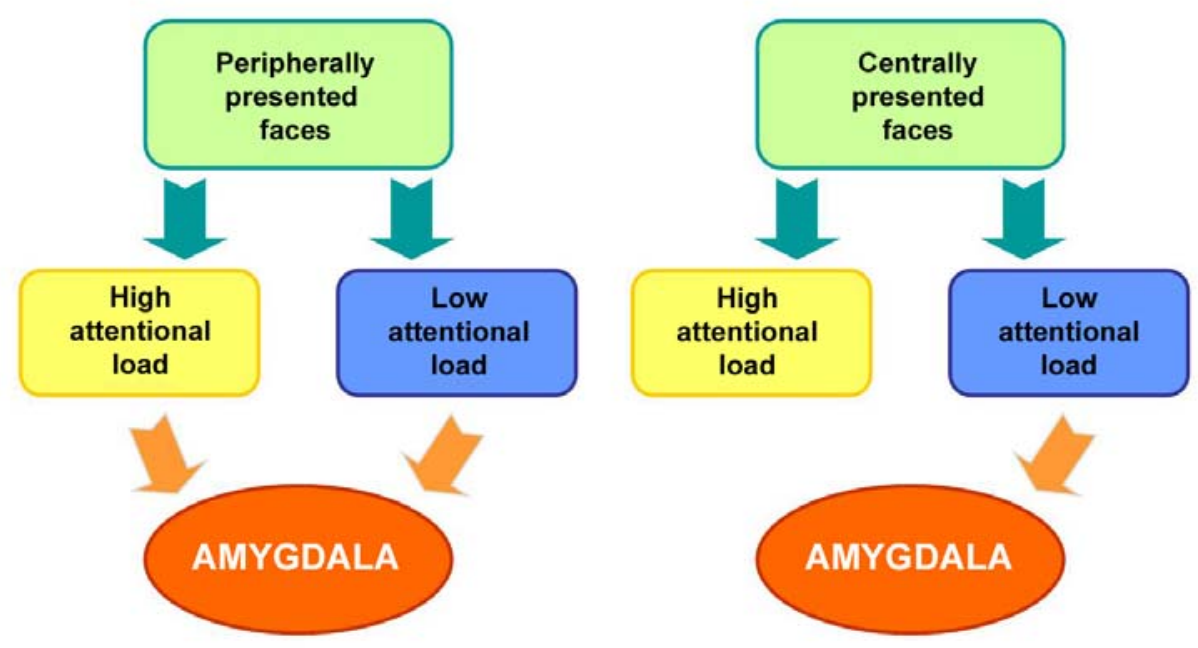

\section{Figure 42}

Interaction between attentional load and location of emotion-laden stimuli.

To date only one recent fMRI study (Silvert et al., 2007) investigated whether the processing of peripheral face stimuli can be influenced by attention under high perceptual load conditions. Pairs of houses and faces (fearful and neutral) were presented simultaneously in the periphery at the corners of an imaginary square centred on a fixation cross. The stimuli were tilted slightly either clockwise or anticlockwise. Participants had to perform two attentional tasks of varying difficulty, they had to match two of the pictures along one diagonal either for orientation (low-load) or for identity (high-load). The right amygdala was activated more strongly by fearful faces than neutral ones and when subjects 
attended to the faces rather than houses. During the high-load identity task the differential amygdala response to emotional versus neutral unattended faces vanished, whereas in the low-load orientation task a significant interaction between emotion and task was obtained. These findings are consistent with the proposal that amygdala processing depends on the attentional resources available. However, it is important to note that the amygdala response was not completely suppressed in relation to unattended fearful and neutral faces during the high-load task, indicating that not all attentional resources have been taken away from the competing task. Therefore, intermediate levels of inattention would render the emotional expression of unattended faces increasingly ambiguous, which could lead to undifferentiated amygdala activation. It has been proposed that if attention is more efficiently diverted from the faces, the amygdala responses would gradually lose their specifity.

\subsubsection{Spatial frequency of affective stimuli}

The proposal about the interaction between attentional load and the location of the emotional stimuli leads to the assumption that the amygdala may be especially sensitive to affective stimuli presented in the periphery, because one central role of this structure is to direct the attentional focus to emotionally relevant stimuli in the visual field. As mentioned earlier it has been proposed that the amygdala may receive direct subcortical inputs from the thalamus to enable crude but fast signals about threat-related stimuli to bypass slower cortical analysis in the ventral visual pathways (LeDoux, 1996). Specifically, a retinalcollicular-pulvinar pathway has been suggested (Morris et al., 1999; de Gelder et al., 1999). Therefore, a rapid reaction to behaviourally relevant stimuli might be associated with a cost of some precision. This capability is favoured by the existence of two different neuronal systems for processing visual information: the magnocellular $(\mathrm{M})$ and the parvocellular (P) pathway (Livingstone \& Hubel, 1987; 1988; Kandel et al., 2000; Bullier, 2001).

M-cells are particularly sensitive to rapid temporal change such as luminance flicker and motion, and have large receptive fields making them sensitive to peripheral stimuli. Furthermore, M-cells contribute most to vision requiring low spatial and high temporal resolution and produce rapid, transient, but coarse visual signals. Thus, these cells have a potential advantage in the perception of sudden appearance, location, direction of movement, and stimuli signalling potential danger. On the contrary, the P-cells are 
responsive to stimuli of high spatial but low temporal frequency. The neurons of the Ppathway are highly sensitive to wavelength and orientation, and have small receptive fields that show enhanced sensitivity to foveal information. Therefore, P-cells are crucial for sustained, analytic, and detailed processing of shape and colour. Both pathways project to distinct cortical regions, with the M-pathway projecting mostly to dorsal visual areas, including V2 and V5/MT (Shipp, 2001), and connected subcortical regions, such as the superior colliculus and pulvinar (Schiller et al., 1979; Leventhal et al., 1985; Berson, 1988; Berson \& Stein, 1995), whereas the P-pathway provides input to ventral visual cortex allowing a deep exploration of stimulation (Merigan \& Maunsell, 1993). Faces provide complex visual information at multiple spatial frequencies, potentially ranging from global configurational properties sufficient to provide only coarse emotional cues revealed by low-spatial frequency components (Costen et al., 1996; Schyns \& Oliva, 1999; Calder et al., 2000) to fine grained features important for precise recognition of identity and for more detailed analysis of facial traits, such as age or expression-related wrinkles conveyed by high-spatial frequency components (Liu et al., 2000; Hayes et al., 1986; Fiorentini et al., 1983; Norman \& Ehrlich, 1987).

Recently, fMRI studies tested whether face processing in ventral visual cortex and emotion processing in the amygdala are differentially sensitive to high versus low spatial frequency inputs (Vuilleumier et al., 2003; Winston et al., 2003). Therefore, the spatial frequency of pictures of fearful and neutral faces was filtered to produce facial stimuli that retained only high or low frequency spatial information under the assumption that the low road via the superior colliculus and pulvinar operated primarily on gross, low-frequency information. Indeed, it could be demonstrated that high spatial-frequency information in faces travels via parvocellular inputs into ventral cortical pathways and evokes responses in fusiform cortex, whereas the amygdala preferentially responds to low spatial frequency information carried primarily by magnocellular visual pathways. Additionally to the amygdala, increased activity was also observed in the superior colliculus and the pulvinar for fearful expressions presented with low spatial frequency suggesting that these subcortical pathways may provide coarse, but rapid, fear-related inputs to the amygdala, independent of slower conscious analysis based on high spatial frequency in cortical pathways. It can be concluded that these distinct response properties delineate segregated anatomical routes for facial and emotional processing. Furthermore, these results indicate that amygdala may be especially sensitive to peripherally faces. 
Another fMRI study (Rotshtein et al., 2007) revealed dissociable processing in occipitotemporal cortex, with distinct regions specialized in processing low and high spatial frequency components from faces. Specifically, high spatial frequency faces evoked increased activation in the right inferior occipital gyrus and left inferior temporal gyrus, whereas bilateral middle occipital gyrus responded to low spatial frequency information. A common effect of high and low spatial frequency was observed in the right fusiform gyrus. A connectivity analysis suggested direct influence of the middle occipital gyrus, inferior temporal gyrus and inferior occipital gyrus on the fusiform gyrus responses. On the basis of these findings it can be assumed that different regions within occipito-temporal cortex extract distinct visual features at different spatial frequencies in faces and that this information is passed on to the right fusiform gyrus, where these different visual cues may converge.

\subsubsection{Stimulus salience}

Stimulus salience is only one of a number of stimulus properties that attract attention in a bottom-up fashion. Under controlled conditions, the conspicuousness of a stimulus, also referred to as its salience, depends strongly on local feature contrast. The salience of a stimulus is related to the contrast between its features and the features of its neighbours. Functionally speaking, salience is determined by the visual uniqueness of a stimulus within the context of a scene. Numerous studies have shown that the presentation of a salient distracter in a display increases the search time for a target relative to the presence of a nonsalient distracter (Eltiti et al., 2005; Mounts, 2005; Theeuwes, 2005; Yantis \& Jonides, 1990). This increase of the search time implies that salience biases the processing in favour of the distracter. However, distracter processing is also determined via top-down attentional processes depending on the perceptual load of the task. Therefore, it is of great importance to consider the effects of stimulus salience on the processing of emotional unattended faces.

To date only one fMRI study tested whether bottom-up and top-down factors have differential effects in shaping responses evoked by unattended emotional faces (Hsu \& Pessoa, 2007). Additionally to a circular array of letters (main task) displayed in the centre of the visual field, a pair of peripherally presented faces (neutral and fearful), positioned to the left and right of fixation, served as distracters. To test the impact of top-down mechanisms on the processing of task-irrelevant faces, the attentional load was 
manipulated in the main task by presenting a target letter among several distinct nontarget letters (attentional load condition) or among an array comprised of the same nontarget letter (baseline condition). To investigate the effect of bottom-up factors, stimulus salience was manipulated by reducing the contrast and the size of the letters relative to that in the baseline condition (salience condition). The results are in line with previous studies in demonstrating that the perception of both fearful and neutral faces was modulated by topdown attentional processes. When the attentional load of the main task was increased relative to baseline, responses evoked by fearful and neutral face distracters decreased in the amygdala and the fusiform gyrus. By contrast, decreasing the target salience resulted in increased responses relative to baseline in the amygdala and fusiform cortex. Another important finding was that time-related effects were observed depending on the task. During the salience condition, amygdala and fusiform gyrus responses to distracters decreased over the experimental time course whereas during the attentional load condition, responses increased over trials. Taken together, these results indicate dissociable effects of bottom-up and top-down factors on the processing of unattended fearful faces. 


\section{Face processing in the human amygdala depending on attentional load and spatial}

location

\section{$\underline{6.1 \text { Introduction }}$}

The amygdala has been identified to play a crucial role in the processing of emotional stimuli (Sergerie et al., 2008; Zald, 2003; Phan et al., 2002). It provides a coarse, rapid and automatic perception of emotional stimuli based on a subcortical route via the superior colliculus and the pulvinar nucleus of the thalamus, whereas a fine-grained perceptual representation of emotion-laden stimuli involves the occipital and temporal visual neocortex (Adolphs, 2002a; LeDoux et al., 1984; LeDoux, 1996). Through reciprocal connections to sensory cortical processing regions, such as the visual cortex (Amaral et al., 2003), the amygdala may mediate the facilitation of attentional and perceptual processes (Anderson \& Phelps, 2001; Phelps \& LeDoux, 2005; Phelps, 2006; Morris et al, 1998a; Vuilleumier et al., 2004). Although a great number of studies support the notion that some aspects of emotional meaning can be encoded very rapidly and without conscious awareness (Morris et al., 1998b, 1999; Whalen et al., 1998; Liddell et al., 2005; Öhman et al., 2007; Killgore et al., 2004; Öhman, 2002, 2005), these studies do not directly address whether such processing demands attentional resources. Recent neuroimaging studies presented conflicting evidence bearing on this issue.

The processing of emotion-laden stimuli in the brain, especially the activity in the amygdala, depends on a wide array of factors that ultimately determine the strength of evoked responses. The previous findings condense into the view that the attentional load as well as the spatial position of the emotional stimuli have a great impact on the neuronal responses of the amygdala to faces (Vuilleumier et al., 2001; Williams et al., 2005; Anderson et al., 2003; Pessoa et al., 2002, 2005b; Silvert et al., 2007). However, no fMRI study to date varied both factors to directly test the interaction between them. Furthermore, only few studies manipulated attentional load in the presence of faces presented in the periphery and therefore presumably minimized the role of the magnocellular pathway in conveying information concerning facial expression to the amygdala. 
In the present study I investigated the effects of variable attentional load and stimulus location on the processing of emotional faces in the amygdala using fMRI. Pairs of faces were presented horizontally at three distinct spatial positions in the visual field: Adjacent to the central fixation point, on the edge of the possible portrayed visual field on the screen and in between these two extreme positions. In addition to the face stimuli, four streams consisting of letters and digits displayed in RSVP were presented simultaneously in each visual field quadrant (Morawetz et al., 2007). During the attend-faces conditions, participants had to direct their attentional focus to the faces and match either the gender of the face stimuli or the valence of the facial expression (neutral, happy and fearful). During the ignore-faces conditions, subjects had to deploy their attention to either one (low-load) or two RSVP streams (high-load) along one diagonal performing a match/mismatch judgement. This experimental design allowed me to manipulate the attentional load, while the stimulus display did not change across runs. Thus, the decision type (match/mismatch judgement) was identical for attend-faces and ignore-faces conditions, thereby facilitating a clean comparison between them. Furthermore, I was able to directly test the interaction between attentional load and stimulus location in the visual field within the same group of participants.

According to the view that faces are processed preattentively, I supposed that the amygdala would be activated regardless of task-relevancy of the emotional stimuli. In contrast, an increased activation of the amygdala during attend-faces conditions and a reduced or eliminated response during ignore-faces conditions would support the concept that the processing of emotional stimuli requires the availability of attentional resources. In regard to the spatial location of the emotional stimuli, I expected an enhanced amygdala response to centrally presented faces during the low, but not high attentional load condition. On the contrary, I assumed that activity in the amygdala would increase during both high and low attentional load conditions when the faces are presented more peripherally.

\subsection{Materials and Methods}

\subsubsection{Subjects}

Fourteen right-handed, healthy adults (9 males) aged 20-34 years (mean \pm SD: $25.8 \pm 3.0$ years) with normal or corrected to normal vision were examined. All subjects gave written informed consent to participate in the study, which was approved by the local ethics committee. 
Prior to the imaging sessions, participants completed the Positive and Negative Affective Schedule (PANAS; Watson, Clark and Tellegen, 1988) to assess different current emotional states and feelings as well as a self-report measure of attentional control, the Attentional Control Scale (ACS; Derryberry \& Reed, 2002).

The PANAS includes 20 items that assess two orthogonal factors of affective state, positive affect and negative affect (Watson \& Tellegen, 1985). Scores on each scale range from 10 to 50. On the PANAS, participants' positive affect scores ranged from 22 to 35 (mean \pm SD: $28.2 \pm 3.2)$ and their negative affect scores from 10 to $17(12.8 \pm 2.5)$. These scores are similar to the published norms (positive affect: $29.7 \pm 7.9$; negative affect: 14.8 \pm 5.4; Watson, Clark and Tellegen, 1988).

The ACS consists of 20 items to assess individual differences in attentional skills related to voluntary executive functions. The scale measures a general capacity for attentional control, with correlated sub factors related to the ability to focus attention, to shift attention between tasks and to flexibly control thought (Derryberry \& Reed, 2002). Scores on the ACS range from 20 to 80 . Participants' scores ranged from 47 to $66(55.6 \pm 6.2)$ comparable to the results of Bishop et al. (2007).

Furthermore, subjects rated the valence and the level of arousal of all face stimuli using a computerized version of the Self Assessment Manikin (SAM) scale (Bradley \& Lang, 1994). The SAM is a self-administered scale with icons that indicate graphically how arousing and pleasant stimuli are. Participants rated each face picture on pleasantness (ranging from extremely pleasant to extremely unpleasant) and arousal (ranging from extremely aroused to extremely calm). The version of the SAM used in the present study included five icons that define a 9-point scale for each dimension with 9 representing a very pleasant and extremely exciting stimulus. The mean valence ratings for neutral $(4.2 \pm$ $0.4)$, happy (6.6 \pm 0.8$)$, and fearful $(2.9 \pm 0.6)$ stimuli showed that all faces were evaluated in accordance with the intended portrayed facial expression. The mean arousal ratings for fearful and happy faces indicated that these faces were neither perceived as very calm nor as extremely exciting (happy faces: $5.5 \pm 0.9$; fearful faces: $5.9 \pm 0.9$ ), whereas neutral faces were rated as less arousing $(3.7 \pm 0.6)$. 


\subsubsection{Stimuli}

The emotional stimuli consisted of greyscale face photographs of 70 individuals (35 male, 35 female), each portraying a happy, fearful and neutral expression and were selected from the Karolinska Directed Emotional Faces (Lundqvist, Flykt, and Öhman, 1998). All faces were presented within the same oval frame to avoid discrimination based on outer contours. As performance declines towards the visual field periphery in most tasks, when a constant stimulus size is used (Weymouth, 1958), we intended to equate task performance by increasing the size of the face stimuli towards periphery (Mäkelä et al., 2001). Therefore, the photographs were scaled by the human cortical magnification factor to activate an approximately equivalent portion of early visual cortex at all stimulated eccentricities (Van Essen et al., 1984). Pairs of faces were presented along the horizontal meridian for $550 \mathrm{~ms}$ and then masked by a Fourier-transformed image for $1050 \mathrm{~ms}$. Face stimuli located $1.68^{\circ}$ from central fixation (central position) had a height of $3^{\circ}$, pictures presented at a distance of $5.6^{\circ}$ from fixation (middle position) were $5^{\circ}$ high and photographs depicted at $11.25^{\circ}$ eccentricity (peripheral position) featured a height of $7^{\circ}$. The spatial positioning of the emotional faces remained the same during the stimulation blocks, but was varied in between experimental runs.

The letters and digits were displayed in RSVP. Four RSVP streams were presented simultaneously in each visual field quadrant. Each stimulus was shown for $183 \mathrm{~ms}$, which precluded deliberate saccades during their presentation and shifts of attention between processed target locations (Reeves \& Sperling, 1986; Weichselgartner \& Sperling, 1987; Peterson \& Juola, 2000; Duncan, 1994). The RSVP streams were placed $8.5^{\circ}$ diagonally from central fixation. The letter height of the stimuli subtended a visual angle of $2.3^{\circ}$.

All visual stimuli were presented on LCD-goggles (Resonance Technology, Northridge, USA) using the stimulation software Presentation (Version 10.3, Neurobehavioral Systems, USA). 


\subsubsection{Experimental procedure}

The experiment consisted of four conditions: Attend1, Attend2, Match gender and Match emotion (Figure 43).

In ignore-faces trials (Attend1 and Attend2), participants had to attend to the RSVP streams and perform two different tasks of low and high perceptual load. In the Attend1 task (lowload condition, Figure 43a) subjects had to maintain fixation on the fixation cross, and monitor one peripheral stream (lower left) of digits and letters for a predefined target which appeared every 1800-2000ms. The unattended RSVP streams served as distractors. Different digits were defined as targets, which were constant within a run but varied between runs. In the Attend 2 task (high-load condition, Figure 43b) subjects had to monitor two peripheral streams in opposing visual field quadrants for the simultaneous appearance of digits and indicate via button press whether the digits were the same (match) or different (mismatch).

In attend-faces trials (Match gender and Match emotion), subjects were required to perform an indirect or direct emotional processing task. In the indirect face task (Match gender, Figure 43c), subjects were instructed to maintain fixation on the fixation cross in the middle of the screen and indicate via button press whether the presented faces matched in gender or not. The direct emotional processing task (Match emotion, Figure 43d) required participants to identify the facial expression as fearful or happy, and press a button to indicate whether both faces showed the same expression or different ones. Neutral faces only served as distractors. All blocks of faces contained an equal number of males and females and an equal number of positive, negative and neutral expressions.

To summarize, the stimulus display consisted of a fixation cross in the middle of the screen, a pair of faces/Fourier-transformed images at one of the three spatial locations (central, middle, peripheral) and the four RSVP streams (Figure 43e).

The four experimental conditions were presented in a blocked fashion and were separated from each other by a fixation condition (20s) containing an instruction display that indicated the type of task (4s). During the fixation condition a black fixation cross was presented in the centre of a white screen. Experimental runs started with a fixation phase of $20 \mathrm{~s}$ and then altered between task (20s) and fixation condition (20s) resulting in a stimulation block of $160 \mathrm{~s}$, which was repeated three times (Figure 43f). Accordingly, one experimental run lasted $8 \mathrm{~min} 20$ s and was repeated four times during one scan session. Tasks and blocks were presented pseudo-randomized. Three scan sessions per subject were 
required to obtain the datasets for the three different spatial positions of the face stimuli in the visual field. Scan sessions were separated from each other by one week.

Participants had to respond as quickly and accurately as possible, by pressing one of two buttons on a fiber optic computer response device (Current Designs, Inc., Philadelphia PA, USA). Furthermore, subjects were explicitly told that fixation of the central cross should be maintained throughout the experiment. Only the focus of attention alternated between face stimuli and RSVP streams. Before scanning subjects were trained on the different tasks in separate sessions. In all blocks target match trials occurred with $50 \%$ probability. The adjusted frequency of correct answers ([\%correct/(\%correct - \%incorrect) $\left.]^{*} 100\right)$ was calculated for all four tasks for each participant in order to exclude subjects for whom the adjusted frequency of correct answers was not different from chance level. The behavioural data were analysed using a repeated-measures ANOVA. 
A

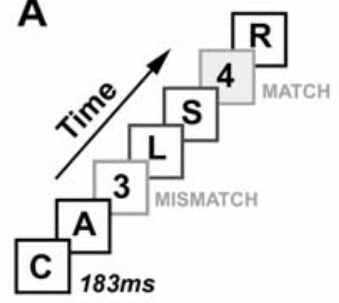

ATTEND 1

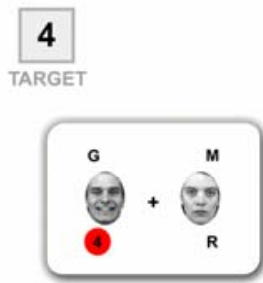

C

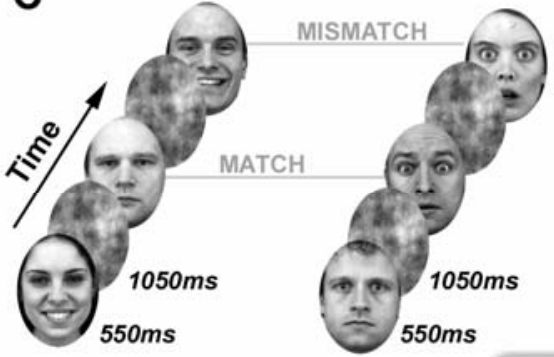

B

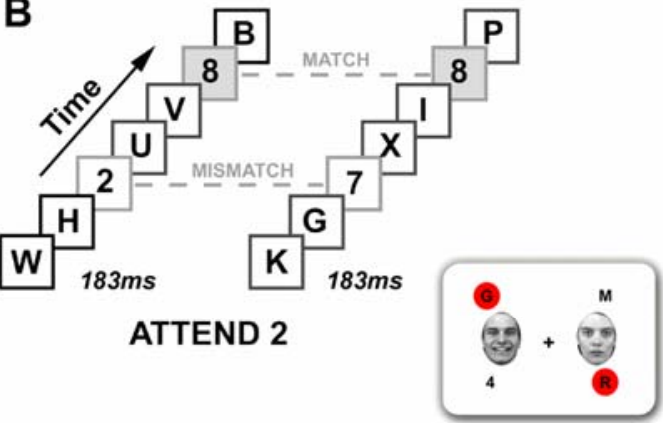

D

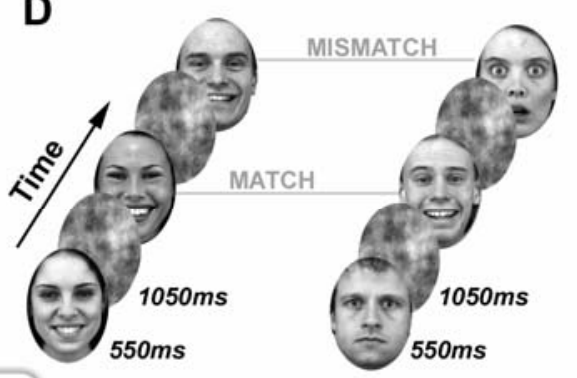

MATCH EMOTION

E

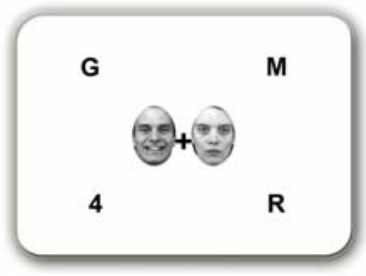

Central

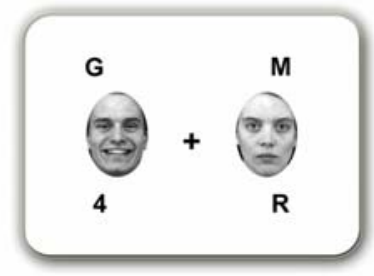

Middle

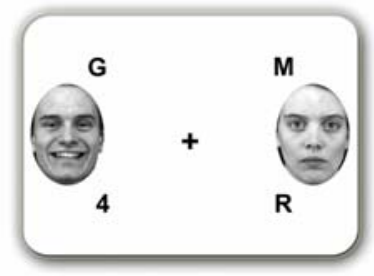

Peripheral

\section{F}
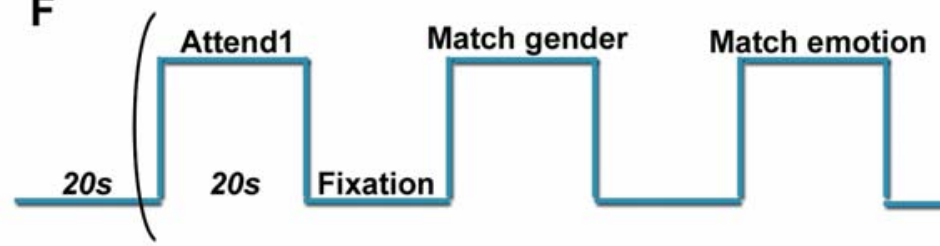

\section{Figure 43}

Tasks and experimental design. The visual display consisted of four rapid serial visual presentation (RSVP) streams presented simultaneously in each visual field quadrant and a pair of faces presented horizontally. (A) Attend1 task. Subjects had to monitor the lower left stream for the appearance of digits and indicate via button press if the digits matched or mismatched the target. Distractor streams had to be ignored. (B) Attend2 task. The upper left and lower right RSVP streams were defined as target locations. Simultaneously appearing digits were considered as targets. Participants had to indicate via button press whether the digits were the same (match) or different (mismatch). (C) Match gender task. Subjects had to attend the faces and determine whether they match in gender or not. (D) Match emotion task. Subjects had to attend the faces and match either fearful or happy facial expressions. Task relevance is indicated in red. (E) Spatial location of the face stimuli. Pairs of faces were presented either in the central, middle or peripheral location. (F) The stimulation paradigm employed for functional imaging. One block consisted of four different tasks, each lasting 20s, separated by a fixation condition and repeated three times. 


\subsubsection{Image processing and analysis}

For each experimental run 250 whole brain volumes were recorded. Data were analyzed within the framework of a random effects general linear model using BrainVoyager QX 1.9 (Brain Innovation, Maastricht, The Netherlands). Preprocessing of fMRI data included 3D-motion-correction, temporal high pass filtering (3 cycles/run), linear trend removal, spatial smoothing (Gaussian smoothing kernel, $4 \mathrm{~mm}$ full width half maximum), and transformation into the space of Talairach and Tournoux (1988).

In a first step, whole brain analysis was performed in order to identify regions that show a main effect of ignore-faces tasks (Attend1 and Attend2 versus Fixation) and a main effect of attend-faces tasks (Match emotion and Match gender versus Fixation) for each spatial position of the faces separately. The statistical threshold was set at a false discovery rate of $\mathrm{q}($ FDR $)<0.001$. In a second step, I tested for task-related differences by contrasting the different experimental conditions with each other (Match emotion versus Match gender; Attend1 versus Attend2; Match emotion versus Attend1; Match gender versus Attend1; Match emotion versus Attend2; Match gender versus Attend2) at a threshold of $q(F D R)<0.05$. Finally, as the main goal of this study was to investigate the effect of attentional load and spatial location of the emotional stimuli on amygdala responses to unattended fearful and happy faces, I performed a ROI analysis of the amygdala. I conducted a attend-faces (Match emotion and Match gender) versus ignore-faces (Attend1 and Attend2) condition contrast including all experimental runs, whereby a functional ROI of significantly activated voxels was identified (right amygdala: $x, y, z=19$, -6, -9; left amygdala: $\mathrm{x}, \mathrm{y}, \mathrm{z}=-18,-6,-8 ; \mathrm{q}(\mathrm{FDR})<0.05)$. To ascertain the reliability of the attentionalload manipulation, I defined another ROI in the fusiform gyrus, an area known to be implicated in face processing (Kanwisher et al., 1997) and modulated by selective attention (Reddy et al., 2007; Wojciulik et al., 1998). In both hemispheres a cuboid (19x33x16 $\mathrm{mm}^{3}$ ) centred on the mean coordinates of the fusiform gyrus (right fusiform: $x, y, z=35,-55,-$ 15; left fusiform $=-38,-61,-13$ ) obtained from the Brede Database (Nielsen, 2003) was identified. Within these ROIs, I computed the maximum percent signal change averaged across participants for each experimental condition with respect to the spatial locations of the emotional stimuli. Significance was tested using paired t-tests. 


\section{$\underline{6.3 \text { Results }}$}

\subsubsection{Behavioural Results}

Mean reaction times (RTs) for each experimental condition and each subject were computed. First, we examined task effects on RTs considering the spatial location of the emotional faces (Figure 44a). During the ignore-faces conditions, participants responded significantly faster in Attend1 than in Attend2 when the unattended faces were presented in the central $(F(1,13)=20.4, p<0.001)$, middle $(F(1,13)=6.4, p<0.02)$ and peripheral position $(F(1,13)=6.0, p<0.02)$. During the attend-faces conditions, subjects matched the expression significantly faster than the gender of the faces at all three spatial locations (central: $\mathrm{F}(1,13)=39.3, \mathrm{p}<0.001$; middle: $\mathrm{F}(1,13)=163.1, \mathrm{p}<0.001 ;$ peripheral: $\mathrm{F}(1,13)=235.2$, $\mathrm{p}<0.001)$. Within tasks, subjects responded significantly faster in Attend1, when the emotional stimuli were presented in the peripheral than in the central location $(\mathrm{F}(1,13)=4.7, \mathrm{p}<0.05)$. The RTs in Match gender, were also affected by the position of the attended faces in being significantly decreased, when the emotional stimuli were presented in the peripheral compared to the central location $(F(1,13)=5.0, p<0.04)$ and to the middle location $(\mathrm{F}(1,13)=6.4, \mathrm{p}<0.02)$. During Match emotion, participants responded faster, when the faces were located in the middle position compared to the peripheral position $(\mathrm{F}(1,13)=9.4, \mathrm{p}<0.009)$.

In a second step, the efficacy of the attentional-load manipulation was tested by assessing the accuracy of the different experimental conditions (Figure 44b). As expected, mean task performance for all different conditions was well above chance level $(p<0.001)$. Subjects were more accurate in Attend1 than in Attend2, regardless of the face distractor positions (central: $\mathrm{F}(1,13)=22.6, \mathrm{p}<0.001$; middle: $\mathrm{F}(1,13)=17.0, \mathrm{p}<0.001$; peripheral: $\mathrm{F}(1,13)=19.7$, $\mathrm{p}<0.001)$. During attend-faces trials subjects only performed significantly better in Match emotion than in Match gender, when the faces were presented in the peripheral location $(F(1,13)=5.2, p<0.04)$. During Match gender performance was significantly higher during trials, in which the faces were presented at the central position compared to the peripheral position $(\mathrm{F}(1,13)=5.1, \mathrm{p}=0.04)$. Interestingly, subjects were more accurate during Attend2, when the faces were located in the peripheral position rather than in the central $(\mathrm{F}(1,13)=13.4, \mathrm{p}<0.003)$ or in the middle $(\mathrm{F}(1,13)=5.9, \mathrm{p}<0.03)$ position. 
A

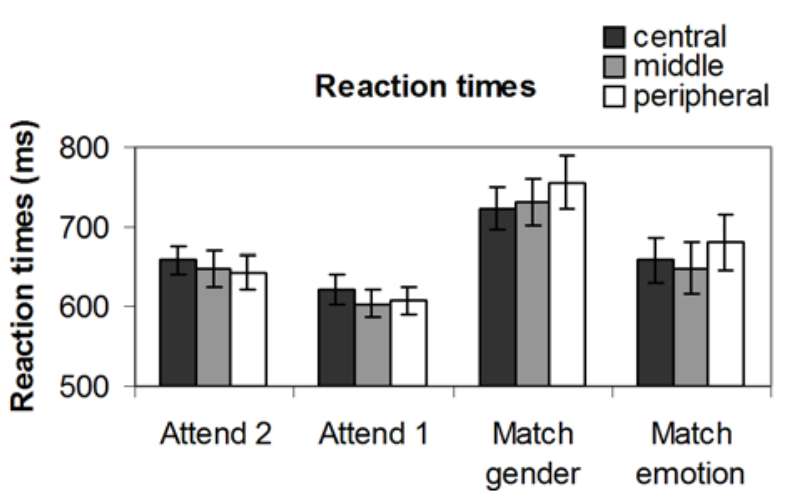

B

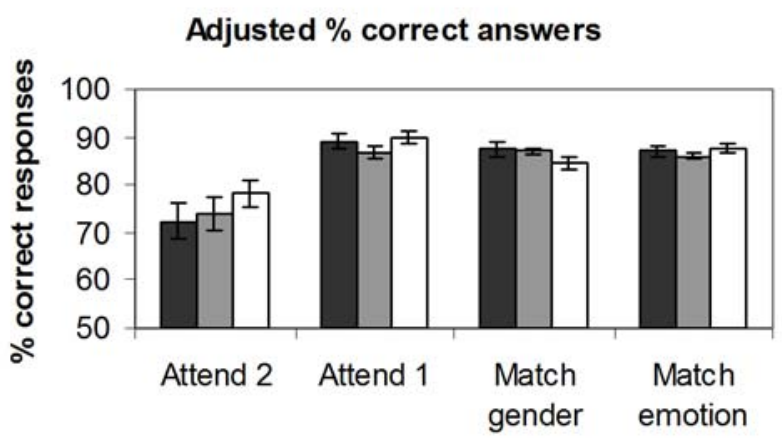

\section{Figure 44}

Behavioural results. (A) Reaction times (mean \pm S.E.) for each task condition as a function of the location of the face stimuli. (B) Adjusted percent correct answers for each task condition in relation to the position of the emotional stimuli. See text for significant differences.

In between scan sessions no significant difference in task performance was observed for the attend-face trials, whereas during Attend1 and Attend2, a significant increase in performance was obtained from the first scan session compared to the third scan session (Attend1: $F(1,13)=7.0, p<0.02$; Attend2: $F(1,13)=5.0, p<0.04)$.

\subsubsection{Neuroimaging Results}

\subsubsection{Main task effects}

Initially, we examined the role of cognitive and emotional modulation by comparing responses evoked by the different task conditions relative to fixation (Figure 45, Table 4). Roughly the same distributed network of brain regions was activated during all different experimental conditions including dorsal anterior cingulate cortex, dorsolateral prefrontal cortex, precentral gyrus, posterior parietal cortex, anterior insula, putamen, midbrain, fusiform gyrus and superior temporal sulcus as well as middle temporal area and temporo- 
parietal junction. However, also deactivations in several regions were observed such as the rostral anterior cingulate coretx, posterior cingulate cortex, cuneus, angular gyrus, superior frontal gyrus, superior temporal gyrus, middle temporal gyrus and posterior insula.

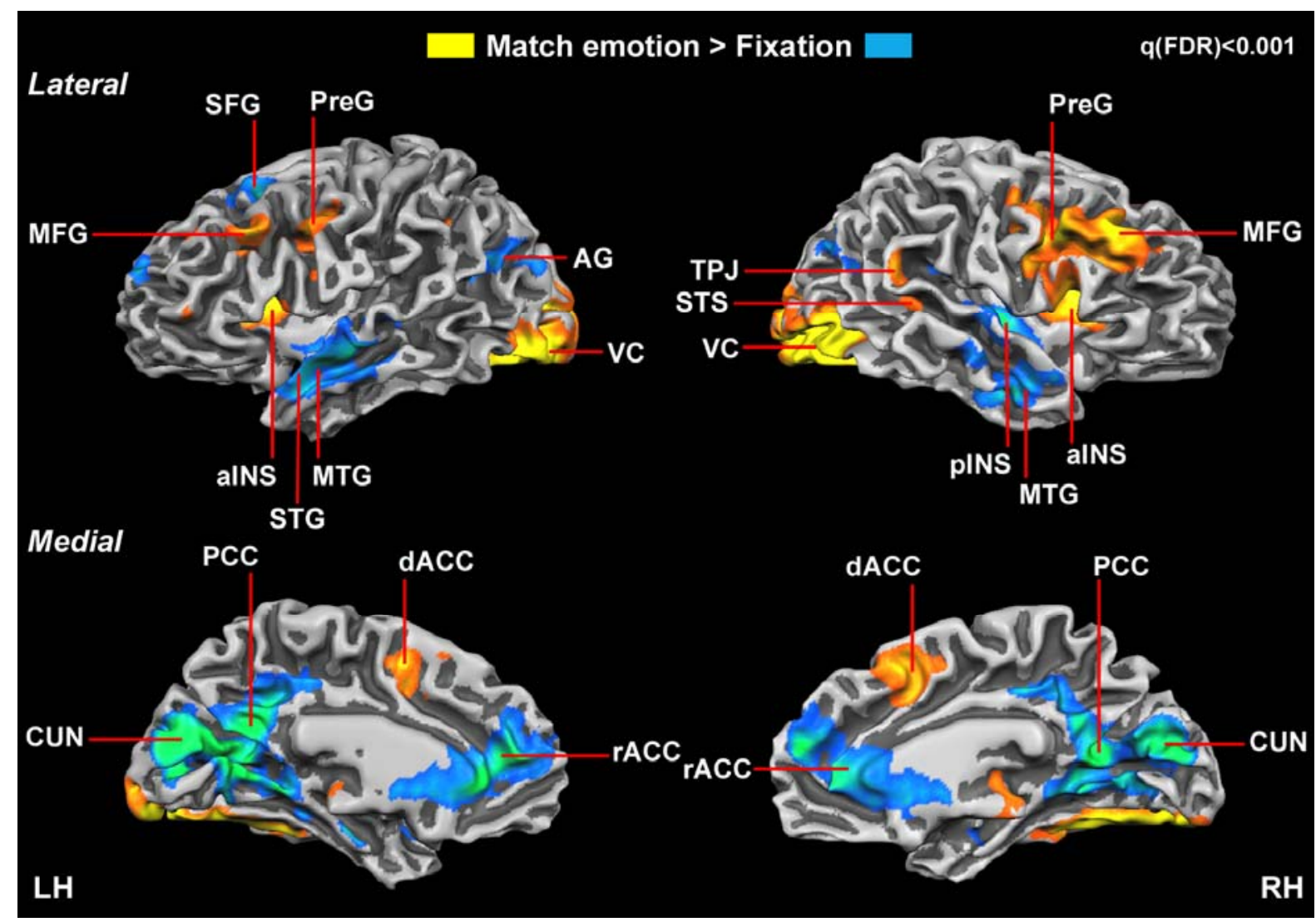

\section{Figure 45}

Lateral and medial view of the surface reconstruction of the MNI template brain (courtesy of the Montreal Neurological Institute) showing the network of brain regions activated during Match emotion (red/yellow) in relation to Fixation (green/blue), when the face stimuli were presented in the central position. LH: left hemisphere; RH: right hemisphere; SFG: superior frontal gyrus; PreG: precentral gyrus; AG: angular gyrus; VC: visual cortex; MTG: middle temporal gyrus; STG: superior temporal gyrus; STS: superior temporal sulcus; aINS: anterior insula; pINS: posterior insula; MFG: middle frontal gyrus; TPJ: temporo-parietal junction; PCC: posterior cingulate cortex; dACC: dorsal anterior cingulate cortex; rACC: rostral anterior cingulate cortex; CUN: cuneus. 
Table 5

Main effects of attend-faces conditions relative to fixation (central position; $\mathrm{p}<0.001$ )

\begin{tabular}{|c|c|c|c|c|c|}
\hline \multirow[b]{2}{*}{ Brain region } & \multirow[b]{2}{*}{ Side } & \multicolumn{3}{|c|}{ Coordinates $^{\mathrm{a}}$} & \multirow[t]{2}{*}{ t-Value ${ }^{b}$} \\
\hline & & $\mathrm{x}$ & $\mathrm{y}$ & $\mathrm{z}$ & \\
\hline \multicolumn{6}{|l|}{ Match emotion > Fixation } \\
\hline \multirow[t]{2}{*}{ Dorsal anterior cingulate cortex } & $\mathrm{R}$ & 3 & 14 & 46 & 8.6 \\
\hline & $\mathrm{L}$ & -6 & 2 & 46 & 9.6 \\
\hline \multirow[t]{2}{*}{ Precentral gyrus } & $\mathrm{R}$ & 42 & -1 & 34 & 17.7 \\
\hline & $\mathrm{L}$ & -39 & -4 & 37 & 9.9 \\
\hline \multirow[t]{2}{*}{ Middle frontal gyrus } & $\mathrm{R}$ & 42 & 26 & 34 & 12.0 \\
\hline & $\mathrm{L}$ & -42 & 23 & 31 & 7.8 \\
\hline \multirow[t]{2}{*}{ Anterior Insula } & $\mathrm{R}$ & 30 & 17 & 13 & 16.7 \\
\hline & $\mathrm{L}$ & -27 & 20 & 10 & 16.8 \\
\hline \multirow[t]{2}{*}{ Putamen } & $\mathrm{R}$ & 21 & 2 & 13 & 13.0 \\
\hline & $\mathrm{L}$ & -24 & -1 & 7 & 11.2 \\
\hline \multirow[t]{2}{*}{ Midbrain } & $\mathrm{R}$ & 9 & -19 & -5 & 9.7 \\
\hline & $\mathrm{L}$ & -6 & -19 & -8 & 8.2 \\
\hline Superior temporal sulcus & $\mathrm{R}$ & 45 & -43 & 10 & 6.1 \\
\hline Inferior temporal gyrus & $\mathrm{R}$ & 42 & -67 & 1 & 18.1 \\
\hline \multirow[t]{2}{*}{ Inferior parietal lobule } & $\mathrm{R}$ & 42 & -43 & 34 & 5.7 \\
\hline & $\mathrm{L}$ & -42 & -37 & 40 & 6.9 \\
\hline \multirow[t]{2}{*}{ Inferior parietal sulcus } & $\mathrm{R}$ & 33 & -55 & 37 & 5.0 \\
\hline & $\mathrm{L}$ & -30 & -52 & 34 & 5.4 \\
\hline Temporo-parietal junction & $\mathrm{R}$ & 57 & -40 & 22 & 7.7 \\
\hline \multicolumn{6}{|l|}{ Match emotion < Fixation } \\
\hline Rostral anterior cingulate cortex & - & -3 & 35 & 4 & -12.1 \\
\hline \multirow{2}{*}{ Posterior cingulate cortex } & $\mathrm{R}$ & 6 & -58 & 16 & -22.7 \\
\hline & $\mathrm{L}$ & -12 & -61 & 13 & -15.7 \\
\hline Superior frontal gyrus & $\mathrm{L}$ & -18 & 17 & 46 & -7.5 \\
\hline \multirow[t]{2}{*}{ Posterior Insula } & $\mathrm{R}$ & 36 & -13 & 4 & -11.9 \\
\hline & $\mathrm{L}$ & -42 & -16 & 1 & -8.1 \\
\hline Superior temporal gyrus & $\mathrm{L}$ & -51 & 13 & -11 & -8.9 \\
\hline Middle temporal gyrus & $\mathrm{R}$ & 48 & -10 & -17 & -10.1 \\
\hline Cuneus & $\mathrm{L}$ & -6 & -79 & 19 & -14.7 \\
\hline \multirow[t]{2}{*}{ Parahippocampal gyrus } & $\mathrm{R}$ & 15 & -16 & -14 & -6.3 \\
\hline & $\mathrm{L}$ & -24 & -19 & -14 & -8.0 \\
\hline Angular gyrus & $\mathrm{L}$ & -45 & -55 & 25 & -6.7 \\
\hline \multicolumn{6}{|l|}{ Match gender $>$ Fixation } \\
\hline \multirow[t]{2}{*}{ Dorsal anterior cingulate cortex } & $\mathrm{R}$ & 9 & 11 & 38 & 6.6 \\
\hline & $\mathrm{L}$ & -12 & 5 & 43 & 10.8 \\
\hline \multirow[t]{2}{*}{ Precentral gyrus } & $\mathrm{R}$ & 39 & -1 & 34 & 17.5 \\
\hline & $\mathrm{L}$ & -39 & -4 & 37 & 9.7 \\
\hline Middle frontal gyrus & $\mathrm{R}$ & 42 & 26 & 34 & 7.4 \\
\hline \multirow[t]{2}{*}{ Anterior Insula } & $\mathrm{R}$ & 30 & 17 & 13 & 14.3 \\
\hline & $\mathrm{L}$ & -27 & 11 & 16 & 19.3 \\
\hline Putamen & $\mathrm{R}$ & 21 & 2 & 13 & 10.8 \\
\hline & $\mathrm{L}$ & -21 & 5 & 10 & 9.4 \\
\hline Thalamus & $\mathrm{R}$ & 6 & -22 & -2 & 8.3 \\
\hline & $\mathrm{L}$ & -6 & -22 & 1 & 8.5 \\
\hline Inferior temporal gyrus & $\mathrm{R}$ & 42 & -67 & 1 & 13.0 \\
\hline Inferior parietal lobule & $\mathrm{R}$ & 39 & -40 & 40 & 5.3 \\
\hline & $\mathrm{L}$ & -42 & -37 & 40 & 6.8 \\
\hline
\end{tabular}




\begin{tabular}{llrrrr} 
Inferior parietal sulcus & $\mathrm{R}$ & 24 & -67 & 25 & 7.3 \\
Temporo-parietal junction & $\mathrm{L}$ & -27 & -55 & 31 & 5.1 \\
& $\mathrm{R}$ & 57 & -43 & 22 & 6.3 \\
Match gender < Fixation & & & & & \\
\hline Rostral anterior cingulate cortex & - & 1 & 38 & 7 & -10.3 \\
Posterior cingulate cortex & $\mathrm{R}$ & 6 & -58 & 16 & -21.7 \\
& $\mathrm{~L}$ & -12 & -64 & 16 & -17.5 \\
Superior frontal gyrus & $\mathrm{L}$ & -18 & 20 & 52 & -8.2 \\
Posterior Insula & $\mathrm{R}$ & 36 & -10 & 4 & -9.8 \\
& $\mathrm{~L}$ & -42 & -16 & 4 & -7.7 \\
Superior temporal gyrus & $\mathrm{R}$ & 48 & 8 & -17 & -11.8 \\
& $\mathrm{~L}$ & -48 & 8 & -17 & -8.1 \\
Cuneus & $\mathrm{L}$ & -9 & -82 & 19 & -15.0 \\
Parahippocampal gyrus & $\mathrm{R}$ & 24 & -10 & -11 & -6.5 \\
& $\mathrm{~L}$ & -24 & -16 & -14 & -7.8 \\
Angular gyrus & $\mathrm{L}$ & -42 & -49 & 22 & -7.6
\end{tabular}

Main effects of ignore-faces conditions relative to fixation (central position; $\mathrm{p}<0.001$ )

\begin{tabular}{|c|c|c|c|c|c|}
\hline \multirow[b]{2}{*}{ Brain region } & \multirow[b]{2}{*}{ Side } & \multicolumn{3}{|c|}{ Coordinates $^{\mathrm{a}}$} & \multirow[t]{2}{*}{ t-Value ${ }^{b}$} \\
\hline & & $\mathrm{x}$ & $\mathrm{y}$ & $\mathrm{z}$ & \\
\hline \multicolumn{6}{|l|}{ Attend1 > Fixation } \\
\hline \multirow[t]{2}{*}{ Dorsal anterior cingulate cortex } & $\mathrm{R}$ & 6 & 17 & 37 & 7.9 \\
\hline & $\mathrm{L}$ & -12 & 2 & 40 & 9.3 \\
\hline \multirow[t]{2}{*}{ Precentral gyrus } & $\mathrm{R}$ & 39 & -4 & 33 & 12.0 \\
\hline & $\mathrm{L}$ & -39 & -4 & 34 & 7.0 \\
\hline \multirow[t]{2}{*}{ Middle frontal gyrus } & $\mathrm{R}$ & 27 & 26 & 28 & 6.5 \\
\hline & $\mathrm{L}$ & -36 & 29 & 34 & 5.3 \\
\hline \multirow[t]{2}{*}{ Anterior Insula } & $\mathrm{R}$ & 28 & 17 & 13 & 15.2 \\
\hline & $\mathrm{L}$ & -30 & 11 & 16 & 17.0 \\
\hline \multirow[t]{2}{*}{ Putamen } & $\mathrm{R}$ & 28 & 11 & 13 & 12.4 \\
\hline & $\mathrm{L}$ & -21 & 5 & 7 & 12.7 \\
\hline \multirow[t]{2}{*}{ Midbrain } & $\mathrm{R}$ & 6 & -22 & -2 & 8.6 \\
\hline & $\mathrm{L}$ & -6 & -22 & -2 & 8.0 \\
\hline Inferior temporal gyrus & $\mathrm{R}$ & 42 & -67 & -1 & 16.6 \\
\hline \multirow{2}{*}{ Inferior parietal lobule } & $\mathrm{R}$ & 39 & -40 & 34 & 7.1 \\
\hline & $\mathrm{L}$ & -42 & -40 & 37 & 8.3 \\
\hline \multirow{2}{*}{ Inferior parietal sulcus } & $\mathrm{R}$ & 24 & -70 & 25 & 9.0 \\
\hline & $\mathrm{L}$ & -27 & -52 & 40 & 5.2 \\
\hline Temporo-parietal junction & $\mathrm{R}$ & 57 & -43 & 22 & 10.1 \\
\hline \multicolumn{6}{|l|}{ Attend1 $<$ Fixation } \\
\hline Rostral anterior cingulate cortex & - & 2 & 47 & 10 & -11.9 \\
\hline \multirow[t]{2}{*}{ Posterior cingulate cortex } & $\mathrm{R}$ & 6 & -53 & 7 & -15.3 \\
\hline & $\mathrm{L}$ & -12 & -55 & 7 & -14.0 \\
\hline Posterior Insula & $\mathrm{R}$ & 39 & -10 & 4 & -8.3 \\
\hline \multirow[t]{2}{*}{ Middle temporal gyrus } & $\mathrm{R}$ & 54 & -1 & -17 & -8.6 \\
\hline & $\mathrm{L}$ & -54 & -7 & -8 & -10.6 \\
\hline Cuneus & - & 0 & -72 & 19 & -15.9 \\
\hline \multirow[t]{2}{*}{ Parahippocampal gyrus } & $\mathrm{R}$ & 29 & -10 & -14 & -5.3 \\
\hline & $\mathrm{L}$ & -24 & -16 & -14 & -7.5 \\
\hline \multirow[t]{2}{*}{ Angular gyrus } & $\mathrm{R}$ & 42 & -67 & 28 & -6.5 \\
\hline & $\mathrm{L}$ & -51 & -64 & 22 & -6.5 \\
\hline
\end{tabular}




\begin{tabular}{|c|c|c|c|c|c|}
\hline \multirow{2}{*}{ Dorsal anterior cingulate cortex } & $\mathrm{R}$ & 3 & 14 & 46 & 8.9 \\
\hline & $\mathrm{L}$ & -6 & 5 & 49 & 9.6 \\
\hline \multirow[t]{2}{*}{ Precentral gyrus } & $\mathrm{R}$ & 39 & -4 & 31 & 14.7 \\
\hline & $\mathrm{L}$ & -39 & -1 & 34 & 9.1 \\
\hline \multirow[t]{2}{*}{ Middle frontal gyrus } & $\mathrm{R}$ & 39 & 26 & 34 & 12.8 \\
\hline & $\mathrm{L}$ & -39 & 26 & 34 & 8.3 \\
\hline \multirow[t]{2}{*}{ Anterior Insula } & $\mathrm{R}$ & 28 & 17 & 13 & 14.4 \\
\hline & $\mathrm{L}$ & -30 & 11 & 16 & 16.8 \\
\hline \multirow[t]{2}{*}{ Putamen } & $\mathrm{R}$ & 15 & 2 & 13 & 11.7 \\
\hline & $\mathrm{L}$ & -18 & -4 & 16 & 12.8 \\
\hline \multirow[t]{2}{*}{ Midbrain } & $\mathrm{R}$ & 6 & -22 & -2 & 9.7 \\
\hline & $\mathrm{L}$ & -6 & -22 & -2 & 9.0 \\
\hline \multirow[t]{2}{*}{ Inferior temporal gyrus } & $\mathrm{R}$ & 42 & -70 & 1 & 19.7 \\
\hline & $\mathrm{L}$ & -42 & -73 & -1 & 9.3 \\
\hline \multirow[t]{2}{*}{ Inferior parietal lobule } & $\mathrm{R}$ & 36 & -37 & 34 & 6.3 \\
\hline & $\mathrm{L}$ & -42 & -43 & 40 & 6.4 \\
\hline \multirow[t]{2}{*}{ Inferior parietal sulcus } & $\mathrm{R}$ & 24 & -70 & 25 & 12.6 \\
\hline & $\mathrm{L}$ & -27 & -52 & 37 & 5.1 \\
\hline Temporo-parietal junction & $\mathrm{R}$ & 57 & -43 & 22 & 10.2 \\
\hline \multicolumn{6}{|l|}{ Attend $2<$ Fixation } \\
\hline Rostral anterior cingulate cortex & - & 3 & 44 & 13 & -9.3 \\
\hline \multirow[t]{2}{*}{ Posterior cingulate cortex } & $\mathrm{R}$ & 6 & -55 & 10 & -19.9 \\
\hline & $\mathrm{L}$ & -15 & -61 & 16 & -12.9 \\
\hline Posterior Insula & $\mathrm{R}$ & 36 & -13 & 4 & -7.0 \\
\hline \multirow[t]{2}{*}{ Middle temporal gyrus } & $\mathrm{R}$ & 48 & -1 & -14 & -7.3 \\
\hline & $\mathrm{L}$ & -54 & -2 & -8 & -8.5 \\
\hline Cuneus & $\mathrm{L}$ & -3 & -73 & 19 & -14.4 \\
\hline \multirow[t]{2}{*}{ Parahippocampal gyrus } & $\mathrm{R}$ & 24 & -10 & -8 & -6.1 \\
\hline & $\mathrm{L}$ & -24 & -19 & -11 & -7.4 \\
\hline \multirow[t]{2}{*}{ Angular gyrus } & $\mathrm{R}$ & 42 & -67 & 28 & -7.1 \\
\hline & $\mathrm{L}$ & -42 & -64 & 25 & -7.4 \\
\hline
\end{tabular}

a Talairach coordinates in $\mathrm{mm}$.

$\mathrm{b}$ t-Value of the peak-activity.

\subsubsection{Attend-faces versus ignore-faces conditions}

In concordance with faces being task-relevant during Match emotion and Match gender, respective signal changes relative to Attend1 and Attend2 were observed in the fusiform gyrus and amygdala, when the faces were presented in the central location (Table 5, Figure 46). Attending the faces in the middle position was associated with differential responses in the amygdala and fusiform gyrus in contrast to Attend1, whereas Match emotion compared to Attend2 revealed signal changes only in the amygdala. With face stimuli in the peripheral position, signal changes in the amygdala and fusiform gyrus were found by contrasting Match gender versus Attend1, while Match emotion versus Attend1 only resulted in increased activity in the fusiform gyrus. 
Table 6

Activations in the amygdala and fusiform gyrus $(\mathrm{p}<0.05)$

\begin{tabular}{|c|c|c|c|c|c|c|}
\hline \multirow[b]{2}{*}{ Position $^{\mathrm{a}}$} & \multirow[b]{2}{*}{ Brain region } & \multirow[b]{2}{*}{ Side } & \multicolumn{2}{|c|}{ Coordinates $^{\mathrm{b}}$} & & \multirow[t]{2}{*}{ t-Value ${ }^{c}$} \\
\hline & & & $\mathrm{x}$ & $\mathrm{y}$ & & \\
\hline \multicolumn{7}{|c|}{ Match emotion > Attend1 } \\
\hline \multirow[t]{4}{*}{ Central } & Amygdala & $\mathrm{R}$ & 18 & -4 & -11 & 9.2 \\
\hline & & $\mathrm{L}$ & -15 & -7 & -8 & 6.7 \\
\hline & Fusiform gyrus & $\mathrm{R}$ & 25 & -43 & -14 & 5.6 \\
\hline & & $\mathrm{L}$ & -30 & -76 & -17 & 10.0 \\
\hline \multirow[t]{4}{*}{ Middle } & Amygdala & $\mathrm{R}$ & 18 & -7 & -8 & 7.9 \\
\hline & & $\mathrm{L}$ & -18 & -7 & -8 & 10.5 \\
\hline & Fusiform gyrus & $\mathrm{R}$ & 39 & -46 & -17 & 6.4 \\
\hline & & $\mathrm{L}$ & -30 & -73 & -14 & 9.4 \\
\hline Peripheral & Fusiform gyrus & $\mathrm{L}$ & -30 & -46 & -11 & 8.3 \\
\hline \multicolumn{7}{|c|}{ Match gender $>$ Attend1 } \\
\hline \multirow[t]{3}{*}{ Central } & Amygdala & $\mathrm{R}$ & 15 & -7 & -8 & 12.2 \\
\hline & & $\mathrm{L}$ & -21 & -4 & -8 & 6.5 \\
\hline & Fusiform gyrus & $\mathrm{R}$ & 27 & -43 & -27 & 6.5 \\
\hline \multirow[t]{3}{*}{ Middle } & Amygdala & $\mathrm{R}$ & 18 & -7 & -8 & 7.4 \\
\hline & & $\mathrm{L}$ & -18 & -7 & -8 & 11.4 \\
\hline & Fusiform gyrus & $\mathrm{R}$ & 25 & -40 & -11 & 6.0 \\
\hline \multirow[t]{4}{*}{ Peripheral } & Amygdala & $\mathrm{R}$ & 18 & -7 & -8 & 8.0 \\
\hline & & $\mathrm{L}$ & -18 & -7 & -8 & 12.4 \\
\hline & Fusiform gyrus & $\mathrm{R}$ & 27 & -46 & -17 & 5.7 \\
\hline & & $\mathrm{L}$ & -30 & -55 & -14 & 8.9 \\
\hline
\end{tabular}

Match emotion $>$ Attend2

\begin{tabular}{lllrrrr}
\hline Central & Amygdala & $\mathrm{R}$ & 15 & -4 & -11 & 6.6 \\
& Fusiform gyrus & $\mathrm{R}$ & 28 & -43 & -17 & 4.6 \\
Middle & Amygdala & $\mathrm{R}$ & 18 & -7 & -8 & 8.4 \\
& & $\mathrm{~L}$ & -18 & -1 & -8 & 6.5
\end{tabular}

Match gender $>$ Attend2

\begin{tabular}{lllllrl}
\hline Central & Amygdala & $\mathrm{R}$ & 24 & -13 & -8 & 6.4 \\
& Fusiform gyrus & $\mathrm{R}$ & 28 & -43 & -17 & 6.4 \\
\hline
\end{tabular}

a Position of the face stimuli in the visual field.

$\mathrm{b}$ Talairach coordinates in $\mathrm{mm}$.

c $\mathrm{t}$-Value of the peak-activity. 


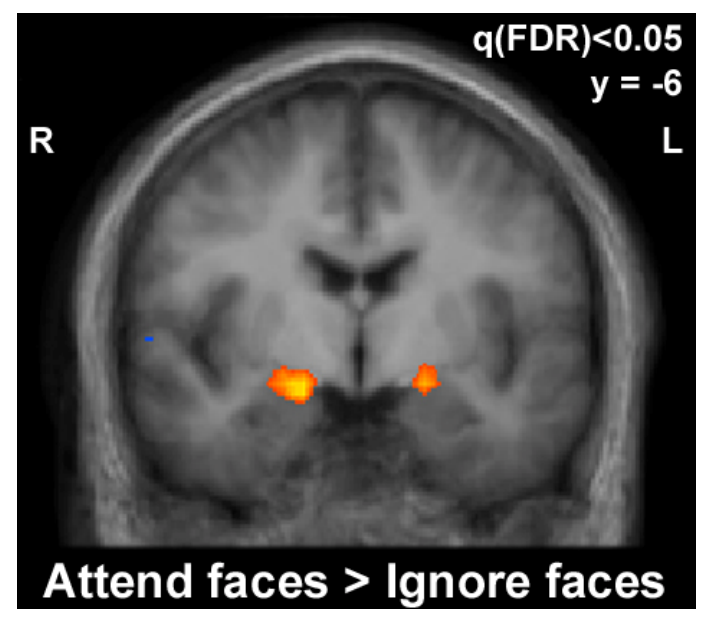

\section{Figure 46}

Averaged anatomical T1-weighted coronal section through the amygdala and group activations (red) of the contrast of the attend-faces tasks (Match gender+Match emotion) versus the ignorefaces tasks $($ Attend1+2). Face stimuli were located in the central position. L: left; R: right.

\subsubsection{Amygdala region of interest analysis}

We investigated the effect of attentional load and the spatial position of the emotional stimuli on amygdala activity by performing an ROI analysis. Figure 47 plots the average percent signal change for all task conditions as a function of the position of the faces (central, middle, peripheral). First, we analyzed each ROI for the left and right amygdala separately, but as no significant differences in signal changes were observed between them, both amygdalae were summarized in one ROI.

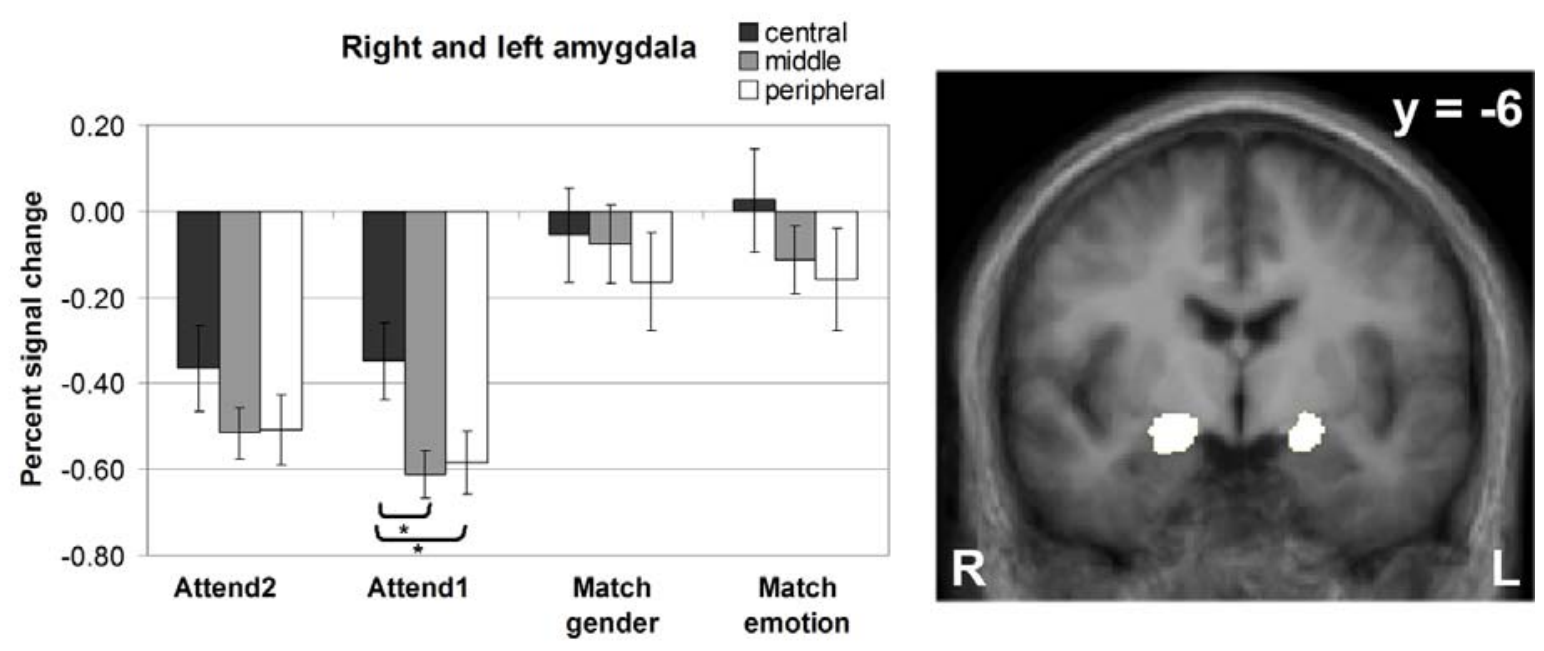

\section{Figure 47}

Mean percent signal changes ( \pm S.E. $)$ in right and left amygdala during the Attend2, Attend1, Match gender and Match emotion task in relation to the spatial location of the face stimuli (left) $\left({ }^{*} \mathrm{p}<0.05\right)$. See text for between task comparisons. The same amygdala ROI (white) was used for all participants (right). L: left; R: right. 
Overall, suppressed amygdala responses were observed during all task conditions. Amygdala responses during Attend1 and Attend2 were largely decreased. Signal decreases were also obtained during the attend-faces conditions. Between Attend1 and Attend2, as well as between Match emotion and Match gender, no significant differences in amygdala responses were found. Comparing the signal changes between Attend1 and Match emotion revealed a significantly larger decrease in trials, when the faces were unattended and presented in the central position $(\mathrm{F}(1,13)=22.3, \mathrm{p}<0.001)$, in the middle position $(\mathrm{F}(1,13)=108.2, \mathrm{p}<0.001)$ and in the peripheral position $(\mathrm{F}(1,13)=40.7, \mathrm{p}<0.001)$. Corresponding results were observed comparing Attend1 with Match gender (central: $\mathrm{F}(1,13)=11.3, \mathrm{p}<0.005 ;$ middle: $\mathrm{F}(1,13)=80.7, \mathrm{p}<0.001 ;$ peripheral: $\mathrm{F}(1,13)=41.1$, $\mathrm{p}<0.001)$. Attend 2 compared to Match emotion produced significant more signal decreases regardless of the position of the face stimuli (central: $F(1,13)=23.1, p<0.001$; middle: $\mathrm{F}(1,13)=22.1, \mathrm{p}<0.001$; peripheral: $\mathrm{F}(1,13)=15.2, \mathrm{p}<0.002)$. A significantly decreased signal was also found comparing Attend2 with Match gender in all spatial positions of the emotional stimuli (central: $\mathrm{F}(1,13)=14.2, \mathrm{p}<0.002$; middle: $\mathrm{F}(1,13)=22.1, \mathrm{p}<0.001$; peripheral: $\mathrm{F}(1,13)=17.2, \mathrm{p}<0.001)$.

Within tasks, a significant difference in signal change was only observed during Attend1, comparing conditions of central face presentation with the middle $(F(1,13)=7.6, p<0.02)$ and peripheral $(\mathrm{F}(1,13)=6.0, \mathrm{p}<0.03)$ location of the emotional stimuli.

\subsubsection{Fusiform gyrus region of interest analysis}

The mean percent signal change was investigated within bilateral fusiform gyri for all tasks as a function of the location of the emotional stimuli (Figure 48). Comparing the signal change between Attend1 and Match emotion revealed a significant increase in activity for Match emotion, when the faces were located in the central position $(F(1,13)=6.3, p<0.03)$, in the middle position $(\mathrm{F}(1,13)=18.8, \mathrm{p}<0.001)$, and in the peripheral position $(F(1,13)=10.4, p<0.007)$. Similar results were obtained with greater activations when subjects matched the gender of the faces in the central $(F(1,13)=6.2, p<0.03)$, middle $(F(1,13)=21.5, p<0.001)$ and peripheral $(F(1,13)=12.7, p<0.003)$ location rather than when they performed Attend1. The comparison between Attend2 and Match emotion indicated a higher signal change for Match emotion, when the task-relevant faces were displayed in the peripheral position $(\mathrm{F}(1,13)=6.3, \mathrm{p}<0.03)$. When subjects matched the gender of the faces, a significant signal increase was obtained compared to Attend 2 when the emotional stimuli 
were presented centrally $(\mathrm{F}(1,13)=6.1, \mathrm{p}<0.03)$. Furthermore, Attend2 revealed a significant increase in activity in contrast to Attend1, when the to-be-ignored stimuli were presented in the middle location $(\mathrm{F}(1,13)=11.9, \mathrm{p}<0.004)$.

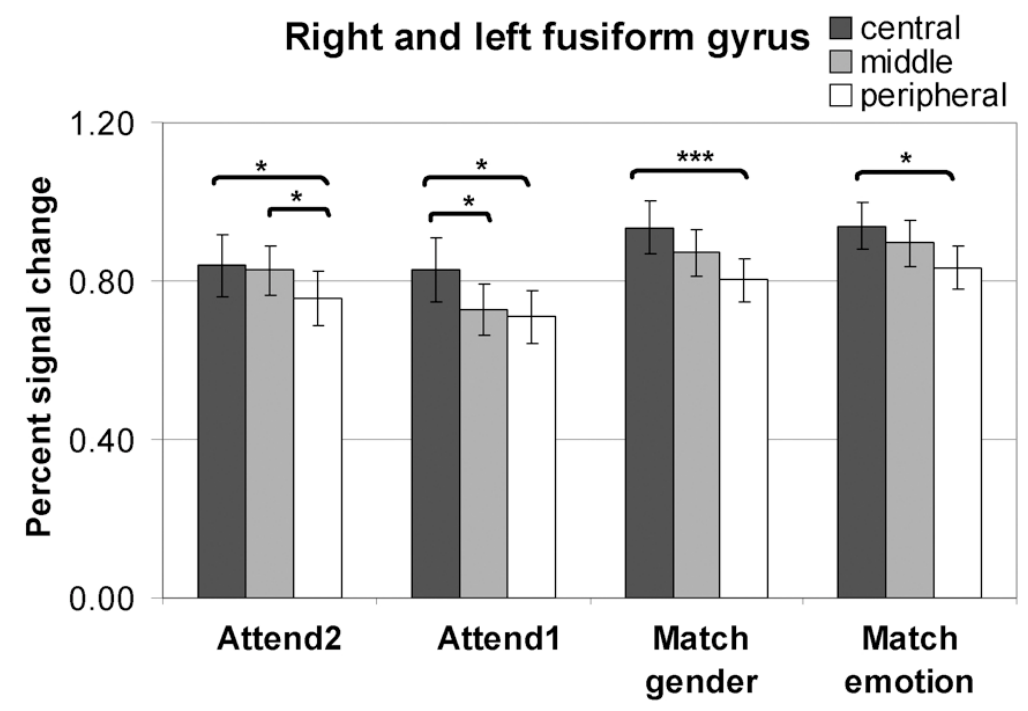

\section{Figure 48}

Mean percent signal changes ( \pm S.E.) in right and left fusiform gyrus during the Attend2, Attend1, Match gender and Match emotion task in relation to the spatial location of the face stimuli $\left({ }^{*} \mathrm{p}<0.05 ; * * * \mathrm{p}<0.001\right)$. See text for between task comparisons. L: left; R: right.

Within task conditions, a linear increase of activation in the fusiform gyrus was observed with decreasing distance between the faces. The location of the unattended faces affected the signal change during Attend1 task by producing a significant increase in activity, when the emotional stimuli were positioned in the central location compared to the middle $(\mathrm{F}(1,13)=5.0, \mathrm{p}<0.05)$ and the peripheral $(\mathrm{F}(1,13)=5.8, \mathrm{p}<0.04)$ location. During Attend2, enhanced responses in the fusiform gyri were found, when the to-be-ignored stimuli were positioned in the central $(\mathrm{F}(1,13)=5.5, \mathrm{p}<0.04)$ and middle $(\mathrm{F}(1,13)=5.1, \mathrm{p}<0.05)$ location compared to the peripheral one. When the subjects directed their attention to the faces by matching the expression and the gender in the central loaction rather than in the peripheral location, a significant increase in activity was observed (Match emotion, central: $\mathrm{F}(1,13)=6.6, \mathrm{p}<0.03 ;$ Match gender, central: $\mathrm{F}(1,13)=16.3, \mathrm{p}<0.001)$. 


\section{$\underline{\text { 6.4 Discussion }}$}

In the present study, we investigated the impact of attentional load and the effect of spatial location of the emotional stimuli on face processing in the amygdala. We designed an experiment, which allowed us to directly assess the interaction between these two factors within the same group of participants. During all task conditions a decrease in signal intensity in the amygdala was observed indicating that amygdala responses to emotional stimuli are strongly resource-dependent and are completely suppressed during conditions, which efficiently exhaust attentional resources.

The attentional-load manipulation in the current experiment was validated by the behavioural results. Our findings show that subjects responded faster and more accurately during Attend1 than Attend2 indicating that the split of attention among two spatial positions in opposing quadrants of the visual field induces an increase in task difficulty and is more attentionally demanding (high attentional load), which is line with previous results (Morawetz et al., 2007). To keep performance of foveal and peripheral face recognition at a comparable level, image size was increased towards the visual field periphery. Indeed, during the attend-faces conditions mean task performance was retained with increasing eccentricity and not affected by the location of the face stimuli. Furthermore, there was no difference between Match emotion and Match gender. These results may be taken as an indication that difficulty was approximately equivalent in each task, when response accuracy alone is considered. However, the analysis of the RTs revealed that expression matching was significantly faster than gender classifications, suggesting that expression is more easily discriminable than gender for these stimuli.

\subsubsection{Amygdala}

The present findings of a variable suppression of amygdala response to attended and unattended faces are in direct contrast to studies supporting the notion that attentional-load manipulations do not affect amygdala activity and that the amygdala responds to emotional stimuli regardless of attentional resources available (Vuilleumier et al., 2002; Anderson et al., 2003; Williams et al., 2005). The overall decrease in neuronal activity in the amygdala during the emotional and non-emotional task conditions is concordant with the view that cognitive and emotional systems engage each other in a mutually suppressive way such that, when either system is recruited, the other will be relatively suppressed (Drevets \& 
Raichle, 1998). Thus, an active task may inhibit an area that would normally respond in the task environment during a passive condition. The different visual discrimination tasks in the present study may have been cognitively demanding in such an extent that limbic regions have been inhibited. Indeed, deactivation of the amygdala has been observed in several tasks that involve higher cognitive processing (Devrets \& Raichle, 1995; Shulman et al., 1997). It could be demonstrated that active visual tasks (i.e. attentionally demanding tasks in which subjects make decisions regarding various visual stimuli) relative to passive viewing tasks are associated with decreases in neuronal activity in the amygdala (Shulman et al., 1997). Furthermore, a decrease in amygdala response was found during highattentional load tasks (without emotional faces being present in the visual display) compared to fixation (Pessoa et al., 2005) and compared to a task specific baseline (Hsu \& Pessoa, 2007). On the contrary, the studies supporting the notion of an automatic responsitivity of the amygdala to fearful faces did not vary the attentional load of the different task conditions. The subjects had to direct their attention either to faces or houses matching their identity (Vuilleumier et al., 2001; Williams et al., 2005) or perform a male/female judgement on the faces and an inside/outside judgement on the houses (Anderson et al., 2003). Therefore, the observed decrease in activity may reflect an inhibition of the amygdala response during attentionally demanding tasks and cognitive processing.

Although, the neuronal activity in the amygdala was decreased in the current experiment in all tasks, an influence of attentional-load manipulations was observed. During the explicit processing of the faces (Match gender and Match emotion) the signal changes in the amygdala were decreased to a lesser extent than when the faces were ignored and attention was directed to the letters and digits (Attend1 and Attend2). Our results are therefore in line with previous studies demonstrating discriminative responses of the amygdala to attended and unattended faces (Pessoa et al., 2002; 2005; Mitchell et al., 2007; Bishop et al., 2007; Silvert et al., 2007). Furthermore, a decrease in amygdala activity has been documented when faces of different emotional valence were unattended (Pessoa et al., 2002) and when a demanding task of high attentional load was performed (Pessoa et al., 2005; Mitchell et al., 2007; Bishop et al., 2007), which is in agreement with the present findings.

All above mentioned studies (Pessoa et al., 2002; 2005; Mitchell et al., 2007; Bishop et al., 2007; Anderson et al., 2003) presented the emotional stimuli in the centre of the visual field. However, it has been suggested that the spatial location of the emotional stimuli constitutes an important factor in determining differential amygdala activation (Palermo \& 
Rhodes, 2007). To date, only one fMRI study tested the impact of attentional load on the processing of peripherally presented faces (Silvert et al., 2007). The differential amygdala response to emotional versus neutral unattended faces vanished with increasing attentional load, supporting a resource-dependent activation model of the amygdala responsiveness. However, in contrast to our results, no complete suppression of amygdala activation was observed, when the faces were unattended in the high attentional load task. At this point, we would like to emphasize that our study may not only differ from previous ones in the spatial location of the presented face stimuli, but also in the attentional load of the tasks used to direct attention away from the faces. It has been proposed that complete suppression of amygdala responses to unattended faces may occur only, when the competing tasks exhaust the attentional resources available leaving almost no capacities for processing task-irrelevant stimuli (Pessoa et al., 2005). Thus, as outlined above, we suggest that in the present study both attentional load manipulations were extremely efficient in diverting attention from the faces thereby inducing a massive suppression of neuronal activity in the amygdala. This impact on the amygdala response is supported by the fact that Attend1 as well as Attend2 resulted in a comparable decreased activation level although the behavioural results indicated that Attend2 was of higher attentional load than Attend1.

The design of the present study aimed to investigate the effect of the spatial location of the face stimuli on amygdala response by presenting faces at three different positions in the visual field. Only during one condition (Attend1) the spatial position of the faces affected the signal changes in the amygdala significantly. However, there seems to be a trend that emotional stimuli presented in the central position lead to a less reduced amygdala response than when the faces are presented more peripherally like in the middle and peripheral position regardless of the task condition. Therefore, the idea that the amygdala may be especially sensitive to affective stimuli presented in the periphery could not be supported. On the contrary, we suggest that with increasing task difficulty and increasing distance between the face stimuli, the neuronal activity in the amygdala decreases (Figure 49). 


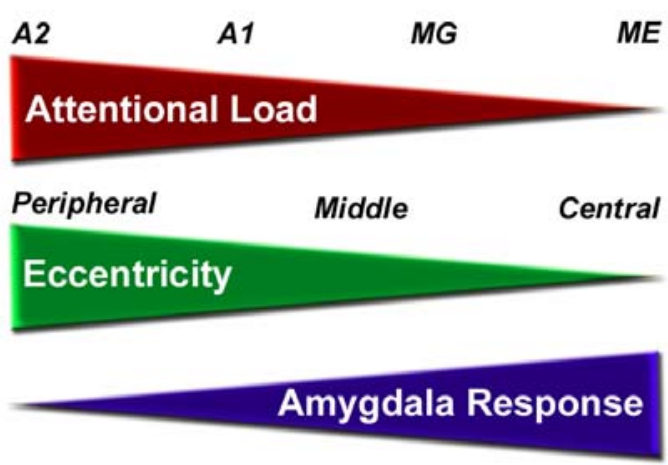

\section{Figure 49}

Schematic of amygdala response subject to attentional load and stimulus position. With increasing attentional load and eccentricity, the amygdala response decreases. A2: Attend2; A1: Attend1; MG: Match gender; ME: Match emotion.

One potential caveat of the present study might be an instability of amygdala responses to facial expressions during multiple imaging sessions over extended periods of time. The reproducibility of fMRI results depends upon a number of variables. Of particular relevance to studies of amygdala activation to emotional faces is the subject's anxiety at the time of the examination, which has also been shown to interact with manipulations of perceptual load to determine the neuronal processing of task-irrelevant fearful faces (Bishop et al., 2007). Further variability in amygdala activity between imaging sessions is likely to result from learning related to experimental task and stimuli (e.g. habituation) (Fischer et al., 2003; Wright et al., 2001; Breiter et al., 1996; Phillips et al., 2001). Most of these studies investigated within-session effects, rather than effects over multiple examinations. In the present study each of the three scan sessions was separated by one week. Therefore, as Johnstone et al. (2005) demonstrated a robust signal change in the amygdala in response to passively perceived fearful faces over a time period of eight weeks, we render an impact of multiple imaging sessions in our study rather unlikely.

\subsubsection{Fusiform gyrus}

Additionally to the amygdala another ROI in the fusiform cortex was defined, which was associated with differential activity induced by the different tasks thereby pointing towards an attentional modulation within this ROI. The involvement of the fusiform gyrus in the processing of faces has been established by numerous imaging studies demonstrating greater activity to faces than to nonsense (control) or non-face stimuli (Kanwisher et al., 1997; McCarthy et al., 1997; Halgren et al., 1999; Haxby et al., 1994, 1999; Hoffman \& 
Haxby, 2000; Ishai et al., 1999; Sergent et al., 1992; Puce et al., 1996). In agreement with several other fMRI studies we observed a significant increase in activity in the fusiform gyrus when face stimuli appeared inside the focus of attention (Silvert et al., 2007; Pessoa et al., 2002; Vuilleumier et al., 2001; Williams et al., 2005; Wojciulik et al., 1998; Reddy et al., 2007; O’Craven et al., 1999). Since attention was manipulated in a blocked design and faces were presented on each trial in the attend-faces and ignore-faces conditions, we also found increased responses to unattended faces in the fusiform cortex consistent with the results of Silvert et al. (2007). Activation in fusiform gyrus was significantly stronger in response to central face stimuli compared to peripheral stimuli, as shown before (Levy et al., 2001; Liu \& Ioannides, 2006). As the activity in the fusiform gyrus was modulated realiabely by selective attention, we presume the observed effects of attentional modulation in the amygdala to be corroborated.

\subsubsection{Whole-brain analysis}

The whole-brain analysis contrasting the different task conditions with the fixation condition revealed activations in a network of brain regions including areas involved in attention-demanding control processes like dACC (Posner, 1995; Posner \& Petersen, 1999; Frith, 2001; Bush et al., 2000; Devrets \& Raichle, 1998) and DLPFC (Devrets \& Raichle, 1998), which is also known to inhibit saccades (Ettinger et al., 2007; Pierrot-Deseilligny et al., 2002; Ploner et al., 2005). Cortical regions important for holding visuospatial attention in the periphery and for covert shifts of attention were activated as well such as the precentral gyrus, the posterior parietal cortex (inferior parietal lobule and inferior parietal sulcus) and medial frontal gyrus (Kelley et al., 2008; Hopfinger et al., 2000; Corbetta \& Shulman, 2002; Beauchamp et al., 2001; Grosbras et al., 2005). The right-lateralized activation of the temporo-parietal junction is in line with previous studies suggesting an important role of this region in redirecting attention to task-relevant stimuli (Mitchell, 2008; Corbetta \& Shulman, 2002; Serences et al., 2005). Areas implicated in emotional processing including the insula, putamen and midbrain also showed enhanced activity (Lane et al., 1997; Davidson \& Irwin, 1999; Murphy et al., 2003; Phan et al., 2002; Wager et al., 2003). Face processing areas like the fusiform gyrus and STS exhibited increased activity (Haxby et al., 2000; 2002; Kanwisher et al., 1997). Furthermore, enhanced activity was observed in the middle temporal area (MT+/V5), which is known to be involved in the sensory analysis of stimuli (Corbetta et al., 2000; Corbetta \& Shulman, 2002) and in 
processing motion (Freitag et al., 1998; de Jong et al., 1994; Tootell et al., 1995a,b; Rees et al., 2000; Huk \& Heeger, 2002; Huk et al. 2002). Task-induced activity decreases were observed in a set of cortical regions found to be deactivated during attentionally demanding visuospatial tasks like the rACC (Devrets \& Raichle, 1998) and during goaldirected, cognitive activation conditions compared to low-level baselines like the medial prefrontal cortex, the posterior cingulate cortex, angular gyrus, and the superior frontal gyrus, which strongly overlaps with the "resting state" network (Raichle \& Snyder, 2007; Raichle et al., 2001; Gusnard \& Raichle, 2001; Shulman et al., 1997; Binder et al., 1999; Mazoyer et al., 2001).

\subsection{Conclusion}

This fMRI study provides further evidence for the notion that activity in the human amygdala strongly depends on attentional resources available, whereas the spatial location of emotional stimuli do not significantly affect the neuronal activity in the amygdala. Attentionally demanding cognitive tasks, which effectively consume processing resources, are associated with an active suppression of the amygdala response during these conditions relating to a default brain mode, which is suspended during many types of cognitive processing (Shulman et al., 1997; Raichle et al., 2001). Thus, the decreased activity during the active tasks may reflect the absence of processes that normally would occur during a passive condition. This implies that the preferential activation of the amygdala by emotional facial expressions may not only depend on attention to the faces, but instead may require that no suppression processes are active at the time of stimulation. In summary, our data suggest that the processing of emotional information is governed by top-down processes involved in selective attention. 


\section{Conclusion \& Outlook}

„In der Wissenschaft gleichen wir alle nur den Kindern, die am Rande des Wissens hie und da einen Kiesel aufheben, während sich der weite Ozean des Unbekannten vor unseren Augen erstreckt.“

Sir Isaac Newton

Every second our cognitive system is confronted with complex visual scenes that contain a variety of different objects. As a result of the limited processing capacity of the visual system, they cannot be fully processed at once. Therefore, attentional mechanisms determine the selection of information in favour of behaviourally relevant stimuli for enhanced processing, thereby providing a mechanism for the filtering of irrelevant and distracting information. Attention can be directed to specific parts of the visual field, where information processing is facilitated in contrast to unattended locations. The study of such visual spatial attention has been dominated by 'spotlight theories', claiming that selective attention can be compared to a spotlight illuminating anything located within the region covered by its beam. Several theories have been proposed using the idea of a spotlight as a metaphor to account for the distribution of attentional resources in visual space: 'single spotlight', 'zoom lens', and 'multiple spotlights' models. Recent studies have provided support for an ability to split this attentional focus to selectively process spatially separate locations.

In the first study I readdressed the issue of dividing attention among separated regions in the visual field. Using fMRI the signature for the presence of multiple spotlights of attention is the presence of multiple retinotopically specific foci of activation in striate and extrastriate visual areas. This approach was used to investigate the presence of such separable activations as a function of the eccentricity of the spatial foci of attention. Subjects had to deploy their attention either to a single peripheral location or two noncontiguous regions performing a match-mismatch judgement. 
First, the main aim of this study was the verification of the multiple spotlights theory as only one study so far investigated that issue using fMRI. As the split of the attentional focus among multiple regions in the visual field could be demonstrated, I sought to extend these findings to more peripheral parts of the visual field. The results showed that dividing attention lead to multiple spotlights of attention for central as well as more peripheral locations of the visual field. The multiple spotlights hypothesis was supported by the majority of the subjects, though the activation maps yielded not only increased activity restricted to the attended locations but also in intermediate regions. In order to account for these findings, I introduced a model considering the separability of the attentional spotlights. At a given threshold of the fMRI analysis, the neuronal response to multiple attended locations depends on the size of the spotlights of attention. A broad distribution of neuronal responsitivity corresponding to the attended locations creates a substantial overlap of two adjacent spotlights of attention and consequently results in additional attentional modulation in intermediate regions. The larger the width of the two spotlights of attention is the smaller is the separability of the two peaks of activity and the bigger is the overlap. Even a single region of activation can be explained by this model.

In summary, the results of this study demonstrated the human ability to split the attentional spotlight in order to deal effectively with information at different non-adjacent locations, resulting in retinotopically specific double-peaked attentional profiles in striate and extrastriate visual areas. The obtained increased activity in intermediate regions indicates an overlap of attentional spotlights that are wider than the behaviourally relevant locations necessitate.

This observed ability to divide attention among different spatial locations is known to be an attentionally demanding process, which depends on the availability of attentional resources and thus induces an increased attentional load. In my second fMRI study, I used the different task conditions of the first study to investigate the concept of high and low attentional load in relation to the processing of emotion-laden stimuli.

As outlined above, selective attention devotes cognitive resources to behavioural relevant stimuli and events thereby enhancing the processing of attended relative to unattended information. A primary way to determine the importance of a stimulus or event is to evaluate the emotional significance. Emotionally significant stimuli receive enhanced processing either preattentively ('automatically') or are given priority in the competition for selective attention. The amygdala has been identified to play a crucial role in the processing of emotional stimuli. It provides a coarse, rapid and automatic perception of 
emotional stimuli based on a subcortical route via the superior colliculus and the pulvinar nucleus of the thalamus, whereas a fine-grained perceptual representation of emotion-laden stimuli involves the occipital and temporal visual neocortex. Recent neuroimaging studies have produced conflicting results regarding the issue whether the processing of emotionladen stimuli is dependent upon the availability of attentional resources or entirely capacity free. Two main factors have been proposed to be responsible for the discrepancies: the differences in the perceptual attentional demands of the tasks used to divert attentional resources from emotional stimuli and the spatial location of the affective stimuli in the visual field.

No fMRI study to date investigated the impact of attentional load and spatial position of affective stimuli on face processing. My study provides a first attempt to resolve the discrepancies of previous studies relating to the issue whether the response in the amygdala is resource-dependent or not. One strong point of the study is that the different stimulus locations were tested within the same group of participants, which allowed a direct comparison between them. I investigated the effects of variable attentional load as well as different stimulus locations on the processing of emotional faces in the amygdala using fMRI. Participants had to either direct their attention to visual streams performing tasks of high and low attentional load or to faces matching for gender or expression.

The results revealed a strong attenuation of amygdala activity with higher attentional load. The spatial location of the emotional stimuli did not affect the neuronal response in the amygdala. These findings lend support to the concept that the processing of emotional stimuli is modulated by selective attention as the inhibition of amygdala response during attentionally demanding tasks reflects the dependence upon processing resources.

The complete suppression of amygdala response during all experimental conditions and especially during attend-faces trials was one striking finding as previous studies mainly demonstrated an increase in amygdala response to emotion-laden stimuli. For this reason control experiments were performed which involved a passive viewing condition indicating an increased amygdala response whereas the implication of a simple gender discrimination task induced a decreased activity in the amygdala. Furthermore, the spatial position showed to have an impact on the amygdala activity during the passive viewing condition. While an enhanced activity was observed during the processing of faces presented in the fovea and in the middle position, this response in the amygdala was extinguished when the emotional stimuli were displayed at the most eccentric positions. This finding is in direct contradiction to the idea that the amygdala is especially sensitive 
to behaviourally relevant stimuli in the periphery. The spatial frequency seems to play an important role in this context and might provide an explanation for the lack of amygdala responses to faces in the periphery. The amygdala has been found to be more activated in response to low spatial frequency filtered images in contrast to high spatial frequency ones presented in the fovea. Such a preference to low spatial frequency information in the amygdala is supported by the indirect connection via the superior colliculus and pulvinar to the magnocellular pathway which is known to be sensitive to low spatial frequencies and peripheral stimuli. In my next project, I am dealing with the issue concerning the relationship between eccentricity and spatial frequency. Future experiments are needed to further extend previous findings and investigate if the amygdala also preferentially responds to low spatial frequency stimuli in contrast to high spatial frequency ones presented in the periphery.

In conclusion, this thesis provides evidence for the split of attention among non-contiguous regions in the visual field in demonstrating separable peaks of activation in striate and extrastriate visual cortex. This split of attention produces a certain amount of attentional load that interacts with face processing. It has clearly been shown that the response of the amygdala to emotional stimuli is greatly modulated by attention. However, future studies need to address the issue of spatial location of the emotion-laden stimuli in the visual field to characterize the specific role of the amygdala within the face-processing system in more detail. 


\section{References}

Adolphs, R. (2002a). Neural systems for recognizing emotion. Curr Opin Neurobiol, 12(2), 169-177.

Adolphs, R. (2002b). Recognizing emotion from facial expressions: psychological and neurological mechanisms. Behav Cogn Neurosci Rev, 1(1), 21-62.

Adolphs, R., Damasio, H., Tranel, D., Cooper, G., \& Damasio, A. R. (2000). A role for somatosensory cortices in the visual recognition of emotion as revealed by threedimensional lesion mapping. J Neurosci, 20(7), 2683-2690.

Adolphs, R., Damasio, H., Tranel, D., \& Damasio, A. R. (1996). Cortical systems for the recognition of emotion in facial expressions. J Neurosci, 16(23), 7678-7687.

Adolphs, R., Gosselin, F., Buchanan, T. W., Tranel, D., Schyns, P., \& Damasio, A. R. (2005). A mechanism for impaired fear recognition after amygdala damage. Nature, 433(7021), 68-72.

Adolphs, R., Schul, R., \& Tranel, D. (1998a). Intact recognition of facial emotion in Parkinson's disease. Neuropsychology, 12(2), 253-258.

Adolphs, R., Tranel, D., \& Damasio, A. R. (1998b). The human amygdala in social judgment. Nature, 393(6684), 470-474.

Adolphs, R., Tranel, D., \& Damasio, A. R. (2003). Dissociable neural systems for recognizing emotions. Brain Cogn, 52(1), 61-69.

Adolphs, R., Tranel, D., Damasio, H., \& Damasio, A. (1994). Impaired recognition of emotion in facial expressions following bilateral damage to the human amygdala. Nature, 372(6507), 669-672.

Adolphs, R., Tranel, D., Damasio, H., \& Damasio, A. R. (1995). Fear and the human amygdala. J Neurosci, 15(9), 5879-5891.

Adolphs, R., Tranel, D., Hamann, S., Young, A. W., Calder, A. J., Phelps, E. A., et al. (1999). Recognition of facial emotion in nine individuals with bilateral amygdala damage. Neuropsychologia, 37(10), 1111-1117.

Aggleton, J. P. (1993). The contribution of the amygdala to normal and abnormal emotional states. Trends Neurosci, 16(8), 328-333.

Aggleton, J. P., \& Brown, M. W. (1999). Episodic memory, amnesia, and the hippocampal-anterior thalamic axis. Behav Brain Sci, 22(3), 425-444; discussion 444-489. 
Aguirre, G. K., Zarahn, E., \& D'Esposito, M. (1998). The variability of human, BOLD hemodynamic responses. Neuroimage, 8(4), 360-369.

Allison, T., Puce, A., \& McCarthy, G. (2000). Social perception from visual cues: role of the STS region. Trends Cogn Sci, 4(7), 267-278.

Amaral, D. G., Behniea, H., \& Kelly, J. L. (2003). Topographic organization of projections from the amygdala to the visual cortex in the macaque monkey. Neuroscience, 118(4), 1099-1120.

Amaral, D. G., Price, J. L., Pitkanen, A., \& Carmichael, S. T. (1992). Anatomical organization of the primate amagdaloid complex. In J. P. Aggleton (Ed.), The amygdala: Neurobiological aspects of emotion, memory and mental dysfunction (pp. 1-66). New York: Wiley.

Anderson, A. K., Christoff, K., Panitz, D., De Rosa, E., \& Gabrieli, J. D. (2003). Neural correlates of the automatic processing of threat facial signals. J Neurosci, 23(13), $5627-5633$.

Anderson, A. K., \& Phelps, E. A. (2001). Lesions of the human amygdala impair enhanced perception of emotionally salient events. Nature, 411(6835), 305-309.

Anderson, A. K., Spencer, D. D., Fulbright, R. K., \& Phelps, E. A. (2000). Contribution of the anteromedial temporal lobes to the evaluation of facial emotion. Neuropsychology, 14(4), 526-536.

Arthurs, O. J., \& Boniface, S. (2002). How well do we understand the neural origins of the fMRI BOLD signal? Trends Neurosci, 25(1), 27-31.

Awh, E., \& Pashler, H. (2000). Evidence for split attentional foci. J Exp Psychol Hum Percept Perform, 26(2), 834-846.

Bandettini, P. A., Wong, E. C., Hinks, R. S., Tikofsky, R. S., \& Hyde, J. S. (1992). Time course EPI of human brain function during task activation. Magn Reson Med, 25(2), 390-397.

Banich, M. T., Milham, M. P., Atchley, R. A., Cohen, N. J., Webb, A., Wszalek, T., et al. (2000). Prefrontal regions play a predominant role in imposing an attentional 'set': evidence from fMRI. Brain Res Cogn Brain Res, 10(1-2), 1-9.

Barbas, H. (2000). Connections underlying the synthesis of cognition, memory, and emotion in primate prefrontal cortices. Brain Res Bull, 52(5), 319-330.

Barbas, H., \& Pandya, D. N. (1989). Architecture and intrinsic connections of the prefrontal cortex in the rhesus monkey. J Comp Neurol, 286(3), 353-375. 
Barbas, H., Saha, S., Rempel-Clower, N., \& Ghashghaei, T. (2003). Serial pathways from primate prefrontal cortex to autonomic areas may influence emotional expression. BMC Neurosci, 4, 25.

Bear, M. F., Connors, B. W., \& Paradiso, M. A. (2007). Neuroscience (Third ed.). Baltimore: Lippincott Williams \& Wilkins.

Beauchamp, M. S., Cox, R. W., \& DeYoe, E. A. (1997). Graded effects of spatial and featural attention on human area MT and associated motion processing areas. $J$ Neurophysiol, 78(1), 516-520.

Bechara, A., Damasio, A. R., Damasio, H., \& Anderson, S. W. (1994). Insensitivity to future consequences following damage to human prefrontal cortex. Cognition, 50(1-3), 7-15.

Bechara, A., Damasio, H., Tranel, D., \& Anderson, S. W. (1998). Dissociation Of working memory from decision making within the human prefrontal cortex. $J$ Neurosci, $18(1), 428-437$.

Bechara, A., Tranel, D., Damasio, H., Adolphs, R., Rockland, C., \& Damasio, A. R. (1995). Double dissociation of conditioning and declarative knowledge relative to the amygdala and hippocampus in humans. Science, 269(5227), 1115-1118.

Bechara, A., Tranel, D., Damasio, H., \& Damasio, A. R. (1996). Failure to respond autonomically to anticipated future outcomes following damage to prefrontal cortex. Cereb Cortex, 6(2), 215-225.

Beck, J., \& Ambler, B. (1973). The effect of concentrated and distributed attention on peripheral acuity. Percept Psychophys, 14, 225-230.

Berson, D. M. (1988). Retinal and cortical inputs to cat superior colliculus: composition, convergence and laminar specificity. Prog Brain Res, 75, 17-26.

Berson, D. M., \& Stein, J. J. (1995). Retinotopic organization of the superior colliculus in relation to the retinal distribution of afferent ganglion cells. Vis Neurosci, 12(4), 671-686.

Bichot, N. P., Cave, K. R., \& Pashler, H. (1999). Visual selection mediated by location: feature-based selection of noncontiguous locations. Percept Psychophys, 61(3), 403-423.

Binder, J. R., Frost, J. A., Hammeke, T. A., Bellgowan, P. S., Rao, S. M., \& Cox, R. W. (1999). Conceptual processing during the conscious resting state. A functional MRI study. J Cogn Neurosci, 11(1), 80-95. 
Bishop, S., Duncan, J., Brett, M., \& Lawrence, A. D. (2004). Prefrontal cortical function and anxiety: Controlling attention to threat-related stimuli. Nat Neurosci, 7, 184188.

Bishop, S. J., Jenkins, R., \& Lawrence, A. D. (2007). Neural processing of fearful faces: effects of anxiety are gated by perceptual capacity limitations. Cerebral Cortex, 17, 1595-1603.

Blair, H. T., Sotres-Bayon, F., Moita, M. A., \& Ledoux, J. E. (2005). The lateral amygdala processes the value of conditioned and unconditioned aversive stimuli. Neuroscience, 133(2), 561-569.

Blair, R. J., Morris, J. S., Frith, C. D., Perrett, D. I., \& Dolan, R. J. (1999). Dissociable neural responses to facial expressions of sadness and anger. Brain, 122 ( Pt 5), 883893.

Bloch, F. (1946). Nuclear induction. Physiology Review, 70, 460-474.

Blonder, L. X., Gur, R. E., \& Gur, R. C. (1989). The effects of right and left hemiparkinsonism on prosody. Brain and Language, 36, 193-207.

Bonda, E., Petrides, M., Ostry, D., \& Evans, A. (1996). Specific involvement of human parietal systems and the amygdala in the perception of biological motion. $J$ Neurosci, 16(11), 3737-3744.

Bonvento, G., Sibson, N., \& Pellerin, L. (2002). Does glutamate image your thoughts? Trends Neurosci, 25(7), 359-364.

Botvinick, M. M., Cohen, J. D., \& Carter, C. S. (2004). Conflict monitoring and anterior cingulate cortex: an update. Trends Cogn Sci, 8(12), 539-546.

Bradley, M. M., \& Lang, P. J. (1994). Measuring emotion: the Self-Assessment Manikin and the Semantic Differential. J Behav Ther Exp Psychiatry, 25(1), 49-59.

Brefczynski, J. A., \& DeYoe, E. A. (1999). A physiological correlate of the 'spotlight' of visual attention. Nat Neurosci, 2(4), 370-374.

Breiter, H. C., Etcoff, N. L., Whalen, P. J., Kennedy, W. A., Rauch, S. L., Buckner, R. L., et al. (1996a). Response and habituation of the human amygdala during visual processing of facial expression. Neuron, 17(5), 875-887.

Breiter, H. C., Gollub, R. L., Weisskoff, R. M., Kennedy, D. N., Makris, N., Berke, J. D., et al. (1997). Acute effects of cocaine on human brain activity and emotion. Neuron, 19(3), 591-611. 
Breiter, H. C., Rauch, S. L., Kwong, K. K., Baker, J. R., Weisskoff, R. M., Kennedy, D. N., et al. (1996b). Functional magnetic resonance imaging of symptom provocation in obsessive-compulsive disorder. Arch Gen Psychiatry, 53(7), 595-606.

Broadbent, D. E. (1958). Perception and Communication. New York: Pergamon Press.

Broadbent, D. E. (1982). Task combination and selective intake of information. Acta Psychol (Amst), 50(3), 253-290.

Broks, P., Young, A. W., Maratos, E. J., Coffey, P. J., Calder, A. J., Isaac, C. L., et al. (1998). Face processing impairments after encephalitis: amygdala damage and recognition of fear. Neuropsychologia, 36(1), 59-70.

Bruce, V., \& Young, A. (1986). Understanding face recognition. Br J Psychol, 77 ( Pt 3), 305-327.

Buccino, G., Binkofski, F., Fink, G. R., Fadiga, L., Fogassi, L., Gallese, V., et al. (2001). Action observation activates premotor and parietal areas in a somatotopic manner: an fMRI study. Eur J Neurosci, 13(2), 400-404.

Bullier, J. (2001). Integrated model of visual processing. Brain Res Brain Res Rev, 36(2-3), 96-107.

Bush, G., Luu, P., \& Posner, M. I. (2000). Cognitive and emotional influences in anterior cingulate cortex. Trends Cogn Sci, 4(6), 215-222.

Bushnell, M. C., Goldberg, M. E., \& Robinson, D. L. (1981). Behavioral enhancement of visual responses in monkey cerebral cortex. I. Modulation in posterior parietal cortex related to selective visual attention. J Neurophysiol, 46(4), 755-772.

Bushong, S. C. (2003). Magnetic Resonance Imaging: Physical and Biological Principles (Third ed.). Houston, Texas: Mosby.

Cahill, L., \& McGaugh, J. L. (1998). Mechanisms of emotional arousal and lasting declarative memory. Trends Neurosci, 21(7), 294-299.

Calder, A. J., Beaver, J. D., Winston, J. S., Dolan, R. J., Jenkins, R., Eger, E., et al. (2007). Separate coding of different gaze directions in the superior temporal sulcus and inferior parietal lobule. Curr Biol, 17(1), 20-25.

Calder, A. J., Keane, J., Manes, F., Antoun, N., \& Young, A. W. (2000). Impaired recognition and experience of disgust following brain injury. Nat Neurosci, 3(11), 1077-1078.

Calder, A. J., Lawrence, A. D., \& Young, A. W. (2001). Neuropsychology of fear and loathing. Nat Rev Neurosci, 2(5), 352-363. 
Calder, A. J., Young, A. W., Keane, J., \& Dean, M. (2000). Configural information in facial expression perception. J Exp Psychol Hum Percept Perform, 26(2), 527-551.

Calder, A. J., Young, A. W., Rowland, D., Perrett, D. I., Hodges, J. R., \& Etcoff, N. L. (1996). Facial Emotion Recognition after Bilateral Amygdala Damage: Differentially Severe Impairment of Fear. Cognitive Neuropsychology, 13(5), 699745.

Callejas, A., Lupianez, J., Funes, M. J., \& Tudela, P. (2005). Modulations among the alerting, orienting and executive control networks. Exp Brain Res, 167(1), 27-37.

Callejas, A., Lupianez, J., \& Tudela, P. (2004). The three attentional networks: on their independence and interactions. Brain Cogn, 54(3), 225-227.

Cameron, E. L., Tai, J. C., \& Carrasco, M. (2002). Covert attention affects the psychometric function of contrast sensitivity. Vision Res, 42(8), 949-967.

Carter, C. S., Braver, T. S., Barch, D. M., Botvinick, M. M., Noll, D., \& Cohen, J. D. (1998). Anterior cingulate cortex, error detection, and the online monitoring of performance. Science, 280(5364), 747-749.

Castiello, U., \& Umilta, C. (1990). Size of the attentional focus and efficiency of processing. Acta Psychol (Amst), 73(3), 195-209.

Castiello, U., \& Umilta, C. (1992). Splitting focal attention. J Exp Psychol Hum Percept Perform, 18(3), 837-848.

Chao, L. L., Haxby, J. V., \& Martin, A. (1999a). Attribute-based neural substrates in temporal cortex for perceiving and knowing about objects. Nat Neurosci, 2(10), 913-919.

Chao, L. L., Martin, A., \& Haxby, J. V. (1999b). Are face-responsive regions selective only for faces? Neuroreport, 10(14), 2945-2950.

Chastain, G. (1992a). Analog versus discrete shifts of attention across the visual field. Psychol Res, 54(3), 175-181.

Chastain, G. (1992b). Time-course of sensitivity changes as attention shifts to an unpredictable location. J Gen Psychol, 119(2), 105-111.

Chawla, D., Rees, G., \& Friston, K. J. (1999). The physiological basis of attentional modulation in extrastriate visual areas. Nat Neurosci, 2(7), 671-676.

Cherry, E. C. (1953). Some experiments on the recognition of speech, with one and two ears. Journal of the Acoustical Society of America, 25, 975-979.

Compton, R. J. (2003). The interface between emotion and attention: a review of evidence from psychology and neuroscience. Behav Cogn Neurosci Rev, 2(2), 115-129. 
Connor, C. E., Preddie, D. C., Gallant, J. L., \& Van Essen, D. C. (1997). Spatial attention effects in macaque area V4. J Neurosci, 17(9), 3201-3214.

Corbetta, M., Kincade, J. M., Ollinger, J. M., McAvoy, M. P., \& Shulman, G. L. (2000). Voluntary orienting is dissociated from target detection in human posterior parietal cortex. Nat Neurosci, 3(3), 292-297.

Corbetta, M., Miezin, F. M., Dobmeyer, S., Shulman, G. L., \& Petersen, S. E. (1990). Attentional modulation of neural processing of shape, color, and velocity in humans. Science, 248(4962), 1556-1559.

Corbetta, M., Miezin, F. M., Dobmeyer, S., Shulman, G. L., \& Petersen, S. E. (1991). Selective and divided attention during visual discriminations of shape, color, and speed: functional anatomy by positron emission tomography. J Neurosci, 11(8), 2383-2402.

Corbetta, M., Miezin, F. M., Shulman, G. L., \& Petersen, S. E. (1993). A PET study of visuospatial attention. J Neurosci, 13(3), 1202-1226.

Corbetta, M., \& Shulman, G. L. (2002). Control of goal-directed and stimulus-driven attention in the brain. Nat Rev Neurosci, 3(3), 201-215.

Costen, N. P., Parker, D. M., \& Craw, I. (1996). Effects of high-pass and low-pass spatial filtering on face identification. Percept Psychophys, 58(4), 602-612.

Crick, F. (1984). Function of the thalamic reticular complex: the searchlight hypothesis. Proc Natl Acad Sci U S A, 81(14), 4586-4590.

Critchley, H., Daly, E., Phillips, M., Brammer, M., Bullmore, E., Williams, S., et al. (2000). Explicit and implicit neural mechanisms for processing of social information from facial expressions: a functional magnetic resonance imaging study. Hum Brain Mapp, 9(2), 93-105.

Critchley, H. D., Mathias, C. J., Josephs, O., O'Doherty, J., Zanini, S., Dewar, B. K., et al. (2003). Human cingulate cortex and autonomic control: converging neuroimaging and clinical evidence. Brain, 126(Pt 10), 2139-2152.

Critchley, H. D., Tang, J., Glaser, D., Butterworth, B., \& Dolan, R. J. (2005). Anterior cingulate activity during error and autonomic response. Neuroimage, 27(4), 885895.

Curtis, C. E., \& D'Esposito, M. (2003). Persistent activity in the prefrontal cortex during working memory. Trends Cogn Sci, 7(9), 415-423.

Damasio, A. (1995). Descartes Error. London: Picador. 
Damasio, A. R., Grabowski, T. J., Bechara, A., Damasio, H., Ponto, L. L., Parvizi, J., et al. (2000). Subcortical and cortical brain activity during the feeling of self-generated emotions. Nat Neurosci, 3(10), 1049-1056.

Darwin, C. (1965). The Expression of the Emotions in Man and Animals. Chicago: University of Chicago Press.

Das, P., Kemp, A. H., Liddell, B. J., Brown, K. J., Olivieri, G., Peduto, A., et al. (2005). Pathways for fear perception: modulation of amygdala activity by thalamo-cortical systems. Neuroimage, 26(1), 141-148.

Davidson, R. J. (1992). Anterior cerebral asymmetry and the nature of emotion. Brain Cogn, 20(1), 125-151.

Davidson, R. J. (1998). Affective style and affective disorders: perspectives from affective neuroscience. Cognition Emotion, 12, 307-320.

Davidson, R. J., Ekman, P., Saron, C. D., Senulis, J. A., \& Friesen, W. V. (1990). Approach-withdrawal and cerebral asymmetry: emotional expression and brain physiology. I. J Pers Soc Psychol, 58(2), 330-341.

Davidson, R. J., \& Irwin, W. (1999). The functional neuroanatomy of emotion and affective style. Trends Cogn Sci, 3(1), 11-21.

Davis, K. D., Taylor, K. S., Hutchison, W. D., Dostrovsky, J. O., McAndrews, M. P., Richter, E. O., et al. (2005). Human anterior cingulate cortex neurons encode cognitive and emotional demands. J Neurosci, 25(37), 8402-8406.

Davis, M., \& Whalen, P. J. (2001). The amygdala: vigilance and emotion. Mol Psychiatry, 6(1), 13-34.

De Gelder, B., Vroomen, J., Pourtois, G., \& Weiskrantz, L. (1999). Non-conscious recognition of affect in the absence of striate cortex. Neuroreport, 10, 3759-3763.

de Jong, B. M., Shipp, S., Skidmore, B., Frackowiak, R. S., \& Zeki, S. (1994). The cerebral activity related to the visual perception of forward motion in depth. Brain, 117 ( Pt 5), 1039-1054.

Decety, J., \& Grezes, J. (1999). Neural mechanisms subserving the perception of human actions. Trends Cogn Sci, 3(5), 172-178.

Derryberry, D., \& Reed, M. A. (2002). Anxiety-related attentional biases and their regulation by attentional control. J Abnorm Psychol, 111(2), 225-236.

Desimone, R., \& Duncan, J. (1995). Neural mechanisms of selective visual attention. Annu Rev Neurosci, 18, 193-222. 
Deutsch, J. A., \& Deutsch, D. (1963). Attention: some theoretical considerations. Psychological Review, 70, 80-90.

Devinsky, O., Morrell, M. J., \& Vogt, B. A. (1995). Contributions of anterior cingulate cortex to behaviour. Brain, 118 ( Pt 1), 279-306.

DeYoe, E. A., Carman, G. J., Bandettini, P., Glickman, S., Wieser, J., Cox, R., et al. (1996). Mapping striate and extrastriate visual areas in human cerebral cortex. Proc Natl Acad Sci U S A, 93(6), 2382-2386.

DeYoe, E. A., \& Van Essen, D. C. (1988). Concurrent processing streams in monkey visual cortex. Trends Neurosci., 11, 219-226.

Doallo, S., Cadaveira, F., \& Rodriguez Holguin, S. (2007). Time course of attentional modulations on automatic emotional processing. Neurosci Lett, 418(1), 111-116.

Doallo, S., Holguin, S. R., \& Cadaveira, F. (2006). Attentional load affects automatic emotional processing: evidence from event-related potentials. Neuroreport, 17(17), 1797-1801.

Dolan, R. J. (2003). Emotion, cognition, and behavior. Science, 298, 1191-1194.

Downing, C. J. (1988). Expectancy and visual-spatial attention: effects on perceptual quality. J Exp Psychol Hum Percept Perform, 14(2), 188-202.

Drevets, W. C., \& Raichle, M. E. (1995). PET imaging studies of human emotional disorders. In M. S. Gazzaniga (Ed.), The cognitive neurosciences. Cambridge, MA: MIT Press.

Drevets, W. C., \& Raichle, M. E. (1998). Reciprocal suppression of regional cerebral blood flow during emotional versus higher cognitive processes: implications for interactions between emotion and cognition. Cognition and Emotion, 12(3), 353385.

Driver, J. (2001). A selective review of selective attention research from the past century. Br J Psychol, 92 Part 1, 53-78.

Driver, J., \& Baylis, G. C. (1989). Movement and visual attention: the spotlight metaphor breaks down. J Exp Psychol Hum Percept Perform, 15(3), 448-456.

Duncan, J. (1984). Selective attention and the organization of visual information. J Exp Psychol Gen, 113(4), 501-517.

Duncan, J. (1996). Cooperating brain systems in selective perception and action. In T. Inui, McClelland, J.L. (Ed.), Attention and Performance (Vol. Vol XVI). Cambridge MA: MIT Press. 
Duncan, J., Seitz, R. J., Kolodny, J., Bor, D., Herzog, H., Ahmed, A., et al. (2000). A neural basis for general intelligence. Science, 289(5478), 457-460.

Duncan, J., Ward, R., \& Shapiro, K. (1994). Direct measurement of attentional dwell time in human vision. Nature, 369(6478), 313-315.

Eason, R., Harter, M., \& White, C. (1969). Effects of attention and arousal on visually evoked cortical potentials and reaction time in man. Physiology and Behavior, 4, 283-289.

Eger, E., Jedynak, A., Iwaki, T., \& Skrandies, W. (2003). Rapid extraction of emotional expression: evidence from evoked potential fields during brief presentation of face stimuli. Neuropsychologia, 41(7), 808-817.

Egeth, H. (1977). Attention and preattention. In G. H. Bower (Ed.), The Psychology of Learning and Motivation (pp. 277-320).

Eimer, M. (2000). An ERP study of sustained spatial attention to stimulus eccentricity. Biol Psychol, 52(3), 205-220.

Eimer, M., \& Holmes, A. (2002). An ERP study on the time course of emotional face processing. Neuroreport, 13(4), 427-431.

Eimer, M., Holmes, A., \& McGlone, F. P. (2003). The role of spatial attention in the processing of facial expression: an ERP study of rapid brain responses to six basic emotions. Cogn Affect Behav Neurosci, 3(2), 97-110.

Ekman, P. (1982). Emotion in the Human Face. Cambridge, England: Cambridge University Press.

Ekman, P., \& Friesen, W. V. (1971). Constants across cultures in the face and emotion. $J$. Pers. Soc. Psychol., 17, 124-129.

Eltiti, S., Wallace, D., \& Fox, E. (2005). Selective target processing: perceptual load or distractor salience? Percept Psychophys, 67(5), 876-885.

Emery, N. J., \& Amaral, D. G. (1999). The role of the amygdala in primate social cognition. In R. D. Lane \& L. Nadel (Eds.), Cognitive neuroscience of emotion (pp. 156-191). Oxford, UK: Oxford University Press.

Engell, A. D., \& Haxby, J. V. (2007). Facial expression and gaze-direction in human superior temporal sulcus. Neuropsychologia, 45(14), 3234-3241.

Eriksen, B. A., \& Eriksen, C. W. (1974). Effects of noise letters upon the identification of a target letter in a nonsearch task. Perception \& Psychophysics, 16, 143-149.

Eriksen, C. W., \& Hoffman, J. E. (1972). Temporal and spatial characteristics of selective encoding from visual displays. Perception and Psychophysics, 12, 201-204. 
Eriksen, C. W., \& Hoffman, J. E. (1973). The extent of processing of noise elements during selective encoding from visual display. Percept Psychophys, 14, 155-160.

Eriksen, C. W., \& Murphy, T. D. (1987). Movement of attentional focus across the visual field: a critical look at the evidence. Percept Psychophys, 42(3), 299-305.

Eriksen, C. W., \& St. James, J. D. (1986). Visual attention within and around the field of focal attention: A zoom lens model. Perception and Psychophysics, 40, 225-240.

Eriksen, C. W., \& Webb, J. M. (1989). Shifting of attentional focus within and about a visual display. Percept Psychophys, 45(2), 175-183.

Eriksen, C. W., \& Yeh, Y. Y. (1985). Allocation of attention in the visual field. J Exp Psychol Hum Percept Perform, 11(5), 583-597.

Erthal, F. S., de Oliveira, L., Mocaiber, I., Pereira, M. G., Machado-Pinheiro, W., Volchan, E., et al. (2005). Load-dependent modulation of affective picture processing. Cogn Affect Behav Neurosci, 5(4), 388-395.

Esteves, F., \& Ohman, A. (1993). Masking the face: recognition of emotional facial expressions as a function of the parameters of backward masking. Scand J Psychol, 34(1), 1-18.

Ettinger, U., Ffytche, D. H., Kumari, V., Kathmann, N., Reuter, B., Zelaya, F., et al. (2007). Decomposing the Neural Correlates of Antisaccade Eye Movements Using Event-Related fMRI. Cereb Cortex.

Fan, J., McCandliss, B. D., Fossella, J., Flombaum, J. I., \& Posner, M. I. (2005). The activation of attentional networks. Neuroimage, 26(2), 471-479.

Fan, J., McCandliss, B. D., Sommer, T., Raz, A., \& Posner, M. I. (2002). Testing the efficiency and independence of attentional networks. J Cogn Neurosci, 14(3), 340347.

Felleman, D. J., \& Van Essen, D. C. (1991). Distributed hierarchical processing in the primate cerebral cortex. Cereb. Cortex, 1, 1-47.

Fichtenholtz, H. M., Dean, H. L., Dillon, D. G., Yamasaki, H., McCarthy, G., \& LaBar, K. S. (2004). Emotion-attention network interactions during a visual oddball task. Brain Res Cogn Brain Res, 20(1), 67-80.

Fiorentini, A., Maffei, L., \& Sandini, G. (1983). The role of high spatial frequencies in face perception. Perception, 12(2), 195-201.

Fischer, H., Wright, C. I., Whalen, P. J., McInerney, S. C., Shin, L. M., \& Rauch, S. L. (2003). Brain habituation during repeated exposure to fearful and neutral faces: a functional MRI study. Brain Res Bull, 59(5), 387-392. 
Fitzgerald, D. A., Angstadt, M., Jelsone, L. M., Nathan, P. J., \& Phan, K. L. (2006). Beyond threat: amygdala reactivity across multiple expressions of facial affect. Neuroimage, 30(4), 1441-1448.

Fox, P. T., \& Raichle, M. E. (1986). Focal physiological uncoupling of cerebral blood flow and oxydative metabolism during somatosensory stimulation in human subjects. Proceedings of National Academy of Sciences USA, 83(4), 1140-1144.

Fox, P. T., Raichle, M. E., Mintun, M. A., \& Dence, C. (1988). Nonoxidative glucose consumption during focal physiologic neural activity. Science, 241, 462-464.

Frahm, J., Bruhn, H., Merboldt, K. D., \& Hanicke, W. (1992). Dynamic MR imaging of human brain oxygenation during rest and photic stimulation. J Magn Reson Imaging, 2(5), 501-505.

Freeman, E., Sagi, D., \& Driver, J. (2001). Lateral interactions between targets and flankers in low-level vision depend on attention to the flankers. Nat Neurosci, 4(10), 1032-1036.

Freitag, P., Greenlee, M. W., Lacina, T., Scheffler, K., \& Radu, E. W. (1998). Effect of eye movements on the magnitude of functional magnetic resonance imaging responses in extrastriate cortex during visual motion perception. Exp Brain Res, 119(4), 409414.

Friston, K. J., Frith, C. D., \& Frackowiak, R. S. J. (1993a). Time-dependent changes in effective connectivity measured with PET. Hum. Brain Mapp., 1, 69-79.

Friston, K. J., Frith, C. D., Liddle, P. F., \& Frackowiak, R. S. (1993b). Functional connectivity: the principal-component analysis of large (PET) data sets. J Cereb Blood Flow Metab, 13(1), 5-14.

Frith, C. (2001). A framework for studying the neural basis of attention. Neuropsychologia, 39(12), 1367-1371.

Fuster, J. M., Bauer, R. H., \& Jervey, J. P. (1985). Functional interactions between inferotemporal and prefrontal cortex in a cognitive task. Brain Res, 330(2), 299307.

Gallese, V., Fadiga, L., Fogassi, L., \& Rizzolatti, G. (1996). Action recognition in the premotor cortex. Brain, 119 ( Pt 2), 593-609.

Gandhi, S. P., Heeger, D. J., \& Boynton, G. M. (1999). Spatial attention affects brain activity in human primary visual cortex. Proc Natl Acad Sci U S A, 96(6), 33143319. 
Gauthier, I., Skudlarski, P., Gore, J. C., \& Anderson, A. W. (2000). Expertise for cars and birds recruits brain areas involved in face recognition. Nat Neurosci, 3(2), 191-197.

Gauthier, I., Tarr, M. J., Anderson, A. W., Skudlarski, P., \& Gore, J. C. (1999). Activation of the middle fusiform 'face area' increases with expertise in recognizing novel objects. Nat Neurosci, 2(6), 568-573.

Genovese, C. R., Lazar, N. A., \& Nichols, T. (2002). Thresholding of statistical maps in functional neuroimaging using the false discovery rate. Neuroimage, 15(4), 870878.

George, M. S., Ketter, T. A., Parekh, P. I., Herscovitch, P., \& Post, R. M. (1996). Gender differences in regional cerebral blood flow during transient self-induced sadness or happiness. Biol Psychiatry, 40(9), 859-871.

Goodale, M. A., \& Milner, A. D. (1992). Separate visual pathways for perception and action. Trends Neurosci, 15(1), 20-25.

Gorno-Tempini, M. L., Pradelli, S., Serafini, M., Pagnoni, G., Baraldi, P., Porro, C., et al. (2001). Explicit and incidental facial expression processing: an fMRI study. Neuroimage, 14, 465-473.

Gray, J. M., Young, A. W., Barker, W. A., Curtis, A., \& Gibson, D. (1997). Impaired recognition of disgust in Huntington's disease gene carriers. Brain, 120 ( Pt 11), 2029-2038.

Gray, J. R., Braver, T. S., \& Raichle, M. E. (2002). Integration of emotion and cognition in the lateral prefrontal cortex. Proc Natl Acad Sci U S A, 99(6), 4115-4120.

Greenstein, B., \& Greenstein, A. (2000). Color Atlas of Neuroscience - Neuroanatomy and Neurophysiology. Stuttgart, New York: Thieme.

Griffiths, P. E. (1997). What Emotions Really Are. Chicago: University of Chicago Press.

Grill-Spector, K., Knouf, N., \& Kanwisher, N. (2004). The fusiform face area subserves face perception, not generic within-category identification. Nat Neurosci, 7(5), 555562.

Grill-Spector, K., \& Malach, R. (2004). The human visual cortex. Annu Rev Neurosci, 27, 649-677.

Grill-Spector, K., Sayres, R., \& Ress, D. (2006). High-resolution imaging reveals highly selective nonface clusters in the fusiform face area. Nat Neurosci, 9(9), 1177-1185.

Grosbras, M. H., Laird, A. R., \& Paus, T. (2005). Cortical regions involved in eye movements, shifts of attention, and gaze perception. Hum Brain Mapp, 25(1), 140154. 
Gur, R. C., Schroeder, L., Turner, T., McGarth, C., Chan, R. M., Turetsky, B. I., et al. (2002). Brain activation during facial emotion processing. Neuroimage, 16, 651662.

Gur, R. C., Skolnick, B. E., \& Gur, R. E. (1994). Effects of emotional discrimination tasks on cerebral blood flow: Regional activation and its relation to performance. Brain and Cognition, 25(2), 271-286.

Gusnard, D. A., Raichle, M. E., \& Raichle, M. E. (2001). Searching for a baseline: functional imaging and the resting human brain. Nat Rev Neurosci, 2(10), 685-694.

Habel, U., Klein, M., Kellermann, T., Shah, N. J., \& Schneider, F. (2005). Same or different? Neural correlates of happy and sad mood in healthy males. Neuroimage, 26(1), 206-214.

Habel, U., Windischberger, C., Derntl, B., Robinson, S., Kryspin-Exner, I., Gur, R. C., et al. (2007). Amygdala activation and facial expressions: explicit emotion discrimination versus implicit emotion processing. Neuropsychologia, 45(10), 2369-2377.

Hahn, S., \& Kramer, A. F. (1998). Further evidence of the division of attention among non-contiguous locations. Visual Cognition, 5, 217-256.

Halgren, E., Baudena, P., Heit, G., Clarke, J. M., Marinkovic, K., \& Clarke, M. (1994). Spatio-temporal stages in face and word processing. I. Depth-recorded potentials in the human occipital, temporal and parietal lobes [corrected]. J Physiol Paris, 88(1), $1-50$.

Halgren, E., Dale, A. M., Sereno, M. I., Tootell, R. B., Marinkovic, K., \& Rosen, B. R. (1999). Location of human face-selective cortex with respect to retinotopic areas. Hum Brain Mapp, 7(1), 29-37.

Halgren, E., Raij, T., Marinkovic, K., Jousmaki, V., \& Hari, R. (2000). Cognitive response profile of the human fusiform face area as determined by MEG. Cereb Cortex, 10(1), 69-81.

Hamann, S., \& Canli, T. (2004). Individual differences in emotion processing. Curr Opin Neurobiol, 14(2), 233-238.

Hari, R., Forss, N., Avikainen, S., Kirveskari, E., Salenius, S., \& Rizzolatti, G. (1998). Activation of human primary motor cortex during action observation: a neuromagnetic study. Proc Natl Acad Sci U S A, 95(25), 15061-15065. 
Hariri, A. R., Bookheimer, S. Y., \& Mazziotta, J. C. (2000). Modulating emotional responses: effects of a neocortical network on the limbic system. Neuroreport, 11(1), 43-48.

Hasselmo, M. E., Rolls, E. T., \& Baylis, G. C. (1989). The role of expression and identity in the face-selective responses of neurons in the temporal visual cortex of the monkey. Behav Brain Res, 32(3), 203-218.

Hasson, U., Harel, M., Levy, I., \& Malach, R. (2003). Large-scale mirror-symmetry organization of human occipito-temporal object areas. Neuron, 37(6), 1027-1041.

Hasson, U., Levy, I., Behrmann, M., Hendler, T., \& Malach, R. (2002). Eccentricity bias as an organizing principle for human high-order object areas. Neuron, 34(3), 479-490.

Haxby, J. V., Gobbini, M. I., Furey, M. L., Ishai, A., Schouten, J. L., \& Pietrini, P. (2001). Distributed and overlapping representations of faces and objects in ventral temporal cortex. Science, 293(5539), 2425-2430.

Haxby, J. V., Hoffman, E. A., \& Gobbini, M. I. (2000). The distributed human neural system for face perception. Trends Cogn Sci, 4(6), 223-233.

Haxby, J. V., Hoffman, E. A., \& Gobbini, M. I. (2002). Human neural systems for face recognition and social communication. Biol Psychiatry, 51(1), 59-67.

Haxby, J. V., Horwitz, B., Ungerleider, L. G., Maisog, J. M., Pietrini, P., \& Grady, C. L. (1994). The functional organization of human extrastriate cortex: a PET-rCBF study of selective attention to faces and locations. J Neurosci, 14(11 Pt 1), 63366353.

Haxby, J. V., Ungerleider, L. G., Clark, V. P., Schouten, J. L., Hoffman, E. A., \& Martin, A. (1999). The effect of face inversion on activity in human neural systems for face and object perception. Neuron, 22(1), 189-199.

Hayes, T., Morrone, M. C., \& Burr, D. C. (1986). Recognition of positive and negative bandpass-filtered images. Perception, 15(5), 595-602.

Haynes, J. D., Tregellas, J., \& Rees, G. (2005). Attentional integration between anatomically distinct stimulus representations in early visual cortex. Proc Natl Acad Sci U S A, 102(41), 14925-14930.

Heeger, D. J., \& Ress, D. (2002). What does fMRI tell us about neuronal activity? Nat Rev Neurosci, 3(2), 142-151.

Heinze, H. J., Luck, S. J., Munte, T. F., Gos, A., Mangun, G. R., \& Hillyard, S. A. (1994). Attention to adjacent and separate positions in space: an electrophysiological analysis. Percept Psychophys, 56(1), 42-52. 
Heinze, H. J., Mangun, G. R., Burchert, W., Hinrichs, H., Scholz, M., Munte, T. F., et al. (1994). Combined spatial and temporal imaging of brain activity during visual selective attention in humans. Nature, 372(6506), 543-546.

Henderson, J. M., \& Macquistan, A. D. (1993). The spatial distribution of attention following an exogenous cue. Percept Psychophys, 53(2), 221-230.

Hillyard, S. A., \& Anllo-Vento, L. (1998). Event-related brain potentials in the study of visual selective attention. Proc Natl Acad Sci U S A, 95(3), 781-787.

Hoffman, E. A., \& Haxby, J. V. (2000). Distinct representations of eye gaze and identity in the distributed human neural system for face perception. Nat Neurosci, 3(1), 80-84.

Hoffman, J. E., \& Nelson, B. (1981). Spatial selectivity in visual search. Percept Psychophys, 30(3), 283-290.

Hoffman, J. E., Nelson, B., \& Houck, M. R. (1983). The role of attentional resources in automatic detection. Cognit Psychol, 15(3), 379-410.

Hoffman, K. L., Gothard, K. M., Schmid, M. C., \& Logothetis, N. K. (2007). Facialexpression and gaze-selective responses in the monkey amygdala. Curr Biol, 17(9), 766-772.

Hoge, R. D., \& Pike, G. B. (2001). Quantitative measurement using fMRI. In P. Jezzard, P. M. Matthews \& S. M. Smith (Eds.), Functional MRI (pp. 159-175). New York: Oxford University Press.

Holland, P. C., \& Gallagher, M. (2004). Amygdala-frontal interactions and reward expectancy. Current Opinion in Neuribiology, 14, 148-155.

Holmes, A., Kiss, M., \& Eimer, M. (2006). Attention modulates the processing of emotional expression triggered by foveal faces. Neurosci Lett, 394(1), 48-52.

Holmes, A., Vuilleumier, P., \& Eimer, M. (2003). The processing of emotional facial expression is gated by spatial attention: evidence from event-related brain potentials. Brain Res Cogn Brain Res, 16(2), 174-184.

Hopfinger, J. B., Buonocore, M. H., \& Mangun, G. R. (2000). The neural mechanisms of top-down attentional control. Nat Neurosci, 3(3), 284-291.

Hopfinger, J. B., Jha, A. P., Hopf, J. M., Girelli, M., \& Mangun, G. R. (2000). Electrophysiological and neuroimaging studies of voluntary and reflexive attention. In S. Monsell, Driver, J. (Ed.), Attention and Performance XVII (pp. 125-153). Cambrigde MA: MIT Press. 
Hornak, J., Rolls, E. T., \& Wade, D. (1996). Face and voice expression identification in patients with emotional and behavioural changes following ventral frontal lobe damage. Neuropsychologia, 34(4), 247-261.

Hsu, S. M., \& Pessoa, L. (2007). Dissociable effects of bottom-up and top-down factors on the processing of unattended fearful faces. Neuropsychologia, 45(13), 3075-3086.

Huettel, S. A., Song, A. W., \& McCarthy, G. (2004). Functional Magnteic Resonance Imaging. Sunderland, MA: Sinauer.

Huk, A. C., Dougherty, R. F., \& Heeger, D. J. (2002). Retinotopy and functional subdivision of human areas MT and MST. J Neurosci, 22(16), 7195-7205.

Huk, A. C., \& Heeger, D. J. (2002). Pattern-motion responses in human visual cortex. Nat Neurosci, 5(1), 72-75.

Iacoboni, M. (2005). Neural mechanisms of imitation. Curr Opin Neurobiol, 15(6), 632637.

Iacoboni, M., Woods, R. P., Brass, M., Bekkering, H., Mazziotta, J. C., \& Rizzolatti, G. (1999). Cortical mechanisms of human imitation. Science, 286(5449), 2526-2528.

Iidaka, T., Omori, M., Murata, T., Kosaka, H., Yonekura, Y., Okada, T., et al. (2001). Neural interaction of the amygdala with the prefrontal and temporal cortices in the processing of facial expressions as revealed by fMRI. J Cogn Neurosci, 13(8), 1035-1047.

Intriligator, J., \& Cavanagh, P. (2001). The spatial resolution of visual attention. Cognit Psychol, 43(3), 171-216.

Ishai, A., Haxby, J. V., \& Ungerleider, L. G. (2002). Visual imagery of famous faces: effects of memory and attention revealed by fMRI. Neuroimage, 17(4), 1729-1741.

Ishai, A., Schmidt, C. F., \& Boesiger, P. (2005). Face perception is mediated by a distributed cortical network. Brain Res Bull, 67(1-2), 87-93.

Ishai, A., Ungerleider, L. G., \& Haxby, J. V. (2000). Distributed neural systems for the generation of visual images. Neuron, 28(3), 979-990.

Ishai, A., Ungerleider, L. G., Martin, A., \& Haxby, J. V. (2000). The representation of objects in the human occipital and temporal cortex. J Cogn Neurosci, 12 Suppl 2, $35-51$.

Ishai, A., Ungerleider, L. G., Martin, A., Schouten, J. L., \& Haxby, J. V. (1999). Distributed representation of objects in the human ventral visual pathway. Proc Natl Acad Sci U S A, 96(16), 9379-9384. 
Jackson, M. E., \& Moghaddam, B. (2001). Amygdala regulation of nucleus accumbens dopamine output is governed by the prefrontal cortex. J Neurosci, 21(2), 676-681.

Jacobs, D. H., Shuren, J., Bowers, D., \& Heilman, K. M. (1995a). Emotional facial imagery, perception, and expression in Parkinson's disease. Neurology, 45(9), 1696-1702.

Jacobs, D. H., Shuren, J., \& Heilman, K. M. (1995b). Impaired perception of facial identity and facial affect in Huntington's disease. Neurology, 45(6), 1217-1218.

James, W. (1890/1950). The Principles of Psychology (New York: Dover).

James, W. (1950). The Principles of Psychology. New York: Dover.

Jezzard, P., Matthews, P. M., \& Smith, S. M. (2001). Functional MRI. An introduction to Methods. New York: Oxford University Press.

Johnson, S. A., Stout, J. C., Solomon, A. C., Langbehn, D. R., Aylward, E. H., Cruce, C. B., et al. (2007). Beyond disgust: impaired recognition of negative emotions prior to diagnosis in Huntington's disease. Brain, 130(Pt 7), 1732-1744.

Johnston, W. A., \& Heinz, S. P. (1978). Flexibility and capacity demands of attention. Journal of Experimental Psychology: General, 107, 420-435.

Johnstone, T., Somerville, L. H., Alexander, A. L., Oakes, T. R., Davidson, R. J., Kalin, N. H., et al. (2005). Stability of amygdala BOLD response to fearful faces over multiple scan sessions. Neuroimage, 25(4), 1112-1123.

Kahneman, D. (1973). Attention and Effort. Englewood Cliffs, NJ: Prentice-Hall.

Kalin, N. H., Shelton, S. E., Davidson, R. J., \& Kelley, A. E. (2001). The primate amygdala mediates acute fear but not the behavioral and physiological components of anxious temperament. J Neurosci, 21(6), 2067-2074.

Kandel, E. R., Svhwartz, J. H., \& Jessell, T. M. (2000). Principles of Neural Sciene (4th ed.). New York: McGraw-Hill.

Kanwisher, N. (2000). Domain specificity in face perception. Nat Neurosci, 3(8), 759-763.

Kanwisher, N., McDermott, J., \& Chun, M. M. (1997). The fusiform face area: a module in human extrastriate cortex specialized for face perception. J Neurosci, 17(11), 43024311.

Kanwisher, N., \& Wojciulik, E. (2000). Visual attention: insights from brain imaging. Nat Rev Neurosci, 1(2), 91-100.

Kastner, S., De Weerd, P., Desimone, R., \& Ungerleider, L. G. (1998). Mechanisms of directed attention in the human extrastriate cortex as revealed by functional MRI. Science, 282(5386), 108-111. 
Kastner, S., Pinsk, M. A., De Weerd, P., Desimone, R., \& Ungerleider, L. G. (1999). Increased activity in human visual cortex during directed attention in the absence of visual stimulation. Neuron, 22(4), 751-761.

Kastner, S., \& Ungerleider, L. G. (2000). Mechanisms of visual attention in the human cortex. Annu Rev Neurosci, 23, 315-341.

Kastner, S., \& Ungerleider, L. G. (2001). The neural basis of biased competition in human visual cortex. Neuropsychologia, 39(12), 1263-1276.

Kawasaki, H., Adolphs, R., Kaufman, O., Damasio, H., Damasio, A., Granner, M., et al. (2001). Single-unit responses to emotional visual stimuli recorded in human ventral prefrontal cortex. Nat Neurosci, 4, 15-16.

Kawasaki, H., Adolphs, R., Oya, H., Kovach, C., Damasio, H., Kaufman, O., et al. (2005). Analysis of single-unit responses to emotional scenes in human ventromedial prefrontal cortex. J Cogn Neurosci, 17(10), 1509-1518.

Kawashima, R., Sugiura, M., Kato, T., Nakamura, A., Hatano, K., Ito, K., et al. (1999). The human amygdala plays an important role in gaze monitoring. A PET study. Brain, 122 ( Pt 4), 779-783.

Keane, J., Calder, A. J., Hodges, J. R., \& Young, A. W. (2002). Face and emotion processing in frontal variant frontotemporal dementia. Neuropsychologia, 40(6), 655-665.

Kelley, T. A., Serences, J. T., Giesbrecht, B., \& Yantis, S. (2008). Cortical mechanisms for shifting and holding visuospatial attention. Cereb Cortex, 18(1), 114-125.

Kerns, J. G., Cohen, J. D., MacDonald, A. W., 3rd, Cho, R. Y., Stenger, V. A., \& Carter, C. S. (2004). Anterior cingulate conflict monitoring and adjustments in control. Science, 303(5660), 1023-1026.

Kesler-West, M. L., Andersen, A. H., Smith, C. D., Avison, M. J., Davisa, C. E., Kryscio, R. J., et al. (2001). Neural substrates of facial emotion processing using fMRI. Cognitive Brain Research, 11, 213-226.

Kiehl, K. A., Liddle, P. F., \& Hopfinger, J. B. (2000). Error processing and the rostral anterior cingulate: an event-related fMRI study. Psychophysiology, 37(2), 216-223.

Killgore, W. D., Oki, M., \& Yurgelun-Todd, D. A. (2001a). Sex-specific developmental changes in amygdala responses to affective faces. Neuroreport, 12(2), 427-433.

Killgore, W. D., \& Yurgelun-Todd, D. A. (2001b). Sex differences in amygdala activation during the perception of facial affect. Neuroreport, 12(11), 2543-2547. 
Killgore, W. D., \& Yurgelun-Todd, D. A. (2004). Activation of the amygdala and anterior cingulate during nonconscious processing of sad versus happy faces. Neuroimage, 21(4), 1215-1223.

Koch, M., Schmid, A., \& Schnitzler, H. U. (1996). Pleasure-attenuation of startle is disrupted by lesions of the nucleus accumbens. Neuroreport, 7(8), 1442-1446.

Kramer, A. F., \& Hahn, S. (1995). Splitting the beam: distribution of attention over noncontiguous regions of the visual field. Psychological Science, 6, 381-386.

Krolak-Salmon, P., Henaff, M. A., Vighetto, A., Bertrand, O., \& Mauguiere, F. (2004). Early amygdala reaction to fear spreading in occipital, temporal, and frontal cortex: a depth electrode ERP study in human. Neuron, 42(4), 665-676.

Kwak, H. W., Dagenbach, D., \& Egeth, H. (1991). Further evidence for a timeindependent shift of the focus of attention. Percept Psychophys, 49(5), 473-480.

Kwong, K. K., Belliveau, J. W., Chesler, D. A., Goldberg, I. E., Weisskoff, R. M., Poncelet, B. P., et al. (1992). Dynamic magnetic resonance imaging of human brain activity during primary sensory stimulation. Proc Natl Acad Sci U S A, 89(12), $5675-5679$.

LaBerge, D. (1983). Spatial extent of attention to letters and words. J Exp Psychol Hum Percept Perform, 9(3), 371-379.

LaBerge, D. (1995). Computational and anatomical models of selective attention in object identification. In M. Gazzaniga (Ed.), The cognive neurosciences. Cambridge, MA: MIT Press.

Lachenal-Chevallet, K., Bediou, B., Bouvard, M., Thobois, S., Broussolle, E., Vighetto, A., et al. (2006). [Emotional facial expression recognition impairment in Parkinson disease]. Psychol Neuropsychiatr Vieil, 4(1), 61-67.

Lane, R. D., Chua, P. M., \& Dolan, R. J. (1999). Common effects of emotional valence, arousal and attention on neural activation during visual processing of pictures. Neuropsychologia, 37(9), 989-997.

Lane, R. D., Reiman, E. M., Ahern, G. L., Schwartz, G. E., \& Davidson, R. J. (1997). Neuroanatomical correlates of happiness, sadness, and disgust. Am J Psychiatry, 154(7), 926-933.

Lauterbur, P. (1973). Image formation by induced local interactions: Examples employing nuclear magnetic resonance. Nature, 242(190-191).

Lavie, N. (1995). Perceptual load as a necessary condition for selective attention. J Exp Psychol Hum Percept Perform, 21(3), 451-468. 
Lavie, N. (2005). Distracted and confused?: selective attention under load. Trends Cogn Sci, 9(2), 75-82.

Lavie, N., \& Cox, S. (1997). On the efficiency of attentional selection: Efficient visual search results in inefficient rejection of distraction. Psychol. Sci., 8, 395-398.

Lavie, N., \& Fox, E. (2000). The role of perceptual load in negative priming. J Exp Psychol Hum Percept Perform, 26(3), 1038-1052.

LeDoux, J. (1996). The Emotional Brain: The Mysterious Underspinnings of Emotional Life. New York: Touchstone Press.

LeDoux, J. (2000). Emotion circuits in the brain. Annu Rev Neurosci, 23, 155-184.

LeDoux, J. E., Sakaguchi, A., \& Reis, D. J. (1984). Subcortical efferent projections of the medial geniculate nucleus mediate emotional responses conditioned to acoustic stimuli. J Neurosci, 4(3), 683-698.

Lee, T. M., Liu, H. L., Hoosain, R., Liao, W. T., Wu, C. T., Yuen, K. S., et al. (2002). Gender differences in neural correlates of recognition of happy and sad faces in humans assessed by functional magnetic resonance imaging. Neurosci Lett, 333(1), $13-16$.

Leventhal, A. G., Rodieck, R. W., \& Dreher, B. (1985). Central projections of cat retinal ganglion cells. J Comp Neurol, 237(2), 216-226.

Levy, I., Hasson, U., Avidan, G., Hendler, T., \& Malach, R. (2001). Center-periphery organization of human object areas. Nat Neurosci, 4(5), 533-539.

Liddell, B. J., Brown, K. J., Kemp, A. H., Barton, M. J., Das, P., Peduto, A., et al. (2005). A direct brainstem-amygdala-cortical 'alarm' system for subliminal signals of fear. Neuroimage, 24(1), 235-243.

Liu, C. H., Collin, C. A., Rainville, S. J., \& Chaudhuri, A. (2000). The effects of spatial frequency overlap on face recognition. J Exp Psychol Hum Percept Perform, 26(3), 956-979.

Liu, L., \& Ioannides, A. A. (2006). Spatiotemporal dynamics and connectivity pattern differences between centrally and peripherally presented faces. Neuroimage, 31(4), 1726-1740.

Liu, L., Ioannides, A. A., \& Streit, M. (1999). Single trial analysis of neurophysiological correlates of the recognition of complex objects and facial expressions of emotion. Brain Topogr, 11(4), 291-303.

Livingstone, M., \& Hubel, D. (1988). Segregation of form, color, movement, and depth: anatomy, physiology, and perception. Science, 240(4853), 740-749. 
Livingstone, M. S., \& Hubel, D. H. (1987). Psychophysical evidence for separate channels for the perception of form, color, movement, and depth. J Neurosci, 7(11), 34163468.

Lu, Z. L., \& Dosher, B. A. (1998). External noise distinguishes attention mechanisms. Vision Res, 38(9), 1183-1198.

Luck, S. J., Chelazzi, L., Hillyard, S. A., \& Desimone, R. (1997). Neural mechanisms of spatial selective attention in areas $\mathrm{V} 1, \mathrm{~V} 2$, and V4 of macaque visual cortex. $J$ Neurophysiol, 77(1), 24-42.

Luck, S. J., \& Ford, M. A. (1998). On the role of selective attention in visual perception. Proc Natl Acad Sci U S A, 95(3), 825-830.

Luck, S. J., \& Hillyard, S. A. (1995). The role of attention in feature detection and conjunction discrimination: an electrophysiological analysis. Int J Neurosci, 80(14), 281-297.

Luck, S. J., Woodman, G. F., \& Vogel, E. K. (2000). Event-related potential studies of attention. Trends Cogn Sci, 4(11), 432-440.

Lundqvist, D., Flykt, A., \& Ohman, A. (1998). The Karolinska Directed Emotional Faces (KDEF). Stockholm: Karolinska Institute.

Luppino, G., Rozzi, S., Calzavara, R., \& Matelli, M. (2003). Prefrontal and agranular cingulate projections to the dorsal premotor areas F2 and F7 in the macaque monkey. Eur J Neurosci, 17(3), 559-578.

MacDonald, A. W., 3rd, Cohen, J. D., Stenger, V. A., \& Carter, C. S. (2000). Dissociating the role of the dorsolateral prefrontal and anterior cingulate cortex in cognitive control. Science, 288(5472), 1835-1838.

Magistretti, P. J., \& Pellerin, L. (1999). Cellular mechanisms of brain energy metabolism and their relevance to functional brain imaging. Philos Trans $R$ Soc Lond B Biol Sci, 354(1387), 1155-1163.

Makela, P., Nasanen, R., Rovamo, J., \& Melmoth, D. (2001). Identification of facial images in peripheral vision. Vision Res, 41(5), 599-610.

Malach, R., Levy, I., \& Hasson, U. (2002). The topography of high-order human object areas. Trends Cogn Sci, 6(4), 176-184.

Malinowski, P., Fuchs, S., \& Muller, M. M. (2007). Sustained division of spatial attention to multiple locations within one hemifield. Neurosci Lett, 414(1), 65-70.

Mangun, G. R. (1995). Neural mechanisms of visual selective attention. Psychophysiology, 32(1), 4-18. 
Marinkovic, K., Trebon, P., Chauvel, P., \& Halgren, E. (2000). Localised face processing by the human prefrontal cortex: Face-selective intracerebral potentials and postlesion deficits. Cognitive Neuropsychology, 17, 187-199.

Martinez, A., Anllo-Vento, L., Sereno, M. I., Frank, L. R., Buxton, R. B., Dubowitz, D. J., et al. (1999). Involvement of striate and extrastriate visual cortical areas in spatial attention. Nat Neurosci, 2(4), 364-369.

Maunsell, J. H. (1995). The brain's visual world: representation of visual targets in cerebral cortex. Science, 270(5237), 764-769.

Mazoyer, B., Zago, L., Mellet, E., Bricogne, S., Etard, O., Houde, O., et al. (2001). Cortical networks for working memory and executive functions sustain the conscious resting state in man. Brain Res Bull, 54(3), 287-298.

McAdams, C. J., \& Maunsell, J. H. (1999). Effects of attention on orientation-tuning functions of single neurons in macaque cortical area V4. J Neurosci, 19(1), 431441.

McCarthy, G., Puce, A., Gore, J. C., \& Allison, T. (1997). Face-Specific processing in the human fusiform gyrus. Journal of Cognitive Neuroscience, 9(5), 605-610.

McClure, E. B., Monk, C. S., Nelson, E. E., Zarahn, E., Leibenluft, E., Bilder, R. M., et al. (2004). A developmental examination of gender differences in brain engagement during evaluation of threat. Biol Psychiatry, 55(11), 1047-1055.

McCormick, P. A., \& Klein, R. (1990). The spatial distribution of attention during covert visual orienting. Acta Psychol (Amst), 75(3), 225-242.

McCormick, P. A., Klein, R. M., \& Johnston, S. (1998). Splitting versus sharing focal attention: comment on Castiello and Umilta (1992). J Exp Psychol Hum Percept Perform, 24(1), 350-357.

McGonigle, D. J., Howseman, A. M., Athwal, B. S., Friston, K. J., Frackowiak, R. S., \& Holmes, A. P. (2000). Variability in fMRI: an examination of intersession differences. Neuroimage, 11(6 Pt 1), 708-734.

McMains, S. A., \& Somers, D. C. (2004). Multiple spotlights of attentional selection in human visual cortex. Neuron, 42(4), 677-686.

McMains, S. A., \& Somers, D. C. (2005). Processing efficiency of divided spatial attention mechanisms in human visual cortex. J Neurosci, 25(41), 9444-9448.

Mega, M. S., Cummings, J. L., Salloway, S., \& Malloy, P. (1997). The limbic system: an anatomic, phylogenetic, and clinical perspective. J Neuropsychiatry Clin Neurosci, 9(3), 315-330. 
Menon, V., Adleman, N. E., White, C. D., Glover, G. H., \& Reiss, A. L. (2001). Errorrelated brain activation during a Go/NoGo response inhibition task. Hum Brain Mapp, 12(3), 131-143.

Merigan, W. H., \& Maunsell, J. H. (1993). How parallel are the primate visual pathways? Annu Rev Neurosci, 16, 369-402.

Miezin, F. M., Maccotta, L., Ollinger, J. M., Petersen, S. E., \& Buckner, R. L. (2000). Characterizing the hemodynamic response: effects of presentation rate, sampling procedure, and the possibility of ordering brain activity based on relative timing. Neuroimage, 11(6 Pt 1), 735-759.

Milham, M. P., \& Banich, M. T. (2005). Anterior cingulate cortex: an fMRI analysis of conflict specificity and functional differentiation. Hum Brain Mapp, 25(3), 328335.

Milham, M. P., Banich, M. T., \& Barad, V. (2003). Competition for priority in processing increases prefrontal cortex's involvement in top-down control: an event-related fMRI study of the stroop task. Brain Res Cogn Brain Res, 17(2), 212-222.

Milham, M. P., Banich, M. T., Webb, A., Barad, V., Cohen, N. J., Wszalek, T., et al. (2001). The relative involvement of anterior cingulate and prefrontal cortex in attentional control depends on nature of conflict. Brain Res Cogn Brain Res, 12(3), 467-473.

Miller, J. (1991). The flanker compatibility effect as a function of visual angle, attentional focus, visual transients, and perceptual load: a search for boundary conditions. Percept Psychophys, 49(3), 270-288.

Mishkin, M., Ungerleider, L. G., \& Macko, K. A. (1983). Object vision and spatial vision: two cortical pathways. Trends Neurosci., 6, 414-417.

Mitchell, D. G. V., Nakic, M., Fridberg, D., Kamel, N., Pine, D. S., \& Blair, R. J. R. (2007). The impact of prcessing load on emotion. Neuroimage, 34, 1299-1309.

Mitchell, D. G. V., Nakic, M., Fridberg, D., Kamel, N., Pine, D. S., \& Blair, R. J. R. (2007). The impact of prcessing load on emotion. Neuroimage, 34, 1299-1309.

Mitchell, J. P. (2008). Activity in right temporo-parietal junction is not selective for theory-of-mind. Cereb Cortex, 18(2), 262-271.

Mohanty, A., Engels, A. S., Herrington, J. D., Heller, W., Ho, M. H., Banich, M. T., et al. (2007). Differential engagement of anterior cingulate cortex subdivisions for cognitive and emotional function. Psychophysiology, 44(3), 343-351. 
Moran, J., \& Desimone, R. (1985). Selective attention gates visual processing in the extrastriate cortex. Science, 229(4715), 782-784.

Morawetz, C., Holz, P., Baudewig, J., Treue, S., \& Dechent, P. (2007). Split of attentional resources in human visual cortex. Vis Neurosci, 24(6), 817-826.

Morris, J. S., deBonis, M., \& Dolan, R. J. (2002). Human amygdala responses to fearful eyes. Neuroimage, 17(1), 214-222.

Morris, J. S., DeGelder, B., Weiskrantz, L., \& Dolan, R. J. (2001). Differential extrageniculostriate and amygdala responses to presentation of emotional faces in a cortically blind field. Brain, 124(Pt 6), 1241-1252.

Morris, J. S., Friston, K. J., Buchel, C., Frith, C. D., Young, A. W., Calder, A. J., et al. (1998a). A neuromodulatory role for the human amygdala in processing emotional facial expressions. Brain, 121 ( Pt 1), 47-57.

Morris, J. S., Frith, C. D., Perrett, D. I., Rowland, D., Young, A. W., Calder, A. J., et al. (1996a). A differential neural response in the human amygdala to fearful and happy facial expressions. Nature, 383(6603), 812-815.

Morris, J. S., Ohman, A., \& Dolan, R. J. (1998b). Conscious and unconscious emotional learning in the human amygdala. Nature, 393(6684), 467-470.

Morris, J. S., Ohman, A., \& Dolan, R. J. (1999). A subcortical pathway to the right amygdala mediating "unseen" fear. Proc Natl Acad Sci U S A, 96(4), 1680-1685.

Morris, P. L., Robinson, R. G., de Carvalho, M. L., Albert, P., Wells, J. C., Samuels, J. F., et al. (1996b). Lesion characteristics and depressed mood in the stroke data bank study. J Neuropsychiatry Clin Neurosci, 8(2), 153-159.

Motter, B. C. (1994). Neural correlates of attentive selection for color or luminance in extrastriate area V4. J Neurosci, 14(4), 2178-2189.

Mounts, J. R. (2005). Attentional selection: A salience-based competition for representation. Percept Psychophys, 67(7), 1190-1198.

Mueller, M. M., Malinowski, P., Gruber, T., \& Hillyard, S. A. (2003a). Sustained division of the attentional spotlight. Nature, 424, 309-312.

Mueller, N. G., Bartelt, O., Donner, T. H., Villringer, A., \& Brandt, S. A. (2003b). A physiological correlate of the „zoom lens“ of visual attention. The Journal of Neuroscience, 23(9), 3561-3565.

Muller, M. M., Malinowski, P., Gruber, T., \& Hillyard, S. A. (2003b). Sustained division of the attentional spotlight. Nature, 424(6946), 309-312. 
Muller, N. G., Bartelt, O. A., Donner, T. H., Villringer, A., \& Brandt, S. A. (2003a). A physiological correlate of the "Zoom Lens" of visual attention. J Neurosci, 23(9), 3561-3565.

Murphy, F. C., Nimmo-Smith, I., \& Lawrence, A. D. (2003). Functional neuroanatomy of emotions: a meta-analysis. Cogn Affect Behav Neurosci, 3(3), 207-233.

Murphy, S. T., Monahan, J. L., \& Zajonc, R. B. (1995). Additivity of nonconscious affect: combined effects of priming and exposure. J Pers Soc Psychol, 69(4), 589-602.

Murphy, S. T., \& Zajonc, R. B. (1993). Affect, cognition, and awareness: affective priming with optimal and suboptimal stimulus exposures. J Pers Soc Psychol, 64(5), 723 739.

Murphy, T. D., \& Eriksen, C. W. (1987). Temporal changes in the distribution of attention in the visual field in response to precues. Percept Psychophys, 42(6), 576-586.

Nakamura, K., Kawashima, R., Ito, K., Sugiura, M., Kato, T., Nakamura, A., et al. (1999). Activation of the right inferior frontal cortex during assessment of facial emotion. $J$ Neurophysiol, 82(3), 1610-1614.

Narumoto, J., Okada, T., Sadato, N., Fukui, K., \& Yonekura, Y. (2001). Attention to emotion modulates fMRI activity in human right superior temporal sulcus. Brain Res Cogn Brain Res, 12(2), 225-231.

Narumoto, J., Yamada, H., Iidaka, T., Sadato, N., Fukui, K., Itoh, H., et al. (2000). Brain regions involved in verbal or non-verbal aspects of facial emotion recognition. Neuroreport, 11(11), 2571-2576.

Nielsen, A. F. (2003). The Brede database: a small database for functional neuroimaging. NeuroImage, 19(2), Presented at the 9th International Conference on Functional Mapping of the Human Brain, June 19--22, 2003, New York, NY.

Nomura, M., Ohira, H., Haneda, K., Iidaka, T., Sadato, N., Okada, T., et al. (2004). Functional association of the amygdala and ventral prefrontal cortex during cognitive evaluation of facial expressions primed by masked angry faces: an eventrelated fMRI study. Neuroimage, 21(1), 352-363.

Norman, J., \& Ehrlich, S. (1987). Spatial frequency filtering and target identification. Vision Res, 27(1), 87-96.

Ochsner, K. N., Bunge, S. A., Gross, J. J., \& Gabrieli, J. D. (2002). Rethinking feelings: an FMRI study of the cognitive regulation of emotion. J Cogn Neurosci, 14(8), 12151229. 
Ochsner, K. N., \& Gross, J. J. (2005). The cognitive control of emotion. Trends Cogn Sci, 9(5), 242-249.

O'Craven, K. M., Downing, P. E., \& Kanwisher, N. (1999). fMRI evidence for objects as the units of attentional selection. Nature, 401(6753), 584-587.

O'Craven, K. M., \& Kanwisher, N. (2000). Mental imagery of faces and places activates corresponding stiimulus-specific brain regions. J Cogn Neurosci, 12(6), 1013-1023.

O'Craven, K. M., Rosen, B. R., Kwong, K. K., Treisman, A., \& Savoy, R. L. (1997). Voluntary attention modulates fMRI activity in human MT-MST. Neuron, 18(4), 591-598.

Ogawa, S., Lee, T. M., Kay, A. R., \& Tank, D. W. (1990). Brain magnetic resonance imaging with contrast dependent on blood oxygenation. Proc Natl Acad Sci U S A, 87(24), 9868-9872.

Ogawa, S., Tank, D. W., Menon, R., Ellermann, J. M., Kim, S. G., Merkle, H., et al. (1992). Intrinsic signal changes accompanying sensory stimulation: functional brain mapping with magnetic resonance imaging. Proc Natl Acad Sci U $S$ A, 89(13), 5951-5955.

Öhman, A. (2002). Automaticity and the Amygdala: Nonconsious Responses to Emotional Faces. Current Directions in Psychological Science, 11(2), 62-66.

Öhman, A. (2005). The role of the amygdala in human fear: Automatic detection of threat. Psychoneuroendocrinology, 30, 953-958.

Öhman, A., Carlsson, K., Lundquist, D., \& Ingvar, M. (2007). On the unconsious subcortical origin of human fear. Physiology \& Behavior, 92, 180-185.

Ojemann, J. G., Ojemann, G. A., \& Lettich, E. (1992). Neuronal activity related to faces and matching in human right nondominant temporal cortex. Brain, 115 Pt 1, 1-13.

Öngür, D., \& Price, J. L. (2000). The organization of networks within the orbital and medial prefrontal cortex of rats, monkeys and humans. Cereb Cortex, 10, 206-219.

Palermo, R., \& Rhodes, G. (2007). Are you always on my mind? A review of how face perception and attention interact. Neuropsychologia, 45(1), 75-92.

Pan, K., \& Eriksen, C. W. (1993). Attentional distribution in the visual field during samedifferent judgments as assessed by response competition. Percept Psychophys, 53(2), 134-144.

Pashler, H. E. (1998). The psychology of attention. Cambridge, MA: MIT Press.

Pasley, B. N., Mayes, L. C., \& Schultz, R. T. (2004). Subcortical discrimination of unperceived objects during binocular rivalry. Neuron, 42(1), 163-172. 
Pauling, L., \& Coryell, C. D. (1936). The magnetic properties and structure of hemoglobin, oxyhemoglobin and caronmonoxyhemoglobin. Proceedings of National Academy of Sciences USA, 22, 210-216.

Paus, T. (2001). Primate anterior cingulate cortex: where motor control, drive and cognition interface. Nat Rev Neurosci, 2(6), 417-424.

Pell, M. D., \& Leonard, C. L. (2005). Facial expression decoding in early Parkinson's disease. Brain Res Cogn Brain Res, 23(2-3), 327-340.

Perrett, D. I., Harries, M. F., Mistlin, A., Hietanen, J. K., Benson, P. J., \& Bevan, R. (1990). Social signals analyzed at the single cell level: Someone is looking at me, something touched me, something moved! Int J Comp Psychol, 4, 25-55.

Perrett, D. I., Hietanen, J. K., Oram, M. W., \& Benson, P. J. (1992). Organization and functions of cells responsive to faces in the temporal cortex. Philos Trans $R$ Soc Lond B Biol Sci, 335(1273), 23-30.

Perrett, D. I., Smith, P. A., Potter, D. D., Mistlin, A. J., Head, A. S., Milner, A. D., et al. (1984). Neurones responsive to faces in the temporal cortex: studies of functional organization, sensitivity to identity and relation to perception. Hum Neurobiol, 3(4), 197-208.

Perrett, D. I., Smith, P. A., Potter, D. D., Mistlin, A. J., Head, A. S., Milner, A. D., et al. (1985). Visual cells in the temporal cortex sensitive to face view and gaze direction. Proc R Soc Lond B Biol Sci, 223(1232), 293-317.

Pessoa, L. (2005a). To what extent are emotional visual stimuli processed without attention and awareness? Curr Opin Neurobiol, 15(2), 188-196.

Pessoa, L. (2008). On the relationship between emotion and cognition. Nat Rev Neurosci, 9(2), 148-158.

Pessoa, L., Japee, S., Sturman, D., \& Ungerleider, L. G. (2006). Target visibility and visual awareness modulate amygdala responses to fearful faces. Cereb Cortex, 16(3), 366375.

Pessoa, L., McKenna, M., Gutierrez, E., \& Ungerleider, L. G. (2002). Neural processing of emotional faces requires attention. Proc Natl Acad Sci U S A, 99(17), 11458-11463.

Pessoa, L., Padmala, S., \& Morland, T. (2005b). Fate of unattended fearful faces in the amygdala is determined by both attentional resources and cognitive modulation. Neuroimage, 28(1), 249-255.

Peterson, M. S., \& Juola, J. F. (2000). Evidence for distinct attentional bottlenecks in attention switching and attentional blink tasks. J Gen Psychol, 127(1), 6-26. 
Petrovic, P., Carlsson, K., Petersson, K. M., Hansson, P., \& Ingvar, M. (2004). Contextdependent deactivation of the amygdala during pain. J Cogn Neurosci, 16(7), 12891301.

Phan, K. L., Wager, T., Taylor, S. F., \& Liberzon, I. (2002). Functional neuroanatomy of emotion: a meta-analysis of emotion activation studies in PET and fMRI. Neuroimage, 16(2), 331-348.

Phelps, E. A. (2006). Emotion and cognition: insights from studies of the human amygdala. Annu Rev Psychol, 57, 27-53.

Phelps, E. A., \& LeDoux, J. E. (2005). Contributions of the amygdala to emotion processing: from animal models to human behavior. Neuron, 48(2), 175-187.

Phelps, E. A., O'Connor, K. J., Cunningham, W. A., Funayama, E. S., Gatenby, J. C., Gore, J. C., et al. (2000). Performance on indirect measures of race evaluation predicts amygdala activation. J Cogn Neurosci, 12(5), 729-738.

Phillips, M. L., Bullmore, E. T., Howard, R., Woodruff, P. W., Wright, I. C., Williams, S. C., et al. (1998b). Investigation of facial recognition memory and happy and sad facial expression perception: an fMRI study. Psychiatry Res, 83(3), 127-138.

Phillips, M. L., Medford, N., Young, A. W., Williams, L., Williams, S. C., Bullmore, E. T., et al. (2001). Time courses of left and right amygdalar responses to fearful facial expressions. Hum Brain Mapp, 12(4), 193-202.

Phillips, M. L., Williams, L. M., Heining, M., Herba, C. M., Russell, T., Andrew, C., et al. (2004). Differential neural responses to overt and covert presentations of facial expressions of fear and disgust. Neuroimage, 21(4), 1484-1496.

Phillips, M. L., Young, A. W., Scott, S. K., Calder, A. J., Andrew, C., Giampietro, V., et al. (1998a). Neural responses to facial and vocal expressions of fear and disgust. Proc Biol Sci, 265(1408), 1809-1817.

Phillips, M. L., Young, A. W., Senior, C., Brammer, M., Andrew, C., Calder, A. J., et al. (1997). A specific neural substrate for perceiving facial expressions of disgust. Nature, 389(6650), 495-498.

Pierrot-Deseilligny, C., Ploner, C. J., Muri, R. M., Gaymard, B., \& Rivaud-Pechoux, S. (2002). Effects of cortical lesions on saccadic: eye movements in humans. Ann N Y Acad Sci, 956, 216-229.

Pizzagalli, D., Regard, M., \& Lehmann, D. (1999). Rapid emotional face processing in the human right and left brain hemispheres: an ERP study. Neuroreport, 10(13), 26912698. 
Ploner, C. J., Gaymard, B. M., Rivaud-Pechoux, S., \& Pierrot-Deseilligny, C. (2005). The prefrontal substrate of reflexive saccade inhibition in humans. Biol Psychiatry, 57(10), 1159-1165.

Posner, M. (1995). Neuropsychology. Modulation by instruction. Nature, 373(6511), 198199.

Posner, M., \& Petersen, S. E. (1999). The attention system of the human brain. Annu Rev Neurosci, 13, 25-42.

Posner, M., \& Petersen, S. E. (1999). The attention system of the human brain. Annu Rev Neurosci, 13, 25-42.

Posner, M. I. (1980). Orienting of attention. Q J Exp Psychol, 32(1), 3-25.

Posner, M. I., \& Boies, S. W. (1971). Components of attention. Psychological Review, 78, 391-408.

Posner, M. I., \& Cohen, Y. (1984). Components of visual orienting. In H. Bouman \& D. G. Bouwhuis (Eds.), Attention and Performance X. Hillsdale NJ: Erlbaum.

Posner, M. I., \& Petersen, S. E. (1990). The attention system of the human brain. Annu Rev Neurosci, 13, 25-42.

Posner, M. I., \& Rothbart, M. K. (2007). Research on attention networks as a model for the integration of psychological science. Annu Rev Psychol, 58, 1-23.

Posner, M. I., Snyder, C. R., \& Davidson, B. J. (1980). Attention and the detection of signals. J Exp Psychol, 109(2), 160-174.

Pourtois, G., Grandjean, D., Sander, D., \& Vuilleumier, P. (2004). Electrophysiological correlates of rapid spatial orienting towards fearful faces. Cereb Cortex, 14(6), 619633.

Pourtois, G., \& Vuilleumier, P. (2006). Dynamics of emotional effects on spatial attention in the human visual cortex. Prog Brain Res, 156, 67-91.

Puce, A., Allison, T., Asgari, M., Gore, J. C., \& McCarthy, G. (1996). Differential sensitivity of human visual cortex to faces, letterstrings, and textures: a functional magnetic resonance imaging study. J Neurosci, 16(16), 5205-5215.

Puce, A., Allison, T., Bentin, S., Gore, J. C., \& McCarthy, G. (1998). Temporal cortex activation in humans viewing eye and mouth movements. $J$ Neurosci, 18(6), 21882199.

Purcell, E. M., Torry, H. C., \& Pound, R. V. (1946). Resonance absorption by nuclear magnetic moments in a solid. Physiological Review, 69, 37. 
Quirk, G. J., Russo, G. K., Barron, J. L., \& Lebron, K. (2000). The role of ventromedial prefrontal cortex in the recovery of extinguished fear. $J$ Neurosci, 20(16), 62256231.

Raichle, M. E., \& Snyder, A. Z. (2007). A default mode of brain function: a brief history of an evolving idea. Neuroimage, 37(4), 1083-1090; discussion 1097-1089.

Rapcsak, S. Z., Kaszniak, A. W., \& Rubens, A. B. (1989). Anomia for facial expressions: evidence for a category specific visual-verbal disconnection syndrome. Neuropsychologia, 27(8), 1031-1041.

Rauch, S. L., Savage, C. R., Alpert, N. M., Fischman, A. J., \& Jenike, M. A. (1997). The functional neuroanatomy of anxiety: a study of three disorders using positron emission tomography and symptom provocation. Biol Psychiatry, 42(6), 446-452.

Rauch, S. L., Savage, C. R., Alpert, N. M., Miguel, E. C., Baer, L., Breiter, H. C., et al. (1995). A positron emission tomographic study of simple phobic symptom provocation. Arch Gen Psychiatry, 52(1), 20-28.

Reddy, L., Moradi, F., \& Koch, C. (2007). Top-down biases win against focal attention in the fusiform face area. Neuroimage, 38(4), 730-739.

Rees, G., Friston, K., \& Koch, C. (2000). A direct quantitative relationship between the functional properties of human and macaque V5. Nat Neurosci, 3(7), 716-723.

Rees, G., Frith, C. D., \& Lavie, N. (1997). Modulating irrelevant motion perception by varying attentional load in an unrelated task. Science, 278(5343), 1616-1619.

Reeves, A., \& Sperling, G. (1986). Attention gating in short-term visual memory. Psychol Rev, 93(2), 180-206.

Reeves, A., \& Sperling, G. (1986). Attention gating in short-term visual memory. Psychol Rev, 93(2), 180-206.

Remington, R., \& Pierce, L. (1984). Moving attention: evidence for time-invariant shifts of visual selective attention. Percept Psychophys, 35(4), 393-399.

Rizzolatti, G., \& Arbib, M. A. (1999). From grasping to speech: imitation might provide a missing link: reply. Trends Neurosci, 22(4), 152.

Rizzolatti, G., Fadiga, L., Gallese, V., \& Fogassi, L. (1996). Premotor cortex and the recognition of motor actions. Brain Res Cogn Brain Res, 3(2), 131-141.

Rizzolatti, G., Fogassi, L., \& Gallese, V. (2002). Motor and cognitive functions of the ventral premotor cortex. Curr Opin Neurobiol, 12(2), 149-154.

Rizzolatti, G., \& Luppino, G. (2001). The cortical motor system. Neuron, 31(6), 889-901. 
Robinson, R. G., \& Downhill, J. E. (1995). Lateralization of psychopathology in response to focal brain injury. In R. J. Davidson \& K. Hugdahl (Eds.), Brain Asymmetry (pp. 693-711): MIT Press.

Rolls, E. T., \& Tovee, M. J. (1994). Processing speed in the cerebral cortex and the neurophysiology of visual masking. Proc Biol Sci, 257(1348), 9-15.

Rotshtein, P., Malach, R., Hadar, U., Graif, M., \& Hendler, T. (2001). Feeling or features: different sensitivity to emotion in high-order visual cortex and amygdala. Neuron, 32(4), 747-757.

Rotshtein, P., Vuilleumier, P., Winston, J., Driver, J., \& Dolan, R. (2007). Distinct and convergent visual processing of high and low spatial frequency information in faces. Cereb Cortex, 17(11), 2713-2724.

Rotteveel, M., de Groot, P., Geutskens, A., \& Phaf, R. H. (2001). Stronger suboptimal than optimal affective priming? Emotion, 1(4), 348-364.

Salin, P. A., \& Bullier, J. (1995). Corticocortical connections in the visual system: structure and function. Physiol Rev, 75(1), 107-154.

Sato, W., Kochiyama, T., Yoshikawa, S., \& Matsumura, M. (2001). Emotional expression boosts early visual processing of the face: ERP recording and its decomposition by independent component analysis. Neuroreport, 12(4), 709-714.

Sato, W., Yoshikawa, S., Kochiyama, T., \& Matsumura, M. (2004). The amygdala processes the emotional significance of facial expressions: an fMRI investigation using the interaction between expression and face direction. Neuroimage, 22(2), 1006-1013.

Scharlau, I. (2004). Evidence for split foci of attention in a priming paradigm. Percept Psychophys, 66(6), 988-1002.

Schienle, A., Stark, R., Walter, B., Blecker, C., Ott, U., Kirsch, P., et al. (2002). The insula is not specifically involved in disgust processing: an fMRI study. Neuroreport, 13(16), 2023-2026.

Schiller, P. H., Malpeli, J. G., \& Schein, S. J. (1979). Composition of geniculostriate input ot superior colliculus of the rhesus monkey. J Neurophysiol, 42(4), 1124-1133.

Schmidt, W. C., Fisher, B. D., \& Pylyshyn, Z. W. (1998). Multiple-location access in vision: evidence from illusory line motion. J Exp Psychol Hum Percept Perform, 24(2), 505-525.

Schmolck, H., \& Squire, L. R. (2001). Impaired perception of facial emotions following bilateral damage to the anterior temporal lobe. Neuropsychology, 15(1), 30-38. 
Schneider, F., Grodd, W., Weiss, U., Klose, U., Mayer, K. R., Nagele, T., et al. (1997). Functional MRI reveals left amygdala activation during emotion. Psychiatry Res, 76(2-3), 75-82.

Schneider, F., Habel, U., Kessler, C., Salloum, J. B., \& Posse, S. (2000). Gender differences in regional cerebral activity during sadness. Hum Brain Mapp, 9(4), 226-238.

Schyns, P. G., \& Oliva, A. (1999). Dr. Angry and Mr. Smile: when categorization flexibly modifies the perception of faces in rapid visual presentations. Cognition, 69(3), 243-265.

Scott, S. K., Young, A. W., Calder, A. J., Hellawell, D. J., Aggleton, J. P., \& Johnson, M. (1997). Impaired auditory recognition of fear and anger following bilateral amygdala lesions. Nature, 385(6613), 254-257.

Serences, J. T., Shomstein, S., Leber, A. B., Golay, X., Egeth, H. E., \& Yantis, S. (2005). Coordination of voluntary and stimulus-driven attentional control in human cortex. Psychol Sci, 16(2), 114-122.

Sereno, M. I., Dale, A. M., Reppas, J. B., Kwong, K. K., Belliveau, J. W., Brady, T. J., et al. (1995). Borders of multiple visual areas in humans revealed by functional magnetic resonance imaging. Science, 268(5212), 889-893.

Sereno, M. I., Pitzalis, S., \& Martinez, A. (2001). Mapping of contralateral space in retinotopic coordinates by a parietal cortical area in humans. Science, 294(5545), $1350-1354$.

Sergent, J., Ohta, S., \& MacDonald, B. (1992). Functional neuroanatomy of face and object processing. A positron emission tomography study. Brain, 115 Pt 1, 15-36.

Sergerie, K., Chochol, C., \& Armony, J. L. (2008). The role of the amygdala in emotional processing: A quantitative meta-analysis of functional neuroimaging studies. Neurosci Biobehav Rev, 32(4), 811-830.

Shaw, M. L. (1978). A capacity allocation model for reaction time. Journal of Experimental Psychology: Human Perception \& Performance, 4, 586-598.

Shaw, M. L., \& Shaw, P. (1977). Optimal allocation of cognitive resources to spatial locations. J Exp Psychol Hum Percept Perform, 3(2), 201-211.

Shiffrin, R. M., \& Schneider, W. (1977). Controlled and automatic human information processing II Perceptual learning, automatic attending, and a general theory. Psychological Review, 84, 127-190. 
Shipp, S. (2001). Corticopulvinar connections of areas V5, V4, and V3 in the macaque monkey: a dual model of retinal and cortical topographies. J Comp Neurol, 439(4), 469-490.

Shiu, L. P., \& Pashler, H. (1995). Spatial attention and vernier acuity. Vision Res, 35(3), 337-343.

Shulman, G. L. (1990). Relating attention to visual mechanisms. Percept Psychophys, 47(2), 199-203.

Shulman, G. L., Corbetta, M., Buckner, R. L., Fiez, J. A., Miezen, F. M., Raichle, M. E., et al. (1997). Common blood flow changes across visual tasks: II. Decreases in cerebral cortex. Journal of Cognitive Neuroscience, 9(5), 647-662.

Shulman, G. L., Remington, R. W., \& McLean, J. P. (1979). Moving attention through visual space. J Exp Psychol Hum Percept Perform, 5(3), 522-526.

Silvert, L., Lepsien, J., Fragopanagos, N., Goolsby, B., Kiss, M., Taylor, J. G., et al. (2007). Influence of attentional demands on the processing of emotional facial expressions in the amygdala. Neuroimage, 38(2), 357-366.

Skinner, B. (1953). Science and Human Behavior. New York: Macmillan.

Slotnick, S. D., Schwarzbach, J., \& Yantis, S. (2003). Attentional inhibition of visual processing in human striate and extrastriate cortex. Neuroimage, 19(4), 1602-1611.

Somers, D. C., Dale, A. M., Seiffert, A. E., \& Tootell, R. B. (1999). Functional MRI reveals spatially specific attentional modulation in human primary visual cortex. Proc Natl Acad Sci U S A, 96(4), 1663-1668.

Sperling, G., \& Weichselgartner, E. (1995). Episodic theory of the dynamics of spatial attention. Psychological Review, 102(3), 503-532.

Spitzer, H., Desimone, R., \& Moran, J. (1988). Increased attention enhances both behavioral and neuronal performance. Science, 240(4850), 338-340.

Sprengelmeyer, R., Rausch, M., Eysel, U. T., \& Przuntek, H. (1998). Neural structures associated with recognition of facial expressions of basic emotions. Proc Biol Sci, 265(1409), 1927-1931.

Sprengelmeyer, R., Young, A. W., Calder, A. J., Karnat, A., Lange, H., Homberg, V., et al. (1996). Loss of disgust. Perception of faces and emotions in Huntington's disease. Brain, 119 ( Pt 5), 1647-1665.

Sprengelmeyer, R., Young, A. W., Pundt, I., Sprengelmeyer, A., Calder, A. J., Berrios, G., et al. (1997). Disgust implicated in obsessive-compulsive disorder. Proc Biol Sci, 264(1389), 1767-1773. 
Stefanacci, L., \& Amaral, D. G. (2002). Some observations on cortical inputs to the macaque monkey amygdala: an anterograde tracing study. J Comp Neurol, 451(4), 301-323.

Strafella, A. P., \& Paus, T. (2000). Modulation of cortical excitability during action observation: a transcranial magnetic stimulation study. Neuroreport, 11(10), 22892292.

Streit, M., Dammers, J., Simsek-Kraues, S., Brinkmeyer, J., Wolwer, W., \& Ioannides, A. (2003). Time course of regional brain activations during facial emotion recognition in humans. Neurosci Lett, 342(1-2), 101-104.

Streit, M., Ioannides, A. A., Liu, L., Wolwer, W., Dammers, J., Gross, J., et al. (1999). Neurophysiological correlates of the recognition of facial expressions of emotion as revealed by magnetoencephalography. Brain Res Cogn Brain Res, 7(4), 481-491.

Streit, M., Wolwer, W., Brinkmeyer, J., Ihl, R., \& Gaebel, W. (2000). Electrophysiological correlates of emotional and structural face processing in humans. Neurosci Lett, 278(1-2), 13-16.

Sutton, S. K., \& Davidson, R. J. (1997). Prefrontal brain asymmetry: a biological substrate of the behavioral approach and inhibition systems. Psychological Sciene, 8, 204210.

Sutton, S. K., Davidson, R. J., Donzella, B., Irwin, W., \& Dottl, D. A. (1997). Manipulating affective state using extended picture presentations. Psychophysiology, 34(2), 217-226.

Suzuki, A., Hoshino, T., Shigemasu, K., \& Kawamura, M. (2006). Disgust-specific impairment of facial expression recognition in Parkinson's disease. Brain, 129(Pt 3), 707-717.

Talairach, J., \& Tournoux, P. (1988). Co-planar stereotaxic atlas of the human brain. New York: Thieme.

Theeuwes, J. (1995). Perceptual selectivity for color and form: On the nature of the interference effect. In A. F. Kramer, M. G. H. Coles \& G. D. Logan (Eds.), Converging operations in the study of visual selective attention (pp. 297-314). Washington, DC: APA.

Theeuwes, J. (2005). Irrelevant singletons capture attention. In L. Itti, G. Rees \& J. Tsotsos (Eds.), Neurobiology of attention. San Diego, CA: Elsevier. 
Thomas, K. M., Drevets, W. C., Whalen, P. J., Eccard, C. H., Dahl, R. E., Ryan, N. D., et al. (2001). Amygdala response to facial expressions in children and adults. Biol Psychiatry, 49(4), 309-316.

Thulborn, K. R., Waterton, J. C., Matthews, P. M., \& Radda, G. K. (1982). Oxygenation dependence of the transverse relaxation time of water protons in whole blood at high field. Biochim Biophys Acta, 714(2), 265-270.

Tomita, H., Ohbayashi, M., Nakahara, K., Hasegawa, I., \& Miyashita, Y. (1999). Topdown signal from prefrontal cortex in executive control of memory retrieval. Nature, 401(6754), 699-703.

Tong, F. (2003). Primary visual cortex and visual awareness. Nat Rev Neurosci, 4(3), 219229.

Tootell, R. B., Dale, A. M., Sereno, M. I., \& Malach, R. (1996). New images from human visual cortex. Trends Neurosci, 19(11), 481-489.

Tootell, R. B., Hadjikhani, N., Hall, E. K., Marrett, S., Vanduffel, W., Vaughan, J. T., et al. (1998). The retinotopy of visual spatial attention. Neuron, 21(6), 1409-1422.

Tootell, R. B., Reppas, J. B., Dale, A. M., Look, R. B., Sereno, M. I., Malach, R., et al. (1995a). Visual motion aftereffect in human cortical area MT revealed by functional magnetic resonance imaging. Nature, 375(6527), 139-141.

Tootell, R. B., Reppas, J. B., Kwong, K. K., Malach, R., Born, R. T., Brady, T. J., et al. (1995b). Functional analysis of human MT and related visual cortical areas using magnetic resonance imaging. J Neurosci, 15(4), 3215-3230.

Treisman, A. (1960). Contextual cues in selective listening. Quaterly Journal of Experimental Psychology, 12, 242-248.

Treisman, A. (1969). Strategies and models of selective attention. Psychological Review, 76, 282-299.

Treisman, A. (1988). Features and objects: The fourteenth Bartlett memorial lecture. Quaterly Journal of Experimental Psychology, 40A, 201-237.

Treisman, A., \& Gormican, S. (1988). Feature analysis in early vision: evidence from search asymmetries. Psychol Rev, 95(1), 15-48.

Treisman, A. M., \& Gelade, G. (1980). A feature-integration theory of attention. Cognit Psychol, 12(1), 97-136.

Treue, S., \& Maunsell, J. H. (1996). Attentional modulation of visual motion processing in cortical areas MT and MST. Nature, 382(6591), 539-541. 
Tsal, Y. (1983). Movements of attention across the visual field. J Exp Psychol Hum Percept Perform, 9(4), 523-530.

Van der Heijden, A. H. C., Wolters, G., Groep, J. C., \& Hagenaar, R. (1987). Single-letter recognition accuracy benefits from advance cuing of location. Perception and Psychophysics, 42, 503-509.

Van Essen, D. (1995). Behind the optic nerve: an inside view of the primate visual system. Trans Am Ophthalmol Soc, 93, 123-133.

Van Essen, D. (2005). Corticocortical and thalamocortical information flow in the primate visual system. Prog Brain Res., 149, 173-185.

Van Essen, D. C., Newsome, W. T., \& Maunsell, J. H. (1984). The visual field representation in striate cortex of the macaque monkey: asymmetries, anisotropies, and individual variability. Vision Res, 24(5), 429-448.

Van Voorhis, S. T., \& Hillyard, S. A. (1977). Visual evoked potentials and selective attention to points in space. Perception and Psychophysics, 22, 54-62.

Villringer, A., \& Dirnagl, U. (1995). Coupling of brain activity and cerebral blood flow: basis of functional neuroimaging. Cerebrovasc Brain Metab Rev, 7(3), 240-276.

Vuilleumier, P., Armony, J. L., Clarke, K., Husain, M., Driver, J., \& Dolan, R. J. (2002). Neural response to emotional faces with and without awareness: event-related fMRI in a parietal patient with visual extinction and spatial neglect. Neuropsychologia, 40(12), 2156-2166.

Vuilleumier, P., Armony, J. L., Driver, J., \& Dolan, R. J. (2001). Effects of attention and emotion on face processing in the human brain: an event-related fMRI study. Neuron, 30(3), 829-841.

Vuilleumier, P., Armony, J. L., Driver, J., \& Dolan, R. J. (2003). Distinct spatial frequency sensitivities for processing faces and emotional expressions. Nat Neurosci, 6(6), 624-631.

Vuilleumier, P., \& Driver, J. (2007). Modulation of visual processing by attention and emotion: windows on causal interactions between human brain regions. Philos Trans R Soc Lond B Biol Sci, 362(1481), 837-855.

Vuilleumier, P., Richardson, M. P., Armony, J. L., Driver, J., \& Dolan, R. J. (2004). Distant influences of amygdala lesion on visual cortical activation during emotional face processing. Nat Neurosci, 7(11), 1271-1278. 
Wager, T. D., Phan, K. L., Liberzon, I., \& Taylor, S. F. (2003). Valence, gender, and lateralization of functional brain anatomy in emotion: a meta-analysis of findings from neuroimaging. Neuroimage, 19(3), 513-531.

Waldvogel, D., van Gelderen, P., Immisch, I., Pfeiffer, C., \& Hallett, M. (2000). The variability of serial fMRI data: correlation between a visual and a motor task. Neuroreport, 11(17), 3843-3847.

Wandell, B. A. (1999). Computational neuroimaging of human visual cortex. Annu Rev Neurosci, 22, 145-173.

Wandell, B. A., Brewer, A. A., \& Dougherty, R. F. (2005). Visual field map clusters in human cortex. Philos Trans R Soc Lond B Biol Sci, 360(1456), 693-707.

Watanabe, T., Sasaki, Y., Miyauchi, S., Putz, B., Fujimaki, N., Nielsen, M., et al. (1998). Attention-regulated activity in human primary visual cortex. J Neurophysiol, 79(4), 2218-2221.

Watson, D., Clark, L. A., \& Tellegen, A. (1988). Development and validation of brief measures of positive and negative affect: the PANAS scales. J Pers Soc Psychol, 54(6), 1063-1070.

Watson, D., \& Tellegen, A. (1985). Toward a consensual structure of mood. Psychol Bull, 98(2), 219-235.

Watson, J. (1919). A schematic outline of the emotions. Psychological Review, 26, 165196.

Weichselgartner, E., \& Sperling, G. (1987). Dynamics of automatic and controlled visual attention. Science, 238(4828), 778-780.

Weymouth, F. W. (1958). Visual sensory units and the minimal angle of resolution. Am J Ophthalmol, 46(1, Part 2), 102-113.

Whalen, P. J., Bush, G., McNally, R. J., Wilhelm, S., McInerney, S. C., Jenike, M. A., et al. (1998). The emotional counting Stroop paradigm: a functional magnetic resonance imaging probe of the anterior cingulate affective division. Biol Psychiatry, 44(12), 1219-1228.

Whalen, P. J., Rauch, S. L., Etcoff, N. L., McInerney, S. C., Lee, M. B., \& Jenike, M. A. (1998). Masked presentations of emotional facial expressions modulate amygdala activity without explicit knowledge. $J$ Neurosci, 18(1), 411-418.

Wild, B., Erb, M., \& Bartels, M. (2001). Are emotions contagious? Evoked emotions while viewing emotionally expressive faces: quality, quantity, time course and gender differences. Psychiatry Res, 102(2), 109-124. 
Williams, M. A., McGlone, F., Abbott, D. F., \& Mattingley, J. B. (2005). Differential amygdala responses to happy and fearful facial expressions depend on selective attention. Neuroimage, 24(2), 417-425.

Williams, M. A., Morris, A. P., McGlone, F., Abbott, D. F., \& Mattingley, J. B. (2004). Amygdala responses to fearful and happy facial expressions under conditions of binocular suppression. J Neurosci, 24(12), 2898-2904.

Winston, J. S., Strange, B. A., O'Doherty, J., \& Dolan, R. J. (2002). Automatic and intentional brain responses during evaluation of trustworthiness of faces. Nat Neurosci, 5(3), 277-283.

Winston, J. S., Vuilleumier, P., \& Dolan, R. J. (2003). Effects of low-spatial frequency components of fearful faces on fusiform cortex activity. Curr Biol, 13(20), 18241829.

Wojciulik, E., Kanwisher, N., \& Driver, J. (1998). Covert visual attention modulates facespecific activity in the human fusiform gyrus: fMRI study. J Neurophysiol, 79(3), 1574-1578.

Womelsdorf, T., Anton-Erxleben, K., Pieper, F., \& Treue, S. (2006). Dynamic shifts of visual receptive fields in cortical area MT by spatial attention. Nat Neurosci, 9(9), 1156-1160.

Wright, C. I., Fischer, H., Whalen, P. J., McInerney, S. C., Shin, L. M., \& Rauch, S. L. (2001). Differential prefrontal cortex and amygdala habituation to repeatedly presented emotional stimuli. Neuroreport, 12(2), 379-383.

Wright, C. I., Martis, B., Shin, L. M., Fischer, H., \& Rauch, S. L. (2002). Enhanced amygdala responses to emotional versus neutral schematic facial expressions. Neuroreport, 13(6), 785-790.

Wright, P., \& Liu, Y. (2006). Neutral faces activate the amygdala during identity matching. Neuroimage, 29(2), 628-636.

Wundt, W. (1897). Outlines of Psychology. Leipzig: Engelmann.

Wurtz, R. H., \& Mohler, C. W. (1976). Organization of monkey superior colliculus: enhanced visual response of superficial layer cells. J Neurophysiol, 39(4), 745-765.

Yamasaki, H., LaBar, K. S., \& McCarthy, G. (2002). Dissociable prefrontal brain systems for attention and emotion. Proc Natl Acad Sci U S A, 99(17), 11447-11451.

Yang, T. T., Menon, V., Eliez, S., Blasey, C., C.D., W., Reid, A. J., et al. (2002). Amygdalar activation associated with positive and negative facial expressions. Neuroreport, 13(14), 1737-1741. 
Yantis, S. (1993). Stimulus-driven attentional capture and attentional control settings. $J$ Exp Psychol Hum Percept Perform, 19(3), 676-681.

Yantis, S. (1995). Attentional capture in vision. In A. F. Kramer, M. G. H. Coles \& G. D. Logan (Eds.), Converging operations in the study of visual selective attention (pp. 45-76). Washington, DC: APA.

Yantis, S. (1998). Control of visual attention. In H. E. Pashler (Ed.), Attention (pp. 223256). Hove, England: Psychology Press/Erlbaum.

Yantis, S., \& Johnston, J. C. (1990). On the locus of visual selection: evidence from focused attention tasks. J Exp Psychol Hum Percept Perform, 16(1), 135-149.

Yantis, S., \& Jonides, J. (1990). Abrupt visual onsets and selective attention: voluntary versus automatic allocation. J Exp Psychol Hum Percept Perform, 16(1), 121-134.

Yeshurun, Y., \& Carrasco, M. (1998). Attention improves or impairs visual performance by enhancing spatial resolution. Nature, 396(6706), 72-75.

Young, A. W., Aggleton, J. P., Hellawell, D. J., Johnson, M., Broks, P., \& Hanley, J. R. (1995). Face processing impairments after amygdalotomy. Brain, 118 ( Pt 1), 1524.

Yucel, G., McCarthy, G., \& Belger, A. (2007). fMRI reveals that involuntary visual deviance processing is resource limited. Neuroimage, 34(3), 1245-1252.

Zald, D. H. (2003). The human amygdala and the emotional evaluation of sensory stimuli. Brain Res Brain Res Rev, 41(1), 88-123.

Zald, D. H., Lee, J. T., Fluegel, K. W., \& Pardo, J. V. (1998). Aversive gustatory stimulation activates limbic circuits in humans. Brain, 121 ( Pt 6), 1143-1154. 


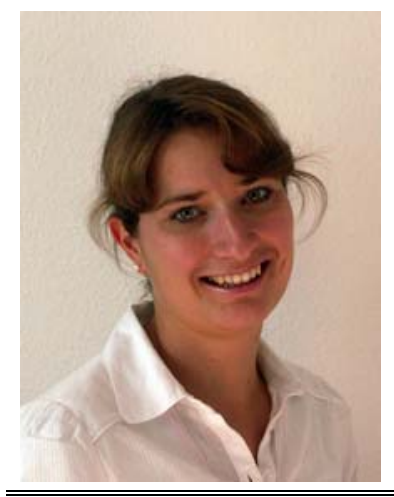

\section{Mag. Carmen Morawetz}

Christophorusweg 8

37075 Göttingen

Tel.: 0551/9953399

Mobile: 0176/23509123

Email: cmorawe@gwdg.de

Date of Birth

Nationality

\section{Education}

since 10/04

09/03-04/04

$1999-2004$

$2001-2002$
25.10.1980

Austria

\section{PhD Student in Psychology}

Georg-August-University Göttingen

Cognitive Neuroscience Laboratory; German Primate Center

Supervisor: Prof. Dr. Stefan Treue

MR-Research in Neurology and Psychiatry;

Supervisor: Dr. Peter Dechent

MSc (Mag.rer.nat.) in Psychology

University of Klagenfurt

Master Thesis: "Emotional content of visual stimuli influences memory retrieval: an fMRI study"

Dept. of Psychiatry and Psychotherapy I, Laboratory for Neurophysiology and Neuroimaging and Dept. of Psychosomatic Medicine and Psychotherapy, Johann Wolfgang Goethe University Frankfurt; Supervisor: PD Dr. David E. J. Linden

Dept. of Psychology, Cognitive Psychology Unit, University of Klagenfurt; Supervisor: Prof. Dr. Oliver Vitouch

\section{Student of Psychology}

University of Klagenfurt

Student of Cognitive Neuroscience and Psychology

University of Bremen 


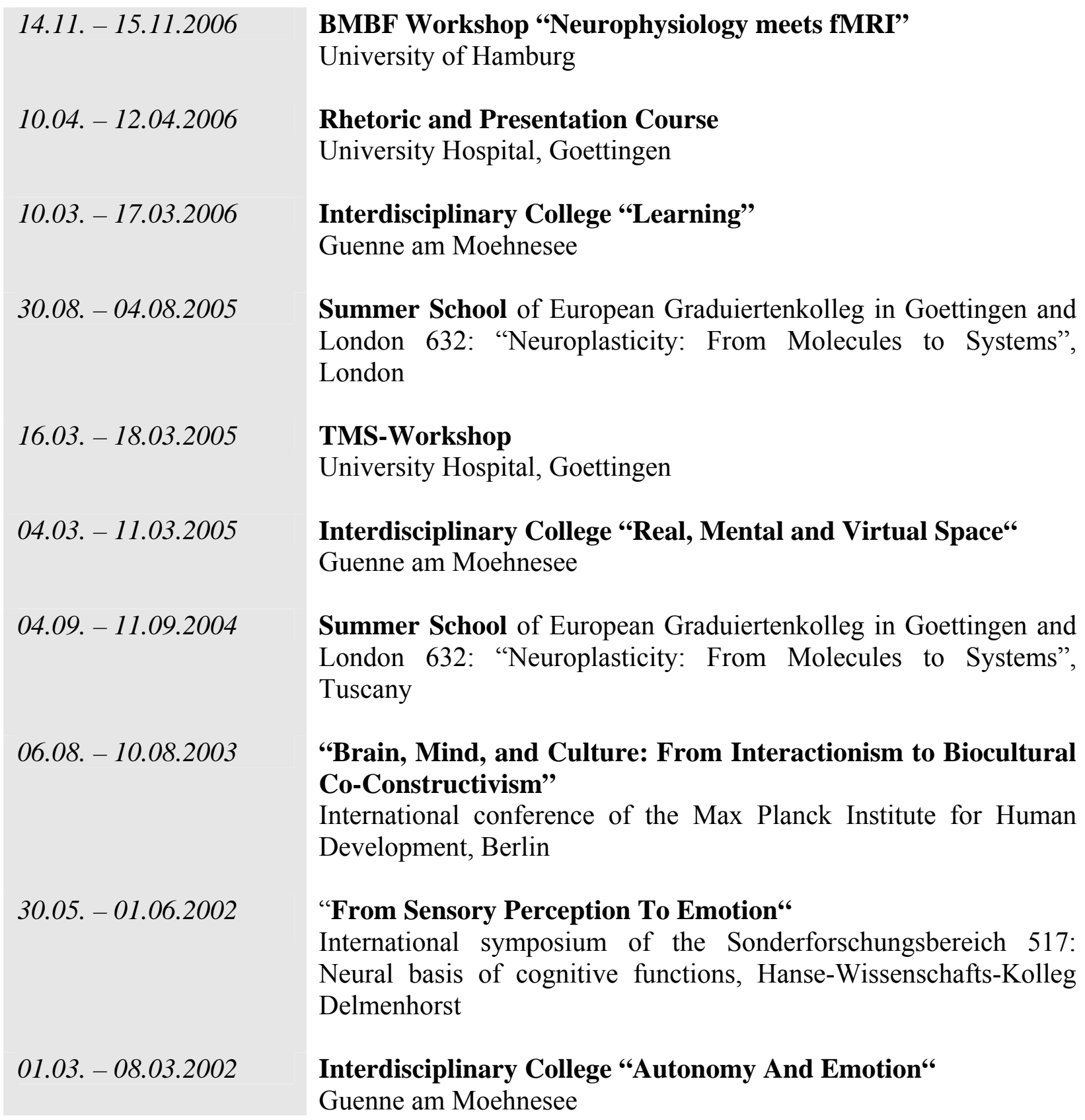




\begin{tabular}{|c|c|}
\hline 03.03. - 30.06.2003 & $\begin{array}{l}\text { Teaching Assistant at the Cognitive Psychology Unit, Department of } \\
\text { Psychology } \\
\text { University of Klagenfurt }\end{array}$ \\
\hline 03.02. - 28.02.2003 & $\begin{array}{l}\text { Dept. of Psychiatry and Psychotherapy I, Laboratory for } \\
\text { Neurophysiology and Neuroimaging and Dept. of Psychosomatic } \\
\text { Medicine and Psychotherapy } \\
\text { Johann Wolfgang Goethe University Frankfurt, Frankfurt am Main }\end{array}$ \\
\hline 15.04. - 15.07.2002 & $\begin{array}{l}\text { Teaching Assistant at the Department of Human-Neurobiology } \\
\text { University of Bremen }\end{array}$ \\
\hline 01.04. - 05.07.2002 & $\begin{array}{l}\text { Institute for Brain Research } \\
\text { University of Bremen }\end{array}$ \\
\hline 09.01. - 30.04.2002 & $\begin{array}{l}\text { Department of Neuropsychology \& Behavioral Neurobiology } \\
\text { University of Bremen }\end{array}$ \\
\hline 16.07. - 17.08.2001 & $\begin{array}{l}\text { Arbeitsvereinigung der Sozialhilfe Kärntens } \\
\text { Klagenfurt }\end{array}$ \\
\hline
\end{tabular}

\section{$\underline{\text { Journal publications }}$}

Morawetz, C., Holz, P., Baudewig, J., Treue, S., Dechent, P. (2007). Split of attentional resources in human visual cortex. Visual Neuroscience, 24(6): 817-26.

Morawetz, C., Holz, P., Lange, C., Baudewig, J., Weniger, G., Irle, E., Dechent, P. (2008). Improved functional mapping of the human amygdala using a standard functional magnetic resonance omaging sequence with simple modifications. Magnetic Resonance Imaging, 26(1): 45-53.

Von Steinbuechel, N., Richter, S., Morawetz, C., Riemsma, R. (2005). Assessment of subjective health and health-related quality of life in persons with acquired or degenerative brain injury. Current Opinion in Neurology, 18(6): 681-91.

\section{$\underline{\text { Published abstracts }}$}

Morawetz C, Kaping D, Baudewig J, Treue S, Webster M, Dechent P (2007). Neural mechanisms underlying the face distortion aftereffect. Poster presented at the 13th Annual Meeting of the organization of Human Brain Mapping, Chicago, Illinois, USA.

Morawetz C, Holz P, Baudewig J, Treue S, Dechent P (2006). Multiple spotlights of visual attention? Poster presented at the 5 th Forum of European Neuroscience, Vienna, Austria.

Morawetz C, Holz P, Baudewig J, Treue S, Dechent P (2006). Multiple spotlights of visual attention? Poster presented at the 12th Annual Meeting of the organization of Human Brain Mapping, Florence, Italy. 
Morawetz C, Lange C, Holz P, Baudewig J, Weniger G, Irle E, Dechent P (2006). Functional MRI of the human amygdala avoiding susceptibility artefacts. Poster presented at the 12th Annual Meeting of the organization of Human Brain Mapping, Florence, Italy.

Morawetz C, Holz P, Baudewig J, Treue S, Dechent P (2006). Multiple spotlights of visual attention? Talk presented at the ISMRM 14th Annual Meeting, Seattle, USA.

Morawetz C, Lange C, Holz P, Baudewig J, Weniger G, Irle E, Dechent P (2006). Functional MRI of the human amygdala avoiding susceptibility artefacts. Poster presented at the ISMRM 14th Annual Meeting, Seattle, USA.

Morawetz C, van de Ven VG, Linden DEJ, Vitouch O, \& Roeder CH (2004). Emotional content of visual stimuli influences encoding activation: An fMRI study. Poster presented at the 10th Annual Meeting of the Organization for Human Brain Mapping, Budapest, Hungary.

Göttingen, am 27. Mai 2008 